\title{
On Minmax Robustness for Multiobjective Optimization with Decision or Parameter Uncertainty
}

\section{DissERTATION}

zur Erlangung des mathematisch-naturwissenschaftlichen Doktorgrades

\author{
"Doctor rerum naturalium" \\ der Georg-August-Universität Göttingen \\ im Promotionsstudiengang Mathematical Sciences \\ der Georg-August University School of Science (GAUSS)
}

vorgelegt von

Corinna KrüGer

aus Gifhorn

Göttingen, 2018 



\section{Betreuungsausschuss:}

Prof. Dr. Anita Schöbel

Institut für Numerische und Angewandte Mathematik

Georg-August-Universität Göttingen

Prof. Dr. Russell Luke

Institut für Numerische und Angewandte Mathematik

Georg-August-Universität Göttingen

Mitglieder der Prüfungskommission:

\section{Referentin:}

Prof. Dr. Anita Schöbel

Institut für Numerische und Angewandte Mathematik

Georg-August-Universität Göttingen

\section{Korreferentin:}

Prof. Dr. Gabriele Eichfelder

Institut für Mathematik

Technische Universität Ilmenau

\section{Weitere Mitglieder der Prüfungskommission:}

Prof. Dr. Jörg Brüdern

Mathematisches Institut

Georg-August-Universität Göttingen

JProf. Dr. Anja Fischer

Fakultät Wirtschaftswissenschaften

Technische Universität Dortmund

Prof. Dr. Stephan Huckemann

Institut für Mathematische Stochastik

Georg-August-Universität Göttingen

Prof. Dr. Gert Lube

Institut für Numerische und Angewandte Mathematik Georg-August-Universität Göttingen

Tag der mündlichen Prüfung: 29. März 2018 



\section{Contents}

1. Introduction 1

2. Literature Review 3

2.1. Robust Single-Objective Optimization . . . . . . . . . . . . . 3

2.2. Robust Multiobjective Optimization . . . . . . . . . . . . . . . . 4

3. Preliminaries and Notation $\quad 11$

3.1. Minmax Robustness for Uncertain Single-Objective Optimization . . . . 11

3.2. Deterministic Multiobjective Optimization . . . . . . . . . . . . . 14

3.3. Set-Valued Optimization . . . . . . . . . . . . . . 16

3.4. Minmax Robustness for Parameter Uncertainty in MOPs . . . . . . . . . 17

3.4.1. Point-Based Robust Efficiency . . . . . . . . . . . . . . . . . . 18

3.4.2. Set-Based Robust Efficiency . . . . . . . . . . . . . . . . . 18

3.4.3. Relation between the two Concepts . . . . . . . . . . . . . . . . . 19

4. Summary of the publications $\quad 21$

4.1. Decision Uncertainty in Multiobjective Optimization . . . . . . . . . 22

4.2. The Robustness Gap for Uncertain Multiobjective Optimization . . . . . 27

4.3. An Application of Robust Multiobjective Optimization in Agriculture . . 34

4.3.1. Peat and Pots: An Application . . . . . . . . . . . . . . 34

4.3.2. Peat and Pots: Analysis of Robust Solutions . . . . . . . . . . . . 40

$\begin{array}{ll}\text { 5. Discussion } & 47\end{array}$

$\begin{array}{ll}\text { 6. Conclusions } & 51\end{array}$

$\begin{array}{ll}\text { Bibliography } & 53\end{array}$

$\begin{array}{ll}\text { Addenda } & 61\end{array}$

$\begin{array}{ll}\text { A. Decision Uncertainty in Multiobjective Optimization } & 63\end{array}$

B. The Robustness Gap for Uncertain Multiobjective Optimization 97

C. Peat and Pots: An Application of Robust Multiobjective Optimization to $\begin{array}{ll}\text { a Mixing Problem in Agriculture } & 131\end{array}$

D. Peat and pots: Analysis of Robust Solutions for a Biobjective Problem in Agriculture 



\section{Introduction}

Decision problems occur in various forms and under various circumstances in our casual and professional life. Sometimes, there is a unique goal that we want to optimize, but mostly we want to achieve several goals simultaneously. Moreover, we often lack complete knowledge of the decision problem's data or we suspect that we might not be able to put a selected solution into practice exactly as intended.

Decision problems of this kind are investigated in this thesis and they are called uncertain multiobjective optimization problems. A problem is called multiobjective (MOP) if it has two or more objective functions that are in conflict with each other, which means that optimizing all objective functions simultaneously is impossible. When considering uncertain optimization problems, the two types of uncertainty parameter uncertainty and decision uncertainty are typically distinguished. Parameter uncertainty means that the problem parameters are not known precisely at the time when the problem is solved and decision uncertainty means that the decision variables of the problem cannot be implemented exactly as targeted. It is generally assumed that uncertain parameters or decision variables vary within a known set, which is called the uncertainty set, and the elements of this set are called scenarios. In case of decision uncertainty, the uncertainty set is also called perturbation set. A multiobjective uncertain problem is therefore a multiobjective problem whose parameters or decision variables are affected by uncertainty.

The following example for such a decision problem is also studied in detail later in this thesis. For the commercial cultivation of potted plants, a plant nursery aims to choose the planter pots and the growing substrate such that an economic and an environmental objective function are minimized. The economic function is the difference of the costs and the selling price and the environmental objective function is the global warming potential. While it is common to consider an economic objective, the environmental objective is motivated by recent research on the negative environmental impacts of the currently used pots and growing substrate. In this problem, the parameters are uncertain due to annual fluctuations in the costs of the input materials and due to imprecisions in the measurement of the global warming potential. Decision uncertainty is also present, because the growing substrate can be any mixture of peat and compost and the mixing is only performed roughly, which leads to different mixtures in different pots.

Uncertain multiobjective optimization is hence of practical interest. However, a solution concept is required in order to solve uncertain MOPs. A research area that has recently been developed to address this kind of problem is robust multiobjective optimization. Various concepts of robustness in the literature define which solutions are considered robust feasible or robust efficient for uncertain MOPs, where efficiency is the multiobjective equivalent of optimality. A robustness concept is generally associated with a so-called robust counterpart, which is a deterministic problem whose solutions are 
defined to be the robust solutions to the uncertain problem.

One of the well-known and most studied robustness concepts is minmax robustness. Minmax robustness was originally developed for uncertain single-objective optimization and it has been studied for decades, see Ben-Tal, Ghaoui, and Nemirovski 2009. According to this robustness concept, solutions are considered robust feasible if they are feasible for all scenarios, and robust optimal if they are the best solutions with respect to the worst-case scenarios. Based on single-objective minmax robustness, various multiobjective minmax robustness concepts have been proposed in the literature and all have in common that robust efficient solutions are defined to be those that are best in the worst-case scenarios. In this thesis, two frequently studied minmax robustness concepts for MOPs with parameter uncertainty are considered and a minmax robustness concept for MOPs with decision uncertainty is introduced and investigated in the first publication contributing to this thesis.

Minmax robustness is by definition a conservative approach to uncertainty, because solutions are compared with respect to the worst-case scenario only. Conversely, an optimistic approach is to choose some scenario and to determine its optimal solutions. To measure the gap between this optimistic approach and the conservative minmax robustness approach, the robustness gap has been defined in single-objective optimization. So far, no concept of a multiobjective robustness gap has been proposed. In the second publication of this thesis, a multiobjective robustness gap is introduced and lower and upper bounds for the gap are studied.

In the third and fourth publication of this thesis, the multiobjective minmax robustness concept from the first publication is applied to determine the robust efficient solutions for a case study of the motivating example in this introduction.

The thesis is structured as follows: In Chapter 2, a literature review on minmax robustness for single-objective optimization, minmax robustness for multiobjective optimization and on further robustness concepts for uncertain MOPs is given. In Chapter 3, the notation of this thesis is introduced and known definitions and results from the literature that are used subsequently are recalled. In Chapter 4, the four publications contributing to this thesis are summarized. In Chapter 5, the contributions of the four publications to the topic of this thesis and the connections between the individual publications are discussed. In Chapter 6 , the results of this thesis are summarized and options of further research about minmax robustness for uncertain multiobjective optimization are given. 


\section{Literature Review}

This thesis contributes to the young field of robust multiobjective optimization, which is one of the research areas that address multiobjective optimization problems (MOPs) that are affected by uncertainty. The three main areas dealing with uncertain MOPs are fuzzy optimization, stochastic optimization and robust optimization. The former two approaches are widely studied in the literature, see, e.g., Caballero, Cerdá, Muñoz, et al. 2001; Zimmermann 2012 for surveys, and see Huang and Teghem 2012 for an early comparison of these two classical approaches. However, in fuzzy and stochastic optimization, it is required that some distribution function on the uncertainty set is known. The approach robust multiobjective optimization has been developed to handle uncertain problems where only the uncertainty set is known and where information about any distribution on the uncertainty set is missing. It has mainly been researched in recent years, but robust single-objective optimization has been studied for decades.

\subsection{Robust Single-Objective Optimization}

Robust optimization was initially developed and extensively studied for uncertain singleobjective problems and many robustness concepts, i.e., ideas of what is considered to be a robust solution, have been proposed and studied in the literature. One of the well-known and most studied single-objective robustness concepts is minmax robustness, see, e.g., Ben-Tal, Ghaoui, and Nemirovski 2009 for a comprehensive textbook and see Soyster 1973 for an early study thereof. Minmax robust solutions are defined as those that are feasible for all scenarios and that perform best in the worst-case scenarios.

In the minmax robust optimization literature, parameter uncertainty and decision uncertainty are typically distinguished, but each of the two is addressed under various names. Parameter uncertainty is also referred to as uncertain data, see, e.g., Ben-Tal, Ghaoui, and Nemirovski 2009; Ben-Tal and Nemirovski 1998, and studies of decision uncertainty are also frequently found under the name implementation error, see Ben-Tal and Hertog 2011; Ben-Tal and Nemirovski 2002; Bertsimas and Nohadani 2010; Bertsimas, Nohadani, and Teo 2007, 2010b; Stinstra and Hertog 2008 or robust regularization, see Bayer and Sendhoff 2007; Lewis and Pang 2009 or under the general name robustness, see Das 1997.

This thesis contributes to the investigation of minmax robustness for multiobjective optimization with decision and/or parameter uncertainty. A minmax robustness concept for MOPs with decision uncertainty is proposed and analyzed in the first publication contributing to this thesis. Moreover, this minmax robustness concept is combined with a well-known minmax robustness concept for parameter uncertainty in MOPs and it is 
applied in a case study to determine robust solutions to a biobjective problem with decision and parameter uncertainty. This application is described in the third publication contributing to this thesis.

Minmax robustness is a conservative concept. Conversely, an optimistic approach would be to chose a solution that is optimal for some scenario and to hope for that scenario. In order to compare minmax robust solutions to the optimal solutions of the scenarios the concept of the robustness gap has been introduced in Ben-Tal and Nemirovski 1998, 1999. The robustness gap measures the distance between the robust optimal objective value and the supremum of the optimal objective values of the scenarios. Therefore, studies of minmax robustness naturally lead to investigations of the robustness gap.

A novel concept for a multiobjective robustness gap is proposed and investigated in the second publication contributing to this thesis. To the best knowledge of the author of this thesis, it is the first approach to define a multiobjective robustness gap and the proposed gap is applicable to one of the most studied concepts among the various minmax robustness concepts for MOPs in the literature.

For surveys and/or comparisons of further single-objective robustness concepts that have been proposed in the literature see, e.g., Bayer and Sendhoff 2007; Bertsimas, Brown, and Caramanis 2011; Bertsimas and Sim 2004; Gabrel, Murat, and Thiele 2014; Goerigk and Schöbel 2016; Klamroth, Köbis, Schöbel, and Tammer 2017.

\subsection{Robust Multiobjective Optimization}

The topic of this thesis is minmax robustness for MOPs with decision and/or parameter uncertainty. Based on single-objective minmax robustness, multiobjective robustness concepts are considered minmax robustness concepts here, if a solution is robust feasible whenever it is feasible for all scenarios and if a solution is robust efficient whenever it is efficient with respect to an objective function representing the worst cases of the original objective function over the scenarios. In contrast to single-objective optimization, the notion of a worst-case is not clear in multiobjective optimization. Therefore, there are various options of how to define multiobjective minmax robustness.

In the robust multiobjective optimization literature, decision and parameter uncertainty are typically addressed with distinct robustness concepts in distinct papers. Decision uncertainty in MOPs is scarcely treated in the literature and an overview on robustness concepts for this topic is provided next. The major part of the robust multiobjective optimization literature is concerned with parameter uncertainty and an overview of minmax robustness for parameter uncertainty is given. Also, a summary of further multiobjective robustness concepts is stated to reflect the variety of concepts in the literature. 


\section{Robustness for MOPs with Decision Uncertainty}

Among the multiobjective robustness concepts in the literature, those that are developed to address decision uncertainty in MOPs are of particular interest in this thesis, because a minmax robustness concept for MOPs with decision uncertainty is defined and analyzed in the first publication contributing to this thesis.

One of the earliest multiobjective robustness concept proposed in the literature addresses decision uncertainty. Deb and Gupta 2005, 2006 propose two different robustness concepts for MOPs with decision uncertainty and they claim that theirs is the first work on robust multiobjective optimization. The authors first define a new deterministic MOP by replacing every component of the objective function by the mean value of the objective in a given neighborhood of the solution. A Multi-objective Robust Solution of Type 1 is then defined as an efficient solution to the latter problem. As a second robustness concept, the authors add a new constraint to the MOP, allowing only solutions whose outcome vector is within a fixed distance from the previously used mean objective function, and an efficient solution to the newly generated MOP is denoted as a Multi-objective Robust Solution of Type 2.

Around the same time, Barrico and Antunes 2006 suggest a concept of sensitivity analysis for MOPs with decision uncertainty, called the degree of robustness, and for each solution the size of its neighborhood is scaled such that the outcome of the solution and the outcomes of its neighbors are within a given distance.

Neither of these approaches is based on evaluating worst case scenarios and hence, neither is a minmax robustness approach. A minmax robustness approach for decision uncertainty in MOPs with finitely many feasible solutions and finitely many realizations is applied in Avigad and Branke 2008. Though that paper mainly addresses parameter uncertainty in MOPs, also for each solution a finite set of realizations is considered. An evolutionary algorithm is then applied in two steps. In the first step, for each solution a set of worst case scenarios is searched and in the second step, each pair of solutions and the images of their respective worst case scenarios are compared in a point-wise way.

In the first publication contributing to this thesis, the robustness concept decision robust efficiency for decision uncertainty in MOPs is introduced and analyzed. Among other results, it is shown that the concept corresponds to comparing solutions by their worst case outcomes that form specific sets in the objective space, known as supremal sets. Therefore, the proposed concept is considered a minmax robustness concept for decision uncertainty in MOPs.

\section{Minmax Robustness for MOPs with Parameter Uncertainty}

Parameter uncertainty in MOPs is recently addressed by different minmax robustness concepts and two of the most frequently studied minmax robustness concepts in the literature are of particular interest for this thesis. To distinguish the two concepts, the names point-based robust efficiency and set-based robust efficiency are used subsequently, since both concepts are called robust efficiency in the respective original papers. 
Point-based robust efficiency goes back to Kuroiwa and Lee 2012. A new deterministic problem, the so-called robust counterpart, is defined by replacing every component of the objective function with its supremum over the uncertainty set. Point-based robust efficient solutions are then defined as the efficient solutions to the robust counterpart, which is an MOP. The robust counterpart can equivalently be defined by replacing the objectives of the MOP with their suprema and transferring them to the constraints in the way proposed by Ben-Tal, Ghaoui, and Nemirovski 2009 for single-objective minmax robustness. Point-based robust efficiency has frequently been studied, see, e.g., Chuong 2016; Doolittle, Kerivin, and Wiecek 2015; Fakhar, Mahyarinia, and Zafarani 2017; Fliege and Werner 2014; Goberna, Jeyakumar, Li, and Vicente-Pérez 2014, 2015; Kim 2013a,b; Lee and Lee 2016; Wang, Liu, and Chai 2015.

This minmax robustness concept is considered in the second, third and fourth publications of this thesis. In the second publication, a multiobjective robustness gap is defined with respect to this robustness concept.

Set-based robust efficiency is predicated on the idea to compare solutions by their whole outcome sets, i.e., the set of objective function vectors for all scenarios. The robust counterpart of this robustness concept is a set-valued optimization problem, and set dominance is defined through the order induced by the Pareto cone. The concept is introduced in Ehrgott, Ide, and Schöbel 2014 and it is extended to various order relations for set-valued problems in Bischoff, Jahn, and Köbis 2017; Ide 2014; Ide and Köbis 2014; Ide, Köbis, Kuroiwa, et al. 2014. Comparing the outcomes for all scenarios can be considered a minmax approach, because it is equivalent to comparing the outcomes of certain worst-case scenarios that are obtained by solving a second level MOP as done in Avigad and Branke 2008 with multiobjective evolutionary algorithms. Also, solutions are compared by their whole set of outcomes in different ways in Crespo, Bergez, and Garcia 2010. Scalarizations of uncertain MOPs are investigated as a method to determine setbased robust efficient solutions in Bokrantz and Fredriksson 2017; Wei, Chen, and Li 2017. In the former, also a similar set-valued robustness concept is proposed where solutions are compared by the convex hull of the outcomes of all scenarios rather than by the outcome set itself.

The concept of set-based robust efficiency is considered in the first, third and fourth publications contributing to this thesis. In the first publication, the introduced concept Decision robust efficiency for MOPs with decision uncertainty is based on the same idea as set-based robust efficiency, which is to compare solutions by the whole sets of possible realizations. In the third and fourth publication of this thesis, a special case of parameter uncertainty occurs in the presented application of multiobjective minmax robustness, and this specific uncertainty implies that point-based and set-based robust efficiency coincide. Therefore, the third and fourth publications can be considered as an application not only of decision robust efficiency, but also of point-based and of set-based robust efficiency.

The two multiobjective minmax robustness concepts point-based robust efficiency 
and set-based robust efficiency have various applications in the literature. Point-based robust efficiency has been applied to several fields, such as radiation therapy, see Chen, Unkelbach, Trofimov, et al. 2012, and game theory, see Yu and Liu 2013 and set-based robust efficiency has been applied to veneer production in Ide, Tiedemann, Westphal, and Haiduk 2015.

As an application of minmax robustness for uncertain MOPs, a mixing problem arising in agriculture is investigated in this thesis, and it is a biojective problem with one economic and one environmental objective function that is affected by decision and parameter uncertainty. It is noted that point-based and set-based robust efficiency have recently been applied to two case studies on similar problems, i.e., problems with economical and environmental objectives that are effected by parameter uncertainty. An application of point-based robust efficiency to supply chain planning for the German biodiesel market is given in Hombach, Büsing, and Walther 2017, and see Majewski, Wirtz, Lampe, and Bardow 2017 for an application of set-based robust efficiency to distributed energy supply system design. Note that the robust counterpart in the latter problem reduces to a deterministic MOP instead of a set-valued problem due to its specific uncertainty set and objective functions and hence, point-based and set-based minmax robust efficiency coincide in that publication.

In contrast, in the application presented in the third publication of this thesis, the set-valued minmax robust efficiency concept for decision uncertainty from the first publication is applied and minmax (decision) robust efficient solutions are obtained by solving a set-valued problem that cannot be reduced to a deterministic MOP.

For further multiobjective minmax robustness approaches in the literature, see, e.g., $\mathrm{Li}$ and Azarm 2008 where feasibility for all scenarios is required and, as a worst-case approach, the infinity-norm of all balls containing the outcome set is minimized. Feasibility for all scenarios is also required in Kalantari, Dong, and Davies 2016 and a deterministic $\mathrm{MOP}$ is generated by replacing the uncertain parameters in the objective function with their mean values, leading to another robustness concept that is related to minmax robustness by its concept of robust feasibility. Moreover, a robustness concept combining feasibility for all scenarios with minimizing the distance between the outcomes of the worst-case and best-case scenarios is studied in Besharati and Azarm 2006.

\section{Further Multiobjective Robustness Concepts}

Even though the research topic of robust multiobjective optimization has mainly been developed in recent years, it is noted that a specific class of uncertain MOPs has been studied already in the 1980s, namely linear MOPs with uncertain coefficients that lie in known intervals. Two solution concepts for these problems are suggested in Bitran 1980: solutions that are either efficient for all scenarios or for at least one scenario. However, the two solution concepts were not given names and they were not called robust approaches therein. The former is named necessarily efficient and the latter is named possibly efficient in Ida 1996 and for further research on this topic, see Hladík 2010, 2012; Ida 2003; Rivaz and Yaghoobi 2013 and see Oliveira and Antunes 2007 for a survey. The 
two solution concepts are later introduced as robustness concepts for general uncertain MOPs: necessary efficiency is also known as highly robust efficiency while possible efficiency is also called flimsily robust efficiency, see Ide and Schöbel 2016; Kuhn, Raith, Schmidt, and Schöbel 2016; Raith, Schmidt, Schöbel, and Thom 2017.

Apart from minmax robustness, there are various further multiobjective robustness concepts that are based on single-objective robustness concepts. Cardinality-constrained robustness, which is also referred to as budget of uncertainty, is applicable in the case that the uncertainty set consists of an interval for each parameter. It is assumed that only a predefined number of parameters can attain their worst-case values simultaneously. The concept is originally defined in Bertsimas and Sim 2004 for single-objective problems and it has been extended to MOPs by considering for each component of the objective function the worst case with respect to the cardinality-constrained uncertainty set, see, e.g., Hassanzadeh, Nemati, and Sun 2013, 2014; Raith, Schmidt, Schöbel, and Thom 2018; Wang, Li, Ding, et al. 2017. Note that this approach is similar to the extension of minmax robustness to point-based robust efficiency. In Schmidt, Schöbel, and Thom 2018, set-based robust efficient solutions to combinatorial MOPs with cardinalityconstrained uncertainty set are determined by means of scalarization techniques. Also, algorithms for finding robust efficient solutions to combinatorial MOPs with respect to cardinality-constrained, flimsily, highly, point-based and set-based robust efficiency are developed in Thom 2018.

Another well-known concept from single-objective optimization is regret robustness. Regret robust solutions are those that minimize the maximal regret which is defined for each solution and for each scenario as the difference between the objective function value of a solution and the optimal value of the scenario. The single-objective concept is studied in Kouvelis and $\mathrm{Yu} 1997$ and it is transferred to MOPs in various ways. In Xidonas, Mavrotas, Hassapis, and Zopounidis 2017, every component of the objective function is replaced by its maximal regret, which is again similar to the extension of minmax robustness to point-based robust efficiency. In Rivaz and Yaghoobi 2013, the maximum regret over all components of the objective function is minimized. In $\mathrm{Hu}$ and Mehrotra 2012, the regret of each component is weighted, thus leading to a scalarized objective function, and the maximum over a set of uncertain weights is minimized. In Nikulin, Karelkina, and Mäkelä 2013 a relative regret is minimized, which is itself calculated by maximizing the relative deviations of solutions at the smallest components of the objective function, i.e., a single-objective min-max-min problem is obtained.

Also, the single-objective robustness concept of light robustness, see, e.g., Fischetti and Monaci 2009; Schöbel 2014, has been extended to MOPs. Lightly robust feasible solutions are those that are feasible for a nominal scenario and whose objective value in the nominal scenario is within a fixed range of the optimal objective value. For all other scenarios, the distance to feasibility is measured and the solution that is closest to feasibility is considered lightly robust optimal. In the multiobjective case, see Ide and Schöbel 2016; Kuhn, Raith, Schmidt, and Schöbel 2016, a nominal scenario is selected 
and it is required that a solution is mapped by every component of the nominal objective function within a fixed range of its optimal value, which implies one constraint per component of the objective function. Lightly robust solutions are then considered as the solutions to the robust counterpart of set-based minmax efficiency that satisfy these additional constraints.

Some robustness concepts for uncertain MOPs are also related to sensitivity analysis. In this case, the uncertainty set is not fixed, but there is a basic set such as a norm ball whose size can be adjusted by multiplying each element with a nonnegative scalar. Instead of requiring that a certain condition is satisfied for the whole uncertainty set, each solution is mapped to the maximal size that the uncertainty set can have while the condition holds.

In Georgiev, Luc, and Pardalos 2013 a norm ball around the parameters of the objective function is considered and for each solution the maximum size of the norm ball such that the solution is efficient for all scenarios is called the radius of robustness. A solution is then considered robust if it has a positive radius of robustness and this robustness concept is also studied in Kabgani and Soleimani-damaneh 2018; Pourkarimi and Soleimani-damaneh 2016; Zamani, Soleimani-damaneh, and Kabgani 2015.

A related concept is the radius of robust feasibility as defined in Goberna, Jeyakumar, Li, and Vicente-Pérez 2014, 2015, which is defined as the maximum size of the uncertainty set under the condition that the MOP does still have a solution that is feasible for all scenarios. In Gunawan and Azarm 2005, the insensitivity of a solution for a fixed nominal scenario is defined as the maximum size of the uncertainty set such that for every scenario the objective vector of the solution is within a neighborhood of the objective vector of the nominal scenario.

Various further multiobjective robustness concepts have been proposed in the literature. One robustness concept is multi-scenario efficiency, where a new objective function is built by adding one deterministic objective function for each scenario and for each component of the uncertain objective function thereby creating a big objective function vector. The efficient solutions to the new MOP are then considered multi-scenario efficient, see, e.g., Botte and Schöbel 2016; Fadel, Haque, Blouin, and Wiecek 2005.

All scenarios in the uncertainty set are evaluated and compared by three different definitions of dominance in Crespo, Bergez, and Garcia 2010. Deterministic MOPs with uncertain decision maker preferences that are modeled by weighted-sum scalarization with uncertain weights are studied, e.g., in Farshidi, Rakai, Behjat, and Westwick 2016; $\mathrm{Hu}$ and Mehrotra 2012; Mavrotas, Pechak, Siskos, et al. 2015; Palma and Nelson 2010. Uncertain MOPs are scalarized and then investigated by means of single-objective robust optimization, e.g., in Asprion, Blagov, Böttcher, et al. 2017; Doolittle, Dranichak, Muir, and Wiecek 2016. Moreover, MOPs with one uncertain parameter are investigated in Witting 2012; Witting, Ober-Blöbaum, and Dellnitz 2013 with a variational approach where robust solutions are considered as paths in the decision space.

Note that uncertain MOPs have also been addressed frequently in the field of evolutionary optimization by considering a so-called robustness measure as an additional 
objective function. Many robustness measures have been proposed to suit specific problems as discussed in, e.g., Gabrel, Murat, and Thiele 2014; Gaspar-Cunha and Covas 2008 and the references therein.

For further multiobjective robustness concepts, see, e.g., the comprehensive surveys Ide and Schöbel 2016; Wiecek and Dranichak 2016 and the references therein. 


\section{Preliminaries and Notation}

In the following, the problems considered in this thesis are introduced and related definitions, results and solution concepts from the literature are recalled.

Throughout this thesis, for every set $S$, its interior, closure and boundary are denoted as int $(S), \operatorname{cl}(S)$ and bd $(S)$ respectively. For every $p \in \mathbb{N}$, the $p$-dimensional real vector space is denoted as $\mathbb{R}^{p}$.

\subsection{Minmax Robustness for Uncertain Single-Objective Optimization}

Two types of uncertainty, parameter uncertainty and decision uncertainty, are distinguished.

\section{Parameter Uncertainty}

For uncertain optimization with parameter uncertainty, each realization of the uncertain parameters is called a scenario and the set consisting of all scenarios is called the uncertainty set $\mathcal{U}$. In this thesis, it is assumed that the uncertainty set $\mathcal{U}$ is a compact subset of a finite dimensional vector space. A single-objective optimization problem with parameter uncertainty is given as

$$
\left\{\begin{aligned}
\left(\mathcal{P}^{s-o}(\xi)\right) \min & g(x, \xi) \\
\text { s.t. } x \in X(\xi) & x\}_{\xi \in \mathcal{U}},
\end{aligned}\right.
$$

where $g: \mathbb{R}^{n} \times \mathcal{U} \rightarrow \mathbb{R}$ is a single-objective function and $X(\xi) \subseteq \mathbb{R}^{n}$ denotes the feasible set with respect to the scenario $\xi \in \mathcal{U}$.

Minmax robustness for the uncertain problem $\left\{\mathcal{P}^{s-o}(\xi)\right\}_{\xi \in \mathcal{U}}$ was initially proposed in Soyster 1973, and it has been extensively studied for various problem classes and uncertainty sets, see, e.g., Ben-Tal and Nemirovski 1998, 1999, 2000. In minmax robust optimization, the following concepts of robust feasibility and robust optimality are used, see, e.g., Ben-Tal, Ghaoui, and Nemirovski 2009.

Definition 3.1.1. A solution $x \in \mathbb{R}^{n}$ is called robust feasible for the uncertain problem $\left\{\mathcal{P}^{s-o}(\xi)\right\}_{\xi \in \mathcal{U}}$, if it is feasible for all scenarios $\xi \in \mathcal{U}$, i.e., $x$ is robust feasible if and only if $x \in X(\xi)$ for all $\xi \in \mathcal{U}$. The robust feasible set is denoted as

$$
X^{\mathrm{RC}}:=\bigcap_{\xi \in \mathcal{U}} X(\xi) .
$$


Throughout this thesis, it is assumed that the robust feasible set $X^{\mathrm{RC}}$ is nonempty. The minmax robust optimal solutions for the uncertain problem $\left\{\mathcal{P}^{s-o}(\xi)\right\}_{\xi \in \mathcal{U}}$ are defined as the optimal solutions to the following deterministic problem, which is called its robust counterpart

$$
\left(\mathrm{RC}^{s-o}\right) \quad \min _{x \in X^{\mathrm{RC}}} \sup _{\xi \in \mathcal{U}} g(x, \xi) .
$$

Minmax robustness is widely considered a conservative approach to uncertainty, since minmax robust optimal solutions are those that are best in the worst-case scenarios. Conversely, an optimistic approach would be to chose an optimal solution to some scenario. It is hence of interest to compare robust optimal solutions to the optimal solutions of the scenarios. To this end, the concept of the robustness gap has been proposed and studied in Ben-Tal and Nemirovski 1998, 1999 as follows.

Definition 3.1.2. For the uncertain problem $\left\{\mathcal{P}^{s-o}(\xi)\right\}_{\xi \in \mathcal{U}}$, the single-objective robustness gap is defined as

$$
\vartheta^{s-o}:=\min _{x \in X^{\mathrm{RC}}} \sup _{\xi \in \mathcal{U}} g(x, \xi)-\sup _{\xi \in \mathcal{U}} \min _{x \in X(\xi)} g(x, \xi) .
$$

The robustness gap corresponds to the minimum distance between the robust optimal objective value and the set of optimal objective values of the scenarios, because the robust optimal objective value is as least as big as every scenario's optimal value.

One of the main results on the single-objective robustness gap is that it is zero in case of so called constraint-wise uncertainty and further assumptions, see Ben-Tal and Nemirovski 1998, Th. 2.1. The uncertainty in $\left\{\mathcal{P}^{s-o}(\xi)\right\}_{\xi \in \mathcal{U}}$ is called constraint-wise, if the uncertainty set is given as

$$
\mathcal{U}:=\mathcal{U}_{1} \times \cdots \times \mathcal{U}_{m}
$$

and if for every scenario $\xi \in \mathcal{U}$ with $\xi=\left(\xi_{1}, \ldots, \xi_{m}\right)^{T}$ and $\xi_{i} \in \mathcal{U}_{i}$, the feasible set of the scenario $\xi$ is given as

$$
X(\xi):=\left\{x \in \mathbb{R}^{n} \mid F_{i}\left(x, \xi_{i}\right) \geq 0, i=1, \ldots, m\right\},
$$

where $F_{i}: \mathbb{R}^{n} \times \mathcal{U}_{i} \rightarrow \mathbb{R}$ for all $i=1, \ldots, m$. Note that constraint-wise uncertainty occurs often in practice, e.g., when each parameter affects only one constraint and when the uncertainty set is given as a product of intervals. The additional assumptions made for the result on the zero robustness gap are the following.

\section{Assumption 3.1.1.}

(i) There exists a compact convex set $\mathfrak{X} \subseteq \mathbb{R}^{n}$ with int $(\mathfrak{X}) \neq \emptyset$ such that

$$
\bigcup_{\xi \in \mathcal{U}} X(\xi) \subseteq \mathfrak{X}
$$

(ii) The uncertainty set $\mathcal{U}$ is closed and convex, $\operatorname{int}(\mathcal{U}) \neq \emptyset$, and the uncertainty is constraint-wise. 
(iii) For every $x \in \mathfrak{X}$, the functions $f(x, \cdot): \mathcal{U} \rightarrow \mathbb{R}^{p}$ and $F(x, \cdot): \mathcal{U} \rightarrow \mathbb{R}^{m}$ are affine in $\xi$.

The result on the zero robustness gap according to Ben-Tal and Nemirovski 1998, Th. 2.1 is then given as follows.

Theorem 3.1.3. Let Assumption 3.1 .1 hold and let the function $f(\cdot, \xi): \mathcal{U} \rightarrow \mathbb{R}^{p}$ be convex and continuous and let the functions $F_{i}(\cdot, \xi): \mathcal{U} \rightarrow \mathbb{R}, i=1, \ldots, m$ be concave and continuous in $x$ for every $\xi \in \mathcal{U}$. The robust counterpart $\mathrm{RC}^{s-o}$ is then feasible if and only if all instances of the uncertain problem $\left\{\mathcal{P}^{s-o}(\xi)\right\}_{\xi \in \mathcal{U}}$ are feasible, and, in this case, the single-objective robustness gap satisfies $\vartheta^{s-o}=0$.

\section{Decision Uncertainty}

Decision uncertainty means that a vector of decision variables cannot be put into practice exactly as planned but its realization is an element of a specific set. Each realization of a solution $x \in \mathbb{R}^{n}$ comprises $x$ and an element of a fixed perturbation set $Z$, and the set of realizations of a decision vector $x \in \mathbb{R}^{n}$ is defined as $\{x\}+Z$. In this thesis, it is assumed that the perturbation set $Z$ is a compact subset of the decision space such that $0 \in Z$. A single-objective optimization problem with decision uncertainty is given as

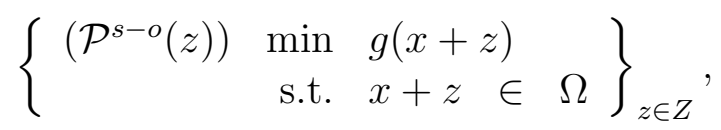

where $g: \Omega \rightarrow \mathbb{R}$ is a single-objective function and $\Omega \subseteq \mathbb{R}^{n}$ denotes the feasible set.

Minmax robust feasibility and optimality for decision uncertainty are similar to minmax robustness for parameter uncertainty. Decision uncertainty is also addressed with minmax robustness under the name implementation error, see Ben-Tal and Hertog 2011; Ben-Tal and Nemirovski 2002; Bertsimas, Nohadani, and Teo 2007, 2010a,b; Stinstra and Hertog 2008, under the name robust regularization, see, e.g., Lewis 2002; Lewis and Pang 2009, or under the general name robustness, see Das 1997. The concepts of robust feasibility and optimality with respect to decision uncertainty are given as follows.

Definition 3.1.4. A solution $x \in \mathbb{R}^{n}$ is called robust feasible for the uncertain problem $\left\{\mathcal{P}^{s-o}(z)\right\}_{z \in Z}$, if all of its realizations $x+z$, for $z \in Z$, are feasible, i.e., $x$ is robust feasible if and only if $x+z \in \Omega$ for all $z \in Z$. The decision robust feasible set is denoted as

$$
\mathcal{X}:=\left\{x \in \mathbb{R}^{n} \mid x+z \in \Omega, \text { for all } z \in Z\right\} .
$$

Throughout this thesis, it is assumed that the decision robust feasible set $\mathcal{X}$ is nonempty. The minmax decision robust optimal solutions for the uncertain problem $\left\{\mathcal{P}^{s-o}(z)\right\}_{z \in Z}$ are associated with the optimal solutions to the following deterministic problem, which is called its decision robust counterpart,

$$
\left(\mathrm{DRC}^{s-o}\right) \quad \min _{x \in \mathcal{X}} \sup _{z \in Z} g(x+z)
$$




\subsection{Deterministic Multiobjective Optimization}

A deterministic multiobjective optimization problem (MOP) is given as

$$
\begin{aligned}
(\mathcal{P}) \min & h(x) \\
\text { s.t. } & x \in \Omega
\end{aligned}
$$

where $h: \mathbb{R}^{n} \rightarrow \mathbb{R}^{p}$ with $2 \leq p$ is the objective function and $\Omega \subseteq \mathbb{R}^{n}$ is the feasible set. The set $h(\Omega):=\left\{h(x) \in \mathbb{R}^{p} \mid x \in \Omega\right\}$ is called the outcome set of the problem $\mathcal{P}$.

To compare elements of the multidimensional space $\mathbb{R}^{p}$, closed convex pointed solid cones are widely used, see, e.g., Jahn 2011.

Definition 3.2.1. A subset $K \subseteq \mathbb{R}^{p}$ is called a cone, if $\lambda k \in K$ for all $\lambda \in \mathbb{R}$ with $\lambda \geq 0$.

$A$ cone $K$ is called pointed if $K \cap(-K)=\{0\}$ and it is called solid if int $(K) \neq \emptyset$.

A given closed convex pointed solid cone $K \subseteq \mathbb{R}^{p}$ implies the following order relation on for all $y^{1}, y^{2} \in \mathbb{R}^{p}$ :

$$
\begin{aligned}
& y^{1} \leqq y^{2} \Leftrightarrow y^{1} \in\left\{y^{2}\right\}-K \\
& y^{1} \leq y^{2} \Leftrightarrow y^{1} \in\left\{y^{2}\right\}-K \backslash\{0\} \\
& y^{1}<y^{2} \Leftrightarrow y^{1} \in\left\{y^{2}\right\}-\operatorname{int}(K) .
\end{aligned}
$$

This notation is common in the literature and it is important to note that the relation $\leq$ is defined here for $\mathbb{R}^{p}$ and that the same sign is used in the one dimensional space $\mathbb{R}$ with its well-known and different meaning. In general, the meaning of the sign $\leq$ depends on the context. A frequently used choice for the ordering cone is

$$
\mathbb{R}_{\geqq}^{p}:=\left\{y \in \mathbb{R}^{p} \mid 0 \leq y_{i} \text { for all } i=1, \ldots, p\right\}
$$

and the order induced by $\mathbb{R}_{\geqq}^{p}$ is also called component-wise order. Also, the simplified notation $\mathbb{R}_{\geq}^{p}:=\mathbb{R}_{\geqq}^{p} \backslash\{0\}$ and $\mathbb{R}_{>}^{p}:=\operatorname{int}\left(\mathbb{R}_{\geqq}^{p}\right)$ is used in this thesis.

The classical optimality concept in multiobjective optimization, which is called efficiency, is given in the next definition. Efficiency is defined with respect to a given closed convex pointed solid cone $K \subseteq \mathbb{R}^{p}$. The concept of efficiency goes back to Edgeworth 1881; Pareto 1906 and it is also known as (Edgeworth-)Pareto efficiency. For comprehensive textbooks on multiobjective optimization, see, e.g., Ehrgott 2005; Jahn 2011; Miettinen 2012; Sawaragi, Nakayama, and Tanino 1985.

Definition 3.2.2. A solution $x^{*} \in \Omega$ is called [weakly/./strictly] efficient for $(\mathcal{P})$, if there is no $x \in \Omega \backslash\left\{x^{*}\right\}$ such that

$$
h(x) \in\left\{h\left(x^{*}\right)\right\}-[\operatorname{int}(K) / K \backslash\{0\} / K] .
$$

An outcome point $y^{*} \in h(\Omega)$ is called $a$ [weakly/.] non-dominated point of $\mathcal{P}$, if there exists a [weakly/.] efficient solution $x^{*} \in \Omega$ with $y^{*}=f\left(x^{*}\right)$, i.e., if there does not exist any $y \in h(\Omega)$ such that $y \in\left\{y^{*}\right\}-[\operatorname{int}(K) / K \backslash\{0\}]$. 
If the order on the objective space is the component-wise order, i.e., $K=\mathbb{R}_{\geqq}^{p}$, then a [weakly/.] non-dominated point of $\mathcal{P}$ is often also called a [weak/.] Pareto point. The set of Pareto points is called the Pareto set of $\mathcal{P}$ and the set of efficient points is called the efficient set of $\mathcal{P}$ or equivalently the set of efficient solutions. Furthermore, in case of $K=\mathbb{R}_{\geqq}^{p}$ the ideal point $z_{\mathcal{P}}^{I} \in \mathbb{R}^{p}$ of $\mathcal{P}$ is defined as the vector that consists in every component of the infimum of the outcome set of $\mathcal{P}$

$$
\left(z_{\mathcal{P}}^{I}\right)_{i}:=\inf _{x \in \Omega} h_{i}(x) .
$$

In addition to the concept of efficiency, there are concepts of approximate efficiency in the literature, see, e.g., Durea 2007 and the references therein, and the following concept goes back to Kutateladze 1979.

Definition 3.2.3. Let $k^{0} \in K \backslash\{0\}$ and $\varepsilon>0$ be given. The point $x^{*} \in \Omega$ is a [weakly $/ \cdot /$ strictly] $\left(\varepsilon, k^{0}\right)$-minimal solution for $(\mathcal{P})$, if there exists no $x \in \Omega$ such that

$$
h(x) \in\left\{h\left(x^{*}\right)-\varepsilon k^{0}\right\}-[\operatorname{int}(K) / K \backslash\{0\} / K] .
$$

A notion of convexity for functions that map to the multidimensional space $\mathbb{R}^{p}$ ordered by a given closed convex pointed solid cone $K$, is given as follows, see, e.g., Kuroiwa 1996, Def. 2.1.

Definition 3.2.4. Let $Y \subseteq \mathbb{R}^{n}$ be a convex set. A map $h: Y \rightarrow \mathbb{R}^{k}$ is called $K$-convex, if for all $x^{1}, x^{2} \in Y$ and $\lambda \in[0,1]$ it holds that

$$
\lambda h\left(x^{1}\right)+(1-\lambda) h\left(x^{2}\right) \in\left\{h\left(\lambda x^{1}+(1-\lambda) x^{2}\right)\right\}+K .
$$

In case of $K=\mathbb{R}_{\geqq}^{p}, K$-convexity is also shortly referred to as convexity. Note that the MOP $\mathcal{P}$ is called convex, if $K=\mathbb{R}_{\geqq}^{p}$, the objective function $h$ is convex and if the feasible set $\Omega$ is convex.

Among the solution methods for MOPs, one widely used is the weighted-sum scalarization, see, e.g., Geoffrion 1968. In this thesis, the weighted sum scalarization is applied in the case that the objective space $\mathbb{R}^{p}$ is considered a normed space that is equipped with the ordering cone $\mathbb{R}_{\geqq}^{p}$. The primal norm on $\mathbb{R}^{p}$ is then denoted as $\|\cdot\|: \mathbb{R}^{p} \rightarrow \mathbb{R}$ and its dual norm is denoted as $\|\cdot\|_{*}: \mathbb{R}^{p} \rightarrow \mathbb{R}$. Correspondingly, the primal space is given as $\left(\mathbb{R}^{p},\|\cdot\|\right)$ and its dual space is given as $\left(\mathbb{R}^{p},\|\cdot\|_{*}\right)$.

To keep notation simple, the weighted-sum scalarization for the normed space $\left(\mathbb{R}^{p},\|\cdot\|\right)$ with the ordering cone $\mathbb{R}_{\geqq}^{p}$ is next recalled, and for a more general study of the weightedsum scalarization, see, e.g., Luc 1989.

The weighted-sum scalarization transforms an MOP into a single-objective problem by applying linear functions to the objective function. The linear functions used are called weights and they are elements of $\left(\mathbb{R}^{p},\|\cdot\|_{*}\right)$, i.e., the dual space of $\left(\mathbb{R}^{p},\|\cdot\|\right)$. The weights are exactly the elements of the dual cone of the ordering cone $\mathbb{R}_{\geqq}^{p} \subseteq\left(\mathbb{R}^{p},\|\cdot\|\right)$, which is given as

$$
\left\{\lambda \in\left(\mathbb{R}^{p},\|\cdot\|_{*}\right) \mid \lambda^{T} k \geq 0 \text { for all } k \in \mathbb{R}_{\geqq}^{p}\right\}
$$


i.e., the dual cone to the cone $\mathbb{R}_{\geqq}^{p} \subseteq\left(\mathbb{R}^{p},\|\cdot\|\right)$ is $\mathbb{R}_{\geqq}^{p} \subseteq\left(\mathbb{R}^{p},\|\cdot\|_{*}\right)$.

Applying a weight $\lambda$ of the dual cone $\mathbb{R}_{\geqq}^{p} \subseteq\left(\mathbb{R}^{p},\|\cdot\|_{*}\right)$ to the MOP $\mathcal{P}$ yields the single-objective problem

$$
\begin{array}{rll}
(\mathcal{P}(\lambda)) & \min & \lambda^{T} h(x) \\
\text { s.t. } & x \in \Omega
\end{array} .
$$

The following relation between weakly efficient solutions and the solutions of the weightedsum scalarization goes back to Luc 1989, Th.2.10.

Proposition 3.2.5. If $\mathcal{P}$ is a convex $M O P$, then a solution $x^{*} \in \Omega$ is a weakly efficient solution of $\mathcal{P}$ if and only if there exists $\lambda \in \mathbb{R}_{\geqq}^{p} \subseteq\left(\mathbb{R}^{p},\|\cdot\|_{*}\right)$ with $\lambda \neq 0$ such that $x^{*}$ is an optimal solution to $\mathcal{P}(\lambda)$.

\subsection{Set-Valued Optimization}

A set-valued optimization problem is given as

$$
\begin{aligned}
\left(\mathcal{P}^{\text {set }}\right) \min & H(x) \\
\text { s.t. } & x \in \Omega,
\end{aligned}
$$

where $H: \mathbb{R}^{n} \rightrightarrows \mathbb{R}^{p}$ is a set-valued map and $\Omega \subseteq \mathbb{R}^{n}$ is the feasible set. Throughout this thesis it is assumed that the feasible set $\Omega$ is nonempty and that the sets $H(x)$ are nonempty for all $x \in \Omega$.

In order to define an optimality concept for the set-valued problem $\mathcal{P}^{\text {set }}$, a concept for comparing the sets that are the images of the objective function needs to be defined. In the so-called set approach order relations are used to compare sets, see Eichfelder and Jahn 2012; Ha and Jahn 2011; Kuroiwa 1998. In this thesis, the $u$-type less order relation $\preccurlyeq_{K}$ is used to compare sets by means of a given closed convex pointed solid cone $K \subseteq \mathbb{R}^{p}$. The order relation $\preccurlyeq_{K}$ and the related set relations $\preccurlyeq_{K \backslash\{0\}}$ and $\preccurlyeq_{\operatorname{int}(K)}$ are defined as follows for all sets $A, B \subseteq \mathbb{R}^{p}$, see, e.g., Ha and Jahn 2011, Def. 3.2.

$$
\begin{aligned}
& A \preccurlyeq K \quad B \Leftrightarrow A \subseteq\{B\}-K \\
& A \preccurlyeq K \backslash\{0\} \quad B \Leftrightarrow A \subseteq\{B\}-K \backslash\{0\} \\
& A \preccurlyeq \operatorname{int}(K) \quad B \Leftrightarrow A \subseteq\{B\}-\operatorname{int}(K) \text {. }
\end{aligned}
$$

These set relations can equivalently be expressed as follows by using the order relations for points in (3.8).

$$
A[\preccurlyeq \operatorname{int}(K) / \preccurlyeq K \backslash\{0\} / \preccurlyeq K] B \Leftrightarrow(\forall a \in A \exists b \in B: a[</ \leq / \leqq] b) .
$$

The following concept of optimality for the set-valued optimization problem $\mathcal{P}^{\text {set }}$, see, e.g., Rodríguez-Marín and Sama 2007, is used in this thesis.

Definition 3.3.1. The element $x^{*} \in \Omega$ is called strictly optimal solution of the setvalued optimization problem $\mathcal{P}^{\text {set }}$ w.r.t. $\preccurlyeq$, where $\preccurlyeq \in\left\{\preccurlyeq_{K}, \preccurlyeq_{K \backslash\{0\}}, \preccurlyeq_{\operatorname{int}(K)}\right\}$, if there exists no $x \in \Omega \backslash\left\{x^{*}\right\}$ with $H(x) \preccurlyeq H\left(x^{*}\right)$. 
In the multidimensional space $\mathbb{R}^{p}$ with $p \geq 2$, there exists no universal concept of the supremum of a set. To this end, the concept of the supremal set is used, which is introduced and thoroughly studied in Löhne 2011; Nieuwenhuis 1980.

Definition 3.3.2. For a bounded nonempty subset $A \subsetneq \mathbb{R}^{p}$ the supremal set of $A$ is defined as

$$
\operatorname{Sup}(A)=\{y \in \operatorname{cl}(A-K) \mid(\{y\}+\operatorname{int}(K)) \cap \operatorname{cl}(A-K)=\emptyset\}
$$

Also note that, by Löhne 2011, Cor. 1.48(iv), the supremal set of a bounded nonempty subset $A \subsetneq \mathbb{R}^{k}$ can be easily imagined, because it holds

$$
\operatorname{Sup}(A)=\operatorname{bd}(A-K) \text {. }
$$

A notion of convexity for set-valued functions is recalled next and for detailed studies thereof see, e.g., Benoist and Popovici 2003; Kuroiwa 1996.

Definition 3.3.3. Let $Y \subseteq \mathbb{R}^{n}$ be a convex set. A set-valued map $H: Y \rightrightarrows \mathbb{R}^{p}$ is called $K$-convex, if for all $x^{1}, x^{2} \in Y$ and $\lambda \in[0,1]$ it holds that $\lambda H\left(x^{1}\right)+(1-\lambda) H\left(x^{2}\right) \subseteq$ $H\left(\lambda x^{1}+(1-\lambda) x^{2}\right)+K$.

In this thesis, semicontinuity of set-valued maps $H: \Omega \rightrightarrows \mathbb{R}^{p}$ is investigated for the case that the set $\Omega$ is equipped with a norm, e.g., the set $\Omega$ can be considered a subset of a normed vector space. Therefore, each subset $\Omega \subseteq \mathbb{R}^{p}$ can be considered a metric space and a standard definition of lower and upper semicontinuity can be applied, see, e.g., Khan, Tammer, and Zălinescu 2015, p. 3.1.1 and Aubin and Frankowska 1990, Section 1.4 .

Definition 3.3.4. Let $\Omega$ be a metric space and let $H: \Omega \rightrightarrows \mathbb{R}^{p}$ be a set-valued map.

(a) $H$ is called lower semicontinuous at $x^{0} \in \Omega$, if for all open sets $V \subseteq \mathbb{R}^{p}$ with $H\left(x^{0}\right) \cap V \neq \emptyset$ there is a neighborhood $U$ of $x^{0}$ such that $H(x) \cap V \neq \emptyset$ for all $x \in U$. $H$ is lower semicontinuous if it is lower semicontinuous at any $x^{0} \in \Omega$.

(b) $H$ is called upper semicontinuous at $x^{0} \in \Omega$ if for all open sets $V \subseteq \mathbb{R}^{p}$ with $H\left(x^{0}\right) \subseteq V$ there is a neighborhood $U$ of $x^{0}$ such that $H(x) \subseteq V$ for all $x \in U$. $H$ is upper semicontinuous if it is upper semicontinuous at any $x^{0} \in \Omega$.

\subsection{Minmax Robustness for Parameter Uncertainty in Multiobjective Optimization}

A multiobjective optimization problem with parameter uncertainty is given as

$$
\left\{\begin{array}{ll}
(\mathcal{P}(\xi)) & \min \\
\text { s.t. } & x \in X(\xi) \xi)
\end{array}\right\}_{\xi \in \mathcal{U}},
$$


where $f: \mathbb{R}^{n} \times \mathcal{U} \rightarrow \mathbb{R}^{p}$ and $X(\xi) \subseteq \mathbb{R}^{n}$ denotes the feasible set with respect to the scenario $\xi \in \mathcal{U}$.

Two frequently studied minmax robustness concepts for MOPs with parameter uncertainty such as $\{\mathcal{P}(\xi)\}_{\xi \in \mathcal{U}}$ are point-based robust efficiency and set-based robust efficiency and these two concepts are also considered in the publications contributing to this thesis.

Both robustness concepts use the same definition of robust feasibility as in singleobjective minmax robust optimization, see Definition 3.1.1. The two concepts are therefore distinguished by their definitions of worst-case outcomes in the objective space. The robust counterpart corresponding to point-based robust efficiency is an MOP, whereas the robust counterpart corresponding to set-based robust efficiency is a set-valued optimization problem.

\subsubsection{Point-Based Robust Efficiency}

The concept of point-based robust efficiency goes back to Kuroiwa and Lee 2012.

This concept extends the minmax robust counterpart $\mathrm{RC}^{s-o}$ as given in (3.2) to MOPs with parameter uncertainty, by replacing every component of the objective function with its supremum over the scenarios.

A worst-case objective function $f^{\mathrm{RC}}: X^{\mathrm{RC}} \rightarrow \mathbb{R}^{p}$ is then defined as

$$
f_{i}^{\mathrm{RC}}(x):=\sup _{\xi \in \mathcal{U}} f_{i}(x, \xi)
$$

for all $1 \leq i \leq p$ and for all robust feasible solutions $x \in X^{\mathrm{RC}}$.

The corresponding point-based robust counterpart is given as

$$
\left(\mathrm{RC}^{\text {point }}\right) \quad \min _{x \in X^{\mathrm{RC}}} f^{\mathrm{RC}}(x) .
$$

and a solution $x \in \mathbb{R}^{n}$ is considered a point-based robust [weakly/./strictly] efficient solution of $\{\mathcal{P}(\xi)\}_{\xi \in \mathcal{U}}$ if it is a [weakly/./strictly] efficient solution of $\mathrm{RC}^{\text {point }}$.

\subsubsection{Set-Based Robust Efficiency}

The concept of set-based robust efficiency goes back to Ehrgott, Ide, and Schöbel 2014.

This concept extends the minmax robust counterpart $\mathrm{RC}^{s-o}$ as given in (3.2) to MOPs with parameter uncertainty, by taking into account all scenarios and comparing solutions by their entire sets of outcomes. Thus the robust counterpart is defined as a set-valued optimization problem.

The objective function for the robust counterpart is defined as $f_{\mathcal{U}}: X^{\mathrm{RC}} \rightrightarrows \mathbb{R}^{p}$ with

$$
f_{\mathcal{U}}(x):=\{f(x, \xi) \mid \xi \in \mathcal{U}\}
$$

for all robust feasible solutions $x \in X^{\mathrm{RC}}$.

The corresponding point-based robust counterpart is given as

$$
\begin{array}{lll}
\left(\mathrm{RC}^{\text {set }}\right) & \min & f_{\mathcal{U}}(x) \\
\text { s. t. } & x \in X^{\mathrm{RC}},
\end{array}
$$


and a solution $x \in \mathbb{R}^{n}$ is considered a set-based robust [weakly/./strictly] efficient solution of $\{\mathcal{P}(\xi)\}_{\xi \in \mathcal{U}}$ if it is a strictly optimal solution of the set-valued problem $\mathrm{RC}^{\text {set }}$ with respect to $\left[\preceq_{\operatorname{int}(K)} / \preceq_{K \backslash\{0\}} / \preceq_{K}\right]$.

\subsubsection{Relation between the two Concepts}

The two concepts point-based robust efficiency and set-based robust efficiency coincide under the condition that the uncertainty is objective-wise. Objective-wise uncertainty is similar to constraint-wise uncertainty as given in Section 3.1, see (3.3) and (3.4), but objective-wise uncertainty affects the objective function instead of the constraints.

The uncertainty in $\{\mathcal{P}(\xi)\}_{\xi \in \mathcal{U}}$ is called objective-wise, if the uncertainty set is given as

$$
\mathcal{U}:=\mathcal{U}_{1} \times \cdots \times \mathcal{U}_{p}
$$

and if for every scenario $\xi \in \mathcal{U}$ with $\xi=\left(\xi_{1}, \ldots, \xi_{p}\right)^{T}$ and $\xi_{i} \in \mathcal{U}_{i}$, and every solution $x \in X(\xi)$, the objective function $f(x, \xi)$ is given as

$$
f(x, \xi):=\left(f_{1}\left(x, \xi_{1}\right), \cdots, f_{p}\left(x, \xi_{p}\right)\right)^{T} .
$$

Note that objective-wise uncertainty occurs often in practice, e.g., when each parameter affects only one component of the objective function and when the uncertainty set is given as a product of intervals.

The following result, which corresponds to Ehrgott, Ide, and Schöbel 2014, Th. 5.4, shows the equivalence of point-based and set-based robust efficiency under objective-wise uncertainty.

Theorem 3.4.1. If the uncertainty in $\{\mathcal{P}(\xi)\}_{\xi \in \mathcal{U}}$ is objective-wise and if $\max _{\xi_{i} \in \mathcal{U}_{i}} f_{i}\left(x, \xi_{i}\right)$ exists for all $i=1, \ldots, p$, then every solution $x \in X^{\mathrm{RC}}$ is a point-based robust efficient solution of $\{\mathcal{P}(\xi)\}_{\xi \in \mathcal{U}}$ if and only if it is a set-based robust efficient solution of $\{\mathcal{P}(\xi)\}_{\xi \in \mathcal{U}}$. 


\section{Summary of the publications}

In the following, the four publications contributing to this thesis are summarized.

In Section 4.1, an overview on the results in Eichfelder, Krüger, and Schöbel 2017 is given. A minmax robustness concept for uncertain multiobjective problems with decision uncertainty is proposed. The robust counterpart is introduced as a set-valued optimization problem and it is shown that two different set-valued maps can be used as the objective function of the robust counterpart, yielding the same set of solutions. Analytical properties of the two set-valued maps are studied and solution approaches for three problem classes are investigated. The presented robustness concept for decision uncertainty is also compared to other robustness concepts in the literature.

In Section 4.2, the contents of the publication Krüger, Schöbel, and Wiecek 2017 are summarized. A robustness gap for robust multiobjective optimization is introduced and the multiobjective robustness gap is defined as the minimal distance between the Pareto sets of the scenarios and the robust Pareto set, i.e., the Pareto set of the robust counterpart that is an MOP. The gap is hard to compute and lower and upper bounds are presented for the case of convex uncertain MOPs. Two conditions for the gap to be zero are given, one for convex MOPs, extending a well-known major result on the singleobjective gap, and one for linear MOPs. For linear MOPs, a DC optimization problem whose optimal objective value corresponds to the lower bound is also presented.

In Section 4.3, an application of robust multiobjective optimization to a biobjective problem with decision uncertainty is investigated that is of interest for the commercial cultivation of potted plants. The aim is to choose a type of planter pot and a mixture of growth substrate such that a economic and an environmental objective function are optimized. The problem is addressed first as a deterministic problem and second, parameter and decision uncertainty are taken into account. For both cases a solution approach is given. These solutions appraoches are applied within a case study and the deterministic efficient solutions and the (decision) robust efficient to the problem are calculated and compared.

The content of this research is divided into two parts: the practical part that is given in the publication Krüger, Castellani, Geldermann, and Schöbel 2018 and the theoretical part that is given in the publication Krüger 2018. The practical part consists of a detailed motivation of the problem's relevance for the horticulture industry as well as the computation and analysis of the deterministic and the robust efficient solutions for a case study. The theoretical part provides the background of the solution techniques that are used in the practical part. Analytical descriptions of the deterministic efficient solutions and the robust efficient solutions are presented and extensions of the presented solution approaches to further uncertain biobjective problems are given. 


\subsection{Decision Uncertainty in Multiobjective Optimization}

In this section, the publication Eichfelder, Krüger, and Schöbel 2017 is summarized, which is joint work with Prof. Dr. Gabriele Eichfelder and Prof. Dr. Anita Schöbel. The final publication is available at link.springer.com.

As a motivation to consider decision uncertainty in multiobjective optimization, two applications are briefly stated, one being an application in the Lorentz force velocimetry framework and the other one being the peat-and-pots problem that in studied in detail in Krüger 2018; Krüger, Castellani, Geldermann, and Schöbel 2018 and that is summarized in Section 4.3.

A multiobjective optimization problem with decision uncertainty is considered as

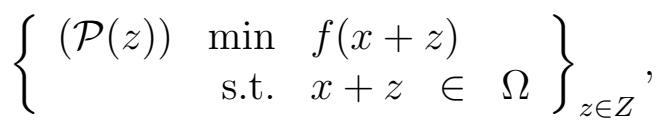

where $f: \Omega \rightarrow \mathbb{R}^{p}$ and $\Omega \subseteq \mathbb{R}^{n}$ denotes the feasible set. Throughout the publication it is assumed that the perturbation set $Z$ is compact with $0 \in Z$.

To define decision robust feasibility for the uncertain problem $\{\mathcal{P}(z)\}_{z \in Z}$, the concept from single-objective robust optimization is used, see Section 3.1 Definition 3.1.4, since feasibility is not affected by the dimension of the objective space. Correspondingly, a point $x \in \mathbb{R}^{n}$ is called decision robust feasible for $\{\mathcal{P}(z)\}_{z \in Z}$ if all realizations of $x$ are feasible, i.e., if $\{x+z \mid z \in Z\} \subseteq \Omega$, and the set of decision robust feasible solutions is denoted as $\mathcal{X}$.

As an optimality concept, the concept of decision robust efficiency is then introduced. The idea is to compare solutions by their entire outcome sets, i.e., by considering for each solution $x \in \mathcal{X}$ the outcome set of all its realizations, which is given as

$$
f_{Z}(x):=\{f(x+z) \mid z \in Z\} .
$$

Definition 4.1.1 (Eichfelder, Krüger, and Schöbel 2017, Def. 2). A solution $x^{*} \in \mathcal{X}$ is called a decision robust [weakly $/ \cdot /$ strictly] efficient solution of $\{\mathcal{P}(z)\}_{z \in Z}$, if there is no $x \in \mathcal{X} \backslash\left\{x^{*}\right\}$ with the property

$$
f_{Z}(x) \subseteq f_{Z}\left(x^{*}\right)-[\operatorname{int}(K) / K \backslash\{0\} / K] .
$$

This definition is motivated in two ways. One the one hand, considering the definition of efficiency for deterministic MOPs, see Definition 3.2.2, and replacing for every $x \in \mathcal{X}$ the point $f(x)$ by the set $f_{Z}(x)$, yields Definition 4.1.1. This motivation also corresponds to the idea behind set-based robust efficiency for MOPs with parameter uncertainty as proposed in Ehrgott, Ide, and Schöbel 2014.

On the other hand, the concept of decision robust efficiency can equivalently be obtained by defining the robust counterpart of $\{\mathcal{P}(z)\}_{z \in Z}$ as the set-valued problem

$$
\text { (DRC) } \min _{x \in \mathcal{X}} f_{Z}(x)
$$


and applying the optimality concept stated in Definition 3.3.1, see (Eichfelder, Krüger, and Schöbel 2017, Th. 5).

Recall that minmax robustness for single-objective optimization is a worst-case approach, and it involves minimizing the supremum over all scenarios, see (3.2). To see that decision robust efficiency is a minmax robustnes concept for multiobjective optimization, it is shown that the set-valued objective function in (4.3) can be replaced by a supremal set, which is a concept of a supremum in multidimensional space, see Definition 3.3.2. The corresponding set-valued optimization problem is then given as

$$
\min _{x \in X} f_{\text {Sup }}(x) \quad \text { with } \quad f_{\text {Sup }}: \mathcal{X} \rightrightarrows \mathbb{R}^{k}, x \mapsto \operatorname{Sup}\left(f_{Z}(x)\right)
$$

The problems (4.3) and (4.4) are equivalent in the sense that their optimal solutions coincide, which are exactly the decision robust efficient solutions to $\{\mathcal{P}(z)\}_{z \in Z}$.

Theorem 4.1.2 (Eichfelder, Krüger, and Schöbel 2017, Th. 9). Let $f$ be continuous. A point $x^{*} \in \mathcal{X}$ is a decision robust [weakly/./strictly] efficient solution of $\{\mathcal{P}(z)\}_{z \in Z}$ if and only if $x^{*} \in \mathcal{X}$ is a strictly optimal solution for the set-valued optimization problem (4.4) w.r.t. $[\preccurlyeq \operatorname{int}(K) / \preccurlyeq K \backslash\{0\} / \preccurlyeq K]$.

Because the two set-valued problems (4.3) and (4.4) both lead to the same set of solutions, namely the decision robust efficient solutions, analytical properties of the two set-valued objective functions are investigated such as convexity and lower and upper semicontinuity.

As shown in (Eichfelder, Krüger, and Schöbel 2017, Prop. 12), the decision robust feasible set is convex if the feasible set $\Omega$ and the perturbation set $Z$ are convex. The objective function of (4.3), $f_{Z}$, is $K$-convex if the map $f$ is $K$-convex. However, the objective function of $(4.4), f_{\text {Sup }}$, is not convex under this assumption.

For the objective function of (4.3), $f_{Z}$, lower and upper semicontinuity are shown under the assumption that the the map $f$ is continuous in (Eichfelder, Krüger, and Schöbel 2017, Prop. 14, Prop. 15). The latter assumption is passable according to (Eichfelder, Krüger, and Schöbel 2017, Ex. 2) where it is illustrated that the set-valued map $f_{Z}$ is not necessarily lower or upper semicontinuous if there is at least one point where $f$ is not continuous.

The alternative objective function of (4.4) $f_{\text {Sup }}$ that maps solutions to supremal sets is also lower semicontinuous if $f$ is continuous as shown in (Eichfelder, Krüger, and Schöbel 2017, Prop. 17). However, continuity of $f$ does not imply upper semicontinuity of $f_{\text {Sup }}$, which is illustrated in (Eichfelder, Krüger, and Schöbel 2017, Ex. 3).

As the main result on the continuity of the map $f_{\text {Sup }}$, it is then shown that if two solutions are close to each other, the supremal sets of their outcome sets are also within an arbitrarily small neighborhood of each other. Note that in the next result the open ball around 0 is denoted as $B(0, \varepsilon):=\left\{y \in \mathbb{R}^{p} \mid\|y\|<\varepsilon\right\}$.

Theorem 4.1.3 (Eichfelder, Krüger, and Schöbel 2017, Th. 18). Let $f: \Omega \rightarrow \mathbb{R}^{p}$ be continuous. Then for each $x^{0} \in \Omega$ and $\varepsilon>0$ there exists $\delta>0$ such that

$$
\operatorname{Sup}\left(f_{Z}(x)\right) \subseteq \operatorname{Sup}\left(f_{Z}\left(x^{0}\right)\right)+B(0, \varepsilon)
$$


for all $x \in \Omega$ with $\left\|x^{0}-x\right\|<\delta$.

The concept of decision robust efficiency is not only interesting because of its practical motivation and its relation to deterministic multiobjective and set optimization, but it also constitutes a link two robustness concepts in the literature. It can be considered an extension of a single-objective robustness concept and it can be considered as a special case of a multiobjective robustness concept for parameter uncertainty. In (Eichfelder, Krüger, and Schöbel 2017, Prop. 19), it is first related to a robustness concept for decision uncertainty in single-objective optimization and it is shown that decision robust efficiency can be considered a generalization of the single-objective concept. The setvalued objective function $f_{\text {Sup }}$ corresponds to a supremum in the single-objective case and the concepts of robust optimality, see Section 3.1 and decision robust efficiency, see Definition 4.1.1, are closely related and even coincide if the objective function $f$ is continuous. If $f$ is not continuous then different implications hold for weakly and strictly robust efficiency as stated in (Eichfelder, Krüger, and Schöbel 2017, Rem. 2): every decision robust [weakly/.] efficient solution is also robust optimal and if there is a unique robust optimal solution, then it is also decision robust strictly efficient.

Decision robust efficiency is secondly compared with the minmax robustness concept set-based robust efficiency for parameter uncertainty in multiobjective optimization, see Section 3.4, and Problem (3.13). By (Eichfelder, Krüger, and Schöbel 2017, Prop. 21), every MOP with decision uncertainty can be rewritten as an MOP with parameter uncertainty such that the decision robust efficient solutions to the former are exactly the set-based robust efficient solutions to the latter.

As a consequence of this, MOPs with decision uncertainty can be regarded as a special case of MOPs with parameter uncertainty and the results of Ehrgott, Ide, and Schöbel 2014 apply. Decision uncertainty in MOPs implies more structure than parameter uncertainty and this structure is reflected in properties of decision uncertainty that do not hold for parameter uncertainty. A significant difference between the two types of uncertainty is the following: For MOPs with parameter uncertainty, no method is known to determine all set-based robust efficient solutions, not even in the case of linear objective functions, see (Ehrgott, Ide, and Schöbel 2014, p. 30). However, for MOPs with decision uncertainty, all decision robust efficient solutions can be determined by methods from deterministic multiobjective optimization if the objective function is linear. It is shown that the decision robust efficient solutions for $\{\mathcal{P}(z)\}_{z \in Z}$ correspond to the efficient solutions of the deterministic problem

$$
\left(\left.\mathcal{P}\right|_{\mathcal{X}} \quad \min _{x \in \mathcal{X}} f(x)\right.
$$

Theorem 4.1.4 (Eichfelder, Krüger, and Schöbel 2017, Th. 23). Let $f: \Omega \rightarrow \mathbb{R}^{p}$ be linear. Then for each decision robust feasible solution $x \in \mathcal{X}$

$$
\begin{array}{r}
x \text { is }\left[\text { weakly/·strictly] efficient for }\left(\left.\mathcal{P}\right|_{\mathcal{X}}\right)\right. \\
\Leftrightarrow \quad x \text { is decision robust }\left[\text { weakly/·/strictly] efficient for }\{\mathcal{P}(z)\}_{z \in Z} .\right.
\end{array}
$$


Solution approaches for MOPs with decision uncertainty that are based on methods for deterministic MOPs are of particular interest, because deterministic MOPs are generally easier to solve than set-valued optimization problems.

Two additional types of objective functions are investigated in this publication, Lipschitz continuous objective functions and monotonic objective functions. If the objective function $f$ is Lipschitz continuous then every decision robust efficient solution is also approximately efficient for the deterministic problem $\left.\mathcal{P}\right|_{\mathcal{X}}$, i.e., a necessary condition for decision robust efficiency is proved. Note that the closed ball around 0 is denoted as $\bar{B}(0, \varepsilon):=\left\{y \in \mathbb{R}^{p} \mid\|y\| \leq \varepsilon\right\}$ in the next result.

Theorem 4.1.5 (Eichfelder, Krüger, and Schöbel 2017, Th. 25). Let $f: \Omega \rightarrow \mathbb{R}^{p}$ be Lipschitz continuous with Lipschitz constant $L>0$ and $Z \neq\{0\}$. Furthermore, set

$$
\tilde{L}:=L \cdot \max _{z \in Z}\|z\| \quad \text { and } \quad S:=\bigcap_{h \in \bar{B}(0, \tilde{L})}\{h\}+K .
$$

If $x^{*} \in \mathcal{X}$ is decision robust [weakly/·/strictly] efficient for $\{\mathcal{P}(z)\}_{z \in Z}$, then there exists no $\tilde{x} \in \mathcal{X}$ and no $s \in S$ such that $f(\tilde{x}) \in\left\{f\left(x^{*}\right)-s\right\}-[\operatorname{int}(K) / K \backslash\{0\} / K]$. Hence, $x^{*}$ is an [weakly/./strictly] $\left(\varepsilon, k^{0}\right)$-minimal solution for $(\mathcal{P} \mid \mathcal{X})$, i.e., $\min _{x \in \mathcal{X}} f(x)$, where

$$
k^{0}=\frac{s}{\|s\|} \quad \text { and } \quad \varepsilon=\|s\|
$$

for all $s \in S$.

The final result for determining decision robust efficient solutions for specific problem classes is given for the case of monotonic objective functions. If the objective function $f$ is monotonically increasing in at least one component and monotonically decreasing in another component, then a sufficient condition for decision robust efficiency is given as follows.

Theorem 4.1.6 (Eichfelder, Krüger, and Schöbel 2017, Th. 27). Let $\mathcal{X} \subseteq \Omega \subseteq \mathbb{R}^{n}$ and let $x \in \mathcal{X}$ be such that for all $y \in \mathcal{X}$

$$
x \neq y \quad \Rightarrow \quad x[\leq /</ \leq] y \text { or } y[\leq /</ \leq] x
$$

and let $Z \subseteq \mathbb{R}^{n}$ have the property

$$
z^{1} \neq z^{2} \quad \Rightarrow \quad z^{1}[\leq /</ \leq] z^{2} \quad \text { or } \quad z^{2}[\leq /</ \leq] z^{1}
$$

for all $z^{1}, z^{2} \in Z$. Let $p \geq 2$ and let $f: \mathbb{R}^{n} \rightarrow \mathbb{R}^{k}$ have the property that there exist $j, l \in\{1, \ldots, k\}, j \neq l$, such that

(a) $f_{j}: \mathbb{R}^{n} \rightarrow \mathbb{R}$ is [./strictly/strongly $]$ increasing on $\mathcal{X}$

(b) $f_{l}: \mathbb{R}^{n} \rightarrow \mathbb{R}$ is $[. /$ strictly/strongly $]$ decreasing on $\mathcal{X}$.

Then $x$ is decision robust [weakly/strictly/strictly]-efficient. 
A direct consequence of this is the next corollary in which a sufficient condition for decision robust efficiency is given for the case that the feasible set is an interval.

Corollary 4.1.7 (Eichfelder, Krüger, and Schöbel 2017, Cor. 28). Let $Z \subseteq \mathbb{R}$ and $\mathcal{X} \subseteq \Omega \subseteq \mathbb{R}$ be compact intervals and $f: \mathbb{R} \rightarrow \mathbb{R}^{p}$ be such that $f_{1}: \mathbb{R} \rightarrow \mathbb{R}$ is $[. /$ strictly] increasing and $f_{2}: \mathbb{R} \rightarrow \mathbb{R}$ is $[. /$ strictly $]$ decreasing.

Then all $x \in \mathcal{X}$ are decision robust [weakly/strictly]-efficient.

These two results for monotonic objective functions are illustrated by an example in (Eichfelder, Krüger, and Schöbel 2017, Ex. 4). The results are of interest for applications in production planning where one objective function represents the costs of a production process while another objective function represents the environmental impacts. In particular, the corollary can be used to adjust the results of the third publication of this thesis for applications where traditional input materials of a production process are substituted by alternative that are more eco-friendly and more expensive than the traditional materials, see Section 4.3.

In summary, decision robust efficiency is introduced as a solution concept for MOPs with decision uncertainty and it is shown that this concept can be considered as a minmax robustness concept, because the decision robust efficient solutions are the optimal solutions of a set optimization problem defined by minimizing supremal sets over the set of solutions that are feasible for all their realizations. The concept is also shown to fit to existing minmax robustness concepts in the literature as it might be considered a generalization of one concept and as a special case of another concept.

The concept of decision robust efficiency is nevertheless worth studying in its own right. The two set-valued maps that can equivalently be considered as the objective functions of the decision robust counterpart are shown to possess several continuity properties, which are an important for solution methods to optimization problems such as decent methods. Moreover, solution approaches for three specific classes of MOPs with decision uncertainty are presented.

\section{Own Contribution}

The introduction of the concept decision robust efficiency and its connection to robustness for parameter uncertainty, set-valued optimization and supremal sets is joint work of all authors of the publication. The largest part of the writing of this publication was done by the author of this thesis. The author of this thesis contributed to most of the proofs and examples in this publication. In particular, the proof of the upper semicontinuity of the function $f_{Z}$, the proofs of the semicontinuity of the function $f_{\text {Sup }}$, and the proof that the decision robust counterpart can be considered as an MOP in case of linear objective functions, were mostly done by the author of this thesis with the advice of the coauthors. 


\subsection{The Robustness Gap for Uncertain Multiobjective Optimization}

The publication Krüger, Schöbel, and Wiecek 2017 is summarized next, which is joint work with Prof. Dr. Anita Schöbel and Prof. Dr. Margaret Wiecek.

Throughout the publication, the $p$-dimensional vector space $\mathbb{R}^{p}$ is considered as a normed space with norm $\|\cdot\|: \mathbb{R}^{p} \rightarrow \mathbb{R}$. The dual norm of $\|\cdot\|$ is denoted as $\|\cdot\|_{*}: \mathbb{R}^{p} \rightarrow \mathbb{R}$. The primal space is then referred to as $\left(\mathbb{R}^{p},\|\cdot\|\right)$ and the dual space is referred to as $\left(\mathbb{R}^{p},\|\cdot\|_{*}\right)$. To keep notation short, the primal space is also simply written as $\mathbb{R}^{p}$. It is also assumed that the primal space is ordered by the Pareto cone $\mathbb{R}_{\geqq}^{p}$.

The uncertain multiobjective optimization problem

$$
\left\{\begin{array}{rlrl}
(\mathcal{P}(\xi)) & \min _{x} & f(x, \xi) & \\
& \text { s. t. } & F(x, \xi) & \geqq 0 \\
& & x & \in \mathbb{R}^{n}
\end{array}\right\}_{\xi \in \mathcal{U}}
$$

is considered, see Section 3.4, and the uncertainty set $\mathcal{U}$ is assumed to be a compact subset of a finite dimensional vector space. For each scenario $\xi \in \mathcal{U}$ the feasible set is denoted as

$$
X(\xi):=\left\{x \in \mathbb{R}^{n} \mid F(x, \xi) \geqq 0\right\}
$$

and each set $X(\xi)$ is assumed to be compact.

Throughout the paper, the following Continuity Assumption is made: The functions $f(\cdot, \xi): \mathbb{R}^{n} \rightarrow \mathbb{R}^{p}$ and $F(\cdot, \xi): \mathbb{R}^{n} \rightarrow \mathbb{R}^{m}$ are assumed to be continuous for all $\xi \in \mathcal{U}$ and the functions $f(x, \cdot): \mathcal{U} \rightarrow \mathbb{R}^{p}$ and $F(x, \cdot): \mathcal{U} \rightarrow \mathbb{R}^{m}$ are assumed to be continuous for all $x \in \mathbb{R}^{n}$.

A direct consequence of the continuity assumption is that for each scenario $\xi \in \mathcal{U}$ the outcome set is compact, which is the image of the feasible set,

$$
\mathcal{Y}(\xi):=\left\{y \in \mathbb{R}^{p} \mid \exists x \in X(\xi): y=f(x, \xi)\right\}=f(X(\xi), \xi) .
$$

For each scenario $\xi \in \mathcal{U}$, the Pareto set and the efficient set are denoted as $\mathcal{Y}_{P}(\xi)$ and $X_{E}(\xi)$ respectively.

As a concept of robustness to address the uncertain multiobjective problem $\{\mathcal{P}(\xi)\}_{\xi \in \mathcal{U}}$, point-based robust efficiency is applied, which leads to a robust counterpart that is an MOP and which is widely studied in the literature, see Section 3.4.1. The robust counterpart of $\{\mathcal{P}(\xi)\}_{\xi \in \mathcal{U}}$ is hence given by (3.13), where the objective function is defined by $(3.12)$.

The robust feasible set $X^{\mathrm{RC}}$ is compact since it is the intersection of the feasible sets of the scenarios $\mathcal{Y}(\xi)$ by Definition 3.1.1. The robust outcome set, which is the outcome set of the robust feasible set $X^{\mathrm{RC}}$, is denoted as $\mathcal{Y}^{\mathrm{RC}}:=f^{\mathrm{RC}}\left(X^{\mathrm{RC}}\right)$.

The robust counterpart RC is a deterministic MOP and the robust Pareto set, that is the Pareto set of the robust counterpart, and the robust efficient set are denoted as 
$\mathcal{Y}_{P}^{\mathrm{RC}}$ and $X_{E}^{\mathrm{RC}}$, respectively.

As a first result, a basic geometric connection between the outcome sets of the scenarios and the robust outcome set is presented. In (Krüger, Schöbel, and Wiecek 2017, Prop. 4) it is shown that the robust outcome set is contained in the outcome set of each scenario plus the Pareto cone. This directly implies a geometric relation between the Pareto sets of the scenarios and the robust Pareto set. By (Krüger, Schöbel, and Wiecek 2017, Cor. 5), for each robust Pareto point and for each scenario, there exists a Pareto point of that scenario that dominates the robust Pareto point.

Point-based robust efficiency is a conservative robustness concept, since it favors solutions that are best in the worst-case. It is therefore of interest to compare this conservative approach with the optimistic approach of choosing an optimal solution to some arbitrary scenario $\xi \in \mathcal{U}$. To this end, the concept of the robustness gap for singleobjective robust optimization has been developed in Ben-Tal and Nemirovski 1998, 1999, see Definition 3.1.2 in Section 3.1. The single-objective robustness gap measures the distance between the robust optimal objective value and the optimal objective values of the scenarios. In multiobjective optimization the Pareto set takes the place of the optimal objective value. The multiobjective robustness gap is therefore defined as the minimal distance between the robust Pareto set $\mathcal{Y}_{P}^{\mathrm{RC}}$ and the Pareto sets of the scenarios $\mathcal{Y}_{P}(\xi)$, $\xi \in \mathcal{U}$.

Definition 4.2.1 (Krüger, Schöbel, and Wiecek 2017, Def. 8). For each $\xi \in \mathcal{U}$ let

$$
\vartheta(\xi):=\operatorname{dist}\left(\mathcal{Y}_{P}^{\mathrm{RC}}, \mathcal{Y}_{P}(\xi)\right)=\inf _{z \in \mathcal{Y}_{P}^{\mathrm{RC}}} \inf _{y \in \mathcal{Y}_{P}(\xi)}\|z-y\|
$$

and define the multiobjective robustness gap as

$$
\vartheta:=\inf _{\xi \in \mathcal{U}} \vartheta(\xi)=\inf _{\xi \in \mathcal{U}} \operatorname{dist}\left(\mathcal{Y}_{P}^{\mathrm{RC}}, \mathcal{Y}_{P}(\xi)\right)
$$

The single objective robustness gap and the multiobjective robustness gap are illustrated in (Krüger, Schöbel, and Wiecek 2017, Ex. 7) and (Krüger, Schöbel, and Wiecek 2017, Ex. 9).

The multiobjective robustness gap is hard to compute, because it requires minimizing the distance between Pareto sets, which are generally nonconvex even for convex MOPs. For convex MOPs, a lower and an upper bound on the robustness gap are developed that are easier to calculate then the robustness gap itself. For the remainder of the publication the following Convexity Assumption is assumed to hold: It is required that $f(\cdot, \xi): \mathbb{R}^{n} \rightarrow \mathbb{R}^{p}$ be $\mathbb{R}_{\geqq}^{p}$-convex and $F(\cdot, \xi): \mathbb{R}^{n} \rightarrow \mathbb{R}^{m}$ be $\mathbb{R}_{\geqq}^{m}$-concave for all $\xi \in \mathcal{U}$, which implies that each instance of $\{\mathcal{P}(\xi)\}_{\xi \in \mathcal{U}}$ is a convex MOP.

Because the problems $\mathcal{P}(\xi)$ for all $\xi \in \mathcal{U}$, and the robust counterpart RC are convex MOPs, the efficient solutions to each of these MOPs can be determined by solving the single-objective problems that correspond to the weighted-sum scalarizations of the MOPs, see Proposition 3.2.5 in Section 3.2. 
Applying the weighted-sum scalarization to each instance of the problems $\{\mathcal{P}(\xi)\}_{\xi \in \mathcal{U}}$ and RC, we obtain an uncertain scalarized problem

$$
\left\{\begin{array}{lll}
(\mathcal{P}(\xi, \lambda)) \quad \min _{x} & \lambda^{T} f(x, \xi) \\
\text { s. t. } & x \in X(\xi)
\end{array}\right\}_{\xi \in \mathcal{U}},
$$

and the scalarized robust counterpart

$$
\begin{array}{lll}
(\mathrm{RC}(\lambda)) \quad \min _{x} & \lambda^{T} f^{\mathrm{RC}}(x) \\
\text { s. t. } & x \in X^{\mathrm{RC}},
\end{array}
$$

for each weight vector $\lambda \in \mathbb{R}_{\geq}^{p} \subseteq\left(\mathbb{R}^{p},\|\cdot\|_{*}\right)$. The weights $\lambda$ are here the linear functionals that are the elements of the dual cone to the Pareto cone $\mathbb{R}_{\geq}^{p} \subseteq\left(\mathbb{R}^{p},\|\cdot\|\right)$.

These are single objective problems and applying the definition of the single-objective robustness gap, see Definition 3.1.2 in Section 3.1, yields

$$
\min _{x \in X^{\mathrm{RC}}} \lambda^{T} f^{\mathrm{RC}}(x)-\sup _{\xi \in \mathcal{U}} \min _{x \in X(\xi)} \lambda^{T} f(x, \xi)=\inf _{\xi \in \mathcal{U}}\left(\min _{z \in \mathcal{Y}^{\mathrm{RC}}} \lambda^{T} z-\min _{y \in \mathcal{Y}(\xi)} \lambda^{T} y\right) .
$$

This is used to define the lower and the upper robustness bounds for the robustness gap $\vartheta$.

Definition 4.2.2 (Krüger, Schöbel, and Wiecek 2017, Def. 10). For each $\xi \in \mathcal{U}$ and for each $\lambda \in \mathbb{R}_{\geqq}^{p}$, we define

$$
\Delta(\xi, \lambda):=\min _{z \in \mathcal{Y}^{\mathrm{RC}}} \lambda^{T} z-\min _{y \in \mathcal{Y}(\xi)} \lambda^{T} y
$$

Furthermore, we define the lower robustness bound and upper robustness bound as

$$
\Delta^{L}:=\inf _{\xi \in \mathcal{U}} \min _{\substack{\lambda \in \mathbb{R}_{\geq}^{p},\|\lambda\|_{*}=1}} \Delta(\xi, \lambda) \quad \text { and } \quad \Delta^{U}:=\inf _{\xi \in \mathcal{U}} \max _{\substack{\lambda \in \mathbb{R}_{\geq}^{p},\|\lambda\|_{*}=1}} \Delta(\xi, \lambda) .
$$

It is then stated that the single-objective robustness gap coincides with the lower and the upper robustness bound in case of $p=1$, which further motivates the definition of the lower robustness bound $\Delta^{L}$ and upper robustness bound $\Delta^{U}$. Before it is proven that $\Delta^{L}$ and $\Delta^{U}$ are indeed a lower and an upper bound of the robustness gap $\vartheta$, it is shown that the lower and upper bound are nonnegative in (Krüger, Schöbel, and Wiecek 2017, Prop. 11) and well-defined in (Krüger, Schöbel, and Wiecek 2017, Prop. 13). The lower and the upper robustness bound $\Delta^{L}$ and $\Delta^{U}$ are illustrated by continuing the example on the robustness gap, see (Krüger, Schöbel, and Wiecek 2017, Ex. 12).

It is then proven that $\Delta^{L}$ is a lower bound of the robustness gap $\vartheta$.

Theorem 4.2.3 (Krüger, Schöbel, and Wiecek 2017, Th. 14). The lower robustness bound $\Delta^{L}$ is a lower bound for the robustness gap $\vartheta$, i.e.,

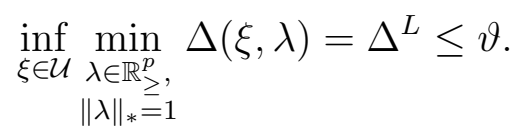


Second it is shown that the upper robustness bound $\Delta^{U}$ is indeed an upper bound of $\vartheta$ under the following condition and three significant cases are presented in which it is satisfied.

Condition A. Let the primal space be $\left(\mathbb{R}^{p},\|\cdot\|\right)$ with the dual space $\left(\mathbb{R}^{p},\|\cdot\|_{*}\right)$. We say that Condition $A$ holds for a scenario $\bar{\xi} \in \mathcal{U}$ if there exist $\bar{z} \in \mathcal{Y}_{P}^{\mathrm{RC}}, \bar{y} \in \mathcal{Y}_{P}(\bar{\xi})$ and $\bar{\lambda} \in\left(\mathbb{R}^{p},\|\cdot\|_{*}\right)$ such that

(i) $\bar{\kappa}:=\bar{z}-\bar{y} \in \mathbb{R}_{\geq}^{p} \subseteq\left(\mathbb{R}^{p},\|\cdot\|\right)$ and $\bar{\lambda} \in \mathbb{R}_{\geq}^{p} \subseteq\left(\mathbb{R}^{p},\|\cdot\|_{*}\right)$

(ii) $\bar{\lambda} \in \arg \max _{\lambda \in \mathbb{R}_{>}^{p},\|\lambda\|_{*}=1} \bar{\kappa}^{T} \lambda$

(iii) $\bar{z} \in \arg \min _{z \in \mathcal{Y}^{\mathrm{RC}}} \bar{\lambda}^{T} z$.

The condition is illustrated for the ongoing example in (Krüger, Schöbel, and Wiecek 2017, Fig. 3), and the main result about the upper bound on the robustness gap is given as follows.

Theorem 4.2.4 (Krüger, Schöbel, and Wiecek 2017, Th. 16). If Condition A is satisfied for all $\xi \in \mathcal{U}$ with $\mathcal{Y}_{P}(\xi) \cap \mathcal{Y}_{P}^{\mathrm{RC}}=\emptyset$ then

$$
\vartheta \leq \Delta^{U}
$$

Moreover, it is remarked in (Krüger, Schöbel, and Wiecek 2017, Rm. 17) that for every scenario $\bar{\xi}$ in which the robustness gap is attained and for which Condition $\mathrm{A}$ is satisfied there exists a weight $\bar{\lambda}$ such that the robustness gap corresponds to $\Delta(\bar{\xi}, \bar{\lambda})$ and then the robustness gap can directly be calculated.

Three cases in which Condition $\mathrm{A}$ is satisfied are next investigated in which the primal norm is a $\mathfrak{p}$ norm with $1 \leq \mathfrak{p} \leq \infty$. The three cases differ with respect to the dimension of the objective space and the considered norm. In the first investigated case in (Krüger, Schöbel, and Wiecek 2017, Lem. 18), the dimension $p$ of the primal space is arbitrary and the primal norm is considered as the $\mathfrak{p}$-norm $\|\cdot\|_{1}$.

In the second investigated case in (Krüger, Schöbel, and Wiecek 2017, Lem. 19), the dimension of the primal space is again arbitrary. It is proven that Condition $\mathrm{A}$ is satisfied if the primal norm is a $\mathfrak{p}$-norm with $1<\mathfrak{p} \leq \infty$ under an additional assumption on the ideal point $z_{\mathrm{RC}}^{I}$ of the robust counterpart that is $z_{\mathrm{RC}}^{I} \in \mathcal{Y}(\bar{\xi})+\mathbb{R}_{\geqq}^{p}$, for a scenario $\bar{\xi}$ with $\mathcal{Y}_{P}(\bar{\xi}) \cap \mathcal{Y}_{P}^{\mathrm{RC}}=\emptyset$.

In the third investigated case in (Krüger, Schöbel, and Wiecek 2017, Lem. 20), it is shown that Condition $\mathrm{A}$ is valid for every $\mathfrak{p}$-norm with $1 \leq \mathfrak{p} \leq \infty$ without any further assumptions if the dimension of the primal space is $p=2$, i.e., the uncertain problem $\{\mathcal{P}(\xi)\}_{\xi \in \mathcal{U}}$ is biobjective.

In addition to the three investigated cases, two sufficient conditions for Condition A are mentioned that involve normal cones as defined in Rockafellar 2015 and restricted normal cones as defined in Bauschke, Luke, Phan, and Wang 2013. 
A major result on the single-objective robustness gap is then extended to the multiobjective case for convex MOPs. This result, see Theorem 3.1.3 in Section 3.1, states that the robustness gap is zero under certain assumptions including constraint-wise uncertainty. Assumption 3.1.1 together with the continuity assumption and the convexity assumption corresponds to the assumptions of (Ben-Tal and Nemirovski 1998, Th. 2.1), which is the original source of the result. The same assumptions are used for the multiobjective case and they are extended by the assumption objective-wise uncertainty on the objective function, see Section 3.4.3.

As a preparation for the result on the zero robustness gap it is first shown that, under these assumptions, for every robust Pareto point $z \in \mathcal{Y}_{P}^{\mathrm{RC}}$ and every neighborhood of this point there exists a scenario $\xi$ with a Pareto point $y \in \mathcal{Y}_{P}(\xi)$ that falls within this neighborhood.

This result is then used to show that the assumptions that are sufficient for the zero robustness gap in the single-objective case combined with objective-wise uncertainty are sufficient for the zero robustness gap in the multiobjective case.

Theorem 4.2.5 (Krüger, Schöbel, and Wiecek 2017, Th. 23). Let the Continuity Assumption, the Convexity Assumption and Assumption 3.1.1 hold and let the uncertainty set $\mathcal{U}$ be constraint-wise and objective-wise. Then

(a) The robust counterpart $\mathrm{RC}$ is feasible if and only if all instances of $\{\mathcal{P}(\xi)\}_{\xi \in \mathcal{U}}$ are feasible.

(b) If $f^{\mathrm{RC}}$ is given as in (3.12) then the robustness gap vanishes, i.e., $\vartheta=0$.

In the final part of the publication, uncertain linear MOPs (MOLPs) are considered and the two types of uncertainty decision uncertainty and parameter uncertainty are distinguished.

Decision uncertainty in linear multi-objective problems is investigated first and it is modeled within the framework proposed in Eichfelder, Krüger, and Schöbel 2017, which is summarized in Section 4.1. Also the corresponding concepts of decision robust feasibility and decision robust efficiency are used. In this setting, it is assumed that each decision vector $x$ is realized within the set $x+Z$, where the perturbation set $Z$ is a compact and convex subset of $\mathbb{R}^{p}$. Note that, in order to distinguish decision and parameter uncertainty in this summary, the perturbation set is denoted as $Z$ rather than $\mathcal{U}$. An MOLP with decision uncertainty is then given as

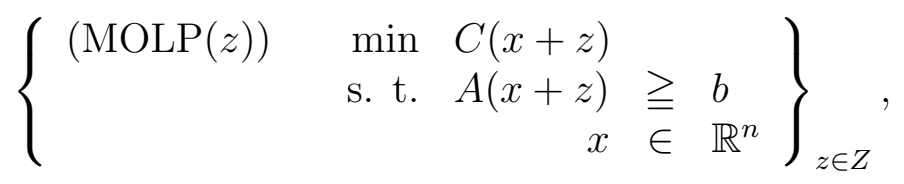

where the problem data satisfies $(C, A, b) \in \mathbb{R}^{p \times n} \times \mathbb{R}^{m \times n} \times \mathbb{R}^{m}$. In this setting, the decision robust counterpart is a set-valued optimization problem by definition. However, the objective function of the uncertain problem $\{\operatorname{MOLP}(z)\}_{z \in Z}$ is linear and Theorem 4.1.4 applies. The decision robust counterpart of $\{\operatorname{MOLP}(z)\}_{z \in Z}$ can hence be considered as a semi-infinite MOLP and the decision uncertainty is only contained in its constraints. 
According to a result from the literature, see (Ben-Tal, Ghaoui, and Nemirovski 2009, Th. 1.3.4), the decision robust feasible set $X^{\mathrm{RC}}$ can equivalently be represented by finitely many linear inequalities.

The set $X^{\mathrm{RC}}$ can therefore be considered as a polyhedral set, which is used in (Krüger, Schöbel, and Wiecek 2017, Prop. 25) to derive a sufficient condition for the zero robustness gap in case of MOLPs with decision uncertainty. It is shown that the robustness gap is zero if there exists a decision robust efficient $p-1$-dimensional face of the polyhedral set $X^{\mathrm{RC}}$.

Moreover, a single-objective optimization problem whose optimal objective value corresponds to the lower bound $\Delta^{L}$ of the gap $\vartheta$ is given. This problem is obtained by combining the polyhedral description of $X^{\mathrm{RC}}$ with the definition of the lower bound $\Delta^{L}$ and by applying linear programming duality to that part of $\Delta^{L}$ that corresponds to the robust optimal objective value of the scalarized problem. The problem is a DC optimization problem if the objective matrix $C \in \mathbb{R}^{p \times n}$ is positive semidefinite, if the perturbation set $Z$ is a convex polytope and if the primal norm $\|\cdot\|$ is a block norm, i.e., the unit ball of the primal norm is a polyhedral set.

For the upper bound $\Delta^{U}$, an optimization problem whose optimal objective value corresponds to $\Delta^{U}$ is derived in a similar way. The polyhedral formulation of $X^{\mathrm{RC}}$ is combined with Definition 4.2.2 and applying linear programming duality yields a biobjective problem that has linear constraints and a quadratic objective function on the lower level.

For MOLPs with parameter uncertainty, single-objective optimization problems whose optimal objective values correspond to the lower and upper bound are also given. In case of parameter uncertainty the objective function $C \in \mathbb{R}^{p \times n}$ and the linear constraints $A \in \mathbb{R}^{m \times n}, b \in \mathbb{R}^{m}$ are considered uncertain, and the scenarios of the uncertainty set are denoted as $\xi=(C, A, b) \in \mathcal{U}$. An MOLP with parameter uncertainty is considered as

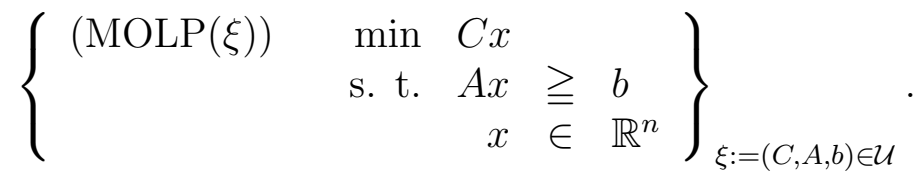

It is remarked that this setting is not only suitable to address MOLPs with parameter uncertainty but also MOLPs with both decision and parameter uncertainty, because as we have seen previously in (Eichfelder, Krüger, and Schöbel 2017, Prop. 21), every MOLP with decision uncertainty can be formulated as an MOLP with parameter uncertainty. In this publication, MOLPs with parameter uncertainty are treated as a special case of $\{\mathcal{P}(\xi)\}_{\xi \in \mathcal{U}}$. Therefore, the concept of robust feasibility applies, see Definition 3.1.1 and the robust counterpart of $\{\operatorname{MOLP}(\xi)\}_{\xi \in \mathcal{U}}$ is a semi-infinite MOLP.

The robust counterpart $\mathrm{RC}^{\mathrm{MOLP}}$ is reformulated such that only the constraints are affected by parameter uncertainty, which is frequently done in the literature, see, e.g., Ben-Tal, Ghaoui, and Nemirovski 2009 for single objective reformulations and Fliege and Werner 2014 and Goberna, Jeyakumar, Li, and Vicente-Pérez 2014 for multiobjective reformulations. Because the problem $\mathrm{RC}^{\mathrm{MOLP}}$ then is a semi-infinite MOLP that is 
affected by uncertainty only in the constraints, a result from single-objective optimization can be applied. According to (Ben-Tal, Ghaoui, and Nemirovski 2009, Cor. 1.3.5), the constraints of a semi-infinite MOLP can be represented by a finite system of linear inequalities if the uncertainty set $\mathcal{U}$ is a polyhedral set. The robust counterpart can therefore be considered as an MOLP.

A single objective problem whose optimal objective value corresponds to the lower bound $\Delta^{L}$ of the gap $\vartheta$ is it then developed analogously to the previous case of decision uncertainty in MOLPs.

The description of $\mathrm{RC}^{\mathrm{MOLP}}$ as an MOLP is combined with the definition of $\Delta^{L}$, and, applying linear programming duality yields a DC optimization problem if the linear objective function of the robust counterpart is positive semidefinite and if the dual norm can be formulated as linear constraints, which is the case for block norms.

For the upper bound $\Delta^{U}$, an optimization problem whose optimal objective value corresponds to $\Delta^{U}$ is derived in a similar way. The polyhedral formulation of $\mathrm{RC}^{\mathrm{MOLP}}$ is combined with Definition 4.2.2 and applying linear programming duality yields a biobjective problem that has linear constraints and a quadratic objective function on the lower level.

In summary, a concept for a multiobjective robustness gap has been developed that applies to minmax robust counterparts that are MOPs. For convex problems, a lower and an upper bound for the gap are given. For linear problems, the lower and upper bounds can be calculated by solving the presented single-objective problems.

\section{Own Contribution}

The central idea how to define the multiobjective robustness gap and suitable lower and upper bounds was developed together by all coauthors. The proof of the lower bound and the development of the single-objective problems that yield the bounds in the linear case are also joint work. The proofs of the upper bound and of the zero robustness gap under certain conditions, and the ongoing example were mostly contributed by the author of this thesis with the help and advice of the coauthors. The structure of this publication is joint work of all coauthors and the largest part of its writing was done by the author of this thesis. 


\subsection{An Application of Robust Multiobjective Optimization in Agriculture}

The publications Krüger, Castellani, Geldermann, and Schöbel 2018 and Krüger 2018 are summarized next. The former is joint work with Francesco Castellani, Prof. Dr. Jutta Geldermann and Prof. Dr. Anita Schöbel and the latter was written by the author of this thesis.

The research presented in this section is the result of an interdisciplinary cooperation within the research training group RTG 1703 "Resource Efficiency in Corporate Networks". An uncertain biobjective optimization problem that is relevant for the commercial cultivation of potted plants is investigated and it is called the peat-and-pots problem. This work is composed of two parts: a practical and a theoretical study of the problem.

The practical study of the peat-and-pots problem is motivated by an application in an Italian plant nursery that is described in detail in Krüger, Castellani, Geldermann, and Schöbel 2018. The decision problem is introduced such that it reflects the information gained through this application. The resulting decision problem is addressed in two ways. First, it is considered as a deterministic problem and the efficient solutions are calculated. Second, parameter uncertainty and decision uncertainty are taken into account and the decision robust efficient solutions are calculated. The two solution approaches are then compared and it is found that the robust solutions are preferable over the deterministic solutions.

The theoretical study of the peat-and-pots problem that is given in Krüger 2018 aims to determine the efficient solutions to the deterministic peat-and-pots problem and the decision robust efficient solutions to the uncertain peat-and-pots problem. The presented solution approaches are suitable to solve a slightly generalized version of the problem that is considered in the practical study Krüger, Castellani, Geldermann, and Schöbel 2018 and hence, this study provides the theoretical background for the aforementioned publication. In the last part of this publication, two extensions of the problem are discussed and suitable approaches to determine deterministic and robust solutions are given.

The practical viewpoint on the problem as contained in Krüger, Castellani, Geldermann, and Schöbel 2018 is summarized in Section 4.3.1 and the theoretical background of the problem as presented in Krüger 2018 is summarized in Section 4.3.2.

\subsubsection{Peat and Pots: An Application of Robust Multiobjective Optimization to a Mixing Problem in Agriculture}

In the following, the publication Krüger, Castellani, Geldermann, and Schöbel 2018 is summarized, which can be considered as a practical study on the peat-and-pots problem.

The aim of the peat-and-pots problem is to choose a mixture of growth substrate and a 
type of planter pot for the commercial cultivation of potted plants such that a monetary and an environmental objective are optimized. The peat-and-pots problem is therefore a biobjective optimization problem. While the consideration of a monetary objective is typical for enterprises such as plant nurseries, the consideration of the environmental impact as a second objective is motivated by recent research on the greenhouse gas emissions of cultivation processes in the horticulture sector as described in detail in Section 2 of this publication. In that section, also the advantages and disadvantages of different types of planter pots and growth media are discussed. The planter pots that can be chosen are plastic pots or biodegradable pots (bio-pots) and the growth medium can been chosen as a mixture of peat and compost. The properties of these input materials that are relevant for the biobjective problem are their costs and their greenhouse gas emissions.

The classical and widely used option is a plastic pot filled with $100 \%$ peat. Plastic pots are cheaper than bio-pots. However, plastic pots are generally landfilled after use, while bio-pots can be put into the soil along with the plant, and hence, the former cause a larger quantity of greenhouse gas emissions than the latter.

The growing medium peat is traditionally used because of its agronomic properties that lead to high quality plants. Peat is more expensive than its substitute compost, which is a byproduct from other industries such as the olive oil industry. Moreover, the harvesting of peat causes significant $\mathrm{CO}_{2}$ emissions because peatlands are important carbon sinks. Peatlands also provide important living spaces for various plant and animal species and are vital for biodiversity and ecosystem services. Replacing peat with compost therefore reduces costs and greenhouse gas emissions at the same time.

The monetary objective function of the peat-and-pots problem are the additional costs $(\mathrm{adC})$ that are the difference between the decision relevant costs of the input materials and the selling price of a potted plant. The environmental objective function is the global warming potential (GWP) of the decision relevant input materials.

Following on this motivation and introduction to the peat-and-pots problem, an application is presented in detail in Section 3 of this publication. A case study on the cultivation of the species Photinia $x$ fraseri by an Italian plant nursery is described and the data collection process for the costs and emissions of the input materials plastic pots, bio-pots, peat and compost is reported.

The peat-and-pots problem is then modeled as a deterministic biobjective optimization problem that reflects the case study. It is assumed that a plant nursery uses the same planter pot and the same mixture of growth medium for all potted plants of the same species, i.e., one potted plant represents all plants of the species Photinia and only one plant is considered in the decision problem. 
The (deterministic) peat-and-pots problem is introduced as

$$
\begin{array}{ccc}
\min & \left(\begin{array}{c}
\operatorname{adC}(\gamma, \beta) \\
G W P(\gamma, \beta)
\end{array}\right) \\
\text { s.t. } & Q(\gamma, \beta) \geq c \\
& \quad \geq \quad \in[0,1] \\
& \beta & \in\{0,1\} .
\end{array}
$$

There are two decision variables in the peat-and-pots problem:

- The share of compost $\gamma \in[0,1]$ used to replace peat.

- The type of pots $\beta \in\{0,1\}$ to use, where $\beta=\left\{\begin{array}{ll}1 & \text { if bio-pot chosen } \\ 0 & \text { if plastic pot chosen }\end{array}\right.$.

Due to the first constraint, the quality of the potted plant that is associated with a solution has to be high enough for selling. There is a finite set of quality levels and the quality function $Q$ maps each solution to the quality level that reflects the plant's aesthetic and agronomic properties. For the species Photinia, the minimum salable quality level is 3 .

To complete the description of the deterministic peat-and-pots problem, it remains to describe the two objective functions in detail. The monetary objective that is the additional costs (adC) is considered as the difference between the costs of the input materials and the selling price of the potted plant. These two parts of the adC function are next introduced separately.

The cost function costs is defined as the sum of the decision relevant input materials that is the planter pot and the growth medium. The function costs is therefore defined as costs: $[0,1] \times\{0,1\} \rightarrow \mathbb{R}$ with

$$
\operatorname{costs}(\gamma, \beta)=c^{\text {peat }}+c^{\text {plas }}+\gamma \cdot\left(c^{\text {comp }}-c^{\text {peat }}\right)+\beta \cdot\left(c^{\text {bio }}-c^{\text {plas }}\right),
$$

where the parameters $c^{\text {peat }}, c^{c o m p}, c^{\text {plas }}, c^{\text {bio }}$ denote the costs of one functional unit of peat or compost and the cost of a plastic pot or a bio pot, respectively.

The selling price of a solution is determined by combining two functions: the quality function $Q$ that maps each solution to the quality level of the potted plant and the function price that assigns each quality level to a selling price.

The quality function is a piecewise constant function on the share of compost for each pot $\beta \in\{0,1\}$. A plant of the species Photinia can attain any quality level in $\{1, \ldots, 5\}$ and the quality function $Q$ for this species is illustrated in Figure 4.1, which corresponds to (Krüger, Castellani, Geldermann, and Schöbel 2018, Fig. 1). It is assumed that the quality level of a plant raised in a bio-pot is higher or equal than the quality of a plant raised in a plastic pot on a identical mixture of growth substrate under equivalent agricultural conditions.

Without loss of generality, it can be assumed that the quality levels are defined such that a higher quality always implies a higher selling price, i.e., the selling price function 


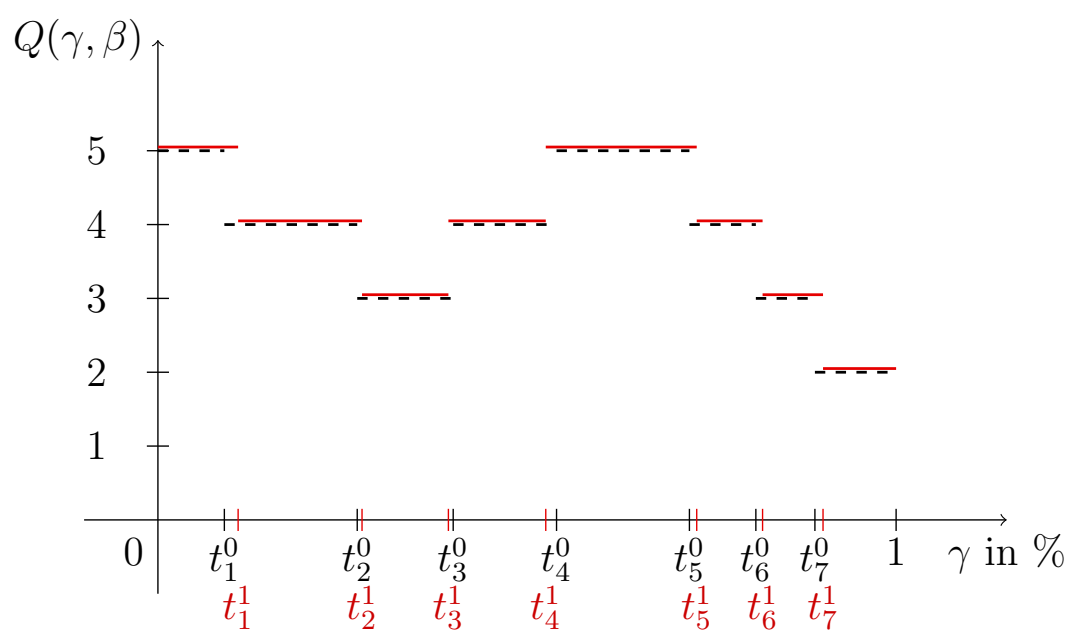

Figure 4.1.: Quality function for the species Photinia. Dashed lines indicate a plastic pot $(\beta=0)$ and straight lines indicate a biodegradable pot $(\beta=1)$.

price is monotonically increasing on the quality levels. The selling price of each quality level is then given by the monotonically increasing price function

$$
\text { price }:\{1, \ldots, 5\} \rightarrow \mathbb{R}
$$

Combining the cost function costs with the quality function $Q$ and the related price function price yields the monetary objective function

$$
\operatorname{adC}(\gamma, \beta):=\operatorname{costs}(\gamma, \beta)-\operatorname{price}(Q(\gamma, \beta)) .
$$

The second objective function is the global warming potential GWP that is measured in terms of greenhouse gas emissions. The greenhouse gas emissions of the input materials are the linear combination of the emissions of the individual input materials and thus $G W P$ is defined as

$$
G W P(\gamma, \beta)=e m^{\text {peat }}+e m^{\text {plas }}+\gamma \cdot\left(e m^{\text {comp }}-e m^{\text {peat }}\right)+\beta \cdot\left(e m^{\text {bio }}-e m^{\text {plas }}\right),
$$

where the parameters $\mathrm{em}^{\text {peat }}, \mathrm{em}^{\mathrm{comp}}, \mathrm{em}^{\text {plas }}, \mathrm{em}^{\text {bio }}$ denote the greenhouse gas emissions of one functional unit of peat or compost and the emissions of a plastic pot or a bio pot, respectively.

After the deterministic peat-and-pots problem has been introduced, it is addressed in two ways: It is first considered as a deterministic biobjective problem and a solution approach to determine its (Pareto-)efficient solutions is discussed. Second, it is recognized that the problem is affected by decision and parameter uncertainty and a solution approach to determine the decision robust efficient solutions is applied.

The idea of the deterministic appraoch is to solve the problem in two steps: first, to solve it once for each value of the pot variable $\beta \in\{0,1\}$ and second, to compare the 
resulting solution sets. For each fixed $\beta$, the efficient solutions can be computed by an analytical description that is developed in Krüger 2018. For the species Photinia in the case study that is described in Section 3 of this publication, this solution approach can be described as follows: Due to the values of the cost and emission parameters, it is observed that the objective functions adC and $G W P$ are both monotonically strictly decreasing on each of the constant intervals of the quality function and hence, only the endpoints of these intervals can be efficient solutions. Infeasible endpoints of the intervals are excluded, which means that the corresponding quality level of the remaining solutions is at least 3. This yields a finite candidate set and from this set the largest solutions are iteratively selected such that each solution is of a higher quality than the previously selected solution.

The uncertainty in the peat-and-pots problem and an approach to determine the decision robust efficient solutions is then described in Section 5 of the publication. The cost and emission parameters of the problem are affected by parameter uncertainty, due to fluctuations during the processing of the raw materials, for example fluctuations of the electricity consumption. According to the data collection as described in Section 3 of the publication, every parameter is known to fall within a given interval of values rather than being known as an exact number. By definition of the objective functions, the cost parameters only contribute to the first objective function adC and the emission parameters only contribute to the second objective function $G W P$. Therefore, the parameter uncertainty is objective-wise and, by Section 3.4.3, the two minmax robustness concepts set-based robust efficiency and point-based robust efficiency coincide. The robust efficient solutions can hence be determined by replacing each parameter with the maximum value of its interval and by solving the resulting deterministic biobjective problem.

Thus, it remains to address the decision uncertainty in the peat-and-pots problem. The pot variable $\beta$ can naturally be implemented exactly, but the decision variable $\gamma$ that denotes the share of compost in the growth medium is affected by decision uncertainty, since the growth medium is prepared and mixed in big heaps. The plant nursery provides minimum perturbation $z^{\text {min }}$ and a maximum perturbation $z^{\max }$ for the share of compost. These minimum and maximum values are based on the plant nursery's experience and the precision of the mixing process. The decision variable $\gamma$ is implemented as an element of a perturbation set $Z(\gamma)$ that is an interval. The perturbation sets of all solutions are bounded by the minimum and maximum perturbation $z^{\text {min }}$ and $z^{\text {max }}$, but there are solutions that have individual smaller perturbation sets. This is because solutions can never be realized as less than $0 \%$ or more than $100 \%$ of compost. Hence, the perturbation sets of solutions near 0 or near 1 are smaller than the interval $\left[z^{\min }, z^{\max }\right]$ and for each solution $\gamma \in(0,1)$, the perturbation set is therefore given as

$$
Z(\gamma):=\left[\max \left\{z^{\min },-\gamma\right\}, \min \left\{z^{\max }, 1-\gamma\right\}\right]
$$

The solutions $\gamma \in\{0,1\}$ are exceptions that are realized exactly, because no mixing is required. Decision robust efficiency is then considered as the minmax robustness concept to address this uncertainty. Even though this robustness concept is introduced in Section 4.1 for the case that the perturbation set is constant for all solutions, it is applied 
here to the case of individual perturbation set. This slight extension of decision robust efficiency is also mentioned in the conclusions of the original publication Eichfelder, Krüger, and Schöbel 2017.

In order to determine the decision robust efficient solutions, the suitable solution approach that is presented in Krüger 2018 is applied. Similar to the previous deterministic approach, the problem is solved in two steps: first, it is solved once for each value of the pot variable $\beta \in\{0,1\}$ and second, the resulting sets of solutions are compared. For each fixed $\beta$, the decision robust efficient solutions can be computed by an analytical description that is given in Krüger 2018.

The solution approaches for the deterministic and the robust efficient solutions are similar in the way that the problem is solved for each $\beta$ by first constructing a finite candidate set. However, there are two major differences between them: First, the endpoints of the intervals of the quality function are considered in the deterministic approach, whereas, in the robust approach, a safety margin is kept towards the right hand interval bounds. Second, the resulting finite set of solutions is compared with respect to their quality in the former and it is compared with respect to the minimum quality of the realizations in the latter.

In Section 6 of this publication, these solution approaches are applied to solve the peatand-pots problem for the case study of the species Photinia and the data from Section 3 is used to determine the deterministic and the decision robust efficient solutions.

Because the peat-and-pots problem is investigated as a deterministic and as an uncertain problem, two sets of data are provided. The first set of the problem parameters for the costs and emissions contains the average values and the second set includes the minimal and maximal values that the parameters can attain, see (Krüger, Castellani, Geldermann, and Schöbel 2018, Tab. 1).

It is found that there are four distinct deterministic efficient solutions and four distinct decision robust efficient solutions. In both cases, deterministic and robust, only one solution involves a plastic pot while the other three solutions involve a biodegradable pot.

It is observed that in each case the solution with a plastic pot performs best with respect to the monetary objective function adC due to the price difference of the two pots. However, comparing the solution with the plastic pot to the efficient solution with a bio-plot that is smallest in the share of compost $\gamma$, the former solution is only slightly better in the first objective function adC while the latter it's significantly better in the second objective function $G W P$. In the uncertain case, the only decision robust efficient solution with a plastic pot is the traditional solution involving $100 \%$ peat. It is therefore concluded as a rule of thumb for the species Photinia that, in case of decision uncertainty, a substitution of peat with compost must be combined with replacing the plastic pots by biodegradable pots.

The deterministic and robust efficient solutions of the case study are then compared. It is important to note that the sets of deterministic efficient solutions and of decision robust efficient solutions in the case study are disjoint. Evaluating the four deterministic efficient solutions shows that two of the four solutions are not decision robust efficient, 
i.e., they have realizations that do not yield the minimum required quality. The remaining two deterministic efficient solutions are each dominated by one or more of the decision robust efficient solutions, which shows that the latter solutions are preferable over the former. In summary, it is concluded that (decision) robust efficiency is a concept that is worth considering to address the peat-and-pots problem with decision uncertainty.

\subsubsection{Peat and Pots: Analysis of Robust Solutions for a Biobjective Problem in Agriculture}

The publication Krüger 2018 that can be considered as a theoretical study of the peatand-pots problem is summarized next.

This publication provides the theoretical background for the solution approaches to the peat-and-pots problem that are applied in the case study in Krüger, Castellani, Geldermann, and Schöbel 2018. The peat-and-pots problem is considered first as a deterministic problem and a solution approach to determine the efficient solutions is developed. Second, the parameter and decision uncertainty in the problem are taken into account and a solution approach to determine the decision robust efficient solutions is given.

The motivation and description of the peat-and-pots problem correspond in large parts to those in the previously summarized publication Krüger, Castellani, Geldermann, and Schöbel 2018. Therefore, only those parts of the problem description that are not contained in the former publication are stated here.

In the Section 4.3.1, the problem is modeled in a way that reflects the specific setting of the case study. In this theoretical investigation of the peat-and-pots problem the problem is considered in a more general way. The problem investigated here extends the peat-and-pots problem in the previous section by two properties: First, the problem is modeled for arbitrary plant species and hence, the quality function is defined in a more general way. Second, the availability of compost is considered to be limited throughout this publication, since, in practice, compost occurs as a byproduct rather than being produced intentionally.

The peat-and-pots problem is given as follows:

$$
\begin{aligned}
& \min \left(\begin{array}{c}
\operatorname{adC}(\gamma, \beta) \\
G W P(\gamma, \beta)
\end{array}\right) \\
& \text { s.t. } Q(\gamma, \beta) \geq \alpha \\
& \gamma \leq v^{c o m p} \\
& \gamma \in[0,1] \\
& \beta \in\{0,1\} \text {. }
\end{aligned}
$$

The quality function is given as

$$
Q:[0,1] \times\{0,1\} \rightarrow\{1, \ldots, \nu\},
$$


where $\nu$ denotes the maximum quality of the considered species, and where $\{1, \ldots, \nu\}$ is the set of quality levels. The minimum quality that is salable depends on the species and it is denoted as $\alpha$. The quality function $Q$ is a piecewise constant function for each fixed planter pot $\beta \in\{0,1\}$. Its constant parts can be expressed by their boundary points

$$
0=t_{0}^{\beta}<t_{1}^{\beta}<t_{2}^{\beta}<\cdots<t_{\omega}^{\beta}=1
$$

where $\omega$ is the number of the quality function's constant parts. For every $1 \leq i \leq \omega$, there is $q_{i} \in\{1, \ldots, \nu\}$ such that $Q(\gamma, \beta)=q_{i}$ for all $\gamma \in\left(t_{i-1}^{\beta}, t_{i}^{\beta}\right)$. The quality level of each interval bound that belongs to two intervals is defined as the maximum quality of the two intervals, that is

$$
Q(\gamma, \beta)= \begin{cases}q_{i}, & \text { if } \gamma \in\left(t_{i-1}^{\beta}, t_{i}^{\beta}\right), \text { for } i=1, \ldots, \omega \\ \max \left\{q_{i}, q_{i+1}\right\} & \text { if } \gamma=t_{i}^{\beta} \text { for } i=1, \ldots, \omega\end{cases}
$$

The solutions $t_{0}^{\beta}=0$ and $t_{\omega}^{\beta}=1$ are boundary points for only one interval each and their quality is defined as $Q\left(t_{0}^{\beta}, \beta\right)=q_{1}$ and $Q\left(t_{\omega}^{\beta}, \beta\right)=q_{\omega}$, respectively. Therefore, the quality function $Q$ is not continuous but upper semicontinuous on the share of compost $\gamma \in[0,1]$. As in Section 4.3.1, it is assumed that the quality of a plant raised in a biodegradable pot is higher or equal than that of a plant raised in a plastic pot on an identical mixture of growth substrate.

As the second extension of the problem in Section 4.3.1, it is required that the share of compost $\gamma$ is smaller than the available quantity of compost $v^{\text {comp }}$ in the second constraint of $\mathcal{P}$, because it is assumed that the available quantity of compost is limited. In fact, $v^{\text {comp }}$ denotes the available quantity of compost per pot, since the functional unit of the problem is one plant as a representative of its species.

The aim of this publication is to find solution approaches for the deterministic and the uncertain peat-and-pots problem. In particular, it is intended to solve the case study in Krüger, Castellani, Geldermann, and Schöbel 2018. The data that characterizes the case study has three properties that must be taken into account in a suitable solution approach. The three properties are therefore stated as three assumptions. The first assumption, (Krüger 2018, Ass. 1) is that the cost of compost $c^{\text {comp }}$ is strictly smaller than the cost of peat $c^{\text {peat }}$. The second assumption, (Krüger 2018, Ass. 2) is that for each pair of solutions that yield distinct quality levels their cost difference is smaller than the difference of their selling prices. The third assumption, (Krüger 2018, Ass. 3) is that the $G W P$ of the traditional input materials plastic pots and peat is strictly larger than the $G W P$ of their alternatives composts and biodegradable pots, respectively.

If first and second assumptions are met, the objective function adC has two properties that lead to a simple structure of the solution set of the peat-and-pots problem in the subsequent results. In (Krüger 2018, Lem. 1) it is shown that adC is monotonically strictly decreasing on each of the constant parts of the quality function. In (Krüger 2018, Lem. 2) it is proven that an increase of quality always causes a decrease of the additional costs. 
If the third assumption is met, the second objective function $G W P$ is monotonically strongly decreasing as shown in (Krüger 2018, Lem. 3).

The peat-and-pots problem is next solved such that it reflects the properties of the case study, that is the three assumptions are taken into account.

In Section 3 of the publication, the peat-and-pots problem is addressed as a deterministic problem. The classical optimality concept Pareto efficiency, see Definition 3.2.2, is considered and the (deterministic) efficient solutions to the peat-and-pots problem are determined.

The presented solution approach for the peat-and-pots problem is based on the idea to solve the problem once for each value of the pot variable $\beta \in\{0,1\}$, followed by a pairwise comparison of the obtained solutions. The efficient solutions for each $\beta$ can be calculated with little computational effort, because an analytical description of the solutions is provided in the publication and the number of efficient solutions is small for each $\beta \in\{0,1\}$.

Three results on the deterministic efficient solutions to the peat-and-pots problem are given in the publication. It is first shown, that the weakly and the strictly efficient solutions of the peat-and-pots problem coincide for each $\beta \in\{0,1\}$ under the three assumptions that reflect observations on the problem data. This is stated in (Krüger 2018, Prop. 5). Hence, weak and strict (deterministic) efficiency do not need to be distinguished subsequently and it is sufficient to determine the efficient solutions for each $\beta \in\{0,1\}$.

The second and main result on the deterministic efficient solutions is their analytical description for each $\beta$.

Theorem 4.3.1 (Krüger 2018, Th. 6). For each fixed pot variable $\beta \in\{0,1\}$, the set

$$
\mathcal{D}(\beta):=\bigcup_{j=\nu_{\min }}^{\nu}\{\max \{\gamma \in \mathfrak{F}(\beta) \mid Q(\gamma, \beta) \geq j\}\},
$$

is the set of efficient solutions.

As a consequence of this theorem, the deterministic efficient solutions are a finite set for each $\beta \in\{0,1\}$. The maximum share of compost for each considered quality or higher exists, because the quality function $Q$ is upper semicontinuous. Furthermore, this maximum share of compost falls on a boundary point of a constant interval.

As the third and last result on the deterministic efficient solutions, it is stated that there always exists an efficient solution to the peat-and-pots problem, see (Krüger 2018, Cor. 7), which follows directly from the finiteness of the efficient set for each $\beta \in\{0,1\}$.

In Section 4, the presence of the parameter and decision uncertainty in the peat-andpots problem is recognized and motivated by the case study. Parameter uncertainty is discussed in Section 4.1. The economic objective adC is only affected by the cost parameters $(c)$ and the environmental objective $G W P$ is only affected the emission parameters $(\mathrm{em})$. Based on the data of the case study and on the background of the 
data collection described in Krüger, Castellani, Geldermann, and Schöbel 2018, it is assumed that the uncertainty set is a direct product of compact intervals, where each interval contains the values one parameter can attain.

$$
\mathcal{U}=\left\{(c, e m) \mid c^{\text {peat }} \in\left[\underline{c}^{\text {peat }}, \bar{c}^{\text {peat }}\right], \cdots, e m^{\text {bio }} \in\left[\underline{e m}^{b i o}, \overline{e m}^{b i o}\right]\right\} .
$$

The parameter uncertainty is therefore objective-wise and the two minmax robustness concepts set-based robust efficiency and point-based robust efficiency coincide, see Section 3.4. Because the uncertainty set is compact and because the decision variables are nonnegative, there exists a worst-case scenario $(\bar{c}, \overline{e m})$ that corresponds to the maximum value of each interval. To determine the minmax robust efficient solutions to the peat-and-pots problem with parameter uncertainty, it is therefore sufficient to solve the deterministic problem with respect to the worst-case scenario.

Decision uncertainty in the peat-and-pots problem is investigated in Section 4.2 of the publication. The planter pot is generally used as intended and only the decision variable $\gamma$ that represents the share of compost is affected by decision uncertainty, since the mixing of growth substrate is only performed roughly in plant nurseries. As described in Section 4.3.1, the perturbation set of a solution $\gamma$ is given as

$$
Z(\gamma):=\left[\max \left\{z^{\min },-\gamma\right\}, \min \left\{z^{\max }, 1-\gamma\right\}\right]
$$

The solutions smaller than $\left|z^{\min }\right|$ and larger than $1-\left|z^{\max }\right|$ have smaller perturbation sets then the basic interval $\left[z^{\min }, z^{\max }\right]$. Moreover, these smaller realization sets are nested within each other which requires specific consideration in the solution approach for determining the decision robust efficient solutions.

Having established the individual perturbation sets of the solutions $\gamma \in[0,1]$, the concept of decision robust feasibility is then defined accordingly. A feasible solution $(\gamma, \beta)$ is defined as decision robust feasible if all its realizations are salable, see (Krüger 2018, Def. 8).

As a concept of optimality for the peat-and-pots problem, decision robust efficiency is applied as defined and studied in the first publication contributing to this thesis, see (Eichfelder, Krüger, and Schöbel 2017, Def. 3).

The presented solution approach to determine the decision robust efficient solutions is based on an idea similar to the presented deterministic approach. The basic idea is to determine the decision robust efficient solutions for each $\beta \in\{0,1\}$, which is a small finite set, followed by pairwise comparisons of the obtained solutions.

To this end a finite candidate set for the decision robust efficient solutions is constructed in three steps for each $\beta \in\{0,1\}$. In the first step, the bounds of the finitely many constant intervals of the quality function are considered and from each of these points the maximum perturbation $z^{\max }$ is subtracted. The minimum robust feasible solution 0 and the maximum robust feasible solution are also added to the candidate set. In the second step, this set is reduced to those elements that are decision robust feasible. By definition of the decision robust feasible set, the resulting candidate set hence only 
contains solutions that are smaller or equal than the available quantity of compost per pot and the elements whose realizations yield a quality of $\alpha$ are kept. In the third step, the largest solutions of this set are then iteratively selected such that each solution has a higher minimum quality than the previously selected solution. The resulting finite candidate set is denoted as $\mathcal{W}(\beta)$.

This set is a valid choice for a candidate set, since, by (Krüger 2018, Prop. 12), all elements of $\mathcal{W}(\beta)$ are decision robust weakly efficient and every decision robust strictly efficient solution is an element of the candidate set. The set $\mathcal{W}(\beta)$ is then used in a straightforward way to determine the set of decisions robust [weakly $/ \cdot /$ strictly] efficient solutions $[w \mathcal{E}(\beta) / \mathcal{E}(\beta) / s \mathcal{E}(\beta)]$.

The sets of decision robust [weakly $/ \cdot /$ strictly] efficient solutions do not generally coincide. It depends on the perturbation set of each solution, whether weak and strict efficiency coincide for the solution. For all solutions $\gamma$ that are larger than the absolute value of the minimum perturbation $\left|z^{\text {min }}\right|$, the concepts of decision robust weak and strict efficiency coincide, which is shown in (Krüger 2018, Prop. 10). Solutions smaller than $\left|z^{\text {min }}\right|$ have smaller perturbation sets, see (4.10). The realization sets are nested within each other and so are their outcome sets in the objective space. Therefore, these solutions are not decision robust strictly efficient. As a consequence, there is an intervall of decision robust [weakly/·] efficient solutions if and only if there is at least one that is smaller than $\left|z^{\text {min }}\right|$. The difference between the sets of decision robust weakly and strictly efficient solutions and its connection to the solutions smaller or larger than $\left|z^{\text {min }}\right|$ is reflected in the following two theorems that state the decision robust [weakly $/ \cdot /$ strictly] efficient solutions to the peat-and-pots problem for each $\beta \in\{0,1\}$.

Theorem 4.3.2 (Krüger 2018, Th. 13). If $\mathcal{W}(\beta)$ satisfies $\mathcal{W}(\beta) \cap\left[0,-z^{\text {min }}\right]=\emptyset$, then the set

$$
\mathcal{E}(\beta)=s \mathcal{E}(\beta)=w \mathcal{E}(\beta)=\mathcal{W}(\beta)
$$

is the set of decision robust [weakly/·/strictly] efficient solutions.

Theorem 4.3.3 (Krüger 2018, Th. 14). If $\mathcal{W}(\beta)$ satisfies $\mathcal{W}(\beta) \cap\left[0,-z^{\text {min }}\right] \neq \emptyset$, the following are true.

a) The set of decision robust [weakly/·] efficient solutions is given as

$$
\mathcal{E}(\beta)=w \mathcal{E}(\beta)=\left[0, \gamma_{\mathcal{W}}^{\beta}\right] \cup \mathcal{W}(\beta),
$$

where $\gamma_{\mathcal{W}}^{\beta}:=\max \left\{\gamma \mid \gamma \in \mathcal{W}(\beta) \cap\left[0, z^{\min }\right]\right\}$.

b) The set of decision robust strictly efficient solutions is given as

$$
s \mathcal{E}(\beta)=\mathcal{W}(\beta) \backslash\left(0,-z^{\min }\right] .
$$

The results presented so far provide the solution approaches for the case study in Krüger, Castellani, Geldermann, and Schöbel 2018 with its specific requirements. In Section 5, these results are extended to further biobjective problems with one function 
that is the sum of a piecewise constant and a linear function and one linear objective function.

To this end the three assumptions that characterize the peat-and-pots problem of the case study are neglected. It is assumed that (Krüger 2018, Ass. 3), that is the GWP of the alternative input materials is lower then the GWP of the traditional materials, is a basic motivation for the considered decision problem. The solution approaches for the deterministic and the uncertain problem are then further extended to the cases that either (Krüger 2018, Ass. 1) or (Krüger 2018, Ass. 2) does not hold.

The case that the first assumption fails is of interest, because this means that the environmentally friendly input materials are more expensive than the traditional ones. The main difference between the solutions with or without this assumption is that in the former case the solution sets are finite unions of points while in the latter case, these solution sets are finite unions of intervals. This is true for the set of deterministic efficient solutions as well as the set of decision robust efficient solutions. Two algorithms are given, one to determine each set of solutions.

The absence of the second assumption means that the objective function value of adC is influenced stronger by the costs of the input materials than it is influenced by the quality of the final product. In this case, it is reasonable to reduce costs at the expense of quality. The case is hence of particular interest for applications with expensive input materials.

Two algorithms are presented to solve the problem in the absence of the second assumption. The first algorithm yields the deterministic solutions for each $\beta$. The second algorithm yields a candidate set for the decision robust efficient solutions that can be used to determine the decision robust efficient solutions by replacing the candidate set $\mathcal{W}(\beta)$ in Theorem 13 and 14 of this publication.

There are two main differences between the set of deterministic efficient solutions and the candidates set for the decision robust efficient solutions with and without (Krüger 2018, Ass. 2). The first difference is that the weakly efficient solutions and the efficient solutions do not coincide. However, the sets of efficient solutions and the set of strictly efficient solutions coincide in the deterministic and in the robust case. The second differences is that, in the algorithms, the solutions are compared with respect to their additional costs rather than with respect to their quality.

In summary, solution approaches to determine the efficient solutions for the deterministic peat-and-pots problem and three solution approaches to determine the decision robust efficient solutions for the uncertain peat-and-pots problem are presented, that depend on the presence or absence of the three assumptions defined by the case study.

The problem is first investigated under the assumptions that are required by the case study in Krüger, Castellani, Geldermann, and Schöbel 2018. The presented solution approaches can therefore be considered as the theoretical background for the latter publication. Furthermore, the problem is investigated in a more general form than required by the case study and the solution approaches are hence applicable to a wider class of problems. In particular, arbitrary piecewise constant quality functions are taken into account and it is considered that the available supply of compost is often limited 
in practice.

The problem is then investigated without the assumptions that characterize the case study and algorithms are given that yield the deterministic efficient solutions and the decision robust efficient solutions in the absence of the assumptions. They presented solution approaches are hence not only suitable for the case study but they are also of interest for further applications.

\section{Own Contribution}

The abstract, introduction and conclusion are joint work of all coauthors. The problem description and motivation in Section 2 of the publication Krüger, Castellani, Geldermann, and Schöbel 2018 was done by Francesco Castellani and Jutta Geldermann. The input data section, Section 3, was provided by Francesco Castellani. The idea of the objective functions was provided by Francesco Castellani and Jutta Geldermann and written down by the autor of this thesis. The description of the uncertainty in the decision problem and the deterministic and robust solution approaches in Sections 4 and 5 was mostly contributed by the author of this thesis with the advice of Anita Schöbel. Moreover, the determination of the deterministic and the robust efficient solutions for the case study as well as their comparison was performed and written by the author of this thesis with the advice of all coauthors.

The author of this thesis is the sole author of the publication Krüger 2018. As stated in the publication, parts of the problem motivation and description correspond to the publication Krüger, Castellani, Geldermann, and Schöbel 2018, since this publication provides the theoretical background for the former publication. The remaining results contained in this publication were developed and written down by the author of this thesis. Nevertheless the author would like to acknowledge the advice of her advisor Anita Schöbel on the results and the structure of this publication. Moreover, the cooperation with Francesco Castellani and Jutta Geldermann contributed to the problem motivation and description. 


\section{Discussion}

Minmax robustness for uncertain multiobjective optimization is a broad field of research, which is also reflected in the literature review of this thesis. Solutions to these problems depend on the type of uncertainty and on the robustness concept. In this thesis, two types of uncertainty at distinguished, namely decision and parameter uncertainty. The three considered minmax robustness concepts are decision robust efficiency, which is introduced in the first publication contributing to this thesis, point-based robust efficiency and set-based robust efficiency, and the latter two are frequently studied minmax robustness concepts for parameter uncertainty in the literature. Each of these minmax robustness concept is associated with its robust counterpart that is either an MOP or a set optimization problem.

The results of this thesis therefore contribute to minmax robustness for multiobjective optimization with decision or parameter uncertainty under consideration of robust counterparts that are either MOPs or set optimization problems. Each of the four publications of this thesis is to some extent related to each of the aforementioned types of uncertainty and robust counterparts and this is discussed subsequently. Cases in which the two types of uncertainty or robust counterparts coincide are also emphasized in this thesis, either by contributing new results or by using known results in a new context.

In the first publication Eichfelder, Krüger, and Schöbel 2017, decision robust efficiency is introduced as a robustness concept for decision uncertainty. The introduced decision robust counterpart is a set optimization problem and because it involves optimizing supremal sets, decision robust efficiency can be considered as a minmax robustness concept. The set-valued objective function of the decision robust counterpart and its supremal set are investigated with respect to convexity and semicontinuity. Also, solution approaches for three specific classes of objective functions are given. Even though this publication is mainly concerned with decision uncertainty and set-valued robust counterparts, a link to minmax robustness for parameter uncertainty is shown, which is that decision robust efficiency can be considered as a special case of set-based robust efficiency. Decision robust effiency is nevertheless worth studying in its own right, because the specific structure of the decision robust counterpart leads to results that fail for set-based robust efficiency. In particular, the result that the decision robust counterpart can be considered as a deterministic MOP in case of linear objective functions. Hence, this publication contributes to both types of uncertainty and to both types of robust counterparts with a focus on decision uncertainty and set-valued robust counterparts.

In the second publication Krüger, Schöbel, and Wiecek 2017, a multiobjective robustness gap is introduced for MOPs with decision uncertainty and robustness concepts whose robust counterpart is an MOP. For convex MOPs, the gap is shown to be zero under certain conditions including objective-wise uncertainty, and hence point-based and 
set-based robust efficiency coincide under these conditions. Lower and upper bounds for the robustness gap are given and single-objective problems with optimal objective values that correspond to these bounds are developed for the case of linear MOPs. The linear case is investigated for decision and parameter uncertainty and, since the decision robust counterpart can be considered as an MOP in case of linear objective functions according to the first publication, the developed results apply to the concept of decision robust efficiency. The results of this publication therefore mostly apply to parameter uncertainty and robust counterparts that are MOPs, but certain results are specifically developed for the case of decision robust efficiency.

The topic of the third publication Krüger, Castellani, Geldermann, and Schöbel 2018 and the fourth publication Krüger 2018 is an application of minmax robust efficiency to a biobjective problem in horticulture. The considered decision problem originates from a case study and, due to this practical background, both types of uncertainty are present. However, the parameter uncertainty in the problem is objective-wise and hence point-based and set-based robust efficiency coincide. There even exists a global worstcase scenario. Parameter and decision uncertainty can be addressed simultaneously by solving the decision robust counterpart with the worst-case scenario in place of the uncertain parameters. Analytic descriptions of the decision robust efficient solutions to the set-valued robust counterpart are given. The third publication can hence be considered as a practical study on both types of uncertainty and both types of robust counterparts. The fourth publications focuses mostly on decision uncertainty and on solving the set-valued decision robust counterpart of the application's decision problem.

So far, the connection of every publication with the topic of this thesis has been discussed, which is minmax robustness for multiobjective optimization with decision or parameter uncertainty. Moreover, there are various links between the individual publications, which are pointed out next.

The first and the second publication are connected, since the aforementioned result that the decision robust counterpart can be considered as an MOP in case of linear objective functions, is directly used in the second publication to develop a sufficient condition for the zero robustness gap and to develop the single-objective problems that can be used to calculate the lower and upper bounds.

The first publication is relevant for the third and fourth publications, because the considered decision problem in the latter publications is an application of the concept decision robust efficiency, even though the concept is slightly adapted to fit the requirements of the application. In contrast to the first publication, the perturbation sets in the third and fourth publication are not constant, but they depend on the solution. This extension of decision robust efficiency is also mentioned as further research in the conclusions of the first publication. Nevertheless, the concepts of decision robust feasibility and efficiency can be applied in this case. The main difference compared to decision robust efficiency is that different perturbation sets cause case distinctions when determining the decision robust efficient solutions. The concept of decision robust feasibility is also slightly altered in the application, where the second constraint is only required to hold for the decision variable itself rather than for all of its realizations. This is because the second constraint models a part of the application that is unaffected by decision 
uncertainty. The decision problem in the third and fourth publications can therefore be considered as an application of the first publication's minmax robustness concept. Moreover, the first publication's result on MOPs with monotonic objective functions is used in one of the solution approaches that are given in the fourth publication. This result is originally given for functions that are monotonic on the decision space and it is then extended to functions that are piecewise monotonic. Hence, there are two links between the first and the third and fourth publications: the minmax robustness concept and the solution approach for the case of monotonic objective functions.

The third and fourth publication are naturally closely related because they both investigate the same decision problem from a practical and from a theoretical perspective. 


\section{Conclusions}

This thesis contributes different aspects to the research area of minmax robust multiobjective optimization: a minmax robustness concept for MOPs with decision uncertainty is introduced and studied, it is also applied to a case study and a first definition for a multiobjective robustness gap is given and investigated.

In the first publication, the multiobjective minmax robustness concept decision robust effiency is introduced. This concept addresses decision uncertainty, whereas the majority of publications in robust multiobjective optimization address parameter uncertainty. The corresponding decision robust counterpart is a set optimization problem whose objective function can either be chosen as the set of all outcomes of a solution or as the supremal set thereof. The concept decision robust efficiency is related to existing singleand multiobjective minmax robustness concepts in the literature. For three specific problem classes, that is MOPs with linear, Lipschitz continuous or monotonic objective functions, conditions that are necessary or sufficient for decision robust efficiency are presented. These problem classes are of interest due to their properties that enable specific solution approaches and due to their relevance in applications. Linear MOPs are among the most studied problem classes in the discrete optimization literature, Lipschitz continuous objective functions are frequently considered in the continuous optimization literature and, in particular, an application of an MOP with decision and parameter uncertainty and monotonic objective functions is studied in the fourth publication of this thesis.

In the second publication, a first concept of a multiobjective robustness gap is presented. Even though there are various studies about minmax robust multiobjective optimization, no multiobjective robustness gap has been proposed until now. For convex MOPs, a lower and an upper bound for the gap are given and a sufficient condition for the zero gap is provided that supplements a major result on the single objective gap. A second and different condition for the zero robustness gap is provided for MOLPs with decision uncertainty. Also, single objective problems are formulated that yield the lower and upper bound for MOLPs. The presented multiobjective robustness gap is defined for every minmax robustness concept whose robust counterpart is an MOP, such as point-based robust efficiency, set-based robust efficiency in case of objective-wise uncertainty, decision robust efficiency if the objective function is linear and all MOPs that are affected by uncertainty only in the constraints.

In the third and fourth publications, the concept decision robust efficiency is applied to a case study. Solution approaches to determine the decision robust efficient solutions are developed and applied to calculate the solutions for the case study. The practical results show that selecting decision robust efficient solutions rather than deterministic efficient solutions is worth considering. 
The results of this thesis nevertheless point out various options for further research. In the first publication, several necessary and sufficient conditions for decision robust efficiency are presented that depend on specific properties of the objective functions. Results for linear, Lipschitz continuous, and monotonic objective functions are shown and it seems promising to investigate further classes of objective functions such as convex ones. Moreover, decision uncertainty is modeled in the first publication by assuming that each solution is affected by exactly the same perturbations set. Conversely, the application in the third and fourth publications shows that perturbation sets that depend on the solution are also worth studying. The concept of decision robust feasibility and decision robust efficiency can equivalently be used for these individual perturbation sets and solving the resulting robust counterpart is a topic that offers plenty of novel opportunities and challenges.

The proposed multiobjective robustness gap is suitable for various multiobjective robustness concepts and it can be approximated by computing its lower and upper bounds. However, the multiobjective robustness gap is only applicable to robust counterparts that are MOPs and minmax robustness concepts with set-valued robust counterparts require a different definition of a multiobjective robustness gap. Moreover, the proposed robustness gap measures the distance between Pareto sets and to define a robust Pareto set for set-valued robust counterparts is also a topic of future research.

As mentioned in the third publication of this thesis, uncertain decision problems with environmental and economic objective functions have more applications than the investigated case study in the plant nursery, for example in the area of food packaging. The presented application of decision robust efficiency thus shows that applications of this and other minmax robustness concepts are of interest from a theoretical and a practical perspective. 


\section{Bibliography}

Asprion, N., S. Blagov, R. Böttcher, J. Schwientek, J. Burger, E. von Harbou, and M. Bortz (2017). "Simulation and Multi-criteria Optimization under Uncertain Model Parameters of a Cumene Process". In: Chemie Ingenieur Technik 89.5, pp. 665-674.

Aubin, J. and H. Frankowska (1990). Set-valued analysis. Boston, Basel, Berlin: Birkhäuser.

Avigad, G. and J. Branke (2008). "Embedded Evolutionary Multi-objective Optimization for Worst Case Robustness". In: Proceedings of the 10th Annual Conference on Genetic and Evolutionary Computation. GECCO '08. New York, NY, USA: ACM, pp. 617-624.

Barrico, C. and C. Antunes (2006). "Robustness Analysis in Multi-Objective Optimization Using a Degree of Robustness Concept". In: IEEE Congress on Evolutionary Computation. CEC 2006. IEEE Computer Society, pp. $1887-1892$.

Bauschke, H. H., D. R. Luke, H. M. Phan, and X. Wang (2013). "Restricted Normal Cones and the Method of Alternating Projections: Theory". In: Set-Valued and Variational Analysis 21.3, pp. 431-473.

Bayer, H. G. and B. Sendhoff (2007). "Robust Optimization - A Comprehensive Survey". In: Computer Methods in Applied Mechanics and Engineering 196.33-34, pp. 31903218.

Ben-Tal, A., L. E. Ghaoui, and A. Nemirovski (2009). Robust Optimization. Princeton and Oxford: Princeton University Press.

Ben-Tal, A. and D. den Hertog (2011). "Immunizing conic quadratic optimization problems against implementation errors". In: CentER Working Paper Series 2011-060.

Ben-Tal, A. and A. Nemirovski (1998). "Robust convex optimization". In: Mathematics of Operations Research 23.4, pp. 769-805.

- (1999). "Robust solutions of uncertain linear programs". In: Operations Research Letters 25, pp. 1-13.

- (2000). "Robust Solutions of linear Programming Problems contaminated with uncertain data". In: Mathematical Programming A 88, pp. 411-424.

- (2002). "Robust optimization - methodology and applications". In: Mathematical Programming 92.3, pp. 453-480.

Benoist, J. and N. Popovici (2003). "Characterizations of convex and quasiconvex setvalued maps". In: Math. Meth. of OR 57.3, pp. 427-435.

Bertsimas, D., D. B. Brown, and C. Caramanis (2011). "Theory and Applications of Robust Optimization". In: SIAM Review 53.3, pp. 464-501.

Bertsimas, D. and O. Nohadani (2010). "Robust optimization with simulated annealing". In: Journal of Global Optimization 48.2, pp. 323-334.

Bertsimas, D., O. Nohadani, and K. M. Teo (2007). "Robust optimization in electromagnetic scattering problems". In: Journal of Applied Physics 101.7, p. 074507. 
Bertsimas, D., O. Nohadani, and K. M. Teo (2010a). "Nonconvex Robust Optimization for Problems with Constraints". In: INFORMS Journal on Computing 22.1, pp. 4458.

- (2010b). "Robust Optimization for Unconstrained Simulation-Based Problems". In: Operations Research 58.1, pp. 161-178.

Bertsimas, D. and M. Sim (2004). "The price of robustness". In: Operations Research 52.1 , pp. $35-53$.

Besharati, B. and S. Azarm (2006). "Worst case deterministic feasibility and multiobjective robustness measures for engineering design optimisation". In: International Journal of Reliability and Safety 1.1-2, pp. 40-58.

Bischoff, M., J. Jahn, and E. Köbis (2017). "Hard uncertainties in multiobjective layout optimization of photovoltaic power plants". In: Optimization 66.3, pp. 361-380.

Bitran, G. R. (1980). "Linear Multiple Objective Problems with Interval Coefficients". In: Management Science 26, pp. 694-706.

Bokrantz, R. and A. Fredriksson (2017). "Necessary and sufficient conditions for Pareto efficiency in robust multiobjective optimization". In: European Journal of Operational Research 262.2, pp. $682-692$.

Botte, M. and A. Schöbel (2016). Dominance for Multi-Objective Robust Optimization Concepts. Tech. rep. Preprint-Reihe, Institut für Numerische und Angewandte Mathematik, Georg-August Universität Göttingen.

Caballero, R., E. Cerdá, M. M. Muñoz, L. Rey, and I. M. Stancu-Minasian (2001). "Efficient Solution Concepts and Their Relations in Stochastic Multiobjective Programming". In: Journal of Optimization Theory and Applications 110.1, pp. 53-74.

Chen, W., J. Unkelbach, A. Trofimov, T. Madden, H. Kooy, T. Bortfeld, and D. Craft (2012). "Including robustness in multi-criteria optimization for intensity-modulated proton therapy". In: Physics in medicine and biology 57.3, p. 591.

Chuong, T. D. (2016). "Optimality and duality for robust multiobjective optimization problems". In: Nonlinear Analysis: Theory, Methods \& Applications 134, pp. $127-$ 143.

Crespo, O., J. Bergez, and F. Garcia (2010). "Multiobjective optimization subject to uncertainty: Application to irrigation strategy management". In: Computers and Electronics in Agriculture 74.1, pp. 145 -154.

Das, I. (1997). "Nonlinear multicriteria optimization and robust optimality". PhD thesis. Rice University.

Deb, K. and H. Gupta (2005). "Searching for Robust Pareto-Optimal Solutions in Multiobjective Optimization." In: EMO. Vol. 2005, pp. 150-164.

- (2006). "Introducing Robustness in Multi-Objective Optimization". In: Evolutionary Computation 14.4, pp. 463-494.

Doolittle, E. K., G. M. Dranichak, K. Muir, and M. M. Wiecek (2016). "A note on robustness of the min-max solution to multi-objective linear programs". In: International Journal of Multicriteria Decision Making 6.4, pp. 343-365.

Doolittle, E. K., H. L. M. Kerivin, and M. M. Wiecek (2015). A robust multiobjective optimization problem with application to Internet routing. Tech. rep. TR2015-11ed.hk.mw. Clemson University. 
Durea, M. (2007). "On the existence and stability of approximate solutions of perturbed vector equilibrium problems". In: Journal of Mathematical Analysis and Applications $333.2,1165-1179$.

Edgeworth, F. Y. (1881). Mathematical psychics: An essay on the application of mathematics to the moral sciences. Vol. 10. Kegan Paul.

Ehrgott, M. (2005). Multicriteria Optimization. Springer, Berlin, Heidelberg.

Ehrgott, M., J. Ide, and A. Schöbel (2014). "Minmax Robustness for Multi-objective Optimization Problems". In: European Journal of Operational Research 239, pp. 17 31.

Eichfelder, G. and J. Jahn (2012). "Vector Optimization Problems and Their Solution Concepts". In: Recent Developments in Vector Optimization. Ed. by Q. H. Ansari and J. C. Yao. Berlin Heidelberg: Springer, pp. 1-27.

Eichfelder, G., C. Krüger, and A. Schöbel (2017). "Decision uncertainty in multiobjective optimization". In: Journal of Global Optimization 69.2, pp. 485-510.

Fadel, G., I. Haque, V. Blouin, and M. M. Wiecek (2005). "Multi-criteria multi-scenario approaches in the design of vehicles". In: Proceedings of 6th World Congresses of Structural and Multidisciplinary Optimization, Rio de Janeiro, Brazil. Available online at http://www. wcsmo6. org/papers/6391. pdf.

Fakhar, M., M. Mahyarinia, and J. Zafarani (2017). "On Nonsmooth Robust Multiobjective Optimization Under Generalized Convexity With Applications To Portfolio Optimization". In: European Journal of Operational Research, pp. -.

Farshidi, A., L. Rakai, L. Behjat, and D. Westwick (2016). "Variation-aware clock network buffer sizing using robust multi-objective optimization". In: Optimization and Engineering 17.2, pp. 473-500.

Fischetti, M. and M. Monaci (2009). "Light Robustness". In: Robust and Online Largescale Optimization. Ed. by R. K. Ahuja, R. H. Möhring, and C. D. Zaroliagis. Vol. 5868. Lecture Notes in Computer Science. Springer, Berlin, Heidelberg, pp. 6184.

Fliege, J. and R. Werner (2014). "Robust multiobjective optimization \& applications in portfolio optimization". In: European Journal of Operational Research 234.2, pp. 422 $-433$.

Gabrel, V., C. Murat, and A. Thiele (2014). "Recent advances in robust optimization: An overview". In: European Journal of Operational Research 235.3, pp. 471 -483.

Gaspar-Cunha, A. and J. A. Covas (2008). "Robustness in multi-objective optimization using evolutionary algorithms". In: Computational Optimization and Applications 39.1, pp. $75-96$.

Geoffrion, A. M. (1968). "Proper efficiency and the theory of vector maximization". In: Journal of Mathematical Analysis and Applications 22.3, pp. 618 -630.

Georgiev, P., D. Luc, and P. Pardalos (2013). "Robust aspects of solutions in deterministic multiple objective linear programming". In: European Journal of Operational Research 229.1, pp. $29-36$.

Goberna, M. A., V. Jeyakumar, G. Li, and J. Vicente-Pérez (2014). "Robust Solutions of MultiObjective Linear Semi-Infinite Programs under Constraint Data Uncertainty". In: SIAM Journal on Optimization 24.3, pp. 1402-1419. 
Goberna, M. A., V. Jeyakumar, G. Li, and J. Vicente-Pérez (2015). "Robust solutions to multi-objective linear programs with uncertain data". In: European Journal of Operational Research 242.3.

Goerigk, M. and A. Schöbel (2016). "Algorithm Engineering in Robust Optimization". In: Algorithm Engineering: Selected Results and Surveys. Ed. by L. Kliemann and P. Sanders. Vol. 9220. LNCS State of the Art, pp. 245-279. URL: http://arxiv . org/abs/1505.04901.

Gunawan, S. and S. Azarm (2005). "Multi-objective robust optimization using a sensitivity region concept". In: Structural and Multidisciplinary Optimization 29.1, pp. 5060.

Ha, T. and J. Jahn (2011). "New order relations in set optimization". In: J. Optim. Theory Appl. 148, pp. 209-236.

Hassanzadeh, F., H. Nemati, and M. Sun (2013). "Robust Optimization for Multiobjective Programming Problems with Imprecise Information". In: Procedia Computer Science 17, pp. $357-364$.

- (2014). "Robust optimization for interactive multiobjective programming with imprecise information applied to R\&D project portfolio selection". In: European Journal of Operational Research 238.1, pp. $41-53$.

Hladík, M. (2010). "On necessarily efficient solutions in interval multiobjective linear programming". In: Proceedings of the 25th Mini-EURO conference on uncertainty and robustness in planning and decision making, URPDM, pp. 1-10.

- (2012). "Complexity of necessary efficiency in interval linear programming and multiobjective linear programming". In: Optimization Letters 6.5, pp. 893-899.

Hombach, L. E., C. Büsing, and G. Walther (2017). "Robust and sustainable supply chains under market uncertainties and different risk attitudes - A case study of the German biodiesel market". In: European Journal of Operational Research.

$\mathrm{Hu}$, J. and S. Mehrotra (2012). "Robust and Stochastically Weighted Multiobjective Optimization Models and Reformulations". In: Operations Research 60.4, pp. 936953.

Huang, S.-Y. and J. Teghem (2012). Stochastic versus fuzzy approaches to multiobjective mathematical programming under uncertainty.

Ida, M. (1996). "Generation of efficient solutions for multiobjective linear programming with interval coefficients". In: Proceedings of the 35th SICE Annual Conference. International Session Papers, pp. 1041-1044.

Ida, M (2003). "Portfolio selection problem with interval coefficients". In: Applied Mathematics Letters 16.5, pp. $709-713$.

Ide, J. (2014). "Concepts of Robustness for Uncertain Multi-Objective Optimization". $\mathrm{PhD}$ thesis. Universität Göttingen.

Ide, J. and E. Köbis (2014). "Concepts of Efficiency for Uncertain Multi-Objective Optimization Problems based on Set Order Relations". In: Mathematical Methods of Operations Research, pp. 1-29. ISSN: 1432-2994. DOI: 10.1007/s00186-014-0471-z.

Ide, J., E. Köbis, D. Kuroiwa, A. Schöbel, and C. Tammer (2014). "The relationship between multi-objective robustness concepts and set valued optimization". In: Fixed 
Point Theory and Applications 2014.83. DOI: 10.1186/1687-1812-2014-83. URL: http://www.fixedpointtheoryandapplications.com/content/2014/1/83.

Ide, J. and A. Schöbel (2016). "Robustness for uncertain multi-objective optimization: A survey and analysis of different concepts". In: OR Spectrum 38.1, pp. 235-271.

Ide, J., M. Tiedemann, S. Westphal, and F. Haiduk (2015). "An application of deterministic and robust optimization in the wood cutting industry". In: $4 O R$ 13.1, pp. $35-$ 57.

Jahn, J. (2011). Vector Optimization (2. ed.). Berlin, Heidelberg: Springer.

Kabgani, A. and M. Soleimani-damaneh (2018). "Characterization of (weakly/properly/robust) efficient solutions in nonsmooth semi-infinite multiobjective optimization using convexificators". In: Optimization 67.2, pp. 217-235.

Kalantari, M., C. Dong, and I. Davies (2016). "Multi-objective robust optimisation of unidirectional carbon/glass fibre reinforced hybrid composites under flexural loading". In: Composite Structures 138, pp. 264-275.

Khan, A., C. Tammer, and C. Zălinescu (2015). Set-valued Optimization - an Introduction with Applications. Berlin Heidelberg: Springer.

Kim, M. H. (2013a). "Duality theorem and vector saddle point theorem for robust multiobjective optimization problems". In: Communications of the Korean Mathematical Society 28.3, pp. 597-602.

- (2013b). "Robust duality for generalized invex programming problems". In: Communications of the Korean Mathematical Society 28.2, pp. 419-423.

Klamroth, K., E. Köbis, A. Schöbel, and C. Tammer (2017). "A unified approach to uncertain optimization". In: European Journal of Operational Research 260.2, pp. 403420.

Kouvelis, P. and G. Yu (1997). Robust Discrete Optimization and Its Applications. Nonconvex Optimization and its Applications. Kluwer Academic Publishers, Dordrecht.

Krüger, C. (2018). Peat and pots: Analysis of robust solutions for a biobjective problem in agriculture. Tech. rep. 2018-5. Preprint-Reihe, Institut für Numerische und Angewandte Mathematik, Georg-August Universität Göttingen.

Krüger, C., F. Castellani, J. Geldermann, and A. Schöbel (2018). Peat and Pots: An application of robust multiobjective optimization to a mixing problem in agriculture. Tech. rep. 2018-4. Preprint-Reihe, Institut für Numerische und Angewandte Mathematik, Georg-August Universität Göttingen.

Krüger, C., A. Schöbel, and M. M. Wiecek (2017). The robustness gap for uncertain multiobjective optimization. Tech. rep. 2017-3. Preprint-Reihe, Institut für Numerische und Angewandte Mathematik, Georg-August Universität Göttingen.

Kuhn, K., A. Raith, M. Schmidt, and A. Schöbel (2016). "Bicriteria robust optimization". In: European Journal of Operational Research 252, pp. 418-431. URL: http: //dx.doi.org/10.1016/j.ejor.2016.01.015.

Kuroiwa, D. (1996). "Convexity for set-valued maps." In: Appl. Math. Lett. 9.2, pp. 97 101.

- (1998). "The natural criteria in set-valued optimization." In: RIMS Kokyuroku 1031, pp. 85-90. 
Kuroiwa, D. and G. M. Lee (2012). "On Robust Multiobjective Optimization". In: Vietnam Journal of Mathematics 40.2\&3, pp. 305-317.

Kutateladze, S. (1979). "Convex ع-programming". In: Soviet Math Dokl 20.2, 391-393.

Lee, J. H. and G. M. Lee (2016). "On optimality conditions and duality theorems for robust semi-infinite multiobjective optimization problems". In: Annals of Operations Research, pp. 1-20.

Lewis, A. S. (2002). Robust Regularization. Tech. rep. Available online at http:// people.orie.cornell . edu/aslewis/publications/2002.html. Cornell University, Ithaca, NY.

Lewis, A. S. and C. H. J. Pang (2009). "Lipschitz Behavior of the Robust Regularization". In: SIAM Journal on Control and Optimization 48.5, pp. 3080-3105.

Li, M. and S. Azarm (2008). "Multiobjective collaborative robust optimization with interval uncertainty and interdisciplinary uncertainty propagation". In: Journal of mechanical design 130.8, p. 081402.

Löhne, A. (2011). Vector Optimization with Infimum and Supremum. Vector Optimization. Springer, Heidelberg, Berlin.

Luc, D. (1989). Theory of vector optimization. Vol. 319. Lecture Notes in Economics and Mathematical Systems. Springer-Verlag, Berlin.

Majewski, D. E., M. Wirtz, M. Lampe, and A. Bardow (2017). "Robust multi-objective optimization for sustainable design of distributed energy supply systems". In: Computers $\mathscr{G}$ Chemical Engineering 102, pp. 26 -39.

Mavrotas, G., O. Pechak, E. Siskos, H. C. Doukas, and J. E. Psarras (2015). "Robustness analysis in Multi-Objective Mathematical Programming using Monte Carlo simulation". In: European Journal of Operational Research 240.1, pp. 193-201.

Miettinen, K. (2012). Nonlinear multiobjective optimization. Vol. 12. Springer.

Nieuwenhuis, J. (1980). "Supremal points and generalized duality". In: Optimization 11.1, pp. 41-59.

Nikulin, Y., O. Karelkina, and M. Mäkelä (2013). "On accuracy, robustness and tolerances in vector Boolean optimization". In: European Journal of Operational Research (2013) 224, pp. 449-457.

Oliveira, C. and C. H. Antunes (2007). "Multiple objective linear programming models with interval coefficients - an illustrated overview". In: European Journal of Operational Research 181.3, pp. $1434-1463$.

Palma, C. D. and J. D. Nelson (2010). "Bi-objective multi-period planning with uncertain weights: a robust optimization approach". In: European Journal of Forest Research 129.6, pp. 1081-1091.

Pareto, V. (1906). Manuale di economia politica. Vol. 13. English translation: V. Pareto. Manual of Political Economy, translated by A. S. Schwier. Augustus M. Kelley Publishers, New York, 1971. Societa Editrice.

Pourkarimi, L. and M. Soleimani-damaneh (2016). "Robustness in deterministic multiobjective linear programming with respect to the relative interior and angle deviation". In: Optimization 65.11, pp. 1983-2005.

Raith, A., M. Schmidt, A. Schöbel, and L. Thom (2017). Extensions of Labeling Algorithms for Multi-objective Uncertain Shortest Path Problems. Tech. rep. 2017- 
2. Preprint-Reihe, Institut für Numerische und Angewandte Mathematik, GeorgAugust Universität Göttingen.

- (2018). "Multi-objective minmax robust combinatorial optimization with cardinalityconstrained uncertainty". In: European Journal of Operational Research 267, pp. 628642. URL: https://doi.org/10.1016/j.ejor.2017.12.018.

Rivaz, S. and M. A. Yaghoobi (2013). "Minimax regret solution to multiobjective linear programming problems with interval objective functions coefficients". In: Central European Journal of Operations Research 21.3, pp. 625-649.

Rockafellar, R. T. (2015). Convex analysis. Princeton Landmarks in Mathematics and Physics. Princeton university press.

Rodríguez-Marín, L. and M. Sama (2007). " $(\Lambda, C)$-contingent derivatives of set-valued maps". In: J. Math. Anal. Appl. 335, pp. 974-989.

Sawaragi, Y., H. Nakayama, and T. Tanino (1985). Theory of multiobjective optimization. Vol. 176. Elsevier.

Schmidt, M., A. Schöbel, and L. Thom (2018). Min-ordering and max-ordering scalarization methods for multi-objective robust optimization. Tech. rep. 2018-3. PreprintReihe, Institut für Numerische und Angewandte Mathematik, Georg-August Universität Göttingen.

Schöbel, A. (2014). "Generalized light robustness and the trade-off between robustness and nominal quality". In: MMOR 80.2, pp. 161-191.

Soyster, A. L. (1973). "Convex programming with set-inclusive constraints and applications to inexact linear programming". In: Operations Research 21, pp. 1154-1157.

Stinstra, E. and D. den Hertog (2008). "Robust optimization using computer experiments". In: European Journal of Operational Research 191.3, pp. 816 -837.

Thom, L. (2018). "Solution Methods for Multi-Objective Robust Combinatorial Optimization". PhD thesis. Universität Göttingen.

Wang, F., S. Liu, and Y. Chai (2015). "Robust counterparts and robust efficient solutions in vector optimization under uncertainty". In: Operations Research Letters 43, pp. 293-298.

Wang, L., Q. Li, R. Ding, M. Sun, and G. Wang (2017). "Integrated scheduling of energy supply and demand in microgrids under uncertainty: A robust multi-objective optimization approach". In: Energy 130, pp. 1 -14.

Wei, H.-Z., C.-R. Chen, and S.-J. Li (2017). "A Unified Characterization of Multiobjective Robustness via Separation". In: Journal of Optimization Theory and Applications.

Wiecek, M. M. and G. M. Dranichak (2016). "Robust Multiobjective Optimization for Decision Making Under Uncertainty and Conflict". In: Optimization Challenges in Complex, Networked and Risky Systems. INFORMS. Chap. 4, pp. 84-114.

Witting, K. (2012). "Numerical algorithms for the treatment of parametric multiobjective optimization problems and applications". PhD thesis. Paderborn: Universität Paderborn.

Witting, K., S. Ober-Blöbaum, and M. Dellnitz (2013). "A variational approach to define robustness for parametric multiobjective optimization problems". In: Journal of Global Optimization 57.2, pp. 331-345. 
Xidonas, P., G. Mavrotas, C. Hassapis, and C. Zopounidis (2017). "Robust multiobjective portfolio optimization: A minimax regret approach". In: European Journal of Operational Research 262.1, pp. 299 -305.

$\mathrm{Yu}, \mathrm{H}$. and H. Liu (2013). "Robust Multiple Objective Game Theory". English. In: Journal of Optimization Theory and Applications 159.1, pp. 272-280. ISSN: 00223239 .

Zamani, M., M. Soleimani-damaneh, and A. Kabgani (2015). "Robustness in nonsmooth nonlinear multi-objective programming". In: European Journal of Operational Research 247.2 , pp. $370-378$.

Zimmermann, H.-J. (2012). Fuzzy sets, decision making, and expert systems. Vol. 10. Springer Science \& Business Media. 
Addenda 


\title{
A. Decision Uncertainty in Multiobjective Optimization
}

\author{
G. Eichfelder, C. Krüger, A. Schöbel
}




\title{
Decision Uncertainty in Multiobjective Optimization
}

\author{
Gabriele Eichfelder*1, Corinna Krüger ${ }^{\dagger 2}$, and Anita Schöbel ${ }^{\S 2}$ \\ ${ }^{1}$ Technische Universität Ilmenau, Ilmenau, Germany \\ ${ }^{2}$ University of Goettingen, Göttingen, Germany
}

\begin{abstract}
In many real-world optimization problems, a solution cannot be realized in practice exactly as computed, e.g., it may be impossible to produce a board of exactly $3.546 \mathrm{~mm}$ width. Whenever computed solutions are not realized exactly but in a perturbed way, we speak of decision uncertainty. We study decision uncertainty in multiobjective optimization problems and we propose the concept of decision robust efficiency for evaluating the robustness of a solution in this case. This solution concept is defined by using the framework of set-valued maps. We prove that convexity and continuity are preserved by the resulting set-valued maps. Moreover, we obtain specific results for particular classes of objective functions that are relevant for solving the set-valued problem. We furthermore prove that decision robust efficient solutions can be found by solving a deterministic problem in case of linear objective functions. We also investigate the relationship of the proposed concept to other concepts in the literature.
\end{abstract}

\section{Introduction}

When applying mathematical optimization methods to real world problems, several difficulties have to be considered. We study a class of problems where two specific difficulties occur simultaneously. First, the problem can have several conflicting objectives, leading to a multiobjective optimization problem. Second, a calculated solution may only be realized within some accuracy instead of being put into practice exactly. Realizations of calculated solutions have to be considered uncertain in this case. In the following, we refer to this kind of uncertainty as decision uncertainty since the decision variables are the source of uncertainty inside the problem. We distinguish decision uncertainty from parameter uncertainty in optimization problems, where the values of parameters are not known at the time

\footnotetext{
*Email address: Gabriele.Eichfelder@tu-ilmenau.de

†Corresponding author; Email address: ckrueger@math.uni-goettingen.de

‡Supported by DFG RTG 1703 "Resource Efficiency in Corporate Networks"

$\S$ Email address: schoebel@math.uni-goettingen.de
} 
a problem is solved, see, e.g., [BTGN09] for parameter uncertainty in single-objective optimization and [EIS14], [FW14] or [GJLVP14] for parameter uncertainty in multiobjective optimization.

In recent years, multiobjective optimization problems including parameter uncertainty have been studied in various ways. In [KL12] and [GJLVP14], the authors consider for each solution the vector consisting in each component of the worst case of the associated objective in place of the uncertain objective. The authors of [EIS14] and [AB08] compare solutions by the sets of their possible outcome vectors. Solutions that are effficient for all realizations of uncertainty are studied, e.g., in [GLP13]. For a survey of different concepts of robustness in the literature, we refer the reader to [IS16] and [WD16].

Single-objective optimization problems including decision uncertainty have been treated with minmax robustness under different names. Common names are "robust optimization with implementation error", see, e.g., [BTH11, BTN02, BNT07, BNT10a, BNT10b, SdH08], or robust regularization, see [BS07, LP09]. Also [Das97, BN10] deal with decision uncertainty. Decision uncertainty in multiobjective optimization has been addressed by evaluating the mean or integral of each objective over the set of possible values of a solution, see, e.g. [DG06], or by sensitivity analysis, see, e.g., [BA06]. However, until now, there exists no worst-case robustness approach for decision uncertainty in multiobjective optimization.

In this work, we investigate decision uncertainty in multiobjective optimization with a minmax robustness approach based on set-valued optimization, i.e., we define and analyze a robust counterpart for this kind of problem and we present approaches to solve it. The conceptual idea of the presented robustness concept, which we call decision robust efficiency, is based on a minmax robustness approach to parameter uncertainty in multiobjective optimization introduced by [EIS14]. However, exploiting the specific structure arising in problems with decision uncertainty, we obtain results that are generally not valid for problems with parameter uncertainty.

In the following paragraphs we sketch practical applications of multiobjective problems that are affected by decision uncertainty. Definitions from set-valued optimization are recalled in Section 2. Following on that, the robustness concept of decision robust efficiency is developed. In Section 3, the set-valued functions that correspond to decision robust efficiency are investigated with respect to continuity and convexity properties. Section 4 relates the presented robustness concept to approaches in the literature. Solution approaches for problem classes with specific objective functions are studied in Section 5.

\section{Applications}

We present two real-world applications for multiobjective decision uncertainty. First, we present an application in the Lorentz force velocimetry framework and second, we present the Growing Media Mixing Problem for plant nurseries. Both applications can be modeled as biobjective optimization problems with decision uncertainty. 
Lorentz Force Velocimetry (LFV) Framework: The LFV is an electromagnetic non-contact flow measurement technique for electrically conducting fluids. This is especially suited for corrosive or extremely hot fluids like glass melts or acidic mixtures which can damage other measurement setups [DTE14]. The magnetic field of the permanent magnets interacts with an electrically conducting fluid which moves through a channel, see Figure 1. Eddy currents develop and the resulting secondary magnetic field acts on the

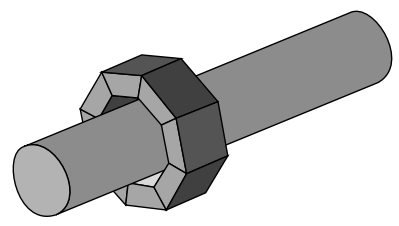

Figure 1: Illustration of a non-contact flow measurement system with 8 magnets.

magnet system. The Lorentz force breaks the fluid and an equal but opposite force deflects the magnet system, which can be measured. Fluids with a small electrical conductivity produce only very small Lorentz forces. Thus, a sensitive balance system is required for a reliable measurement. This limits the weight of the external magnet system. Hence, we obtain two conflicting goals: maximize the Lorentz force and minimize the magnetization. As variables, the direction of the magnetization of each magnet and the magnetization have to be optimized.

Figure 1 gives a simple arrangement of eight permanent magnets around a channel. In practice an (optimally) chosen magnetic direction cannot be realized in the desired accuracy, as magnets can only be produced with a guarantee of the magnetic directions within some tolerance interval. Therefore, decision uncertainty has to be taken into account here, in order to avoid solutions with practical realizations that are far from being efficient in the worst case.

Growing Media Mixing Problem for a Plant Nursery: For each plant species, a plant nursery that produces potted plants has to decide the type of planter pot and the type of growing media to be used. First, possible types of planter pots are plastic pots made from fossil ressources and bio-degradable planter pots. The former are cheaper, while the latter are more eco-friendly. Second, the growing media can be chosen as a mixture of peat and compost, where the feasible mixing ratio lies between $100 \%$ peat and $33 \%$ peat plus $66 \%$ compost. The associated decision problem consists of determining one type of planter pot and one mixing ratio for each plant species such that two objectives, i.e., costs and global warming potential, are minimized.

However, a chosen mixing ratio can usually not be put into practice exactly. Workers in plant nurseries are used to work rather fast than exactly and the different types of soil are often mixed in a rough fashion only. Hence, the mixing ratio will vary in each planter pot. Because the mixing ratio influences the quality of the raised plant and therefore its selling price, decision uncertainty has to be taken into account in this biobjective optimization problem. A detailed study of this problem can be found in [CKGS17]. 


\section{Decision Robust Efficiency}

We consider the (deterministic) multiobjective optimization problem

$$
(\mathcal{P}) \quad \min _{x \in \Omega} f(x)
$$

with feasible set $\Omega \subseteq \mathbb{R}^{n}$ and objective function $f: \Omega \rightarrow \mathbb{R}^{k}$. We want to take into account the fact that the decision variables in $(\mathcal{P})$ are uncertain, i.e., they can not be realized exactly as computed.

The practical realization of a solution $x \in \mathbb{R}^{n}$ is composed of $x$ and a perturbation from a fixed perturbation set $Z \subseteq \mathbb{R}^{n}$, i.e., the realization of a solution $x$ is an element of the set $\{x\}+Z=\{x+z \mid z \in Z\}$. Because a solution might as well be put into practice exactly as planned, we require $0 \in Z$. Decision uncertainty in $(\mathcal{P})$ is modeled by considering the family of optimization problems

$$
\left(\mathcal{P}(z) \min _{x+z \in \Omega} f(x+z), \quad z \in Z\right) .
$$

The goal is to find a solution $x$ without knowing the value of the perturbation $z$. We want to hedge against the worst case. Addressing decision uncertainty within the framework of robust optimization, we define:

Definition 1. A point $x \in \mathbb{R}^{n}$ is called decision robust feasible for $(\mathcal{P}(z), z \in Z)$ if $\{x+z \mid z \in Z\} \subseteq \Omega$, i.e., if all realizations of $x$ are feasible solutions. We call

$$
X:=\left\{x \in \mathbb{R}^{n} \mid x+z \in \Omega \text { for all } z \in Z\right\}
$$

the set of decision robust feasible solutions.

Definition 1 takes a conservative point of view since $x$ is required to be feasible for every perturbation that may occur. This conceptual idea corresponds to strict robust feasibility by [BTGN09] for single-objective robust optimization with parameter uncertainty and is studied for multiobjective optimization with parameter uncertainty in [GJLVP14].

In the remainder of the paper, we make the following two assumptions:

- We assume $Z \subseteq \mathbb{R}^{n}$ is a compact set and $0 \in Z$. Hence, $X \subseteq \Omega$.

- We furthermore assume $X \neq \emptyset$.

For each decision robust feasible solution $x \in X$ we define the image set of all realizations of $x$ as

$$
f_{Z}(x):=\{f(x+z) \mid z \in Z\} .
$$

In order to develop a solution concept for $(\mathcal{P}(z), z \in Z)$, we recall solution concepts from both, multiobjective optimization and set-valued optimization. 


\subsection{Approach using Ideas from Parameter Uncertainty}

In the following, we write $\operatorname{cl}(\cdot)$, int $(\cdot)$ and $\operatorname{bd}(\cdot)$ for the closure, the interior and the boundary of a set, respectively. Furthermore, we denote the open and closed ball of radius $\varepsilon>0$ for a given norm $\|\cdot\|$ on $\mathbb{R}^{k}$ around a point $\bar{y} \in \mathbb{R}^{k}$ as $B(\bar{y}, \varepsilon):=\left\{y \in \mathbb{R}^{k} \mid\|y-\bar{y}\|<\varepsilon\right\}$ and $\bar{B}(\bar{y}, \varepsilon):=\left\{y \in \mathbb{R}^{k} \mid\|y-\bar{y}\| \leq \varepsilon\right\}$.

Recall that a nonempty set $K$ is called a cone if $\lambda k \in K$ for all $\lambda \geq 0$ and all $k \in K$, pointed if $K \cap(-K)=\{0\}$, and solid if int $(K) \neq \emptyset$. A cone $K$ is convex if and only if $K+K=K$. It is well known that for a convex pointed cone $K$ we have $K+\operatorname{int}(K)=\operatorname{int}(K)$ as well as $K \backslash\{0\}+K=K \backslash\{0\}$ and that the sets int $(K)$ and $K \backslash\{0\}$ are also convex (see for instance [Jah11]). A closed convex pointed and solid cone $K \subseteq \mathbb{R}^{k}$ defines a partial order on $\mathbb{R}^{k}$. We consider the following relations:

$$
x[</ \leq / \leqq] y \quad \Leftrightarrow \quad y-x \in[\operatorname{int}(K) / K \backslash\{0\} / K]
$$

A possible choice for $K \subseteq \mathbb{R}^{k}$ is $K=\mathbb{R}_{+}^{k}:=\left\{y \in \mathbb{R}^{k} \mid y_{i} \geq 0, \forall 1 \leq i \leq k\right\}$. Then the partial order introduced by $K$ is also called the component-wise or natural order on $\mathbb{R}^{k}$.

Given a closed, convex, pointed and solid cone $K \subseteq \mathbb{R}^{k}$, the classical optimality concepts for the deterministic multiobjective problem $(\mathcal{P})$ are given in the following definition. We remark that efficiency is often referred to as (Edgeworth-) Pareto minimality.

Definition 2. A solution $x^{*} \in \Omega$ is called [weakly/./strictly] efficient for $(\mathcal{P})$ if there is no $x \in \Omega \backslash\left\{x^{*}\right\}$ with the property $f(x) \in\left\{f\left(x^{*}\right)\right\}-[\operatorname{int}(K) / K \backslash\{0\} / K]$.

For robust multiobjective optimization with parameter uncertainty, [EIS14] propose a solution concept that generalizes Definition 2 in the following way: the outcome vector $f(x)$ in Definition 2 is replaced by the set of all possible outcomes for all scenarios. Correspondingly, for robust multiobjective optimization with decision uncertainty, we replace the outcome vector $f(x)$ in Definition 2 by the outcome set $f_{Z}(x)$ to obtain the solution concept decision robust efficiency in the following definition.

Definition 3. A solution $x^{*} \in X$ is called a decision robust [weakly/./strictly] efficient solution of $(\mathcal{P}(z), z \in Z)$, if there is no $x \in X \backslash\left\{x^{*}\right\}$ with the property

$$
f_{Z}(x) \subseteq f_{Z}\left(x^{*}\right)-[\operatorname{int}(K) / K \backslash\{0\} / K] .
$$

In Section 2.2 we motivate that Definition 3 indeed makes sense for handling decision uncertainty. We do this by showing that the set of decision robust efficient solutions of $(\mathcal{P}(z), z \in Z)$ equals the set of optimal solutions of a deterministic set-valued optimization problem.

\subsection{Approach using Set-Valued Optimization}

Considering the image set of all realizations $f_{Z}(x)$ for all $x \in X$ defines a set-valued map $f_{Z}: X \rightrightarrows \mathbb{R}^{k}$. We define the Robust Counterpart (RC) of the decision uncertain multiobjective problem $(\mathcal{P}(z), z \in Z)$ as the set-valued optimization problem

(RC) $\quad \min _{x \in X} f_{Z}(x)$. 
Next, we see that the decision robust efficient solutions of $(\mathcal{P}(z), z \in Z)$ are exactly the optimal solutions to the set-valued problem (RC). In set-valued optimization, i.e., in optimization with a set-valued objective function, the so called set approach [EJ12, HJ11, Kur98] uses order relations to compare the sets that are the images of the objective function. One widely used order relation is the $u$-type less order relation $\preccurlyeq_{K}$, see [HJ11, Def. 3.2], which is defined for arbitrary nonempty sets $A, B \subseteq \mathbb{R}^{k}$ by

$$
A \preccurlyeq{ }_{K} B: \Leftrightarrow A \subseteq B-K .
$$

The order relation $\preccurlyeq_{K}$ is a reflexive and transitive binary relation. It can also be written in the following form:

$$
A \preccurlyeq K{ }_{K} B \Leftrightarrow(\forall a \in A \exists b \in B: a \leqq b) .
$$

If we replace $K$ by $K \backslash\{0\}$ or int $(K)$ in (2), and thus $\leqq$ by $\leq$ or by $<$ in (3), we analogously obtain the set relations $\preccurlyeq K \backslash\{0\}$ and $\preccurlyeq_{\operatorname{int}(K)}$. However, these are no longer reflexive.

In order to specify optimal solutions of the set-valued optimization problem (RC), we make use of the following definition, see, e.g., [RMS07].

Definition 4. Let a nonempty set $X \subseteq \mathbb{R}^{n}$ and a set-valued map $H: X \rightrightarrows \mathbb{R}^{k}$ be given with $H(x) \neq \emptyset$ for all $x \in X$. The element $x^{*} \in X$ is called strictly optimal solution of the set-valued optimization problem

$$
\min _{x \in X} H(x)
$$

w.r.t. $\preccurlyeq$, where $\preccurlyeq \in\left\{\preccurlyeq_{K}, \preccurlyeq_{\operatorname{int}(K)}, \preccurlyeq K \backslash\{0\}\right\}$, if there exists no $x \in X \backslash\left\{x^{*}\right\}$ with $H(x) \preccurlyeq$ $H\left(x^{*}\right)$.

Using the set relation (2) we can replace

$$
f_{Z}(x) \subseteq f_{Z}\left(x^{*}\right)-[\operatorname{int}(K) / K \backslash\{0\} / K]
$$

in (1) by

$$
f_{Z}(x) \quad\left[\preccurlyeq_{\operatorname{int}(K)} / \preccurlyeq_{K \backslash\{0\}} / \preccurlyeq_{K}\right] \quad f_{Z}\left(x^{*}\right)
$$

as done similarly in $\left[\mathrm{IKK}^{+} 14\right]$ for multi-objective parameter uncertainty. Consequently, we receive equivalence of the optimal solutions to $(\mathrm{RC})$ using Definition 4 and decision robust efficient solutions according to Definition 3.

Theorem 5. A point $x^{*} \in X$ is a decision robust [weakly/·strictly] efficient solution of $(\mathcal{P}(z), z \in Z)$ if and only if $x^{*} \in X$ is a strictly optimal solution of the set-valued optimization problem (RC) w.r.t. $\left[\preccurlyeq_{\operatorname{int}(K)} / \preccurlyeq_{K \backslash\{0\}} / \preccurlyeq_{K}\right]$.

It is known from multiobjective optimization with parameter uncertainty that efficient solutions to the unperturbed deterministic problem are not necessarily robust efficient, see, e.g., [FW14, p. 431]. The following example shows that this is also true for multiobjective optimization with decision uncertainty. 
Example 1. Let

$$
(\mathcal{P}) \quad \min _{x \in \Omega}\left(\begin{array}{c}
x_{1}^{2} \\
-x_{2}
\end{array}\right)
$$

and

$$
(\mathcal{P}(z), z \in Z)=\left(\min _{x+z \in \Omega}\left(\begin{array}{c}
\left(x_{1}+z_{1}\right)^{2} \\
-x_{2}-z_{2}
\end{array}\right), z \in Z\right)
$$

where

and

$$
\begin{aligned}
& \Omega=\operatorname{conv}\left\{\left(\begin{array}{c}
-0.6 \\
0
\end{array}\right),\left(\begin{array}{c}
-0.6 \\
1
\end{array}\right),\left(\begin{array}{l}
1 \\
1
\end{array}\right),\left(\begin{array}{l}
1 \\
0
\end{array}\right),\left(\begin{array}{c}
0.7 \\
-0.3
\end{array}\right),\left(\begin{array}{c}
-0.3 \\
-0.3
\end{array}\right)\right\} \\
& Z=\operatorname{conv}\left(\left\{\left(\begin{array}{c}
-0.6 \\
0
\end{array}\right),\left(\begin{array}{l}
0 \\
0
\end{array}\right),\left(\begin{array}{l}
-0.3 \\
-0.3
\end{array}\right)\right\}\right) .
\end{aligned}
$$

Consequently, we obtain $X=[0,1]^{2}$ as the set of decision robust feasible solutions. The feasible set $\Omega$ and the decision robust feasible set $X$ are visualized in Figure 2.
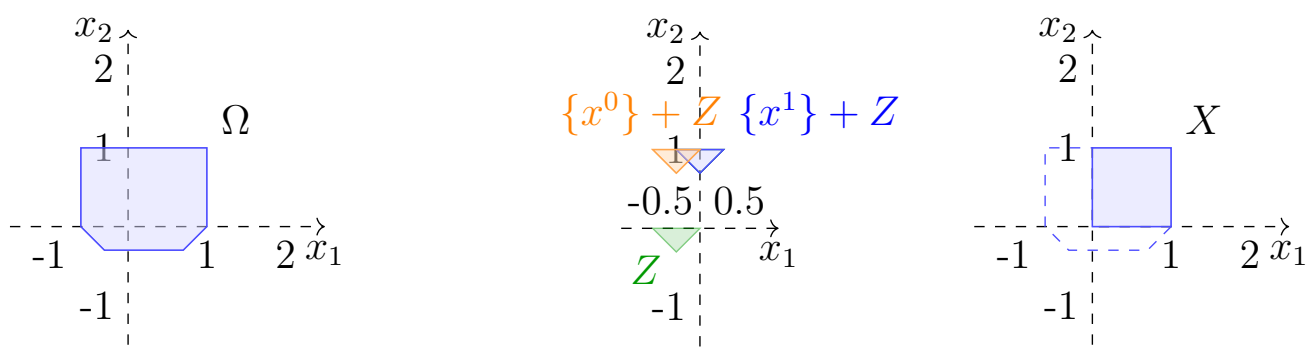

Figure 2: Illustration of the sets $\Omega$ and $X$ in Example 1.

Furthermore, let $K=\mathbb{R}_{+}^{2}$. We obtain

$$
\text { (RC) } \min _{x \in X} f_{Z}(x) \quad \text { where } \quad f_{Z}(x)=\left\{\left(\begin{array}{c}
\left(x_{1}+z_{1}\right)^{2} \\
-x_{2}-z_{2}
\end{array}\right), z \in Z\right\} \text {. }
$$

Consider $x^{0}:=\left(\begin{array}{c}0.3 \\ 1\end{array}\right)$ and $x^{1}:=\left(\begin{array}{l}0 \\ 1\end{array}\right)$. In this example:

- The solution $x^{1}$ is efficient for $(\mathcal{P})$, but not decision robust efficient for $(\mathcal{P}(z), z \in Z)$.

- The solution $x^{0}$ is decision robust efficient for $(\mathcal{P}(z), z \in Z)$, but not efficient for $(\mathcal{P})$.

One can check that there exists no $x \in \Omega$ with $f(x) \in\left\{f\left(x^{1}\right)\right\}-\mathbb{R}_{+}^{2} \backslash\{0\}$. Hence, $x^{1}$ is efficient for the deterministic problem. In particular,

$$
f\left(x^{1}\right)=\left(\begin{array}{c}
0 \\
-1
\end{array}\right) \leq\left(\begin{array}{c}
0.09 \\
-1
\end{array}\right)=f\left(x^{0}\right) .
$$

Consequently, $x^{0}$ is not efficient for the deterministic problem. Looking at the set $f_{Z}(x)$ for each $x \in X$, one can check that there is no $x \in X \backslash\left\{x^{0}\right\}$ with $f_{Z}(x) \subseteq f_{Z}\left(x^{0}\right)-\mathbb{R}_{+}^{2} \backslash\{0\}$ and $x^{0}$ is decision robust efficient according to Definition 3. In particular, it holds $f_{Z}\left(x^{0}\right) \subseteq$ $f_{Z}\left(x^{1}\right)-\mathbb{R}_{+}^{2} \backslash\{0\}$ as illustrated in Figure 3. Hence, $x^{1}$ is not decision robust efficient even though being efficient for the deterministic problem. 

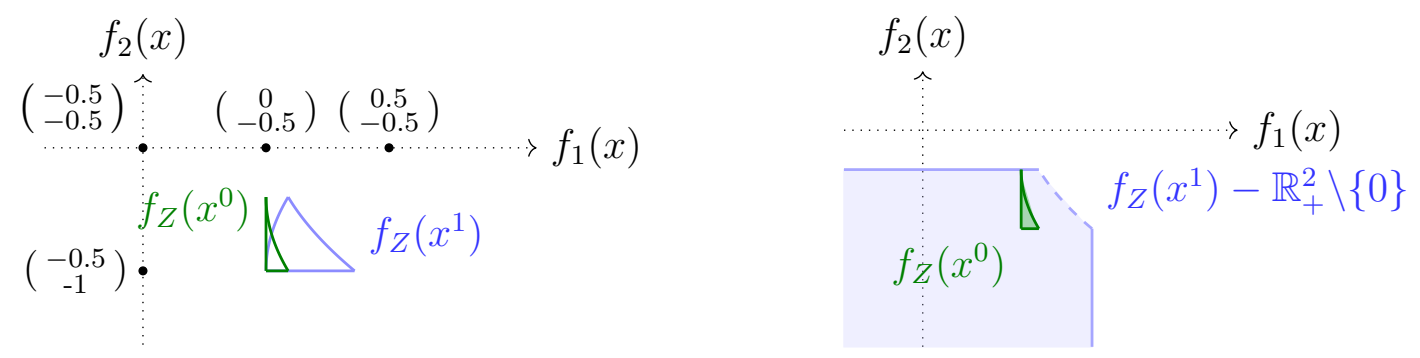

Figure 3: Illustration of the set $f_{Z}\left(x^{0}\right)$ and the set $f_{Z}\left(x^{1}\right)$ of Example 1.

\subsection{Approach using Supremal Sets}

In minmax robust optimization, solutions are determined that perform best in the objective function when evaluated together with worst-case realizations of uncertainty. In view of that, we investigate suprema of the sets $f_{Z}(x)$ for $x \in X$. We show that minimizing the supremal sets of the sets $f_{Z}$ is equivalent to solving (RC).

We use a concept for the supremum of a set that is defined and studied thoroughly in [Nie80] and [Löh11].

Definition 6. For a bounded nonempty subset $A \subsetneq \mathbb{R}^{k}$ the supremal set of $A$ is defined as

$$
\operatorname{Sup}(A)=\{y \in \operatorname{cl}(A-K) \mid(\{y\}+\operatorname{int}(K)) \cap \operatorname{cl}(A-K)=\emptyset\} .
$$

According to [Löh11, Cor. 1.48, Cor. 1.49], for a bounded nonempty subset $A \subsetneq \mathbb{R}^{k}$ it holds

$$
\operatorname{Sup}(A)=\operatorname{bd}(\operatorname{Sup}(A)-K) .
$$

For an illustration of a supremal set, we refer to the right hand picture of Figure 3 . The boundary of the set $f_{Z}\left(x^{1}\right)-\mathbb{R}_{+}^{2} \backslash\{0\}$ (including the dashed and solid lines) is exactly the supremal set of $f_{Z}\left(x^{1}\right)$ in Example 1 .

Following Definition 6, we formulate another set-valued optimization problem

$$
\min _{x \in X} f_{\text {Sup }}(x) \quad \text { with } \quad f_{\text {Sup }}: X \rightrightarrows \mathbb{R}^{k}, x \mapsto \operatorname{Sup}\left(f_{Z}(x)\right)
$$

In general set-valued optimization problems, the image set of the objective function, such as $f_{\text {Sup }}(x)$ in $(5)$, is assumed to be nonempty. To this end, we additionally assume $f$ to be continuous in the remainder of the section.

Remark 1. Due to our assumptions, the perturbation set $Z$ is nonempty and compact and $f: \Omega \rightarrow \mathbb{R}^{k}$ is continuous. Hence, $f_{Z}(x)$ is compact and nonempty for all $x \in X \subseteq \Omega$ and

$$
f_{Z}(x)-K=\operatorname{cl}\left(f_{Z}(x)-K\right) \notin\left\{\mathbb{R}^{k}, \emptyset\right\} .
$$

According to Corollary 1.44 in [Löh11], this directly implies

$$
f_{\text {Sup }}(x)=\operatorname{Sup}\left(f_{Z}(x)\right) \neq \emptyset \text {. }
$$


The following lemma is fundamental for our main result which relates the solutions of (5) to the solutions of $(\mathrm{RC})$ and the decision robust [weakly/./strictly] efficient solutions of $(\mathcal{P}(z), z \in Z)$.

Lemma 7. Let $A, B \subsetneq \mathbb{R}^{k}$ be compact sets. Then

$$
A-[\operatorname{int}(K) / K \backslash\{0\} / K]=\operatorname{Sup}(A)-[\operatorname{int}(K) / K \backslash\{0\} / K]
$$

and thus for any $\preccurlyeq \in\left\{\preccurlyeq_{K}, \preccurlyeq_{\operatorname{int}(K)}, \preccurlyeq K \backslash\{0\}\right\}$ it holds

$$
A \preccurlyeq B \quad \Leftrightarrow \quad \operatorname{Sup}(A) \preccurlyeq \operatorname{Sup}(B) .
$$

Proof. In [Löh11, Chapter 1] it was shown that $\operatorname{Sup}(A)-\operatorname{int}(K)=\operatorname{cl}(A-K)-\operatorname{int}(K)$. As $A$ is compact and $K$ is closed we have $\operatorname{cl}(A-K)=A-K$ and we get $\operatorname{Sup}(A)-\operatorname{int}(K)=$ $A-\operatorname{int}(K)$. Next we show

$$
A-K=\operatorname{Sup}(A)-K \text {. }
$$

By [Löh11, p.24 Cor. 1.48 (ix)] we have $\operatorname{cl}(A-K)=\operatorname{Sup}(A) \cup(\operatorname{Sup}(A)-\operatorname{int}(K))$. And thus

$$
A-K=\operatorname{cl}(A-K)=\operatorname{Sup}(A) \cup(\operatorname{Sup}(A)-\operatorname{int}(K)) \subseteq \operatorname{Sup}(A)-K .
$$

By the definition of the supremal set and the compactness of $A$ we also have $\operatorname{Sup}(A)-K \subseteq$ $\operatorname{cl}(A-K)-K=A-K$ and we have shown (6). Finally we get from (6)

$$
A-K \backslash\{0\}=A-K-K \backslash\{0\}=\operatorname{Sup}(A)-K-K \backslash\{0\}=\operatorname{Sup}(A)-K \backslash\{0\} .
$$

Due to Relation (2) we obtain the last assertion:

$$
\begin{aligned}
A \subseteq B-[\operatorname{int}(K) / K \backslash\{0\} / K] & \Leftrightarrow A-K \subseteq B-[\operatorname{int}(K) / K \backslash\{0\} / K] \\
& \Leftrightarrow \operatorname{Sup}(A)-K \subseteq \operatorname{Sup}(B)-[\operatorname{int}(K) / K \backslash\{0\} / K] \\
& \Leftrightarrow \operatorname{Sup}(A) \subseteq \operatorname{Sup}(B)-[\operatorname{int}(K) / K \backslash\{0\} / K] .
\end{aligned}
$$

Due to $Z$ being compact, the set $f_{Z}(x)$ is compact for a continuous objective function $f$ and for each $x \in X$.

Corollary 8. Let $f$ be continuous. Then it holds for all $x, x^{\prime} \in X$ and $\preccurlyeq \in\left\{\preccurlyeq_{K}, \preccurlyeq_{\operatorname{int}(K)}, \preccurlyeq K \backslash\{0\}\right\}$

$$
f_{Z}(x) \preccurlyeq f_{Z}\left(x^{\prime}\right) \quad \text { if and only if } \operatorname{Sup}\left(f_{Z}(x)\right) \preccurlyeq \operatorname{Sup}\left(f_{Z}\left(x^{\prime}\right)\right) \text {, }
$$

i.e., the set-valued optimization problems ( $\mathrm{RC})$ and (5) are equivalent in the sense that the sets of strictly optimal solutions w.r.t. $\left[\preccurlyeq_{\operatorname{int}(K)} / \preccurlyeq_{K \backslash\{0\}} / \preccurlyeq_{K}\right]$ are the same.

Using Theorem 5 and Corollary 8 we directly obtain our main result of this section.

Theorem 9. Let $f$ be continuous. A point $x^{*} \in X$ is a decision robust [weakly/·strictly] efficient solution of $(\mathcal{P}(z), z \in Z)$ if and only if $x^{*} \in X$ is a strictly optimal solution for the set-valued optimization problem (5) w.r.t. $\left[\preccurlyeq_{\operatorname{int}(K)} / \preccurlyeq_{K \backslash\{0\}} / \preccurlyeq_{K}\right]$. 


\section{Properties of the Set-Valued Functions $f_{Z}(\cdot)$ and $\operatorname{Sup}\left(f_{Z}(\cdot)\right)$}

We prove that convexity of the vector-valued function $f$ implies convexity of the set-valued map $f_{Z}$. Furthermore, we prove that continuity of the vector-valued function $f$ leads to lower and upper semicontinuity of the set-valued map $f_{Z}$, which maps a point to the image set of all of its realizations. Also, if $f$ is continuous, the set-valued map $f_{\text {Sup }}$, which maps every solution $x \in X$ to the supremal set of the set $f_{Z}(x)$, is lower semicontinuous.

\subsection{Convexity}

We start by recalling the definition of a $K$-convex single-valued and a $K$-convex set-valued map for a convex cone $K$. See here, e.g., [Kur96, Def. 2.1],[AF90, Lemma 2.1.2] as well as [BP03] and the references therein.

Definition 10. Let $Y \subseteq \mathbb{R}^{n}$ be a convex set. A map $f: Y \rightarrow \mathbb{R}^{k}$ is called $K$-convex, if for all $x^{1}, x^{2} \in Y$ and $\lambda \in[0,1]$ it holds that $\lambda f\left(x^{1}\right)+(1-\lambda) f\left(x^{2}\right) \in\left\{f\left(\lambda x^{1}+(1-\lambda) x^{2}\right)\right\}+K$.

Definition 11. Let $Y \subseteq \mathbb{R}^{n}$ be a convex set. A set-valued map $H: Y \rightrightarrows \mathbb{R}^{k}$ is called $K$-convex, if for all $x^{1}, x^{2} \in Y$ and $\lambda \in[0,1]$ it holds that $\lambda H\left(x^{1}\right)+(1-\lambda) H\left(x^{2}\right) \subseteq$ $H\left(\lambda x^{1}+(1-\lambda) x^{2}\right)+K$.

$K$-convexity plays an important role for many tools used in set optimization such as subdifferentials, derivatives and optimality conditions. Under convexity assumptions in $\Omega$, $Z$ and $f$ we obtain cone-convexity of our set-valued objective map.

Proposition 12. Let $\Omega$ and $Z$ be convex. Then, $X$ is a convex set. If, in addition, $f: \Omega \rightarrow \mathbb{R}^{k}$ is a $K$-convex single-valued map, then $f_{Z}: X \rightrightarrows \mathbb{R}^{k}$ is a $K$-convex set-valued map.

Proof. For fixed $z \in Z$, the set $\left\{x \in \mathbb{R}^{n} \mid x+z \in \Omega\right\}$ is convex. Hence, $X=\bigcap_{z \in Z}\{x \in$ $\left.\mathbb{R}^{n} \mid x+z \in \Omega\right\}$ is convex as an intersection of convex sets.

Let $a \in f_{Z}\left(x^{1}\right)$ and $b \in f_{Z}\left(x^{2}\right)$ be arbitrarily chosen. Then there exist $z^{1}, z^{2} \in Z$ with $a=f\left(x^{1}+z^{1}\right)$ and $b=f\left(x^{2}+z^{2}\right)$. With $x:=\lambda x^{1}+(1-\lambda) x^{2}$ and $z:=\lambda z^{1}+(1-\lambda) z^{2} \in Z$ we obtain

$$
\begin{aligned}
\lambda a+(1-\lambda) b & =\lambda f\left(x^{1}+z^{1}\right)+(1-\lambda) f\left(x^{2}+z^{2}\right) \\
& \in\left\{f\left(\lambda\left(x^{1}+z^{1}\right)+(1-\lambda)\left(x^{2}+z^{2}\right)\right)\right\}+K \\
& =\{f(x+z)\}+K \\
& \subseteq f_{Z}(x)+K .
\end{aligned}
$$

Note that $\operatorname{Sup}\left(f_{Z}(\cdot)\right)$ is not a $K$-convex map and that, in contrast to $K$-convexity, linearity of a function $f: \Omega \rightarrow \mathbb{R}^{k}$ does not necessarily lead to linearity in the corresponding set-valued function $f_{Z}: X \rightrightarrows \mathbb{R}^{k}$. 


\subsection{Semicontinuity}

(Semi)continuity is a very important property in optimization as it is a basic assumption for a large class of algorithms such as decent methods (see, e.g., [Jah15]). Hence, we investigate semicontinuity as a first basic property of the new problem on multiobjective decision uncertainty. We use here the standard definition as provided in Definition 3.1.1 in [KTZ15], see also [AF90, Section 1.4].

We prove that, if $f$ is continuous everywhere, the set-valued functions $f_{Z}$ and $\operatorname{Sup}\left(f_{Z}(\cdot)\right)$ inherit continuity properties. We show that continuity of $f$ leads to semicontinuity of $f_{Z}$. Moreover, $\operatorname{Sup}\left(f_{Z}(\cdot)\right)$ is lower but not necessarily upper semicontinuous if $f$ is continuous.

Throughout this section, we consider the feasible set $\Omega \subseteq \mathbb{R}^{n}$ as a metric space. $\Omega$ is equipped with the metric derived from an arbitrary norm $\|\cdot\|$ on $\mathbb{R}^{n}$. Hence, we can use the following definition of [AF90, Section 1.4] to define semicontinuity on subsets of $\mathbb{R}^{n}$, such as $\Omega$.

Definition 13. Let $S$ be a metric space and let $H: S \rightrightarrows \mathbb{R}^{k}$ be a set-valued map.

(a) $H$ is called lower semicontinuous at $x^{0} \in S$, if for all open sets $V \subseteq \mathbb{R}^{k}$ with $H\left(x^{0}\right) \cap$ $V \neq \emptyset$ there is a neighborhood $U$ of $x^{0}$ such that $H(x) \cap V \neq \emptyset$ for all $x \in U$. $H$ is lower semicontinuous if it is lower semicontinuous at any $x^{0} \in S$.

(b) $H$ is called upper semicontinuous at $x^{0} \in S$ if for all open sets $V \subseteq \mathbb{R}^{k}$ with $H\left(x^{0}\right) \subseteq$ $V$ there is a neighborhood $U$ of $x^{0}$ such that $H(x) \subseteq V$ for all $x \in U$. $H$ is upper semicontinuous if it is upper semicontinuous at any $x^{0} \in S$.

An equivalent definition can be found in [KTZ15] under the name of lower and upper continuity. For a survey of the different and related notions for upper and lower semicontinuity used in the literature, we refer to [DD79].

If the function $f$ is not continuous everywhere, the set-valued map $f_{Z}$ might be neither lower nor upper semicontinuous. By a similar example, it is also easy to see that the set-valued map $f_{Z}$ does not need to be upper semicontinuous if $f$ is continuous everywhere except at one point.

Example 2. Let $f: \mathbb{R} \rightarrow \mathbb{R}$ be defined by

$$
f(x)= \begin{cases}0 & \text { if } x \geq-1 \\ 1 & \text { else }\end{cases}
$$

and $Z:=[-1,1]$. Then

$$
f_{Z}(x)= \begin{cases}\{0\} & \text { if } x \geq 0 \\ \{0,1\} & \text { if }-2 \leq x<0 \\ \{1\} & \text { if } x<-2\end{cases}
$$

Let $x^{0}=0$ and $V=(-0.5,0.5)$. Then $f_{Z}\left(x^{0}\right) \subseteq V$ but there is no neighborhood $U$ of $x^{0}$ such that $f_{Z}(x) \subset V$ for all $x \in U$. However, $f_{Z}$ is lower semicontinuous at $x^{0}$. 
Next, we show that $f_{Z}$ is both upper and lower semicontinuous if $f$ is continuous.

Proposition 14. Let $f: \Omega \rightarrow \mathbb{R}^{k}$ be continuous. Then $f_{Z}: X \rightrightarrows \mathbb{R}^{k}$ is lower semicontinuous.

Proof. Let $x^{0} \in X$ and let $V \subseteq \mathbb{R}^{k}$ be an arbitrary open set such that $f_{Z}\left(x^{0}\right) \cap V \neq \emptyset$. Then there exists $z \in Z$ such that $f\left(x^{0}+z\right) \in V$. As $V$ is an open set, there exists $\varepsilon>0$ such that $B\left(f\left(x^{0}+z\right), \varepsilon\right) \subseteq V$. Because $f$ is continuous in $x^{0}+z$, there exists $\delta>0$ such that $\left\|f\left(x^{0}+z\right)-f(w)\right\|<\varepsilon$ for all $w \in \Omega$ with $\left\|x^{0}+z-w\right\|<\delta$. We define the neighborhood $U\left(x^{0}\right)=\left\{x \in X \mid\left\|x-x^{0}\right\|<\delta\right\}$ of $x^{0}$ in $X$. Then we have for all $x \in U\left(x^{0}\right)$ an element $w:=x+z \in\{x\}+Z$ and

$$
\left\|x^{0}+z-w\right\|=\left\|x-x^{0}\right\|<\delta .
$$

Hence, $f(x+z)=f(w) \in B\left(f\left(x^{0}+z\right), \varepsilon\right) \subseteq V$ and $f_{Z}(x) \cap V \neq \emptyset$.

Proposition 15. Let $f: \Omega \rightarrow \mathbb{R}^{k}$ be continuous. Then $f_{Z}: X \rightrightarrows \mathbb{R}^{k}$ is upper semicontinuous.

Proof. Let $x^{0} \in X$ be given and let $V \subseteq \mathbb{R}^{k}$ be open such that $f_{Z}\left(x^{0}\right) \subseteq V$. Because $f$ is continuous, the inverse image $W:=\{y \in \Omega \mid f(y) \in V\}$ is open in $\Omega$. If $W=\Omega$, we immediately obtain $\{x\}+Z \subseteq W$ and $f_{Z}(x) \subseteq V$ for all $x \in X$ and our claim is true.

Otherwise, $A:=\Omega \backslash W$ is nonempty and closed in the metric space $\Omega$ and disjoint from the compact set $\left\{x^{0}\right\}+Z \subseteq W$. Consequently, there exists $\delta>0$ such that for all $y \in\left\{x^{0}\right\}+Z$ and $a \in A$

$$
\|y-a\|>\delta
$$

Thus,

$$
\bigcup_{z \in Z} B\left(x^{0}+z, \delta\right) \subseteq W
$$

We define the neighborhood $U:=B\left(x^{0}, \delta\right) \subseteq X$. For all $x \in U$ and $z \in Z$ we have $\left\|x+z-\left(x^{0}+z\right)\right\|=\left\|x-x^{0}\right\|<\delta$ and

$$
\{x\}+Z \subseteq \bigcup_{z \in Z} B\left(x^{0}+z, \delta\right) \subseteq W
$$

where $B\left(x^{0}+z, \delta\right)$ denotes an open ball in the metric space $\Omega$ for all $z \in Z$. Hence, for all $x \in U$

$$
f_{Z}(x) \subseteq\{f(w) \mid w \in W\} \subseteq V
$$

As we have seen in Theorem 9, determining whether a decision robust feasible solution is decision robust efficient is equivalent to a comparison of supremal sets. Thus, we continue by examining semicontinuity properties of the set-valued function

$$
f_{\text {Sup }}: X \rightrightarrows \mathbb{R}^{k}, \quad x \mapsto \operatorname{Sup}\left(f_{Z}(x)\right)
$$


that was already introduced as (5) in Section 2.3.

The following lemma serves as a preparation for proving the lower semicontinuity of the function $f_{\text {Sup }}$ in Proposition 17.

Lemma 16. Let $\varepsilon>0$, and let $a \in \operatorname{int}(K)$ with $\|a\|<\varepsilon$. Then

$$
T=B(0, \varepsilon) \cap(\{a\}-\operatorname{int}(K)) \cap(\{-a\}+\operatorname{int}(K))
$$

(a) is convex and open,

(b) $B(0, \tilde{\varepsilon}) \subseteq T \subseteq B(0, \varepsilon)$ for some $0<\tilde{\varepsilon}<\varepsilon$,

(c) $T$ is symmetric, i.e. $T=-T$.

(d) For all $s, u \in \mathbb{R}^{k}$ it holds

$$
\{s\}+T \subseteq\{u\}-\operatorname{int}(K) \quad \Leftrightarrow \quad\{u\}+T \subseteq\{s\}+\operatorname{int}(K) .
$$

(e) $a \in \operatorname{cl}(T)$.

Proof. The set $T$ is convex and open as an intersection of three convex and open sets, hence (a) holds. Furthermore, $T \subseteq B(0, \varepsilon)$. To see the second part of (b), note that $0 \in T$ because $0 \in B(0, \varepsilon), 0=a-a \in\{a\}-\operatorname{int}(K)$ and $0=-a+a \in\{-a\}+\operatorname{int}(K)$. Because $T$ is open, there exists $\tilde{\varepsilon}>0$ such that $B(0, \tilde{\varepsilon}) \subseteq T$.

Next, (c) results from $B(0, \varepsilon)=-B(0, \varepsilon)$ and from $\{a\}-\operatorname{int}(K)=-(\{-a\}+\operatorname{int}(K))$, and $(\mathrm{d})$ is a direct consequence of (c). Finally, for (e), note that $a \in \operatorname{int}(B(0, \varepsilon))$ and that $a \in \operatorname{int}(\{-a\}+\operatorname{int}(K))$ since $a=-a+2 \cdot a$ and $a \in \operatorname{int}(K)$. Because $a$ is in the interior of both sets, every sequence that is convergent to $a$ has a subsequence that converges to $a$ in $B(0, \varepsilon) \cap(\{-a\}+\operatorname{int}(K))$. Furthermore, $a \in \operatorname{cl}(\{a\}-\operatorname{int}(K))$ and the result follows.

Proposition 17. Let $f: \Omega \rightarrow \mathbb{R}^{k}$ be continuous. Then $f_{\text {Sup }}: X \rightrightarrows \mathbb{R}^{k}$ with $x \mapsto \operatorname{Sup}\left(f_{Z}(x)\right)$ is lower semicontinuous.

Proof. Let $x^{0} \in X$ and let $V \subseteq \mathbb{R}^{k}$ be an open set such that $\operatorname{Sup}\left(f_{Z}\left(x^{0}\right)\right) \cap V \neq \emptyset$. Choose $s \in \operatorname{Sup}\left(f_{Z}\left(x^{0}\right)\right) \cap V \neq \emptyset$. Due to Lemma 7 we have for all $x \in X$

$$
f_{Z}(x)-K=\operatorname{Sup}\left(f_{Z}(x)\right)-K
$$

Consequently, there exist $z \in Z$ and $k \in K$ such that $s:=f\left(x^{0}+z\right)-k$. Furthermore, there exists $\varepsilon>0$ such that $\{s\}+B(0, \varepsilon) \subseteq V$.

Additionally, there exists a convex neighborhood $T$ of $0 \in \mathbb{R}^{k}$ that satisfies the properties of Lemma 16. In accordance with Property (a) of Lemma 16, let $a \in \mathbb{R}^{k}$ such that

$$
T=B(0, \varepsilon) \cap(\{a\}-\operatorname{int}(K)) \cap(\{-a\}+\operatorname{int}(K)) .
$$


According to Property (b) of Lemma 16, $\{s\}+T$ is a convex neighborhood of $s$ satisfying $\{s\}+B(0, \tilde{\varepsilon}) \subseteq\{s\}+T \subseteq\{s\}+B(0, \varepsilon) \subseteq V$. Because $f$ is continuous, there exists for $\tilde{\varepsilon}>0$ some $\delta_{0}>0$ such that

$$
f(y) \in\left\{f\left(x^{0}+z\right)\right\}+T \text { for all } y \in \Omega \text { with }\left\|y-\left(x^{0}+z\right)\right\|<\delta_{0} .
$$

Furthermore, $f_{Z}$ is upper semicontinuous in $x^{0}$ due to Proposition 15. Consequently, there exists $\delta_{1}>0$ such that $f_{Z}(x) \subseteq f_{Z}\left(x^{0}\right)+T$ for all $x \in X$ with $\left\|x-x^{0}\right\|<\delta_{1}$. We define $\delta:=\min \left\{\delta_{0}, \delta_{1}\right\}$. The set $U:=\left\{x \in X \mid\left\|x-x^{0}\right\|<\delta\right\}$ is a neighborhood of $x^{0}$ in $X$ with respect to the subset topology inherited from $\mathbb{R}^{n}$.

Assume that there exists $x \in U$ such that

$$
\operatorname{Sup}\left(f_{Z}(x)\right) \cap(\{s\}+T)=\emptyset .
$$

Next, we show that this assumption leads to

$$
\{s\}+T \subseteq \operatorname{Sup}\left(f_{Z}(x)\right)-K .
$$

We have $\left\|x+z-\left(x^{0}+z\right)\right\|<\delta \leq \delta_{0}$, hence, using (9) and the definition of $s$, we get

$$
f(x+z)-k \in\{s\}+T \text {. }
$$

Together with (7), we obtain

$$
f(x+z)-k \in(\{s\}+T) \cap\left(f_{Z}(x)-K\right)=(\{s\}+T) \cap\left(\operatorname{Sup}\left(f_{Z}(x)\right)-K\right) .
$$

By our Assumption (10), $f(x+z)-k \notin \operatorname{Sup}\left(f_{Z}(x)\right)$. Next, we assume that (11) does not hold. Then, there exists $t \in T$ such that $s+t \notin \operatorname{Sup}\left(f_{Z}(x)\right)-K$. Due to (12) and because of $\{s\}+T$ being convex due to Lemma 16, there exists $w \in \operatorname{conv}\{f(x+z)-k, s+t\}$ such that, using (4),

$$
w \in \operatorname{bd}\left(\operatorname{Sup}\left(f_{Z}(x)\right)-K\right) \cap(\{s\}+T)=\operatorname{Sup}\left(f_{Z}(x)\right) \cap(\{s\}+T)
$$

in contradiction to Assumption (10). Hence, (11) holds and by using (7) and Remark 1 we obtain

$$
\operatorname{cl}(\{s\}+T) \subseteq \operatorname{cl}\left(\operatorname{Sup}\left(f_{Z}(x)\right)-K\right)=\operatorname{cl}\left(f_{Z}(x)-K\right)=f_{Z}(x)-K .
$$

By Property (e) in Lemma 16 we get

$$
s+a \in \mathrm{cl}(\{s\}+T) \subseteq f_{Z}(x)-K .
$$

Therefore, there exists $\tilde{z} \in Z$ such that $s+a \in\{f(x+\tilde{z})\}-K$. Consequently, due to (8),

$$
\{s\}+T \subseteq\{s+a\}-\operatorname{int}(K) \subseteq\{f(x+\tilde{z})\}-\operatorname{int}(K) .
$$

According to Property (d) in Lemma 16 this is equivalent to

$$
\{f(x+\tilde{z})\}+T \subseteq\{s\}+\operatorname{int}(K) .
$$


Because of $\left\|x-x^{0}\right\|<\delta \leq \delta_{1}$, there exists $z^{0} \in Z$ such that $f\left(x^{0}+z^{0}\right) \in\{f(x+\tilde{z})\}+T$. As a consequence, we finally obtain

$$
f\left(x^{0}+z^{0}\right) \in\{f(x+\tilde{z})\}+T \subseteq\{s\}+\operatorname{int}(K)
$$

in contradiction to $s \in \operatorname{Sup}\left(f_{Z}\left(x^{0}\right)\right)$. Thus, (10) does not hold and we have for all $x \in U$

$$
\emptyset \neq \operatorname{Sup}\left(f_{Z}(x)\right) \cap(\{s\}+T) \subseteq \operatorname{Sup}\left(f_{Z}(x)\right) \cap(\{s\}+B(0, \varepsilon)) \subseteq \operatorname{Sup}\left(f_{Z}(x)\right) \cap V .
$$

While the set-valued function $f_{\text {Sup }}(\cdot)=\operatorname{Sup}\left(f_{Z}(\cdot)\right)$ is lower semicontinuous, it is not upper semicontinuous as the following counterexample shows.

Example 3. Let $\Omega=[-1,1]^{2}, Z=\{0\}, K=\mathbb{R}_{+}^{2}$ and $f=\mathrm{id}: \mathbb{R}^{2} \rightarrow \mathbb{R}^{2}, \quad x \mapsto x$. Then $X=\Omega$ and we have for all $x \in X$

$$
\operatorname{Sup}\left(f_{Z}(x)\right)=\{x\}-\operatorname{bd}\left(\mathbb{R}_{+}^{2}\right) .
$$

The set

$$
V:=\left\{z \in \mathbb{R}^{2} \mid z_{1} \leq 0\right\} \cup\left\{z \in \mathbb{R}^{2} \mid z_{1}>0, z_{2}>-\frac{1}{z_{1}}\right\}
$$

is a neighborhood of $\operatorname{Sup}\left(f_{Z}\left(x^{0}\right)\right)$ with $x^{0}=(0,0)^{\top}$. We show that for each $\delta>0$ there exists $x \in X$ with $\left\|x^{0}-x\right\|_{\infty}<\delta$ and with $\operatorname{Sup}\left(f_{Z}(x)\right) \nsubseteq V$. Choose an arbitrary $\delta>0$ with $\delta<2$. Then $x^{\prime}:=\frac{\delta}{2}(1,0)^{\top} \in X$ satisfies $\left\|x^{0}-x^{\prime}\right\|_{\infty}<\delta$. We have $w:=(\delta / 2,-4 / \delta)^{\top} \in$ $\operatorname{Sup}\left(f_{Z}\left(x^{\prime}\right)\right)$ but due to

$$
w_{2}=-\frac{4}{\delta} \leq-\frac{2}{\delta}=-\frac{1}{w_{1}}
$$

it holds $w \notin V$. Because $\delta>0$ can be chosen arbitrarily small, we see that $\operatorname{Sup}\left(f_{Z}(\cdot)\right)$ is not upper semicontinuous.

The results on the semicontinuity of the functions considered in this section are summarized in the following table. For continuous objective functions $f$ we have:

\begin{tabular}{l||c|c|} 
& $f_{Z}(\cdot)$ & $\operatorname{Sup}\left(f_{Z}(\cdot)\right)$ \\
\hline \hline lower semicontinuous & $\checkmark$ & $\boldsymbol{\checkmark}$ \\
\hline upper semicontinuous & $\checkmark$ & $\boldsymbol{X}$
\end{tabular}

Even though $\operatorname{Sup}\left(f_{Z}(\cdot)\right)$ is not upper semicontinuous, we finally show that $\operatorname{Sup}\left(f_{Z}\left(x^{0}\right)\right)$ and $\operatorname{Sup}\left(f_{Z}(x)\right)$ are arbitrary close for $x^{0}, x \in X$ with $\left\|x^{0}-x\right\|<\delta$ if we chose $\delta>0$ small enough.

Theorem 18. Let $f: \Omega \rightarrow \mathbb{R}^{k}$ be continuous. Then for each $x^{0} \in X$ and $\varepsilon>0$ there exists $\delta>0$ such that

$$
\operatorname{Sup}\left(f_{Z}(x)\right) \subseteq \operatorname{Sup}\left(f_{Z}\left(x^{0}\right)\right)+B(0, \varepsilon)
$$

for all $x \in X$ with $\left\|x^{0}-x\right\|<\delta$. 
Proof. Let $x^{0} \in X$ and let $\varepsilon>0$. Let $a \in \operatorname{int}(K)$ such that $\|a\|<\varepsilon$ and define

$$
T=B(0, \varepsilon) \cap(\{a\}-\operatorname{int}(K)) \cap(\{-a\}+\operatorname{int}(K))
$$

as in Lemma 16.

According to Property (b) of Lemma 16, for every $v \in \mathbb{R}^{k}$ the set $\{v\}+T$ is a convex neighborhood of $v$ satisfying $\{v\}+T \subseteq B(v, \varepsilon)$.

Because $f_{Z}\left(x^{0}\right)$ is compact in $\mathbb{R}^{k}$ and $\bigcup_{z \in Z}\left\{f\left(x^{0}+z\right)\right\}+\frac{1}{2} T$ is an open cover of $f_{Z}\left(x^{0}\right)$, there exists a finite subcover

$$
f_{Z}\left(x^{0}\right) \subseteq \bigcup_{\tilde{z} \in \tilde{Z}}\left\{f\left(x^{0}+\tilde{z}\right)\right\}+\frac{1}{2} T,
$$

which will be indexed by $\tilde{Z} \subseteq Z$ with $|\tilde{Z}|<\infty$. Furthermore, there exists $\delta_{1}>0$ such that

$$
f_{Z}(x) \subseteq \bigcup_{\tilde{z} \in \tilde{Z}}\left\{f\left(x^{0}+\tilde{z}\right)\right\}+\frac{1}{2} T
$$

for all $x \in X$ with $\left\|x-x^{0}\right\|<\delta_{1}$ because $f_{Z}$ is upper semicontinuous due to Proposition 15 .

Because $f$ is continuous on $\Omega$, there exists $\delta(\tilde{z})>0$ for each $\tilde{z} \in \tilde{Z}$ such that

$$
f(y) \in\left\{f\left(x^{0}+\tilde{z}\right)\right\}+\frac{1}{2} T \quad \text { for all } \quad y \in \Omega \quad \text { with } \quad\left\|y-\left(x^{0}+\tilde{z}\right)\right\|<\delta(\tilde{z}) .
$$

Let $\delta_{2}=\min \{\delta(\tilde{z}) \mid \tilde{z} \in \tilde{Z}\}$ and define $\delta:=\min \left\{\delta_{1}, \delta_{2}\right\}$.

We hence have that $f(x+\tilde{z}) \in\left\{f\left(x^{0}+\tilde{z}\right)\right\}+\frac{1}{2} T$ for all $x \in X$ with $\left\|x^{0}-x\right\|<\delta \leq \delta_{2}$ and all $\tilde{z} \in \tilde{Z}$. Consequently, $f\left(x^{0}+\tilde{z}\right) \in\{f(x+\tilde{z})\}-\frac{1}{2} T$ and, because of the symmetry of $T$ according to Property (c) in Lemma 16,

$$
f\left(x^{0}+\tilde{z}\right) \in\{f(x+\tilde{z})\}+\frac{1}{2} T .
$$

Additionally, for each $z \in Z$ there exists $\tilde{z} \in \tilde{Z}$ such that

$$
f\left(x^{0}+z\right) \in\left\{f\left(x^{0}+\tilde{z}\right)\right\}+\frac{1}{2} T
$$

because our finite subcover is indexed by $\tilde{Z}$. Together with Relation (16) we see that for each $x \in X$ with $\left\|x^{0}-x\right\|<\delta$ and $z \in Z$ there exists $\tilde{z} \in \tilde{Z}$ such that

$$
f\left(x^{0}+z\right) \in\left\{f\left(x^{0}+\tilde{z}\right)\right\}+\frac{1}{2} T \subseteq\left(\{f(x+\tilde{z})\}+\frac{1}{2} T\right)+\frac{1}{2} T=\{f(x+\tilde{z})\}+T
$$

because $T$ is convex. Hence, using again the symmetry of $T$, we obtain for each $z \in Z$ the existence of some $\tilde{z} \in \tilde{Z}$ such that

$$
f(x+\tilde{z}) \in\left\{f\left(x^{0}+z\right)\right\}+T .
$$


In order to prove Theorem 18, we assume on the contrary that there exists $x \in X$ with $\left\|x^{0}-x\right\|<\delta$ such that

$$
\operatorname{Sup}\left(f_{Z}(x)\right) \nsubseteq \operatorname{Sup}\left(f_{Z}\left(x^{0}\right)\right)+T .
$$

Then there exists $y \in \operatorname{Sup}\left(f_{Z}(x)\right)$ such that $y \notin \operatorname{Sup}\left(f_{Z}\left(x^{0}\right)\right)+T$ and, due to the symmetry of $T$,

$$
(\{y\}+T) \cap \operatorname{Sup}\left(f_{Z}\left(x^{0}\right)\right)=\emptyset .
$$

Recall from Lemma 7 that for all $x^{\prime} \in X$

$$
f_{Z}\left(x^{\prime}\right)-K=\operatorname{Sup}\left(f_{Z}\left(x^{\prime}\right)\right)-K
$$

From (20) we know that there exist $z \in Z$ and $k \in K$ such that

$$
y=f(x+z)-k .
$$

Next, we show that Assumption (19) leads to

$$
\{y\}+T \subseteq \operatorname{Sup}\left(f_{Z}\left(x^{0}\right)\right)-K .
$$

We have $\left\|x-x^{0}\right\|<\delta \leq \delta_{1}$. Due to (14) together with the symmetry of $T$, there exists $\tilde{z} \in \tilde{Z}$ such that $f\left(x^{0}+\bar{z}\right) \in\{f(x+z)\}+\frac{1}{2} T \subseteq\{f(x+z)\}+T$, because $T$ is convex and $0 \in T$. Hence, we get from $(21)$

$$
f\left(x^{0}+\tilde{z}\right)-k \in(\{y\}+T) \cap\left(\operatorname{Sup}\left(f_{Z}\left(x^{0}\right)\right)-K\right) .
$$

By Assumption (19), $f\left(x^{0}+\tilde{z}\right)-k \notin \operatorname{Sup}\left(f_{Z}\left(x^{0}\right)\right)$.

We assume that (22) does not hold. Then, there exists $t \in T$ such that $y+t \notin$ $\operatorname{Sup}\left(f_{Z}\left(x^{0}\right)\right)-K$. Because $\{y\}+T$ is convex and due to (23), there exists

$$
w \in \operatorname{conv}\left\{f\left(x^{0}+\tilde{z}\right)-k, y+t\right\} \subseteq\{y\}+T
$$

such that, using (4),

$$
w \in \operatorname{bd}\left(\operatorname{Sup}\left(f_{Z}\left(x^{0}\right)\right)-K\right) \cap(\{y\}+T)=\operatorname{Sup}\left(f_{Z}\left(x^{0}\right)\right) \cap(\{y\}+T)
$$

in contradiction to (19). Hence, (22) holds and by using (20) we obtain

$$
\operatorname{cl}(\{y\}+T) \subseteq \operatorname{cl}\left(\operatorname{Sup}\left(f_{Z}\left(x^{0}\right)\right)-K\right)=\operatorname{cl}\left(f_{Z}\left(x^{0}\right)-K\right) .
$$

By Property (e) in Lemma 16 and Remark 1 we get

$$
y+a \in \operatorname{cl}(\{y\}+T) \subseteq \operatorname{cl}\left(f_{Z}\left(x^{0}\right)-K\right)=f_{Z}\left(x^{0}\right)-K .
$$

Hence, there exists $z^{\prime} \in Z$ such that $y+a \in\left\{f\left(x^{0}+z^{\prime}\right)\right\}-K$. Then, due to (13), it holds

$$
\{y\}+T \subseteq\{y+a\}-\operatorname{int}(K) \subseteq\left\{f\left(x^{0}+z^{\prime}\right)\right\}-\operatorname{int}(K) .
$$


According to Property (d) in Lemma 16 this is equivalent to

$$
\left\{f\left(x^{0}+z^{\prime}\right)\right\}+T \subseteq\{y\}+\operatorname{int}(K) .
$$

Due to (17), there exists $\tilde{z} \in Z$ such that $f(x+\tilde{z}) \in\left\{f\left(x^{0}+z^{\prime}\right)\right\}+T$. As a consequence, we finally obtain

$$
f(x+\tilde{z}) \in\left\{f\left(x^{0}+z^{\prime}\right)\right\}+T \subseteq\{y\}+\operatorname{int}(K)
$$

in contradiction to $y \in \operatorname{Sup}\left(f_{Z}(x)\right)$. Thus, (18) does not hold and we have

$$
\operatorname{Sup}\left(f_{Z}(x)\right) \subseteq \operatorname{Sup}\left(f_{Z}\left(x^{0}\right)\right)+T \subseteq \operatorname{Sup}\left(f_{Z}\left(x^{0}\right)\right)+B(0, \varepsilon) .
$$

\section{Relationship to Robustness Concepts in the Liter- ature}

This section compares decision robust efficiency to two types of robustness concepts in the literature. First, a minmax robust counterpart for decision uncertainty in single objective optimization is investigated. We show that decision robust efficiency can be considered as a general approach to decision uncertainty because it reduces to a scalar-valued decision robustness concept when applied to single objective problems. Secondly, we investigate a robustness concept for parameter uncertainty in multiobjective optimization. We show that decision robust efficiency can be considered a special case of the presented concept if the corresponding multiobjective problem with decision uncertainty is reformulated accordingly.

\subsection{Relationship to Minmax Robustness for Decision Uncertainty in Single-Objective Optimization}

Single-objective optimization with decision uncertainty has been studied for years in the areas robust optimization and robust optimal control, see [BS07] for a survey.

In single-objective robust optimization, the function $f: \mathbb{R}^{n} \rightarrow \mathbb{R}$ is replaced by the map

$$
x \mapsto \bar{f}(x):=\sup _{z \in Z} f(x+z),
$$

leading to the single-objective minmax robust counterpart

$$
\left(\mathrm{RC}^{s o}\right) \quad \min _{x \in X} \bar{f}(x) .
$$

The map $\bar{f}$ is studied in more detail under the name robust regularization in [Lew02] and [LP09]. For special cases of perturbation sets, the problem $\left(\mathrm{RC}^{s o}\right)$ has been studied under the name "robust optimization with implementation error", see, e.g., [BTH11, BNT07, BNT10a, SdH08]. 
The definition of the robust regularization $\bar{f}$ is given under the assumption that for all $x \in X$ the supremum satisfies $\sup _{z \in Z} f(x+z)<\infty$ and hence $\bar{f}: X \rightarrow \mathbb{R}$.

In the literature on single-objective optimization with decision uncertainty, $Z$ is widely defined as a convex compact neighborhood of 0 . However, we only require $0 \in Z$ and $Z$ compact. Proposition 19 shows that $f_{\text {Sup }}$, which is studied in Section 2.3, reduces to $\bar{f}$ in single-objective problems. Hence, the set of decision robust efficient solutions corresponds exactly to the minimizers of $\left(\mathrm{RC}^{s o}\right)$ if $k=1$ and $K=\mathbb{R}_{+}$. Our definition of decision robust efficiency can hence be considered as a generalization of the single-objective framework.

Proposition 19. Let $f: \Omega \rightarrow \mathbb{R}$ be continuous and let $K=\mathbb{R}_{+}$. Then we have for all $x^{*} \in X$

(i) $f_{\text {Sup }}\left(x^{*}\right)=\left\{\bar{f}\left(x^{*}\right)\right\}=\left\{\sup _{z \in Z} f\left(x^{*}+z\right)\right\}$,

(ii) $x^{*}$ is decision robust [weakly/./strictly] efficient in the sense of Definition 3 if and only if $x^{*}$ is [an/an/the unique] optimal solution of $\left(\mathrm{RC}^{s o}\right)$.

Proof. We consider dimension $k=1$, hence int $\left(\mathbb{R}_{+}\right)=\mathbb{R}_{+} \backslash\{0\}$ and decision robust efficiency coincides with decision robust weak efficiency. The $\operatorname{maximum}_{\max _{z \in Z}} f\left(x^{*}+z\right)=$ $\bar{f}\left(x^{*}\right)$ exists because $f$ is continuous and $Z$ is compact. Consequently, for all $x^{*} \in X$ it holds according to Definition 6

$$
\begin{aligned}
f_{\text {Sup }}(x) & =\left\{y \in \operatorname{cl}\left(f_{Z}(x)-\mathbb{R}_{+}\right) \mid \forall y^{\prime}>y: y^{\prime} \notin \operatorname{cl}\left(f_{Z}(x)-\mathbb{R}_{+}\right)\right\} \\
& =\left\{\sup _{z \in Z} f(x+z)\right\}=\left\{\max _{z \in Z} f(x+z)\right\} .
\end{aligned}
$$

Applying Theorem 9, we therefore obtain that $x^{*}$ is decision robust [weakly $/ \cdot /$ strictly] efficient if and only if $\max _{z \in Z} f(x+z)[\geq / \geq />] \max _{z \in Z} f\left(x^{*}+z\right)$ for all $x \in X \backslash\left\{x^{*}\right\}$, i.e., if and only if $x^{*}$ is [an/an/the unique] optimal solution of $\left(\mathrm{RC}^{s o}\right)$.

Remark 2. Result (i) in Proposition 19 also holds if $f$ is not continuous, but (ii) is less strong in that case. If $f$ is not continuous, it is straightforward to prove for all $x^{*} \in X$ :

- If $x^{*}$ is decision robust [weakly/·] efficient, then $x^{*}$ is an optimal solution of $\left(\mathrm{RC}^{s o}\right)$.

- If $x^{*}$ is the unique optimal solution of $\left(\mathrm{RC}^{s o}\right)$, then $x^{*}$ is decision robust strictly efficient.

\subsection{Relationship to Parameter Uncertainty in Multiobjective Op- timization}

Decision robust efficiency is a concept to handle decision uncertainty in multiobjective optimization. Parameter uncertainty in multiobjective optimization has recently been addressed with minmax robustness, see, e.g., [EIS14]. From a mathematical point of view, both concepts are related, as Proposition 21 shows. We first give the definition of robust efficiency for parameter uncertainty in multiobjective optimization problems. 
Definition 20 ([EIS14],[IKK $\left.\left.{ }^{+} 14\right]\right)$. Let $(\mathcal{P}(\xi), \xi \in \mathcal{U})$ be a family of optimization problems of the type

$$
(\mathcal{P}(\xi)) \min _{x \in \mathbb{R}^{n}}\left\{g(x, \xi) \mid G_{j}(x, \xi) \leq 0, \forall 1 \leq j \leq m\right\},
$$

where $\mathcal{U} \subseteq \mathbb{R}^{p}, g: \mathbb{R}^{n} \times \mathcal{U} \rightarrow \mathbb{R}^{k}$ and $G: \mathbb{R}^{n} \times \mathcal{U} \rightarrow \mathbb{R}^{m}$. A point $x \in \mathbb{R}^{n}$ is called robust feasible for $(\mathcal{P}(\xi), \xi \in \mathcal{U})$ if $x$ is feasible for $(\mathcal{P}(\xi))$ for all $\xi \in \mathcal{U}$.

A robust feasible solution $x \in \mathbb{R}^{n}$ is called robust [weakly/./strictly] efficient for $(\mathcal{P}(\xi), \xi \in$ $\mathcal{U})$ if there is no other robust feasible $y \neq x$ such that

$$
g_{\mathcal{U}}(y) \subseteq g_{\mathcal{U}}(x)-[\operatorname{int}(K) / K \backslash\{0\} / K],
$$

where $g_{\mathcal{U}}(y)=\{g(y, \xi) \mid \xi \in \mathcal{U}\}$ for $y \in \mathbb{R}^{n}$. In case of $K=\mathbb{R}_{+}^{k}$, each robust [weakly/·/strictly] efficient solution is called a minmax robust [weakly/./strictly] efficient solution.

In the following we show that we can rewrite the multiobjective problem with decision uncertainty $(\mathcal{P}(z), z \in Z)$ to an equivalent problem with parameter uncertainty and that the decision robust [weakly/./strictly] efficient solutions for the former coincide with the robust [weakly $/ \cdot /$ strictly] efficient solutions for the latter.

Proposition 21. (i) Every family $(\mathcal{P}(z), z \in Z)$, where

$$
\mathcal{P}(z)=\min _{x \in \mathbb{R}^{n}}\left\{f(x+z) \mid F_{j}(x+z) \leq 0 \forall 1 \leq j \leq m\right\}
$$

can be written as a family $(\mathcal{P}(\xi), \xi \in \mathcal{U})$ of uncertain multiobjective optimization problems in the sense of Definition 20, where $\mathcal{U}=Z, g(x, \xi)=f(x+\xi)$ and $G_{j}(x, \xi)=$ $F_{j}(x+\xi)$ for $x \in \Omega, \xi \in \mathcal{U}$.

(ii) A solution $x \in \Omega$ is decision robust [weakly/./strictly] efficient for ( $\mathrm{RC}$ ) if and only if it is robust [weakly/./strictly] efficient w.r.t. the family of uncertain multiobjective optimization problems according to (i).

Proof. (i) Plugging the definitions of $\mathcal{U}, g: \Omega \times \mathcal{U} \rightarrow \mathbb{R}^{k}$, and $G: \Omega \times \mathcal{U} \rightarrow \mathbb{R}^{m}$ into $(\mathcal{P}(\xi))$ results exactly in $(\mathcal{P}(z))$ for all $z \in Z$.

(ii) A solution $x$ is robust feasible in the sense of Definition 20 if and only if $G_{j}(x, \xi) \leq 0$ for all $1 \leq j \leq m$ and $\xi \in \mathcal{U}$. Then

$$
\begin{array}{lr}
\quad x \text { is decision robust feasible } \\
\Leftrightarrow & \forall z \in \mathcal{U}=Z \forall 1 \leq j \leq m: 0 \geq F_{j}(x+z)=G_{j}(x, z) \\
\Leftrightarrow & x \text { is robust feasible in the sense of Definition } 20 .
\end{array}
$$

From the definitions of $g$ and $\mathcal{U}$ in (i) we have $f_{Z}(x)=g_{\mathcal{U}}(x)$ for all $x \in X=\left\{y \in \mathbb{R}^{n} \mid\right.$ $\left.F_{j}(y+z) \leq 0 \forall z \in Z \forall 1 \leq j \leq m\right\}=\left\{y \in \mathbb{R}^{n} \mid G_{j}(y, z) \leq 0 \forall z \in \mathcal{U} \forall 1 \leq j \leq m\right\}$. 
Hence,

$$
\begin{array}{ll} 
& x \in X \text { is decision robust [weakly/·/strictly] efficient for (RC) } \\
\Leftrightarrow & \forall y \in X \backslash\{x\}: f_{Z}(y) \nsubseteq f_{Z}(x)-[\operatorname{int}(K) / K \backslash\{0\} / K] \\
\Leftrightarrow & \forall y \in X \backslash\{x\}: g_{\mathcal{U}}(y) \nsubseteq g_{\mathcal{U}}(x)-[\operatorname{int}(K) / K \backslash\{0\} / K] \\
\Leftrightarrow & x \in X \text { is robust [weakly///strictly] efficient w.r.t. Definition } 20 .
\end{array}
$$

Hence, from the mathematical point of view, decision uncertainty can be considered as a special case of parameter uncertainty and the results of [EIS14] apply. However, the setting of decision uncertainty in multiobjective optimization adds a considerable amount of structure to the robust counterpart leading to significant results that do not hold for parameter uncertainty. In particular, a research gap stated in [EIS14, p. 30] is that there exists no method to calculate all robust efficient solutions for parameter uncertainty in multiobjective optimization, not even for linear objectives. However, for decision uncertainty in multiobjective optimization with linear objective functions all decision robust efficient solutions can be determined by methods from deterministic multiobjective optimization, which is shown in Section 5. Further results are gained for two other specific classes of objective functions: Lipschitz continuous or monotonic objective functions. To the best of our knowledge, no such results exist for parameter uncertainty. Moreover, in Section 3.2, the specific structure of decision uncertainty leads to the presented continuity properties of the set-valued functions $f_{Z}$ and $f_{\text {Sup }}$.

\section{$5 \quad$ Results for Special Classes of Objective Functions}

In the following, we investigate properties of the decision robust counterpart (RC) for three distinct types of objective functions. The types are linear, Lipschitz continuous and monotonic objective functions. For linear objective functions, we show that the decision robust [weakly $/ \cdot /$ strictly] efficient solutions can be determined by methods from deterministic linear multiobjective optimization. A necessary condition for decision robust efficient solutions is given in case of Lipschitz continuous objective functions. We also present a sufficient condition for decision robust efficient solutions in case of monotonic objective functions.

\subsection{Linear Objective Functions}

In this section we look at the case where the objective function $f$ is linear. We prove that each decision robust feasible solution is decision robust [weakly $/ \cdot /$ strictly] efficient for $(\mathcal{P}(z), z \in Z)$ if and only if it is [weakly/./strictly] efficient for the deterministic multiobjective problem

$$
\left(\left.\mathcal{P}\right|_{X}\right) \quad \min _{x \in X} f(x) .
$$

As a preparation for the main result of this section, we state the following lemma. 
Lemma 22. Let $K$ be a closed convex pointed solid cone in $\mathbb{R}^{k}$. Let $v \in \mathbb{R}^{k}$ such that $v \notin[\operatorname{int}(K) / K \backslash\{0\} / K]$. Then there exists $c \in \mathbb{R}^{k}$ such that $\langle c, v\rangle\langle\langle c, k\rangle$ for all $k \in$ $[\operatorname{int}(K) / K \backslash\{0\} / K]$.

Proof. In case of $v \notin K$ or $v \notin$ int $(K)$ the claim corresponds to the Theorem of HahnBanach, see, e.g., [Rud91].

For the case $v \notin K \backslash\{0\}$ we distinguish two cases: If $v \neq 0$ we can separate $v$ from $K$, hence also from $K \backslash\{0\}$. Now, let $v=0$. Because $K$ is closed, convex and pointed, we have

$$
K^{\#}=\left\{d \in \mathbb{R}^{k} \mid\langle d, k\rangle>0, \forall k \in K \backslash\{0\}\right\} \neq \emptyset
$$

due to Theorem 3.38 in [Jah11]. Hence, for each $c \in K^{\#}$ and all $k \in K \backslash\{0\}$ it holds $\langle c, k\rangle>0=\langle c, v\rangle$.

Now, we can prove our main result about decision robust [weakly/./strictly] efficient solutions to uncertain problems with linear objective functions.

Theorem 23. Let $f: \Omega \rightarrow \mathbb{R}^{k}$ be linear. Then for each decision robust feasible solution $x \in X$

$$
\begin{array}{r}
x \text { is [weakly/./strictly] efficient for }\left(\left.\mathcal{P}\right|_{X}\right) \\
\Leftrightarrow \quad x \text { is decision robust [weakly/./strictly] efficient for }(\mathcal{P}(z), z \in Z) .
\end{array}
$$

According to this theorem, solving the deterministic linear multiobjective problem $\left(\left.\mathcal{P}\right|_{X}\right)$, is equivalent to determining decision robust efficient solutions. Hence, linear multiobjective problems including decision uncertainty can be solved by any solution method for deterministic linear multiobjective optimization. This is particularly interesting as a method to compute all robust efficient solutions for linear multiobjective problems with parameter uncertainty is not known so far, see [EIS14].

Proof. We begin by showing that every [weakly $/ \cdot /$ strictly] efficient solution for $\left(\left.\mathcal{P}\right|_{X}\right)$ is also decision robust [weakly/./strictly] efficient for $(\mathcal{P}(z), z \in Z)$. Let $x \in X$ be [weakly $/ \cdot /$ strictly] efficient for $\left(\left.\mathcal{P}\right|_{X}\right)$ and assume that $x$ is not decision robust [weakly $/ \cdot /$ strictly] efficient for $(\mathcal{P}(z), z \in Z)$. Consequently, there exists $y \in X \backslash\{x\}$ such that $f_{Z}(y) \subseteq$ $f_{Z}(x)-[\operatorname{int}(K) / K \backslash\{0\} / K]$.

Since $x$ is $[$ weakly $/ \cdot /$ strictly] efficient

$$
v:=f(x)-f(y)=f(x-y) \notin[\operatorname{int}(K) / K \backslash\{0\} / K] .
$$

According to Lemma 22 there exists $c \in \mathbb{R}^{k}$ such that

$$
\langle c, v\rangle<\langle c, k\rangle \quad \forall k \in[\operatorname{int}(K) / K \backslash\{0\} / K] .
$$

Choose $\tilde{z} \in \operatorname{argmax}_{z \in Z}\langle c, f(z)\rangle$, which is nonempty, because $f$ and the inner product are continuous and $Z$ is compact. Hence,

$$
\langle c, f(z-\tilde{z})\rangle \leq 0 \quad \forall z \in Z .
$$


Because $f_{Z}(y) \subseteq f_{Z}(x)-[\operatorname{int}(K) / K \backslash\{0\} / K]$, there exists $z \in Z$ and $k \in[\operatorname{int}(K) / K \backslash\{0\} / K]$ such that $f(y+\tilde{z})=f(x+z)-k$. Then, by using $(25)$,

$$
\begin{array}{ll} 
& k=f(x-y)+f(z-\tilde{z})=v+f(z-\tilde{z}) \\
\Rightarrow & \langle c, k\rangle=\langle c, v\rangle+\langle c, f(z-\tilde{z})\rangle \leq\langle c, v\rangle \\
\Rightarrow & \langle c, k\rangle \leq\langle c, v\rangle
\end{array}
$$

in contradiction to Equation (24). Therefore, $x$ is decision robust [weakly/./strictly] efficient.

Now, the opposite implication is proven. Assume that $x$ is not [weakly/./strictly] efficient for $\left(\left.\mathcal{P}\right|_{X}\right)$. We show that $x$ can not be decision robust [weakly $/ \cdot /$ strictly] efficient in this case.

$$
\begin{aligned}
& x \text { is not [weakly/·strictly] efficient } \\
& \Rightarrow \exists y \in X \backslash\{x\}: f(y) \in\{f(x)\}-[\operatorname{int}(K) / K \backslash\{0\} / K] \\
& \Rightarrow \quad \exists y \in X \backslash\{x\}: \forall z \in Z \quad f(y+z) \in\{f(x+z)\}-[\operatorname{int}(K) / K \backslash\{0\} / K] \\
& \Rightarrow \quad \exists y \in X \backslash\{x\}: \forall z \in Z \exists z^{\prime}=z \in Z: f(y+z) \in\left\{f\left(x+z^{\prime}\right)\right\}-[\operatorname{int}(K) / K \backslash\{0\} / K] \\
& \Rightarrow \quad x \text { is not decision robust [weakly/./strictly] efficient. }
\end{aligned}
$$

\subsection{Lipschitz Continuous Objective Functions}

In general, decision robust efficient solutions for $(\mathcal{P}(z), z \in Z)$ are not efficient for $\left(\left.\mathcal{P}\right|_{X}\right)$. As Example 1 shows, this is not even true for Lipschitz objective functions, since the objective function is Lipschitz on the compact feasible set $\Omega$. Nevertheless, in case of Lipschitz objective functions, every decision robust efficient solution is at least approximately efficient. In view of that, we prove a necessary condition for a decision robust efficient solution.

Recall that a function $f: \mathbb{R}^{n} \rightarrow \mathbb{R}^{k}$ is called Lipschitz continuous or Lipschitz with Lipschitz constant $L>0$ if for all $x, y \in \mathbb{R}^{n}$

$$
\|f(x)-f(y)\| \leq L \cdot\|x-y\| .
$$

Several concepts of approximate solutions for multiobjective optimization problems have been introduced in the literature see for instance [Dur07] and the references therein. We use the following concept which goes back to Kutateladze [Kut79].

Definition 24. Let $k^{0} \in K \backslash\{0\}$ and $\varepsilon>0$ be given. The point $x^{*} \in X$ is a [weakly/·strictly] $\left(\varepsilon, k^{0}\right)$-minimal solution for $\left(\left.\mathcal{P}\right|_{X}\right)$, i.e., $\min _{x \in X} f(x)$, if there exists no $x \in X$ such that

$$
f(x) \in\left\{f\left(x^{*}\right)-\varepsilon k^{0}\right\}-[\operatorname{int}(K) / K \backslash\{0\} / K] .
$$

In the following we assume w.l.o.g. $\left\|k^{0}\right\|=1$ in the definition above. 
Theorem 25. Let $f: \mathbb{R}^{n} \rightarrow \mathbb{R}^{k}$ be Lipschitz continuous with Lipschitz constant $L>0$ and $Z \neq\{0\}$. Furthermore, set

$$
\tilde{L}:=L \cdot \max _{z \in Z}\|z\| \quad \text { and } \quad S:=\bigcap_{h \in \bar{B}(0, \tilde{L})}\{h\}+K .
$$

If $x^{*} \in X$ is decision robust [weakly/·strictly] efficient for $(\mathcal{P}(z), z \in Z)$, then there exists no $\tilde{x} \in X$ and no $s \in S$ such that $f(\tilde{x}) \in\left\{f\left(x^{*}\right)-s\right\}-[\operatorname{int}(K) / K \backslash\{0\} / K]$. Hence, $x^{*}$ is an [weakly/·/strictly] $\left(\varepsilon, k^{0}\right)$-minimal solution for $\left(\left.\mathcal{P}\right|_{X}\right)$, i.e., $\min _{x \in X} f(x)$, where

$$
k^{0}=\frac{s}{\|s\|} \quad \text { and } \quad \varepsilon=\|s\|
$$

for all $s \in S$.

Proof. First we note that $S \subseteq K \backslash\{0\}$ : as $K$ is pointed and solid there exists $\tilde{h} \in \operatorname{int}(K) \cap$ $\bar{B}(0, \tilde{L})$ and thus we have $S \subseteq\{\tilde{h}\}+K \subseteq \operatorname{int}(K)$ and $0 \notin S$.

Because $f$ is Lipschitz continuous, we have for all $x \in X$ and the closed ball of radius $\tilde{L}, \bar{B}(0, \tilde{L})$,

$$
f_{Z}(x) \subseteq\{f(x)\}+\bar{B}(0, \tilde{L})
$$

Consequently, for each $x \in X$ and $z \in Z$ there exists $h \in \bar{B}(0, \tilde{L})$ such that $f(x+z)=$ $f(x)+h$. Furthermore, it holds $s \in S$ if and only if $h \in\{s\}-K$ for all $h \in \bar{B}(0, \tilde{L})$.

Let $x^{*} \in X$ be decision robust [weakly/./strictly] efficient and suppose there exists $\tilde{x} \in X$ and $s \in S$ such that

$$
f(\tilde{x}) \in\left\{f\left(x^{*}\right)-s\right\}-[\operatorname{int}(K) / K \backslash\{0\} / K] .
$$

Then, for each $z \in Z$ there exists $h \in \bar{B}(0, \tilde{L})$ such that

$$
\begin{aligned}
f(\tilde{x}+z) & =f(\tilde{x})+h \\
& \in\{f(\tilde{x})\}+(\{s\}-K) \\
& \subseteq\left\{f\left(x^{*}\right)-s\right\}+\{s\}-[\operatorname{int}(K) / K \backslash\{0\} / K] \\
& =\left\{f\left(x^{*}\right)\right\}-[\operatorname{int}(K) / K \backslash\{0\} / K] \\
& \subseteq f_{Z}\left(x^{*}\right)-[\operatorname{int}(K) / K \backslash\{0\} / K],
\end{aligned}
$$

in contradiction to $x^{*}$ being decision robust [weakly/./strictly] efficient.

\subsection{Monotonic Objective Functions}

In the following, we look at the special case of monotonically decreasing and increasing objective functions. We show that, in case of at least one increasing objective, one decreasing objective and a partially ordered feasible set, all solutions are decision robust efficient.

Throughout this section, we assume that $\mathbb{R}^{k}$ is partially ordered by the closed convex pointed solid cone $\mathbb{R}_{+}^{k}$ and that $\mathbb{R}^{n}$ is ordered correspondingly by the cone $\mathbb{R}_{+}^{n}$. First, we recall the definition of a monotonically increasing function on $\mathbb{R}^{n}$, see, e.g., [Jah11, Mie12]. 
Definition 26. Let $S$ be a nonempty subset of $Y \subseteq \mathbb{R}^{n}$. Then the function $h: Y \rightarrow \mathbb{R}$ is called

(a) monotonically increasing on $S$ if for all $x, y \in S$ it holds

$x \leqq y \Rightarrow h(x) \leq h(y)$.

(b) strictly monotonically increasing on $S$ if for all $x, y \in S$ it holds

$x<y \Rightarrow h(x)<h(y)$.

(c) strongly monotonically increasing on $S$ if for all $x, y \in S$ it holds

$x \leq y \Rightarrow h(x)<h(y)$.

Similarly, $f$ is called decreasing if $(-f)$ is increasing.

Note that every strongly increasing function is also strictly increasing and increasing, while strictly increasing functions are not necessarily increasing if $n \geq 2$. For $n=1$, strict and strong monotonicity coincide and every strictly increasing function is also increasing.

Theorem 27. Let $X \subseteq \Omega \subseteq \mathbb{R}^{n}$ and let $x \in X$ be such that for all $y \in X$

$$
x \neq y \quad \Rightarrow \quad x[\leq /</ \leq] y \text { or } y[\leq /</ \leq] x
$$

and let $Z \subseteq \mathbb{R}^{n}$ have the property

$$
z^{1} \neq z^{2} \quad \Rightarrow \quad z^{1}[\leq /</ \leq] z^{2} \quad \text { or } \quad z^{2}[\leq /</ \leq] z^{1}
$$

for all $z^{1}, z^{2} \in Z$.

Let $k \geq 2$ and let $f: \mathbb{R}^{n} \rightarrow \mathbb{R}^{k}$ have the property that there exist $j, l \in\{1, \ldots, k\}, j \neq l$, such that

(a) $f_{j}: \mathbb{R}^{n} \rightarrow \mathbb{R}$ is $[. /$ strictly/strongly $]$ increasing on $X$

(b) $f_{l}: \mathbb{R}^{n} \rightarrow \mathbb{R}$ is $[. /$ strictly/strongly $]$ decreasing on $X$.

Then $x$ is decision robust [weakly/strictly/strictly]-efficient.

Proof. Choose $y \in X \backslash\{x\}$ arbitrarily. Because $Z$ is compact and totally ordered with respect to $\leqq$, there exist a minimal element $z^{\min } \in Z$ and a maximal element $z^{\max } \in Z$ due to Zorn's Lemma.

We distinguish two cases. First, we study the case $x[\leq /</ \leq]$. This leads to

$$
\begin{array}{lll}
\stackrel{(a)}{\Rightarrow} & f_{j}(x+z) \quad[\leq /</<] \quad f_{j}\left(y+z^{\text {max }}\right) \quad \forall z \in Z \\
\Rightarrow & f\left(y+z^{\text {max }}\right) \notin f_{Z}(x)-\left[\operatorname{int}\left(\mathbb{R}_{+}^{k}\right) / \mathbb{R}_{+}^{k} / \mathbb{R}_{+}^{k}\right] \\
\Rightarrow & f_{Z}(y) \nsubseteq f_{Z}(x)-\left[\operatorname{int}\left(\mathbb{R}_{+}^{k}\right) / \mathbb{R}_{+}^{k} / \mathbb{R}_{+}^{k}\right] .
\end{array}
$$


Secondly, we study the alternative case $y[\leq /</ \leq] x$. This leads to

$$
\begin{array}{lll} 
& y+z^{\text {min }} \quad[\leq /</ \leq] \quad x+z^{\text {min }} \leqq x+z \quad \forall z \in Z \\
\stackrel{(b)}{\Rightarrow} & f_{l}\left(y+z^{m i n}\right) \quad[\geq />/>] \quad f_{l}(x+z) \quad \forall z \in Z \\
\Rightarrow & f\left(y+z^{m i n}\right) \notin f_{Z}(x)-\left[\operatorname{int}\left(\mathbb{R}_{+}^{k}\right) / \mathbb{R}_{+}^{k} / \mathbb{R}_{+}^{k}\right] \\
\Rightarrow & f_{Z}(y) \nsubseteq f_{Z}(x)-\left[\operatorname{int}\left(\mathbb{R}_{+}^{k}\right) / \mathbb{R}_{+}^{k} / \mathbb{R}_{+}^{k}\right] .
\end{array}
$$

In summary, there is no $y \in X \backslash\{x\}$ such that $f_{Z}(y) \subseteq f_{Z}(x)-\left[\operatorname{int}\left(\mathbb{R}_{+}^{k}\right) / \mathbb{R}_{+}^{k} / \mathbb{R}_{+}^{k}\right]$. Consequently, $x$ is decision robust [weakly/strictly/strictly]-efficient.

For an illustarion of Theorem 27, we refer to Example 4 in Appendix A.

A direct consequence of Theorem 27 is the next Corollary.

Corollary 28. Let $Z \subseteq \mathbb{R}$ and $X \subseteq \Omega \subseteq \mathbb{R}$ be compact intervals and $f: \mathbb{R} \rightarrow \mathbb{R}^{k}$ be such that $f_{1}: \mathbb{R} \rightarrow \mathbb{R}$ is [./strictly increasing and $f_{2}: \mathbb{R} \rightarrow \mathbb{R}$ is [./strictly] decreasing.

Then all $x \in X$ are decision robust [weakly/strictly]-efficient.

In particular, Corollary 28 can be applied whenever $f: I \rightarrow \mathbb{R}^{k}$ is a curve with at least one increasing and one decreasing objective, where $I \subseteq \mathbb{R}$ is an interval.

\section{Conclusions}

A robustness concept for decision uncertainty in multiobjective optimization is introduced and investigated. The corresponding decision robust counterpart (RC) is introduced as a set-valued optimization problem. Semicontinuity and convexity of the set-valued objective functions in (RC) and their supremal sets are proven under the assumption that the vectorvalued objective function in $(\mathcal{P})$ is continuous.

Results are obtained that lead to the determination of decision robust efficient solutions by methods from deterministic multiobjective optimization for three types of objective functions. First, in case of linear objective functions, solving (RC) is equivalent to solving a deterministic linear multiobjective problem. Second, a necessary condition for decision robust efficient solutions in case of Lipschitz objective functions is presented. Third, a sufficient condition for decision robust efficient solutions in case of monotonic objective functions is obtained.

Decision robust efficiency and (RC) are consistent with the generalization of the supremum to supremal sets in set-valued optimization. Furthermore, decision robust efficiency is closely related to existing robustness concepts in the literature in two ways. On the one hand, it is a generalization of minmax robustness approaches for decision uncertainty in single-objective optimization and on the other hand, it can be regarded as a special case of minmax robust efficiency for parameter uncertainty in multiobjective optimization.

Following the presented theoretical results, there are different options for further research. The results for the different classes of objective functions indicate that extended study of problems with specific structure might lead to specific solution techniques for 
various applications. In particlular, solution techniques and numerical evaluations of the growing media mixing problem presented in the introduction are currently under research, see [CKGS17]. Therein, the investigation of monotonic functions, as studied in Theorem 27 , is extended to piecewise monotonic functions with two objectives and the results are then used to calculate decision robust efficient solutions for a case study on an Italian plant nursery.

Additionally, the problem description of decision uncertainty can be extended by allowing the perturbation set $Z$ to depend on the solution. There are various applications for problems with variable perturbation sets, including perturbation sets whose size depend on a percentage of the solution aimed at. A specific case of variable perturbation sets that would be interesting to study is the relative implementation error, which is also studied in single objective robust optimization see, e.g., [SdH08, BTN02]. This error affects each component of a solution $x$ by multiplication, i.e., each component $x_{i}$ is realized within the set $\left\{\left(1+\xi_{i}\right) x_{i}|| \xi_{i} \mid<\varepsilon_{i}\right\}$, leading to the perturbation set $Z(x)=\left[-\varepsilon_{1} x_{1}, \varepsilon_{1} x_{1}\right] \times \cdots \times\left[-\varepsilon_{n} x_{n}, \varepsilon_{n} x_{n}\right]$. We note that the solution 0 is not affected by the relative error. However, decision uncertainty in the solution 0 is required by the applications presented in Section 1. In addition, for perturbation sets that vary with different solutions, we expect that objective function dependent solution approaches like in Section 5 will be harder to find and be more expensive to use than the results of this work.

\section{Acknowledgements}

The authors thank Professor Margaret Wiecek for her valuable comments on the paper. Furthermore, the authors thank two anonymous referees for their helpful and detailed remarks.

\section{References}

[AB08] Gideon Avigad and Jürgen Branke. Embedded evolutionary multi-objective optimization for worst case robustness. In Proceedings of the 10th Annual Conference on Genetic and Evolutionary Computation, GECCO '08, pages 617-624, New York, NY, USA, 2008. ACM.

[AF90] J.P. Aubin and H. Frankowska. Set-valued analysis. Birkhäuser, Boston, Basel, Berlin, 1990.

[BA06] C. Barrico and C.H. Antunes. Robustness analysis in multi-objective optimization using a degree of robustness concept. In IEEE Congress on Evolutionary Computation. CEC 2006., pages 1887 -1892. IEEE Computer Society, 2006.

[BN10] D. Bertsimas and O. Nohadani. Robust optimization with simulated annealing. Journal of Global Optimization, 48(2):323-334, 2010. 
[BNT07] D. Bertsimas, O. Nohadani, and K. M. Teo. Robust optimization in electromagnetic scattering problems. Journal of Applied Physics, 101(7):074507, 2007.

[BNT10a] D. Bertsimas, O. Nohadani, and K. M. Teo. Nonconvex robust optimization for problems with constraints. INFORMS Journal on Computing, 22(1):44-58, 2010 .

[BNT10b] D. Bertsimas, O. Nohadani, and K. M. Teo. Robust optimization for unconstrained simulation-based problems. Operations Research, 58(1):161-178, 2010 .

[BP03] J. Benoist and N. Popovici. Characterizations of convex and quasiconvex setvalued maps. Math. Meth. of OR, 57(3):427-435, 2003.

[BS07] H. G. Bayer and B. Sendhoff. Robust Optimization - A Comprehensive Survey. Computer Methods in Applied Mechanics and Engineering, 196(33-34):3190$3218,2007$.

[BTGN09] A. Ben-Tal, L. El Ghaoui, and A. Nemirovski. Robust Optimization. Princeton University Press, Princeton and Oxford, 2009.

[BTH11] A. Ben-Tal and D. Den Hertog. Immunizing conic quadratic optimization problems against implementation errors. CentER Working Paper Series, 2011060, 2011.

[BTN02] A. Ben-Tal and A. Nemirovski. Robust optimization - methodology and applications. Mathematical Programming, 92(3):453-480, 2002.

[CKGS17] F. Castellani, C. Krüger, J. Geldermann, and A. Schöbel. Peat and pots: resource efficiency by decision robust efficiency. Working paper, 2017.

[Das97] Indraneel Das. Nonlinear multicriteria optimization and robust optimality. PhD thesis, Rice University, 1997.

[DD79] J.P. Delahaye and J. Denel. The continuities of the point-to-set maps, definitions and equivalences. Mathematical Programming Study, 10:8-12, 1979.

[DG06] K. Deb and H. Gupta. Introducing robustness in multi-objective optimization. Evolutionary Computation, 14(4):463-494, 2006.

[DTE14] M. Porcelli D. Terzijska and G. Eichfelder. Multi-objective optimization in the Lorentz force velocimetry framework. In Book of digests $\&$ program / OIPE, International Workshop on Optimization and Inverse Problems in Electromagnetism, volume 13, pages 81-82. Delft, 2014. 
[Dur07] M. Durea. On the existence and stability of approximate solutions of perturbed vector equilibrium problems. Journal of Mathematical Analysis and Applications, 333(2):1165-1179, 2007.

[EIS14] M. Ehrgott, J. Ide, and A. Schöbel. Minmax robustness for multi-objective optimization problems. European Journal of Operational Research, 239:17-31, 2014.

[EJ12] G. Eichfelder and J. Jahn. Vector optimization problems and their solution concepts. In Q. H. Ansari and J. C. Yao, editors, Recent Developments in Vector Optimization, pages 1-27. Springer, Berlin Heidelberg, 2012.

[FW14] J. Fliege and R. Werner. Robust multiobjective optimization \& applications in portfolio optimization. European Journal of Operational Research, 234(2):422 $-433,2014$.

[GJLVP14] M. A. Goberna, V. Jeyakumar, G. Li, and J. Vicente-Pérez. Robust solutions of multiobjective linear semi-infinite programs under constraint data uncertainty. SIAM Journal on Optimization, 24(3):1402-1419, 2014.

[GLP13] P.G. Georgiev, D.T. Luc, and P.M. Pardalos. Robust aspects of solutions in deterministic multiple objective linear programming. European Journal of Operational Research, 229(1):29 - 36, 2013.

[HJ11] T.X.D. Ha and J. Jahn. New order relations in set optimization. J. Optim. Theory Appl., 148:209-236, 2011.

$\left[\mathrm{IKK}^{+} 14\right] \quad J$. Ide, E. Köbis, D. Kuroiwa, A. Schöbel, and C. Tammer. The relationship between multi-objective robustness concepts and set valued optimization. Fixed Point Theory and Applications, 2014(83), 2014.

[IS16] J. Ide and A. Schöbel. Robustness for uncertain multi-objective optimization: A survey and analysis of different concepts. OR Spectrum, 38(1):235-271, 2016.

[Jah11] J. Jahn. Vector Optimization (2. ed.). Springer, Berlin, Heidelberg, 2011.

[Jah15] J. Jahn. A derivative-free descent method in set optimization. Computational Optimization and Applications, 60(2):393-411, 2015.

[KL12] D. Kuroiwa and G. M. Lee. On robust multiobjective optimization. Vietnam Journal of Mathematics, 40(2\&3):305-317, 2012.

[KTZ15] A.A. Khan, C. Tammer, and C. Zălinescu. Set-valued Optimization - an Introduction with Applications. Springer, Berlin Heidelberg, 2015.

[Kur96] D. Kuroiwa. Convexity for set-valued maps. Appl. Math. Lett., 9(2):97-101, 1996. 
[Kur98] D. Kuroiwa. The natural criteria in set-valued optimization. RIMS Kokyuroku, 1031:85-90, 1998.

[Kut79] S.S. Kutateladze. Convex E-programming. Soviet Math Dokl, 20(2):391-393, 1979.

[Lew02] A.S. Lewis. Robust regularization. Technical report, School of ORIE, Cornell University, Ithaca, NY, 2002. Available online at http://people.orie. cornell .edu/aslewis/publications/2002.html.

[Löh11] A. Löhne. Vector Optimization with Infimum and Supremum. Vector Optimization. Springer, Heidelberg, Berlin, 2011.

[LP09] A.S. Lewis and C.H.J. Pang. Lipschitz behavior of the robust regularization. SIAM Journal on Control and Optimization, 48(5):3080-3105, 2009.

[Mie12] K. Miettinen. Nonlinear multiobjective optimization, volume 12. Springer, 2012 .

[Nie80] J.W. Nieuwenhuis. Supremal points and generalized duality. Optimization, 11(1):41-59, 1980.

[RMS07] L. Rodríguez-Marín and M. Sama. $(\Lambda, C)$-contingent derivatives of set-valued maps. J. Math. Anal. Appl., 335:974-989, 2007.

[Rud91] W. Rudin. Functional analysis. McGraw-Hill Inc., New York, second edition, 1991.

[SdH08] E. Stinstra and D. den Hertog. Robust optimization using computer experiments. European Journal of Operational Research, 191(3):816 - 837, 2008.

[WD16] Margaret M. Wiecek and Garrett M. Dranichak. Robust Multiobjective Optimization for Decision Making Under Uncertainty and Conflict, chapter 4, pages 84-114. INFORMS, 2016.

\section{Appendix A An Example for Monotonic Objective Functions}

This example illustrates Theorem 27 and shows that, after having excluded a specific part of the decision robust feasible set, all decision robust efficient solutions can be determined by Theorem 27 .

Example 4. Let the feasible set, the perturbation set and the objective function be

$$
\begin{array}{ll} 
& \Omega=\operatorname{conv}\left\{\left(\begin{array}{l}
0 \\
0
\end{array}\right),\left(\begin{array}{c}
1.5 \\
0
\end{array}\right),\left(\begin{array}{c}
1.5 \\
1
\end{array}\right),\left(\begin{array}{c}
1 \\
1
\end{array}\right)\right\}, \quad Z=[0,0.5] \times\{0\} \\
\text { and } & f: \mathbb{R}^{2} \rightarrow \mathbb{R}^{2}, \quad x \mapsto\left(\begin{array}{c}
-\left(x_{1}+x_{2}\right) \\
x_{1}^{2}+x_{2}^{2}
\end{array}\right) .
\end{array}
$$


Then $f_{1}$ is strongly decreasing and $f_{2}$ is strongly increasing on $\mathbb{R}_{+}^{2}$ and therefore also on the decision robust feasible subset $X$ of $\Omega$, which is illustrated in Figure 4.
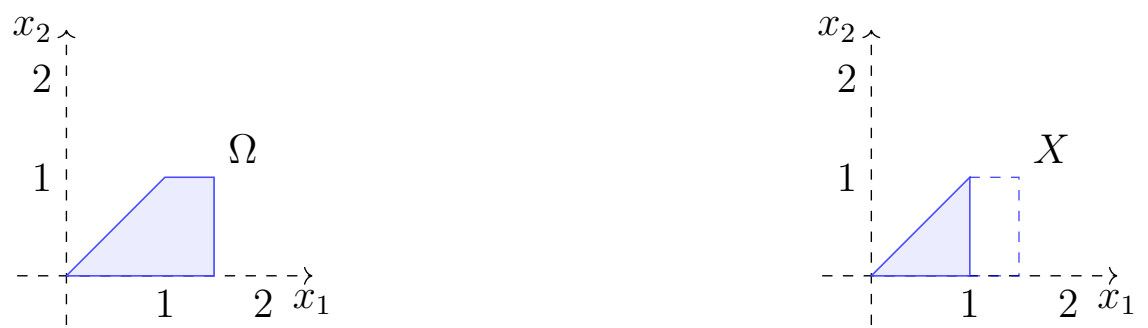

Figure 4: The feasible set $\Omega$ and the decision robust feasible set $X$ in Example 4 .

We show next that the set of decision robust [//strictly] efficient solutions is

$$
Y=\operatorname{conv}\left\{\left(\begin{array}{l}
0 \\
0
\end{array}\right),\left(\begin{array}{l}
1 \\
1
\end{array}\right)\right\} .
$$

We first show that the elements of $X \backslash Y$ can not be decision robust efficient.

For all $s \in(0,2)$ we define the set $L_{s}=\left\{x \in X \mid x_{1}+x_{2}=s\right\}$ and $y^{s}=\frac{1}{2} \cdot\left(\begin{array}{l}s \\ s\end{array}\right) \in L_{s}$, which is illustrated in Figure 5.

Let $s \in(0,2)$ and let $x \in L_{s} \backslash\left\{y^{s}\right\}$. Then $x_{1}>x_{2}$ and there exists $0<t \leq \frac{s}{2}$ such that $x_{1}=\frac{s}{2}+t$ and $x_{2}=\frac{s}{2}-t$. For each $z \in Z$ it holds

$$
f_{1}(x+z)=-\left(x_{1}+x_{2}+z_{1}\right)=-\left(s+z_{1}\right)=-\left(y_{1}^{s}+y_{2}^{s}+z_{1}\right)=f_{1}\left(y^{s}+z\right)
$$

as well as

$$
f_{2}\left(y^{s}+z\right)=\frac{s^{2}}{2}+s \cdot z_{1}+z_{1}^{2}
$$

and

$$
f_{2}(x+z)=\underbrace{\frac{s^{2}}{2}+s \cdot z_{1}+z_{1}^{2}}_{=f_{2}\left(y^{s}+z\right)}+\underbrace{2 t^{2}}_{>0}+\underbrace{2 t z_{1}}_{\geq 0}>f_{2}\left(y^{s}+z\right) .
$$

Consequently, $f\left(y^{s}+z\right) \leq f(x+z)$ for all $z \in Z$. Since $x \in L_{s}$ was arbitrarily chosen, we have for all $x \in L_{s} \backslash\left\{y^{s}\right\}$

$$
f_{Z}\left(y^{s}\right) \subseteq f_{Z}(x)-\mathbb{R}_{+}^{2} \backslash\{0\},
$$

which is visualized in Figure 5. Hence, the set of decision robust [·/strictly] efficient solutions is a subset of $Y$. Furthermore, because of the transitivity of $\leqq$, every element in $Y$ that is not decision robust [./strictly] efficient is dominated by another element of $Y$. Since $Y$ and $Z$ are totally ordered with respect to $\leqq, f_{1}$ is strongly de-and $f_{2}$ is strongly increasing, Theorem 27 can be applied, leading to the conclusion that all $y \in Y$ are decision robust [./strictly] efficient. 


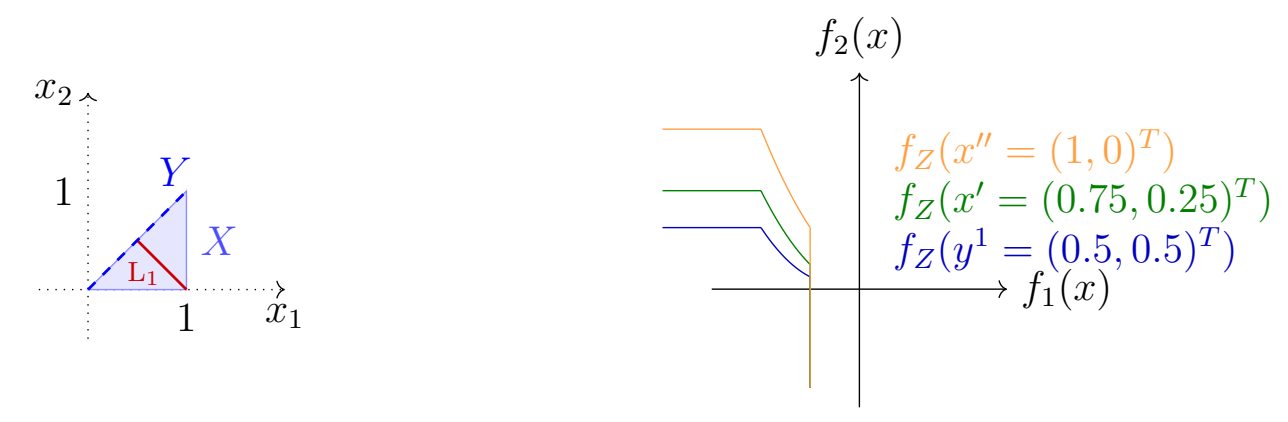

Figure 5: Illustration of Example 4. The sets $Y$ and $L_{s}$ for $s=1$ are illustrated on the left hand side. On the right hand side, inclusions of the sets $f_{Z}$ for different elements of $L_{1}$ are displayed. 


\section{B. The Robustness Gap for Uncertain Multiobjective Optimization}

C. Krüger, A. Schöbel, M. Wiecek 


\title{
The Robustness Gap for Uncertain Multiobjective Optimization
}

\author{
Corinna Krüger*1, Anita Schöbel ${ }^{1}$, and Margaret M. Wiecek ${ }^{\dagger 2}$ \\ ${ }^{1}$ Institute for Numerical and Applied Mathematics, University of \\ Goettingen, \\ Lotzestr. 16-18, 37083 Göttingen, Germany \\ ${ }^{2}$ Department of Mathematical Sciences, Clemson University, \\ Clemson, South Carolina 29634, USA
}

\begin{abstract}
In robust single-objective optimization, the robustness gap is a measure of the distance between the robust optimal objective value and the optimal objective values of the scenarios. While robust multiobjective optimization is a growing field of study, no notion of a robustness gap has been proposed. A concept of a robustness gap for uncertain multiobjective optimization problems is introduced. The gap is defined as the minimal distance between the robust Pareto set and the Pareto sets of the scenarios. Because the distance between Pareto sets is hard to compute, lower and upper bounds for the gap are derived for convex problems. It is shown that the gap is zero whenever the uncertainty is constraint-wise and objective-wise, supplementing a major result about the single-objective robustness gap. For linear problems, a condition for the gap to be zero is presented and a DC optimization problem is formulated to compute the lower bound.
\end{abstract}

\section{Introduction}

Uncertain multiobjective optimization addresses decision making problems with multiple and conflicting objectives, and uncertainties carried in parameters or decision variables. One of the paradigms to deal with this type of problems originates from robust optimization that has first been developed for uncertain single-objective optimization problems [BTGN09]. For a survey of recent literature on robust multiobjective optimization see [IS16] and [WD16].

\footnotetext{
*Supported by DFG RTG 1703 "Resource Efficiency in Corporate Networks".

${ }^{\dagger}$ Partially supported by grant N00014-16-1-2725 funded by the United States Office of Naval Research.
} 
There exist various concepts of robust solutions to uncertain multiobjective optimization problems (MOPs) and for each concept the corresponding robust solutions are defined as the solution set to a robust counterpart. A robust counterpart of an uncertain problem is a deterministic problem that typically takes into account worst case realizations of uncertain parameters or variables. So far, different concepts of robustness for MOPs have been developed, each of which can be associated with the solution set to a specific robust counterpart. The individual robust counterpart in each of [KL12, FW14, GJLVP14, Chu16] is a deterministic MOP, whereas in [EKS17, EIS14] the respective robust counterparts can be interpreted as set-valued optimization problems. Robustness concepts that lead to a multiobjective (vector-valued) robust counterpart are most widely studied. Moreover, under certain conditions, the set-valued problems studied as robust counterparts can be considered and solved as vector-valued problems, see, [EKS17] for the case of linear objective functions or [EIS14] for specific, so-called objective-wise, uncertainty sets.

Robust single-objective optimization resolves uncertainty by yielding an optimal solution to the robust counterpart and the objective value of that solution is worse than or equal to the optimal objective values of the scenarios. In order to evaluate the quality of a robust optimal solution in single-objective optimization, it is of interest to recognize and gauge the gap between its objective value and the optimal objective values of the scenarios, or in other words, to measure the loss when the robust optimal solution rather than an optimal solution to a scenario is implemented. This question of gap quantification, which is important to both mathematicians and practitioners, was initially raised in [BTN98] and [BTN99] who defined the gap as the minimal difference between the robust optimal objective value and the set of optimal objective values to the scenarios.

The question of how to measure a robustness gap in robust multiobjective optimization remains wide open because a gap of this type has not been defined. In this paper, we propose a definition of a robustness gap in uncertain multiobjective optimization with respect to a robustness concept whose robust counterpart can be considered an MOP and which is also the most often studied multiobjective robustness concept in the literature. Aiming to measure the proposed gap, we derive lower and upper bounds for the gap that are easier to calculate than the gap itself. We also investigate conditions under which the presented bounds are tight.

The paper is structured as follows. We continue this section by formulating the relevant MOPs and accompanying concepts, while in Section 2 we define the robustness gap for general uncertain MOPs. In Section 3, for convex MOPs, we derive bounds for this gap and give a sufficient condition for the zero gap. For uncertain linear MOPs we obtain further results in Section 4. Among others, we present another sufficient condition for the zero robustness gap and a DC optimization problem whose optimal objective value yields the lower bound on the robustness gap.

Throughout this work, let $\mathbb{R}^{p}$ be the $p$-dimensional real vector space. Let $\|\cdot\|: \mathbb{R}^{p} \rightarrow \mathbb{R}$ 
denote a norm on $\mathbb{R}^{p}$ and $\|\cdot\|_{*}$ denote the dual (or polar) norm to $\|\cdot\|$. In specific results we consider $\mathfrak{p}$-norms with $\mathfrak{p} \in[1, \infty]$ and block norms because of the properties they and their dual norms have. Given the norms, we define the primal space $\left(\mathbb{R}^{p},\|\cdot\|\right)$ and its dual space $\left(\mathbb{R}^{p},\|\cdot\|_{*}\right)$. To keep notation short, we also refer to the primal space $\left(\mathbb{R}^{p},\|\cdot\|\right)$ simply as $\mathbb{R}^{p}$.

For every point $\tilde{y} \in \mathbb{R}^{p}$, the closed and open ball of radius $\varepsilon>0$ are defined as $B(\tilde{y}, \varepsilon):=$ $\left\{y \in \mathbb{R}^{p} \mid\|y-\tilde{y}\| \leq \varepsilon\right\}$ and $B^{\circ}(\tilde{y}, \varepsilon):=\left\{y \in \mathbb{R}^{p} \mid\|y-\tilde{y}\|<\varepsilon\right\}$ respectively. For a set $A \subseteq \mathbb{R}^{p}$, the closure, the interior, and the relative interior of $A$ are denoted as $\operatorname{cl}(A)$, int $(A)$, and rel int $(A)$, respectively. The cone defined by the nonnegative orthant of $\mathbb{R}^{p}$ is $\mathbb{R}_{\geqq}^{p}:=\left\{y \in \mathbb{R}^{p} \mid y_{i} \geq 0 \forall i=1, \ldots, p\right\}$ and we also refer to $\mathbb{R}_{\geq}^{p}:=\mathbb{R}_{\geqq}^{p} \backslash\{0\}$ and $\mathbb{R}_{>}^{p}:=\operatorname{int}\left(\mathbb{R}_{\geqq}^{p}\right)$. For all $y, z \in \mathbb{R}^{p}$, where $p \geq 2$, the order relations induced by $\mathbb{R}_{\geqq}^{p}$ are given by $y<z \Leftrightarrow y_{i}<z_{i} \forall 1 \leq i \leq p ; y \leq z \Leftrightarrow y \neq z$ and $y_{i} \leq z_{i} \forall 1 \leq i \leq p$; and $y \leqq z \Leftrightarrow$ $y_{i} \leq z_{i} \forall 1 \leq i \leq p$.

We consider the uncertain multiobjective optimization problem

$$
\left\{\begin{array}{lll}
(\mathrm{P}(\xi)) & \min _{x} & f(x, \xi) \\
& \text { s. t. } & F(x, \xi) \geqq 0 \\
& & x \in \mathbb{R}^{n}
\end{array}\right\}_{\xi \in \mathcal{U}} .
$$

The uncertainty set $\mathcal{U}$ is assumed to be a compact subset of a finite dimensional real vector space and the elements $\xi \in \mathcal{U}$ are called scenarios of $\mathcal{U}$. Note that the uncertainty set is not assumed to be convex anywhere in the paper except for Section 3.3 and Section 4. For a fixed scenario $\xi \in \mathcal{U}$, it is assumed that no uncertainty is present in $\mathrm{P}(\xi)$, i.e., all data of $\mathrm{P}(\xi)$ is known. Therefore, for each $\xi \in \mathcal{U}, \mathrm{P}(\xi)$ is considered a deterministic MOP.

We make the following Continuity Assumption: The functions $f(\cdot, \xi): \mathbb{R}^{n} \rightarrow \mathbb{R}^{p}$ and $F(\cdot, \xi): \mathbb{R}^{n} \rightarrow \mathbb{R}^{m}$ are assumed to be continuous for all $\xi \in \mathcal{U}$ and the functions $f(x, \cdot): \mathcal{U} \rightarrow$ $\mathbb{R}^{p}$ and $F(x, \cdot): \mathcal{U} \rightarrow \mathbb{R}^{m}$ are assumed to be continuous for all $x \in \mathbb{R}^{n}$.

For every scenario $\xi \in \mathcal{U}$, the feasible set of $\mathrm{P}(\xi)$ is denoted as

$$
X(\xi):=\left\{x \in \mathbb{R}^{n} \mid F(x, \xi) \geqq 0\right\} .
$$

and it is assumed that $X(\xi)$ is compact. Also, for every $\xi \in \mathcal{U}$, the outcome set of $\mathrm{P}(\xi)$, which is the image set of the feasible set $X(\xi)$, is given as

$$
\mathcal{Y}(\xi):=\left\{y \in \mathbb{R}^{p} \mid \exists x \in X(\xi): y=f(x, \xi)\right\}=f(X(\xi), \xi) .
$$

The set $\mathcal{Y}(\xi)$ is compact since $X(\xi)$ is assumed to be compact and $f(\cdot, \xi)$ is assumed to be continuous.

The classical solution concept for deterministic MOPs is (Pareto-)efficiency, which is defined as follows with respect to the deterministic $\operatorname{MOP} \mathrm{P}(\xi)$ for a fixed scenario $\xi \in \mathcal{U}$. 
Definition 1. An outcome point $y^{*} \in \mathcal{Y}(\xi)$ is called a Pareto point for $\mathrm{P}(\xi)$ if there is no $y \in \mathcal{Y}(\xi)$ with the property $y \in\left\{y^{*}\right\}-\mathbb{R}_{\geq}^{p}$ or, equivalently, if there is no $y \in \mathcal{Y}(\xi)$ with the property $y \leq y^{*}$. A feasible solution $x^{*} \in X(\xi)$ is called a (Pareto-)efficient solution for $\mathrm{P}(\xi)$ if the image $f\left(x^{*}, \xi\right)$ is a Pareto point of $\mathrm{P}(\xi)$.

The (Pareto-)efficient set of $\mathrm{P}(\xi)$ is denoted as $X_{E}(\xi)$, and the Pareto set of $\mathrm{P}(\xi)$ is denoted as $\mathcal{Y}_{P}(\xi)$.

For defining the feasibility of the uncertain multiobjective problem $\{\mathrm{P}(\xi)\}_{\xi \in \mathcal{U}}$, the robustness concepts we take into account use the definition of robust feasibility by [BTGN09], which is the most often used concept in robust optimization.

Definition 2. A point $x^{*} \in \mathbb{R}^{n}$ is called a robust feasible solution to $\{\mathrm{P}(\xi)\}_{\xi \in \mathcal{U}}$ if $x^{*}$ is feasible for all possible realizations of uncertainty, i.e., if

$$
x^{*} \in X^{\mathrm{RC}}:=\left\{x \in \mathbb{R}^{n} \mid F(x, \xi) \geqq 0 \forall \xi \in \mathcal{U}\right\} .
$$

The set $X^{\mathrm{RC}}$ is called the robust feasible set.

As a consequence of (1) and Definition 2, we obtain

$$
X^{\mathrm{RC}}=\bigcap_{\xi \in \mathcal{U}} X(\xi)
$$

Hence, the robust feasible set $X^{\mathrm{RC}}$ is compact as an intersection of compact sets.

In this study we address multiobjective robustness concepts that are characterized by a robust counterpart that assumes the form of an MOP over the feasible set $X^{\mathrm{RC}}$. We therefore formulate the general multiobjective robust counterpart for $\{\mathrm{P}(\xi)\}_{\xi \in \mathcal{U}}$ as

$$
\begin{array}{ll}
\text { (RC) } \quad \min _{x} & f^{\mathrm{RC}}(x) \\
\text { s. t. } & x \in X^{\mathrm{RC}},
\end{array}
$$

where $f^{\mathrm{RC}}: \mathbb{R}^{n} \rightarrow \mathbb{R}^{p}$ is a deterministic function that is defined depending on the concept of robustness. The most frequently used choice for the objective function $f^{\mathrm{RC}}$ of $\mathrm{RC}$ is given as

$$
f_{i}^{\mathrm{RC}}(x)=\sup _{\xi \in \mathcal{U}} f_{i}(x, \xi)
$$

for all $1 \leq i \leq p$ and for any $x \in \mathbb{R}^{n}$. For studies of the robust counterpart corresponding to this kind of objective function, see, e.g., [KL12, FW14, GJLVP14]. There are also various investigations of uncertain MOPs where the uncertainty does not affect the objective functions at all, leading to an $\mathrm{RC}$ with the same deterministic objective function as the original problem, see, e.g., [Chu16, DKW12]. Note that the latter type of RC can be considered as a special case of the former type of $\mathrm{RC}$ because, if the objective function is not affected by uncertainty, the supremum over all scenarios of the objective function is the objective 
function itself. Therefore, in the following, we assume that the objective function of RC is given by (4).

The robust outcome set, which is the image of the robust feasible set $X^{\mathrm{RC}}$, is denoted as

$$
\mathcal{Y}^{\mathrm{RC}}:=\left\{f^{\mathrm{RC}}(x) \mid x \in X^{\mathrm{RC}}\right\}=f^{\mathrm{RC}}\left(X^{\mathrm{RC}}\right) .
$$

Because the robust counterpart $\mathrm{RC}$ is a deterministic MOP, we can apply to it the traditional solution concepts known for MOPs, see Definition 1.

Definition 3. A point $z^{*} \in \mathcal{Y}^{\mathrm{RC}}$ is called a robust Pareto point of $\{\mathrm{P}(\xi)\}_{\xi \in \mathcal{U}}$ if it is a Pareto point of $\mathrm{RC}$ in the sense of Definition 1, i.e., if there is no $z \in \mathcal{Y}^{\mathrm{RC}}$ with the property $z \in\left\{z^{*}\right\}-\mathbb{R}_{\geq}^{p}$. A solution $x^{*} \in X^{\mathrm{RC}}$ is called a robust (Pareto-)efficient solution of $\{\mathrm{P}(\xi)\}_{\xi \in \mathcal{U}}$ if it is an efficient solution to $\mathrm{RC}$ in the sense of Definition 1.

The robust (Pareto-)efficient set of $\{\mathrm{P}(\xi)\}_{\xi \in \mathcal{U}}$ is denoted as $X_{E}^{\mathrm{RC}}$, and the robust Pareto set of $\{\mathrm{P}(\xi)\}_{\xi \in \mathcal{U}}$ is denoted as $\mathcal{Y}_{P}^{\mathrm{RC}}$. We remark that robust efficiency according to Definition 3 is often called point-based minmax robust efficiency to distinguish it from other minmax robust efficiency concepts such as set-based or hull-based robust efficiency, see [EIS14, BF17].

We also use the general notion of the ideal point $z_{\mathrm{RC}}^{I}$ of $\mathrm{RC}$, which is given by $\left(z_{\mathrm{RC}}^{I}\right)_{i}:=$ $\min _{x \in X^{\mathrm{RC}}} f_{i}^{\mathrm{RC}}(x)$ for each component $1 \leq i \leq p$, where $f_{i}^{\mathrm{RC}}: \mathbb{R}^{n} \rightarrow \mathbb{R}$ is the $i$-th component of the objective function $f^{\mathrm{RC}}$ of $\mathrm{RC}$.

We conclude the section by presenting a result for the outcome set of $\mathrm{RC}$ and we also see that the special case of $\{\mathrm{P}(\xi)\}_{\xi \in \mathcal{U}}$ having a deterministic objective functions, i.e., $f=f^{\mathrm{RC}}$, leads to a stronger result.

Proposition 4. Let $f^{\mathrm{RC}}: \mathbb{R}^{n} \rightarrow \mathbb{R}^{p}$ be such that for all $1 \leq i \leq p$ and $x \in \mathbb{R}^{n}$ it holds $f_{i}^{\mathrm{RC}}(x)=\sup _{\xi \in \mathcal{U}} f_{i}(x, \xi)$. Then

$$
\mathcal{Y}^{\mathrm{RC}} \subseteq \bigcap_{\xi \in \mathcal{U}} \mathcal{Y}(\xi)+\mathbb{R}_{\geqq}^{p}
$$

and, if $f=f^{\mathrm{RC}}$, then

$$
\mathcal{Y}^{\mathrm{RC}} \subseteq \bigcap_{\xi \in \mathcal{U}} \mathcal{Y}(\xi)
$$

Proof. If $f^{\mathrm{RC}}=f$ for all $x \in \mathbb{R}^{n}$ and $1 \leq i \leq p$, then Equation (2) directly results in

$$
\mathcal{Y}^{\mathrm{RC}}=f\left(X^{\mathrm{RC}}\right)=f\left(\bigcap_{\xi \in \mathcal{U}} X(\xi)\right) \subseteq \bigcap_{\xi \in \mathcal{U}} f(X(\xi))=\bigcap_{\xi \in \mathcal{U}} \mathcal{Y}(\xi) .
$$

Hence, let $f_{i}^{\mathrm{RC}}(x)=\sup _{\xi \in \mathcal{U}} f_{i}(x, \xi)$ for all $x \in \mathbb{R}^{n}$ and $1 \leq i \leq p$ and let $y \in \mathcal{Y}^{\mathrm{RC}}$. Then there exists $x \in X^{\mathrm{RC}}$ such that $y=f^{\mathrm{RC}}(x)$ and consequently,

$$
f_{i}(x, \xi) \leq \sup _{\xi^{\prime} \in \mathcal{U}} f_{i}\left(x, \xi^{\prime}\right)=f_{i}^{\mathrm{RC}}(x)=y_{i}
$$


for all $\xi \in \mathcal{U}$ and $1 \leq i \leq p$. Hence, $y \in\{f(x, \xi)\}+\mathbb{R}_{\geqq}^{p}$ for all $\xi \in \mathcal{U}$. Because $x \in X^{\mathrm{RC}}=$ $\bigcap_{\xi \in \mathcal{U}} X(\xi)$, we have $f(x, \xi) \in \mathcal{Y}(\xi)$ for all $\xi \in \mathcal{U}$. Consequently, $y \in\{f(x, \xi)\}+\mathbb{R}_{\geqq}^{p} \subseteq$ $\mathcal{Y}(\xi)+\mathbb{R}_{\geqq}^{p}$ for all $\xi \in \mathcal{U}$.

The following result is a direct consequence of Proposition 4.

Corollary 5. Let $f^{\mathrm{RC}}: \mathbb{R}^{n} \rightarrow \mathbb{R}^{p}$ be such that for all $1 \leq i \leq p$ and $x \in \mathbb{R}^{n}$ it holds $f_{i}^{\mathrm{RC}}(x)=\sup _{\xi \in \mathcal{U}} f_{i}(x, \xi)$. Then for very $z \in \mathcal{Y}_{P}^{\mathrm{RC}}$ and for every $\xi \in \mathcal{U}$, there exists $y \in \mathcal{Y}_{P}(\xi)$ such that $y \in\{z\}-\mathbb{R}_{\geqq}^{p}$.

Proof. Let $z \in \mathcal{Y}_{P}^{\mathrm{RC}}$ and $\xi \in \mathcal{U}$. By Proposition 4 , there exists $y^{\prime} \in \mathcal{Y}(\xi)$ such that $y^{\prime} \leqq z$. Then there exists $y \in \mathcal{Y}_{P}(\xi)$ such that $y \leqq y^{\prime}$ according to [Luc84, Prop. 3.1] because $\overline{\mathcal{Y}}(\xi)$ is compact, and we obtain $y \leqq y^{\prime} \leqq z$.

\section{Defining a Robustness Gap for Uncertain Multiob- jective Optimization}

In this section we first review the definition of the robustness gap in single-objective optimization and then propose a definition for the multiobjective case.

Let $g: \mathbb{R}^{n} \times \mathcal{U} \rightarrow \mathbb{R}$. For the uncertain single-objective optimization problem formulated as

$$
\left\{\left(P^{\mathrm{s}-\mathrm{o}}(\xi)\right) \min _{x \in X(\xi)} g(x, \xi)\right\}_{\xi \in \mathcal{U}}
$$

its robust counterpart is defined as

$$
\left(R C^{\mathrm{s}-\mathrm{o}}\right) \min _{x \in X^{\mathrm{RC}}} g^{\mathrm{RC}}(x),
$$

where $g^{\mathrm{RC}}: \mathbb{R}^{n} \rightarrow \mathbb{R}$ with $g^{\mathrm{RC}}(x):=\sup _{\xi \in \mathcal{U}} g(x, \xi)$ is a deterministic scalar-valued function. The following definition of the single-objective robustness gap $\vartheta^{\text {s-o }}$ goes back to [BTN98].

Definition 6. The single-objective robustness gap is defined as

$$
\vartheta^{s-o}:=\min _{x \in X^{\mathrm{RC}}} g^{\mathrm{RC}}(x)-\sup _{\xi \in \mathcal{U}} \min _{x \in X(\xi)} g(x, \xi) .
$$

The concept in Definition 6 aims to evaluate decision maker's loss when choosing a robust optimal solution. The knowledge of the magnitude of this loss may help decision makers to decide whether they would accept this solution. If there is a scenario $\xi \in \mathcal{U}$ such that the optimal objective value of $\left(P^{\mathrm{s}-\mathrm{o}}(\xi)\right)$ is equal to the robust optimal objective value, then the robustness gap is zero and the decision maker might be likely to accept a robust optimal solution as the preferred solution. The knowledge that the gap is nonzero but small may also encourage the decision maker to accept a robust optimal solution. If the gap is large, 
the decision maker will be aware of the loss and in this way motivated to look for a less conservative decision. In general, the robustness gap is a problem intrinsic characteristic; its size depends on the structure of the optimization problem, the uncertainty set, and the way both structures interact.

The single-objective robustness gap according to Definition 6 is illustrated in the next example.

Example 7. Consider the following uncertain single-objective optimization problem

$$
\left\{\begin{array}{lll}
\left(P^{s-o}(\xi)\right) \quad \min _{x} & \xi_{1} x_{1}+\xi_{2} x_{2} \\
\text { s. } t . & x \in X(\xi)
\end{array}\right\}_{\xi \in \mathcal{U}},
$$

where

$$
X(\xi):=\left\{x \in \mathbb{R}^{2} \mid 3 x_{1}+x_{2} \geq \xi_{3} \wedge x_{1}+4 x_{2} \geq \xi_{4} \wedge-4 x_{1}-5 x_{2} \geq \xi_{5}\right\}
$$

for all $\xi \in \mathcal{U}$ and $\mathcal{U}=\left\{\xi^{1}:=(2,3,14,12,-48)^{T}, \xi^{2}:=(4,1,11,22,-55)^{T}\right\} \subseteq \mathbb{R}^{5}$. Hence, the set of robust feasible solutions is

$$
X^{\mathrm{RC}}=\left\{x \in \mathbb{R}^{2} \mid 3 x_{1}+x_{2} \geq 14 \wedge x_{1}+4 x_{2} \geq 22 \wedge-4 x_{1}-5 x_{2} \geq-48\right\}
$$

and the robust counterpart of $\left\{\left(P^{s-o}(\xi)\right)\right\}_{\xi \in \mathcal{U}}$ is

(RC) $\quad \min _{x} \max \left\{2 x_{1}+3 x_{2}, 4 x_{1}+x_{2}\right\}$

$$
\text { s. t. } \quad x \in X^{\mathrm{RC}} \text {. }
$$

The single-objective robustness gap according to Definition 6 is

$$
\begin{aligned}
\vartheta^{s-o} & =\min _{x \in X^{\mathrm{RC}}} \max \left\{2 x_{1}+3 x_{2}, 4 x_{1}+x_{2}\right\}-\max \left\{\min _{x \in X\left(\xi^{1}\right)} 2 x_{1}+3 x_{2}, \min _{x \in X\left(\xi^{2}\right)} 4 x_{1}+x_{2}\right\} \\
& =20.25-\max \{14,11\}=6.25 .
\end{aligned}
$$

We now extend the notion of the robustness gap from uncertain single-objective optimization to uncertain MOPs. According to Definition 6, the single-objective robustness gap can be considered as the minimal distance between the robust optimal objective value and the set of the optimal objective values of all the scenarios $\xi \in \mathcal{U}$. In multiobjective optimization, the idea of a minimal distance between Pareto outcomes can be represented by the minimal distance between the robust Pareto set $\mathcal{Y}_{P}^{\mathrm{RC}}$ and the Pareto sets $\mathcal{Y}_{P}(\xi)$ of the scenarios $\xi \in \mathcal{U}$. We therefore introduce the following definition of a robustness gap for uncertain MOPs.

Definition 8. For each $\xi \in \mathcal{U}$ let

$$
\vartheta(\xi):=\operatorname{dist}\left(\mathcal{Y}_{P}^{\mathrm{RC}}, \mathcal{Y}_{P}(\xi)\right)=\inf _{z \in \mathcal{Y}_{P}^{\mathrm{RC}}} \inf _{y \in \mathcal{Y}_{P}(\xi)}\|z-y\|
$$

and define the multiobjective robustness gap as

$$
\vartheta:=\inf _{\xi \in \mathcal{U}} \vartheta(\xi)=\inf _{\xi \in \mathcal{U}} \operatorname{dist}\left(\mathcal{Y}_{P}^{\mathrm{RC}}, \mathcal{Y}_{P}(\xi)\right) .
$$



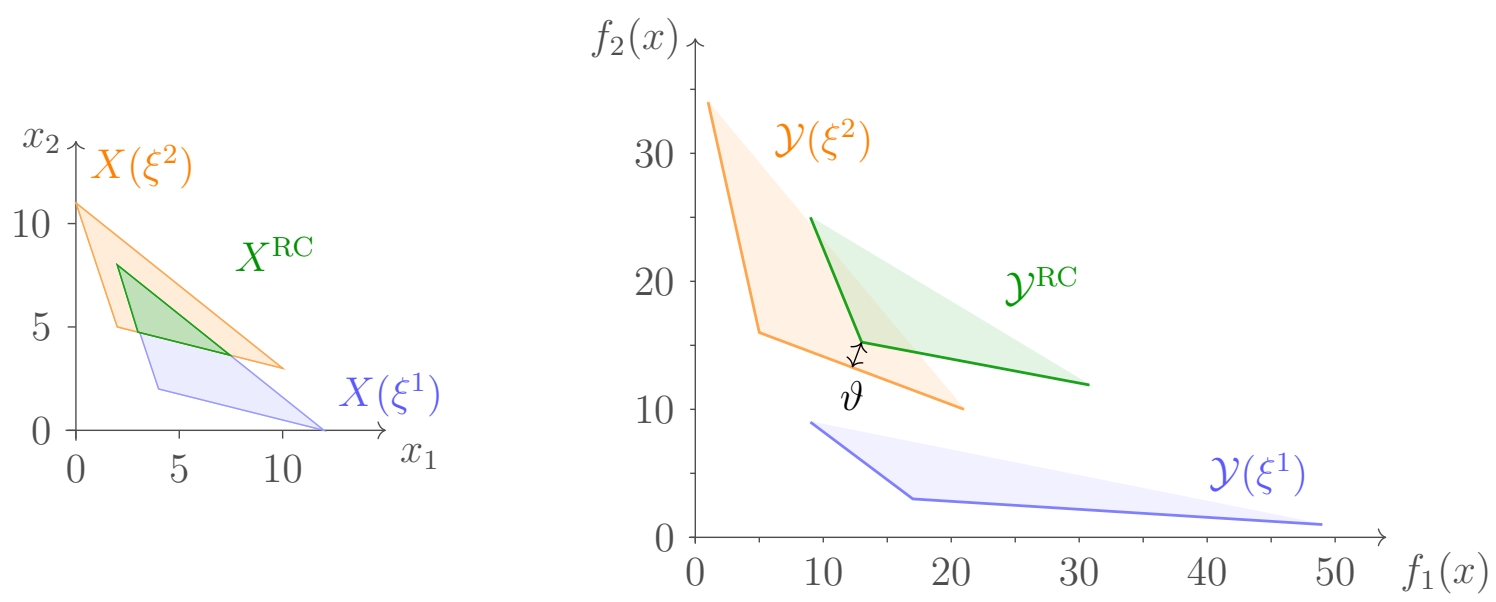

Figure 1: Illustration of Example 9. The feasible sets are displayed on the left hand side and the outcome sets are displayed on the right hand side. In the right hand picture, the thick solid lines depict the Pareto sets $\mathcal{Y}_{P}\left(\xi^{1}\right), \mathcal{Y}_{P}\left(\xi^{2}\right)$ and $\mathcal{Y}_{P}^{\mathrm{RC}}$ and $\vartheta$ illustrates the robustness gap.

The multiobjective robustness gap $\vartheta$ depends on the norm $\|\cdot\|$ of the primal space $\left(\mathbb{R}^{p},\|\cdot\|\right)$. The gap is illustrated in Example 9 and Figure 1 for the Euclidean norm.

Example 9. We investigate a multiobjective version of the problem studied in Example 7 and consider the following uncertain MOP

$$
\left\{\begin{array}{ccc}
(P(\xi)) & \min _{x} & \left(\xi_{1} x_{1}+1, \xi_{2} x_{2}+1\right)^{T} \\
\text { s. } t . & x \in X(\xi)
\end{array}\right\}_{\xi \in \mathcal{U}}
$$

where $\mathcal{U}=\left\{\xi^{1}:=(2,3,14,12,-48)^{T}, \xi^{2}:=(4,1,11,22,-55)^{T}\right\} \subseteq \mathbb{R}^{5}$. The corresponding robust counterpart is

(RC) $\quad \min _{x}\left(\max _{\xi \in \mathcal{U}} \xi_{1} x_{1}+1, \max _{\xi \in \mathcal{U}} \xi_{2} x_{2}+1\right)^{T}$

$$
\text { s. } t \text {. } \quad x \in X^{\mathrm{RC}} \text {. }
$$

Assuming the Euclidean norm in the objective space $\mathbb{R}^{2}$, the multiobjective robustness gap is

$$
\vartheta=\min _{\xi \in \mathcal{U}} \min _{z \in \mathcal{Y}_{P}^{\mathrm{RC}}} \min _{y \in \mathcal{Y}_{P}(\xi)}\|z-y\|_{2}=\frac{18}{\sqrt{73}} \approx 2.11
$$

which is also illustrated in Figure 1.

Moreover, we note that the robustness gap, which depends on the structure of the unertainty set $\mathcal{U}$, would be zero if $\mathcal{U}$ was defined differently. For instance, for the uncertainty set $\mathcal{U}^{\prime}:=\left\{\xi \in \mathbb{R}^{5} \mid \xi_{i} \in\left[\xi_{i}^{1}, \xi_{i}^{2}\right], i=1, \ldots, 5\right\}$ with $\xi^{1}, \xi^{2} \in \mathcal{U}$ as defined before, the robustness gap is zero. 
However, in general robust multiobjective optimization, the robustness gap is hard to compute. By Definition 8, determining the robustness gap requires solving a minimization problem with decision variables that are elements of various Pareto sets, which are generally nonconvex even for convex MOPs. For this reason, in the next section, we consider convex problems for which we derive a lower bound as well as an upper bound on the robustness gap $\vartheta$.

\section{Bounds on the Robustness Gap for Convex Prob- lems}

In this section, we introduce convexity assumptions and we develop upper and lower bounds for the multiobjective robustness gap. We make use of a scalarization of MOPs whose scalarizing parameter turns out to be instrumental in the derivation of the bounds.

The following Convexity Assumption is made: In the remainder of this work, we require that $f(\cdot, \xi): \mathbb{R}^{n} \rightarrow \mathbb{R}^{p}$ be $\mathbb{R}_{\geq}^{p}$-convex and $F(\cdot, \xi): \mathbb{R}^{n} \rightarrow \mathbb{R}^{m}$ be $\mathbb{R}_{\geq}^{m}$-concave for all $\xi \in \mathcal{U}$, which implies that each instance of $\{\mathrm{P}(\xi)\}_{\xi \in \mathcal{U}}$ is a convex MOP.

Under this assumption, the feasible sets $X(\xi)$ are convex and their images $\mathcal{Y}(\xi)$ are $\mathbb{R}_{\geqq}^{p}$ convex for all $\xi \in \mathcal{U}$ and, consequently, the robust feasible set $X^{\mathrm{RC}}=\bigcap_{\xi \in \mathcal{U}} X(\xi)$ is convex as an intersection of convex sets. Under the Convexity Assumption on $f(\cdot, \xi)$ for all $\xi \in \mathcal{U}$, the function $f^{\mathrm{RC}}$ as given in (4) is $\mathbb{R}_{\geqq}^{p}$-convex as the component-wise supremum of convex functions. Because $f^{\mathrm{RC}}$ is $\mathbb{R}^{p}$-convex and $X^{\mathrm{RC}}$ is convex, $\mathrm{RC}$, see (3), is a convex problem. $\mathbb{R}_{\geqq}^{p}$-convexity of $f^{\mathrm{RC}}$ implies that $\mathcal{Y}^{\mathrm{RC}}=f^{\mathrm{RC}}\left(X^{\mathrm{RC}}\right)$ is an $\mathbb{R}_{\geqq}^{p}$-convex set. Furthermore, $f^{\mathrm{RC}}$ is continuous because it is $\mathbb{R}_{\geq}^{p}$-convex and therefore $\mathcal{Y}^{\mathrm{RC}}$ is compact as the image of the compact set $X^{\mathrm{RC}}$ under $f^{\mathrm{RC}}$.

Note that if $f_{i}$ is strictly convex for all $1 \leq i \leq p$, then $f_{i}^{\mathrm{RC}}$ as given in (4) is strictly convex for all $1 \leq i \leq p$ and we obtain that the efficient sets $X_{E}^{\mathrm{RC}}$ and $X_{E}(\xi)$ are compact for all $\xi \in \mathcal{U}$, see [BS99, Th. 3.4]. The images of these efficient sets, $\mathcal{Y}_{P}^{\mathrm{RC}}$ and $\mathcal{Y}_{P}(\xi)$ for all $\xi \in \mathcal{U}$, are then compact due to the continuity of the respective objective functions. Given the compactness, the minimum distance between each pair of Pareto sets is attained and the multiobjective robustness gap according to Definition 8 is $\vartheta=\inf _{\xi \in \mathcal{U}} \min _{z \in \mathcal{Y}_{P}^{\mathrm{RC}}} \min _{y \in \mathcal{Y}_{P}(\xi)}\|z-y\|$.

Since every instance of $\{\mathrm{P}(\xi)\}_{\xi \in \mathcal{U}}$ and RC are convex MOPs, we are able to use the wellknown weighted-sum scalarization of MOPs, see [Geo68], to construct bounds on the robustness gap $\vartheta$. This scalarization transforms deterministic MOPs into single objective problems by applying linear functions to the objective functions of MOPs. Linear functions mapping the objective space $\left(\mathbb{R}^{p},\|\cdot\|\right)$ to $\mathbb{R}$ are typically elements of the space $\left(\mathbb{R}^{p},\|\cdot\|_{*}\right)$ that is dual to the primal space $\left(\mathbb{R}^{p},\|\cdot\|\right)$. In the dual space, the linear functions that are used for weighted-sum scalarization are exactly the elements of the cone $\mathbb{R}_{\geq}^{p} \subseteq\left(\mathbb{R}^{p},\|\cdot\|_{*}\right)$ that is the dual cone to the Pareto cone $\mathbb{R}_{\geqq}^{p} \subseteq\left(\mathbb{R}^{p},\|\cdot\|\right)$, see, e.g., [BGW09] and [Luc89]. 
In the following, bounds on the robustness gap $\vartheta$ are constructed by applying the weightedsum scalarization. Hence, the calculation of these bounds involves the dual norm $\|\cdot\|_{*}$ rather than the primal norm $\|\cdot\|$.

Applying the weighted-sum scalarization to the problems $\{\mathrm{P}(\xi)\}_{\xi \in \mathcal{U}}$ and RC, we obtain for each weight vector $\lambda \in \mathbb{R}_{\geqq}^{p} \subseteq\left(\mathbb{R}^{p},\|\cdot\|_{*}\right)$ the uncertain scalarized problem

$$
\left\{\begin{array}{lll}
(\mathrm{P}(\xi, \lambda)) \quad \min _{x} & \lambda^{T} f(x, \xi) \\
\text { s.t. } & x \in X(\xi)
\end{array}\right\}_{\xi \in \mathcal{U}},
$$

and the scalarized robust counterpart

$$
\begin{array}{lll}
(\mathrm{RC}(\lambda)) & \min _{x} & \lambda^{T} f^{\mathrm{RC}}(x) \\
\text { s. t. } & x \in X^{\mathrm{RC}} .
\end{array}
$$

Because the problems $(\mathrm{P}(\xi, \lambda))$ and $(\mathrm{RC}(\lambda))$ are single-objective, we can to apply to them the definition of the single-objective robustness gap in Definition 6 . Hence, we obtain

$$
\begin{aligned}
\min \left\{\lambda^{T} f^{\mathrm{RC}}(x) \mid x \in X^{\mathrm{RC}}\right\} & -\sup _{\xi \in \mathcal{U}} \min \left\{\lambda^{T} f(x, \xi) \mid x \in X(\xi)\right\} \\
= & \inf _{\xi \in \mathcal{U}}\left(\min _{z \in \mathcal{Y} \mathrm{RC}} \lambda^{T} z-\min _{y \in \mathcal{Y}(\xi)} \lambda^{T} y\right) .
\end{aligned}
$$

We then introduce a definition of a gap for a scenario $\xi$ and a weight vector $\lambda$, which allows us to define the lower robustness bound $\Delta^{L}$ and the upper robustness bound $\Delta^{U}$. Note that, because each weight vector $\lambda$ lives in the dual space $\left(\mathbb{R}^{p},\|\cdot\|_{*}\right)$, all $\lambda$ are normalized with respect to the dual norm.

Definition 10. For each $\xi \in \mathcal{U}$ and for each $\lambda \in \mathbb{R}_{\geqq \text {, we define }}^{p}$

$$
\Delta(\xi, \lambda):=\min _{z \in \mathcal{Y}^{\mathrm{RC}}} \lambda^{T} z-\min _{y \in \mathcal{Y}(\xi)} \lambda^{T} y
$$

Furthermore, we define the lower robustness bound and upper robustness bound as

$$
\Delta^{L}:=\inf _{\xi \in \mathcal{U}} \min _{\substack{\lambda \in \mathbb{R}_{\geq}^{p},\|\lambda\|_{*}=1}} \Delta(\xi, \lambda) \quad \text { and } \quad \Delta^{U}:=\inf _{\xi \in \mathcal{U}} \max _{\substack{\lambda \in \mathbb{R}_{\geq}^{p},\|\lambda\|_{*}=1}} \Delta(\xi, \lambda) .
$$

Note that, in single-objective optimization, it holds $\left\{\lambda \in \mathbb{R}_{>} \mid\|\lambda\|_{*}=1\right\}=\{1\}$. Therefore, $\vartheta^{\text {s-o }}=\Delta^{L}=\Delta^{U}$ in uncertain single-objective optimization which additionally justifies Definition 10. Also note that, in the multiobjective setting, $\Delta^{L}$ and $\Delta^{U}$ are not only likely to be different but that they are also dependent on the dual norm $\|\cdot\|_{*}$. From Definition 10 we immediately obtain that $\Delta^{L}$ and $\Delta^{U}$ are nonegative. 
Proposition 11. For $\Delta^{L}$ and $\Delta^{U}$ as defined in Definition 10 holds

$$
0 \leq \Delta^{L} \leq \Delta^{U}
$$

Proof. Based on the properties of the weighted-sum scalarization, see, e.g., [Geo68], we have for all $\lambda \in \mathbb{R}_{\geq}^{p}$ and for all $\xi \in \mathcal{U}$

$$
\min _{y \in \mathcal{Y}(\xi)} \lambda^{T} y=\min _{y \in \mathcal{Y}(\xi)+\mathbb{R}_{\geqq}^{p}} \lambda^{T} y \leq \min _{y \in \mathcal{Y}^{\mathrm{RC}}} \lambda^{T} y
$$

where the inequality results from Proposition 4.

The lower robustness bound $\Delta^{L}$ and the upper robustness bound $\Delta^{U}$ for Example 9 are calculated in Example 12.

Example 12. We consider the uncertain MOP studied in Example 9 for which we determine $\Delta^{L}$ and $\Delta^{U}$. In order to calculate $\Delta^{L}$, we consider $\tilde{z}:=(9,25)^{T} \in \mathcal{Y}^{\mathrm{RC}}, \tilde{y}:=(9,9)^{T} \in$ $\mathcal{Y}\left(\xi^{1}\right)$ and $\tilde{\lambda}:=(1,0)^{T} \in \mathbb{R}_{>}^{2}$ and since, in this example, the primal and the dual space are both $\left(\mathbb{R}^{2},\|\cdot\|_{2}\right)$, we note $\|\bar{\lambda}\|_{2}=1$. Then

$$
0 \leq \Delta^{L}=\inf _{\xi \in \mathcal{U}} \min _{\substack{\lambda \in \mathbb{R}_{\geq}^{2},\|\lambda\|_{2}=1}}\left(\min _{x \in \mathcal{Y}^{\mathrm{RC}}} \lambda^{T} z-\min _{y \in \mathcal{Y}(\xi)} \lambda^{T} y\right) \leq \Delta\left(\xi^{1}, \tilde{\lambda}\right) \leq \tilde{\lambda}^{T} \tilde{z}-\tilde{\lambda}^{T} \tilde{y}=9-9=0,
$$

where the first inequality results from Proposition 11. Consequently, we obtain $\Delta^{L}=0$ and the lower robustness bound is displayed in Figure 2.

In order to determine $\Delta^{U}$, we first calculate $\max _{\substack{\lambda \in \mathbb{R}_{\geq}^{2},\|\lambda\|_{2}=1}} \Delta\left(\xi^{2}, \lambda\right)$. By linear programming theory, for any $\lambda \in \mathbb{R}_{\geq}^{2}$, the sets $\arg \min _{z \in \mathcal{Y}^{\mathrm{RC}}} \lambda^{T} z$ and $\arg \min _{y \in \mathcal{Y}\left(\xi^{2}\right)} \lambda^{T} y$ each contain at least one vertex of $\mathcal{Y}^{\overline{\mathrm{RC}}}$ and $\mathcal{Y}\left(\xi^{2}\right)$ respectively. Hence, we can write $\Lambda:=\left\{\lambda \in \mathbb{R}_{\geq}^{2} \mid\right.$ $\left.\|\lambda\|_{2}=1\right\}$ as a union of subsets $\Lambda=\bigcup_{i=1}^{5} \Lambda_{i}$ such that $\bigcap_{\lambda \in \Lambda_{i}} \arg \min _{z \in \mathcal{Y}^{\mathrm{RC}}} \lambda^{T} z \neq \emptyset$ and $\bigcap_{\lambda \in \Lambda_{i}} \arg \min _{y \in \mathcal{Y}\left(\xi^{2}\right)} \lambda^{T} y \neq \emptyset$ for all $i=1, \ldots, 5$. To this end, we distinguish the five cases $\lambda \in \Lambda_{1}, \cdots, \Lambda_{5}$, where $\Lambda_{1}:=\left\{\lambda \in \Lambda \mid \lambda_{2} \leq \frac{9}{2} \lambda_{1} \leq 1\right\}, \Lambda_{2}:=\left\{\lambda \in \Lambda \mid \frac{39}{16} \lambda_{2} \leq \lambda_{1} \leq \frac{9}{2} \lambda_{2}\right\}$, $\Lambda_{3}:=\left\{\lambda \in \Lambda \mid \frac{3}{8} \lambda_{2} \leq \lambda_{1} \leq \frac{39}{16} \lambda_{2}\right\}, \Lambda_{4}:=\left\{\lambda \in \Lambda \mid \frac{3}{16} \lambda_{2} \leq \lambda_{1} \leq \frac{3}{8} \lambda_{2}\right\}$ and $\Lambda_{5}:=\{\lambda \in \Lambda \mid$ $\left.0 \leq \lambda_{1} \leq \frac{3}{16} \lambda_{1}\right\}$. By direct calculation, we then obtain

$$
\max _{\substack{\lambda \in \mathbb{R}_{\geq}^{2},\|\lambda\|_{2}=1}} \Delta\left(\xi^{2}, \lambda\right)=\max _{i=1, \ldots, 5}\left\{\max _{\lambda \in \Lambda_{i}} \Delta\left(\xi^{2}, \lambda\right)\right\}=\Delta\left(\xi^{2},(1,0)^{T}\right)=8 .
$$

Next, we show $\max _{\substack{\lambda \in \mathbb{R}_{\geq}^{2},\|\lambda\|_{2}=1}} \Delta\left(\xi^{2}, \lambda\right)<\max _{\substack{\lambda \in \mathbb{R}_{\geq}^{2},\|\lambda\|_{2}=1}} \Delta\left(\xi^{1}, \lambda\right)$. For $\lambda^{\prime}:=(0,1)^{T}$ we obtain

$$
\max _{\substack{\lambda \in \mathbb{R}_{\geq}^{2},\|\lambda\|_{2}=1}} \Delta\left(\xi^{1}, \lambda\right) \geq \Delta\left(\xi^{1}, \lambda^{\prime}\right)=13-1=12>8=\max _{\substack{\lambda \in \mathbb{R}_{\geq}^{2},\|\lambda\|_{2}=1}} \Delta\left(\xi^{2}, \lambda\right) .
$$




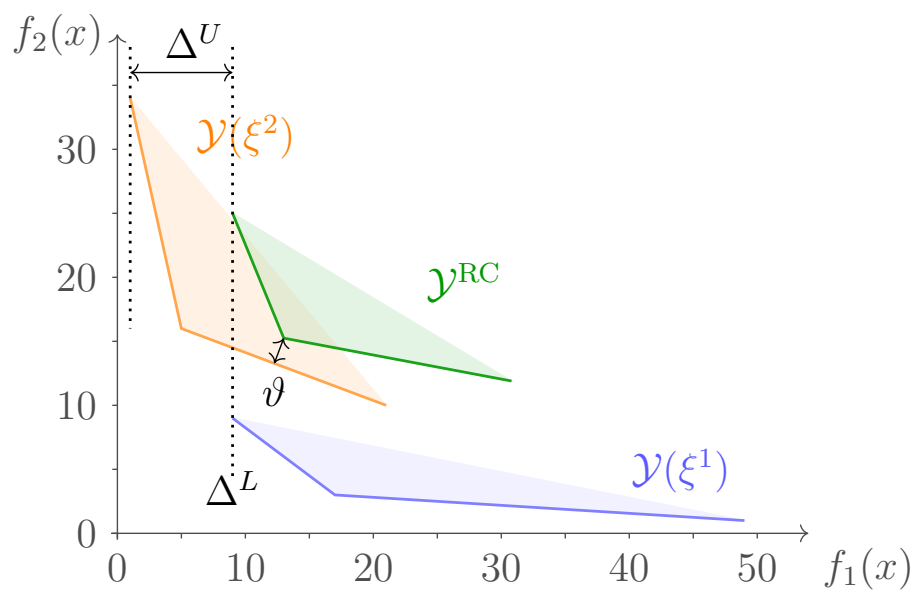

Figure 2: The lower robustness bound $\Delta^{L}$, the upper robustness bound $\Delta^{U}$ and the robustness gap $\vartheta$ for the uncertain MOP in Example 12.

Therefore,

$$
\Delta^{U}=\inf _{\xi \in \mathcal{U}} \max _{\substack{\lambda \in \mathbb{R}_{\geq}^{2},\|\lambda\|_{2}=1}} \Delta(\xi, \lambda)=\max _{\substack{\lambda \in \mathbb{R}_{\geq}^{2},\|\lambda\|_{2}=1}} \Delta\left(\xi^{2}, \lambda\right)=8 .
$$

The upper robustness bound $\Delta^{U}$ is also displayed in Figure 2.

The following proposition shows that $\Delta^{L}$ and $\Delta^{U}$ are well-defined, i.e., it is shown that the minima/maxima over all $\lambda \in \mathbb{R}_{\geq}^{p}$ with $\|\lambda\|_{*}=1$ and the minima over all $z \in \mathcal{Y}^{\mathrm{RC}}$ and all $y \in \mathcal{Y}(\xi), \xi \in \mathcal{U}$, exist.

Proposition 13. For every $\xi \in \mathcal{U}$ the map

$$
\Delta(\xi, \cdot):\left\{\lambda \in \mathbb{R}_{\geq}^{p} \mid\|\lambda\|_{*}=1\right\} \rightarrow \mathbb{R}, \quad \lambda \mapsto \Delta(\xi, \lambda)
$$

is continuous,

$$
\inf _{\lambda \in \mathbb{R}_{\geq}^{p},\|\lambda\|_{*}=1} \Delta(\xi, \lambda)=\min _{\substack{\lambda \in \mathbb{R}_{\geq}^{p} \\\|\lambda\|_{*}=1}} \Delta(\xi, \lambda)
$$

and

$$
\sup _{\lambda \in \mathbb{R}_{\geq}^{p},\|\lambda\|_{*}=1} \Delta(\xi, \lambda)=\max _{\substack{\lambda \in \mathbb{R}_{\geq}^{p} \\\|\lambda\|_{*}=1}} \Delta(\xi, \lambda) .
$$

I.e., the lower robustness bound $\Delta^{L}$ and the upper robustness bound $\Delta^{U}$ according to Definition 10 are well-defined.

Proof. Let $\xi \in \mathcal{U}$ be fixed. The set $\Lambda:=\left\{\lambda \in \mathbb{R}_{\geq}^{p} \mid\|\lambda\|_{*}=1\right\}$ is compact and, by assumption, the sets $\mathcal{Y}^{\mathrm{RC}}$ and $\mathcal{Y}(\xi)$ are compact. Hence, for $Y \in\left\{\mathcal{Y}^{\mathrm{RC}}, \mathcal{Y}(\xi)\right\}$, the inner 
product $\langle\cdot, \cdot\rangle: \Lambda \times Y,(\lambda, y) \mapsto \lambda^{T} y$ is uniformly continuous as a continuous function on the compact set $\Lambda \times Y$. For every $Y \in\left\{\mathcal{Y}^{\mathrm{RC}}, \mathcal{Y}(\xi)\right\}$, the auxiliary map

$$
h: \Lambda \rightarrow \mathbb{R}, \quad \lambda \mapsto \min _{y \in Y} \lambda^{T} y
$$

is continuous according to Berge's maximum theorem, see [Ber63, p. 116].

As a consequence, the function $\Delta(\xi, \cdot)$ is continuous, as a sum of two continuous functions, and it attains a minimum and a maximum over the compact set $\Lambda$.

In the two subsequent subsections we derive bounds for the multiobjective robustness gap.

\subsection{A lower bound}

We now show that $\Delta^{L}$ defined in Definition 10 is indeed a lower bound for the robustness gap $\vartheta$, i.e.,

$$
\Delta^{L} \leq \vartheta
$$

Theorem 14. The lower robustness bound $\Delta^{L}$ is a lower bound for the robustness gap $\vartheta$, i.e.,

$$
\inf _{\xi \in \mathcal{U}} \min _{\substack{\lambda \in \mathbb{R}_{\geq}^{p},\|\lambda\|_{*}=1}} \Delta(\xi, \lambda)=\Delta^{L} \leq \vartheta .
$$

Proof. Let $\bar{\xi} \in \mathcal{U}$, let $\bar{z} \in \mathcal{Y}_{P}^{\mathrm{RC}}$ and let $\bar{y} \in \mathcal{Y}_{P}(\bar{\xi})$. Due to the $\mathbb{R}_{\geq}^{p}$-convexity of $\mathcal{Y}(\bar{\xi})$ and the properties of the weighted-sum scalarization, there exists $\bar{\lambda} \in \mathbb{R}_{\geq}^{p},\|\bar{\lambda}\|_{*}=1$, such that $\bar{y} \in \mathcal{Y}_{P}(\bar{\xi})$ satisfies

$$
\bar{\lambda}^{T} \bar{y}=\min _{y \in \mathcal{Y}(\bar{\xi})} \bar{\lambda}^{T} y
$$

By Hölder's inequality, we know that

$$
\|\bar{z}-\bar{y}\|=\|\bar{\lambda}\|_{*}\|\bar{z}-\bar{y}\| \geq\left|\bar{\lambda}^{T}(\bar{z}-\bar{y})\right|
$$

and consequently

$$
\begin{aligned}
\|\bar{z}-\bar{y}\| & \geq \bar{\lambda}^{T} \bar{z}-\bar{\lambda}^{T} \bar{y} \\
& \geq \min _{z \in \mathcal{Y}^{\mathrm{RC}}} \bar{\lambda}^{T} z-\bar{\lambda}^{T} \bar{y} \\
& =\min _{z \in \mathcal{Y}^{\mathrm{RC}}} \bar{\lambda}^{T} z-\min _{y \in \mathcal{Y}(\bar{\xi})} \bar{\lambda}^{T} y \\
& =\Delta(\bar{\xi}, \bar{\lambda}) \\
& \geq \inf _{\xi \in \mathcal{U}} \min _{\substack{\lambda \in \mathbb{R}_{\geq}^{p} \\
\|\lambda\|_{*}=1}} \Delta(\xi, \lambda)=\Delta^{L},
\end{aligned}
$$

where the last equality results from Definition 10. Hence, $\Delta^{L} \leq\|z-y\|$ for all $z \in \mathcal{Y}_{P}^{\mathrm{RC}}$ and for all $y \in \mathcal{Y}_{P}(\xi)$ and $\xi \in \mathcal{U}$. Finally, we obtain

$$
\vartheta=\inf _{\xi \in \mathcal{U}} \inf _{z \in \mathcal{Y}_{P}^{\mathrm{RC}}} \inf _{y \in \mathcal{Y}_{P}(\xi)}\|z-y\| \geq \inf _{\xi \in \mathcal{U}} \inf _{z \in \mathcal{Y}_{P}^{\mathrm{RC}}} \inf _{y \in \mathcal{Y}_{P}(\xi)} \Delta^{L}=\Delta^{L} .
$$


If the robustness gap is zero, then $\Delta^{L}$ is a tight lower bound of $\vartheta$ as shown in Corollary 15.

Corollary 15. If $\vartheta=0$, then the lower robustness bound is a tight bound of the robustness gap, i.e., $\Delta^{L}=\vartheta$.

Proof. By Proposition 11, we have $0 \leq \Delta^{L}$. By Theorem 14, we obtain $0 \leq \Delta^{L} \leq \vartheta=0$.

\subsection{An upper bound}

In this section, we show that $\Delta^{U}$ defined in Definition 10 is indeed an upper bound on the robustness gap $\vartheta$ under some additional assumptions, i.e.,

$$
\vartheta \leq \Delta^{U}
$$

It is proven that this inequality holds under the following condition and it is shown that this condition is satisfied in many significant cases. These cases are distinguished by the norms on the primal and dual spaces. Two separate cases are proven that cover every choice of $\mathfrak{p}$-norms with $1 \leq \mathfrak{q} \leq \infty$ under at most one additional assumption. Moreover, it is shown that the condition is always satisfied for bicriteria problems, i.e, $\mathbb{R}^{p}=\mathbb{R}^{2}$, and every choice of $\mathfrak{p}$-norms with $1 \leq \mathfrak{q} \leq \infty$.

The condition depends on the scenario $\bar{\xi} \in \mathcal{U}$ and is the following.

Condition A. Let the primal space be $\left(\mathbb{R}^{p},\|\cdot\|\right)$ with the dual space $\left(\mathbb{R}^{p},\|\cdot\|_{*}\right)$. We say that Condition $A$ holds for a scenario $\bar{\xi} \in \mathcal{U}$ if there exist $\bar{z} \in \mathcal{Y}_{P}^{\mathrm{RC}}, \bar{y} \in \mathcal{Y}_{P}(\bar{\xi})$ and $\bar{\lambda} \in\left(\mathbb{R}^{p},\|\cdot\|_{*}\right)$ such that

(i) $\bar{\kappa}:=\bar{z}-\bar{y} \in \mathbb{R}_{\geq}^{p} \subseteq\left(\mathbb{R}^{p},\|\cdot\|\right)$ and $\bar{\lambda} \in \mathbb{R}_{\geq}^{p} \subseteq\left(\mathbb{R}^{p},\|\cdot\|_{*}\right)$

(ii) $\bar{\lambda} \in \arg \max _{\lambda \in \mathbb{R}_{\geq}^{p},\|\lambda\|_{*}=1} \bar{\kappa}^{T} \lambda$

(iii) $\bar{z} \in \arg \min _{z \in \mathcal{Y}^{\mathrm{RC}}} \bar{\lambda}^{T} z$.

In Figure 3, Condition A is illustrated for Example 12, the primal space $\left(\mathbb{R}^{2},\|\cdot\|_{1}\right)$ and the dual space $\left(\mathbb{R}^{2},\|\cdot\|_{\infty}\right)$. The following shows that $\Delta^{U}$ is an upper bound for the robustness gap if Condition A is satisfied.

Theorem 16. If Condition $A$ is satisfied for all $\xi \in \mathcal{U}$ with $\mathcal{Y}_{P}(\xi) \cap \mathcal{Y}_{P}^{\mathrm{RC}}=\emptyset$ then

$$
\vartheta \leq \Delta^{U}
$$

Proof. If there exists $\xi_{0} \in \mathcal{U}$ such that $\mathcal{Y}_{P}\left(\xi_{0}\right) \cap \mathcal{Y}_{P}^{\mathrm{RC}} \neq \emptyset$, then by Definition $8, \vartheta=\vartheta\left(\xi_{0}\right)=0$ and the claim follows immediately. Therefore, we assume that $\mathcal{Y}_{P}(\xi) \cap \mathcal{Y}_{P}^{\mathrm{RC}}=\emptyset$ for all $\xi \in \mathcal{U}$ and we consider any $\bar{\xi} \in \mathcal{U}$. By assumption, Condition A is satisfied for $\bar{\xi}$. Hence, there exist $\bar{z} \in \mathcal{Y}_{P}^{\mathrm{RC}}$ and $\bar{y} \in \mathcal{Y}_{P}(\bar{\xi})$ such that $\bar{\kappa}:=\bar{z}-\bar{y} \in \mathbb{R}_{\geq}^{p}$ and there exists $\bar{\lambda} \in \mathbb{R}_{\geq}^{p}$ such that

$$
\bar{\lambda} \in \underset{\lambda \in \mathbb{R}^{p},\|\lambda\|_{*}=1}{\arg \max } \bar{\kappa}^{T} \lambda \quad \text { and } \quad \bar{z} \in \underset{z \in \mathcal{Y}^{\mathrm{RC}}}{\arg \min } \bar{\lambda}^{T} z .
$$



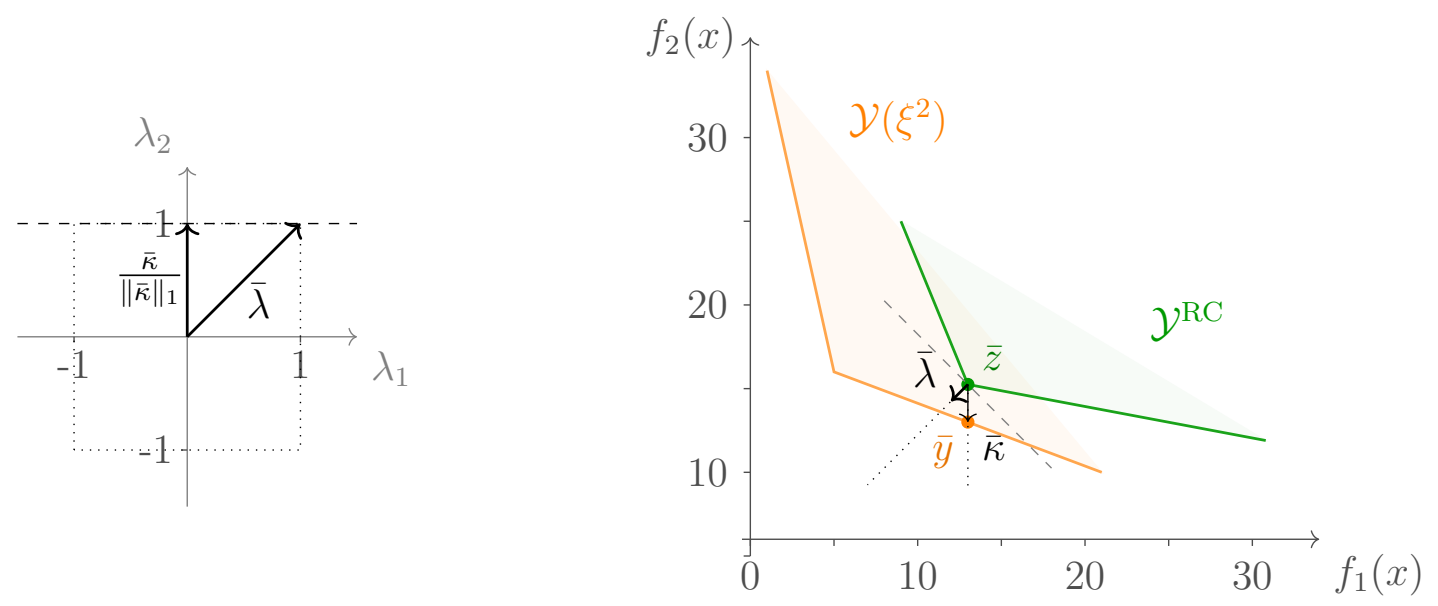

Figure 3: Illustration of Condition A. The left hand picture displays Condition A (i) and (ii), since $\bar{\lambda}$ is in the maximal level set of the linear function corresponding to the dashed line with the normal vector $\bar{\kappa}$. Condition A (iii) is depicted on the right hand side.

As a consequence, we obtain by Definition 10,

$$
\begin{aligned}
& \max _{\substack{\lambda \in \mathbb{R}_{\geq}^{p},\|\lambda\|_{*}=1}} \Delta(\bar{\xi}, \lambda) \quad \geq \Delta(\bar{\xi}, \bar{\lambda}) \\
& =\min _{z \in \mathcal{Y}^{\mathrm{RC}}} \bar{\lambda}^{T} z-\min _{y \in \mathcal{Y}(\bar{\xi})} \bar{\lambda}^{T} y=\bar{\lambda}^{T} \bar{z}-\min _{y \in \mathcal{Y}(\bar{\xi})} \bar{\lambda}^{T} y \\
& \geq \bar{\lambda}^{T} \bar{z}-\bar{\lambda}^{T} \bar{y} \quad=\bar{\lambda}^{T} \bar{\kappa},
\end{aligned}
$$

where the third equality follows from the right-hand side of (8) and the last equality follows from $\bar{\kappa}=\bar{z}-\bar{y}$. Using the left-hand side of (8) and the definition of the dual norm for $\|\bar{\kappa}\|$ and Definition 8, we have

$$
\begin{aligned}
\max _{\substack{\lambda \in \mathbb{R}_{\geq}^{p},\|\lambda\|_{*}=1}} \Delta(\bar{\xi}, \lambda) & \geq \bar{\lambda}^{T} \bar{\kappa} & =\max _{\substack{\lambda \in \mathbb{R}^{p},\|\lambda\|_{*}=1}} \bar{\kappa}^{T} \lambda \\
& =\|\bar{\kappa}\| & =\|\bar{z}-\bar{y}\| \\
& \geq \inf _{z \in \mathcal{Y}_{P}^{\mathrm{RC}}, y \in \mathcal{Y}_{P}(\bar{\xi})\|z-y\|} & =\vartheta(\bar{\xi})
\end{aligned}
$$

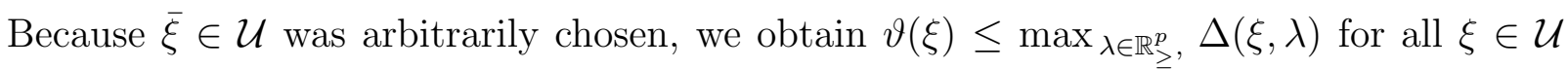
which directly implies $\|\lambda\|_{*}=1$

$$
\vartheta=\inf _{\xi \in \mathcal{U}} \vartheta(\xi) \leq \inf _{\xi \in \mathcal{U}} \max _{\lambda \in \mathbb{R}_{\geq}^{p},} \Delta(\xi, \lambda)=\Delta^{U}
$$

Remark 17. It can also be shown that if $\vartheta=\inf _{\xi \in \mathcal{U}} \vartheta(\xi)=\min _{\xi \in \mathcal{U}} \vartheta(\xi)$ and Condition $A$ is satisfied for some $\bar{\xi} \in \arg \min _{\xi \in \mathcal{U}} \vartheta(\xi)$, then there exists $\bar{\lambda} \in \mathbb{R}_{\geq}^{p} \subseteq\left(\mathbb{R}^{p},\|\cdot\|_{*}\right)$ with $\|\bar{\lambda}\|_{*}=1$ such that

$$
\vartheta=\Delta(\bar{\xi}, \bar{\lambda}) .
$$


If Condition $\mathrm{A}$ is satisfied for a scenario $\xi \in \mathcal{U}$, it may not hold for every $\bar{z} \in \mathcal{Y}_{P}^{\mathrm{RC}}$ and $\bar{y} \in \mathcal{Y}_{P}(\xi)$. It can be checked in Figure 3 that for the rightmost point $z=(29.81,10.91) \in$ $\mathcal{Y}_{P}^{\mathrm{RC}}$, there do not exist any $y \in \mathcal{Y}_{P}\left(\xi^{2}\right)$ and $\lambda \geq 0$ that satisfy Condition $\mathrm{A}$.

In the following, we investigate three cases in which Condition $\mathrm{A}$ is satisfied. First, it is shown that Condition A holds if the primal norm is the $\mathfrak{p}$-norm with $\mathfrak{p}=1$ (Lemma 18). Second, it is proven that Condition A is satisfied if the primal norm is any $\mathfrak{p}$-norm with $1 \leq \mathfrak{p} \leq \infty$ under an additional assumption for the ideal point of RC (Lemma 19). Third, it is shown that Condition A always holds for biobjective problems without further assumptions whenever the primal norm is any $\mathfrak{p}$-norm with $1 \leq \mathfrak{p} \leq \infty$ (Lemma 20).

We first show that Condition A holds for all scenarios $\bar{\xi}$ with $\mathcal{Y}_{P}(\bar{\xi}) \cap \mathcal{Y}_{P}^{\mathrm{RC}}=\emptyset$ if the primal norm is the $\mathfrak{p}$-norm with $\mathfrak{p}=1$.

Lemma 18. Let the primal norm be $\|\cdot\|=\|\cdot\|_{1}$ with the dual norm $\|\cdot\|_{*}=\|\cdot\|_{\infty}$. If $\mathcal{Y}_{P}(\bar{\xi}) \cap \mathcal{Y}_{P}^{\mathrm{RC}}=\emptyset$ for a scenario $\bar{\xi} \in \mathcal{U}$, then Condition $A$ is satisfied for $\bar{\xi}$.

Proof. Let $\mathcal{Y}_{P}(\bar{\xi}) \cap \mathcal{Y}_{P}^{\mathrm{RC}}=\emptyset$ and set $\bar{\lambda}:=(1,1, \ldots, 1)^{T} \in \mathbb{R}_{\geq}^{p}$. Because $\mathcal{Y}^{\mathrm{RC}}$ is compact, there exists

$$
\bar{z} \in \underset{z \in \mathcal{Y}^{\mathrm{RC}}}{\arg \min } \bar{\lambda}^{T} z
$$

hence, $\bar{z} \in \mathcal{Y}_{P}^{\mathrm{RC}}$ and by Corollary 5 , there exists $\bar{y} \in \mathcal{Y}_{P}(\bar{\xi})$ such that $\bar{y} \in\{\bar{z}\}-\mathbb{R}_{\geqq}^{p}$. By assumption, $\mathcal{Y}_{P}(\bar{\xi}) \cap \mathcal{Y}_{P}^{\mathrm{RC}}=\emptyset$, hence $\bar{z} \neq \bar{y}$ and $\bar{y} \in\{\bar{z}\}-\mathbb{R}_{\geq}^{p}$. We set $\bar{\kappa}:=\bar{z}-\bar{y} \in \mathbb{R}_{\geq}^{p}$. Then Condition A (i) and (iii) are satisfied. Furthermore, the dual norm to $\|\cdot\|=\|\cdot\|_{1}$ is $\|\cdot\|_{*}=\|\cdot\|_{\infty}$, and we obtain

$$
\max _{\substack{\lambda \in \mathbb{R}^{p},\|\lambda\|_{\infty}=1}} \bar{\kappa}^{T} \lambda=\max _{\substack{\lambda \in \mathbb{R}^{p},\|\lambda\|_{\infty}=1}} \sum_{i=1}^{p} \underbrace{\bar{\kappa}_{i}}_{\geq 0} \underbrace{\lambda_{i}}_{\leq 1} \leq \sum_{i=1}^{p} \bar{\kappa}_{i}=\bar{\kappa}^{T} \bar{\lambda}
$$

and therefore $\bar{\lambda} \in \arg \max _{\lambda \geq 0,\|\lambda\|_{\infty}=1} \bar{\kappa}^{T} \lambda$, which proves Condition A (ii).

Moreover, Condition A is satisfied if the primal norm on $\left(\mathbb{R}^{p},\|\cdot\|\right)$ is a $\mathfrak{p}$-norm $\|\cdot\|_{\mathfrak{p}}$ with $\mathfrak{p} \in(1, \infty]$ if an additional assumption holds.

Lemma 19. Let the primal norm be $\|\cdot\|=\|\cdot\|_{\mathfrak{p}}$ for $\mathfrak{p} \in(1, \infty]$ with the dual norm $\|\cdot\|_{*}=\|\cdot\|_{\mathfrak{q}}$ where $\mathfrak{q} \in[1, \infty)$ and $\frac{1}{\mathfrak{p}}+\frac{1}{\mathfrak{q}}=1$. If $\mathcal{Y}_{P}(\bar{\xi}) \cap \mathcal{Y}_{P}^{\mathrm{RC}}=\emptyset$ for a scenario $\bar{\xi} \in \mathcal{U}$ and if the ideal point of RC satisfies $z_{\mathrm{RC}}^{I} \in \mathcal{Y}(\bar{\xi})+\mathbb{R}_{\geqq}^{p}$, then Condition $A$ is satisfied for $\bar{\xi}$.

Proof. By assumption, $z_{\mathrm{RC}}^{I} \in \mathcal{Y}(\bar{\xi})+\mathbb{R}_{\geqq}^{p}$ for the scenario $\bar{\xi} \in \mathcal{U}$. Then there exists $y^{\prime} \in \mathcal{Y}(\bar{\xi})$ such that $y^{\prime} \in\left\{z_{\mathrm{RC}}^{I}\right\}-\mathbb{R}_{\geqq}^{p}$ and there exists $\bar{y} \in \mathcal{Y}_{P}(\bar{\xi})$ such that $\bar{y} \in\left\{y^{\prime}\right\}-\mathbb{R}_{\geqq}^{p} \subseteq\left\{z_{\mathrm{RC}}^{I}\right\}-\mathbb{R}_{\geqq}^{p}$ according to [Luc84, Prop. 3.1] which is applicable because $\mathcal{Y}(\bar{\xi})$ is compact.

Furthermore, there exists $\bar{z} \in\left(\arg \min _{z \in \mathcal{Y}^{\mathrm{RC}}}\|z-\bar{y}\|_{\mathfrak{p}}\right) \cap \mathcal{Y}_{P}^{\mathrm{RC}}$, which follows from [Ehr05, Prop. 4.21 (2.)] for $\mathfrak{p} \in(1, \infty)$, and from [Ehr05, Prop. $4.22(2$.$) ] for \mathfrak{p}=\infty$ since $\bar{y} \leqq z_{\mathrm{RC}}^{I}$ 
and $\mathcal{Y}^{\mathrm{RC}}$ is compact. Consequently, $\bar{y} \leqq z_{\mathrm{RC}}^{I} \leqq \bar{z}$ and the assumption $\mathcal{Y}_{P}^{\mathrm{RC}} \cap \mathcal{Y}_{P}(\bar{\xi})=\emptyset$ implies $\bar{z} \neq \bar{y}$, which results in $\bar{\kappa}:=\bar{z}-\bar{y} \in \mathbb{R}_{\geq}^{p}$.

Because the sets $B_{\mathfrak{p}}\left(\bar{y},\|\bar{z}-\bar{y}\|_{\mathfrak{p}}\right)$ and $\mathcal{Y}^{\mathrm{RC}}+\mathbb{R}_{\geqq}^{p}$ are convex, the Eidelheit separation theorem can be applied, see, e.g., [Lue69]. That is, there exists a vector in the dual space, $\bar{\lambda} \in$ $\left(\mathbb{R}^{p},\|\cdot\|_{\mathfrak{q}}\right)$ with $\|\bar{\lambda}\|_{\mathfrak{q}}=1$, such that for all $y \in B_{\mathfrak{p}}\left(\bar{y},\|\bar{z}-\bar{y}\|_{\mathfrak{p}}\right)$ and for all $z \in \mathcal{Y}^{\mathrm{RC}}$

$$
\bar{\lambda}^{T} y \leq \bar{\lambda}^{T} \bar{z} \leq \bar{\lambda}^{T} z .
$$

Hence,

$$
\bar{z} \in \underset{y \in B_{\mathfrak{p}}\left(\bar{y},\|\bar{z}-\bar{y}\|_{\mathfrak{p}}\right)}{\arg \max } \bar{\lambda}^{T} y \quad \text { and } \quad \bar{z} \in \underset{z \in \mathcal{Y}^{\mathrm{RC}}}{\arg \min } \bar{\lambda}^{T} z
$$

where the left-hand side leads to

$$
\bar{\kappa} \in \underset{\lambda \in B_{\mathfrak{p}}\left(0,\|\bar{\kappa}\|_{\mathfrak{p}}\right)}{\arg \max } \bar{\lambda}^{T} \lambda
$$

In order to show $\bar{\lambda} \in \mathbb{R}_{\geq}^{p}$, we distinguish the two cases $\mathfrak{p}=\infty$ and $\mathfrak{p} \in(1, \infty)$. First, let $\mathfrak{p}=\infty$ and we assume that there is an index $1 \leq j \leq p$ such that $\bar{\lambda}_{j}<0$. Because $\bar{y} \leq \bar{z}$, there exists $\varepsilon>0$ such that $\bar{y}_{j}-1<\bar{z}_{j}-\varepsilon<\bar{z}_{j}$ and, hence, $\bar{z}-\varepsilon \cdot e_{j} \in B_{\infty}\left(\bar{y},\|\bar{z}-\bar{y}\|_{\infty}\right)$, where $e_{j} \in \mathbb{R}^{p}$ is the $j$-th unit vector. Then $\bar{\lambda}^{T}\left(\bar{z}-\varepsilon \cdot e_{j}\right)=\bar{\lambda}^{T} \bar{z}-\varepsilon \bar{\lambda}_{j}>\bar{\lambda}^{T} \bar{z}$ in contradiction to the left-hand side of (9).

Second, let $\mathfrak{p} \in(1, \infty)$ and we assume that there is an index $1 \leq j \leq p$ such that $\bar{\lambda}_{j}<0$. Due to the duality of the norms $\|\cdot\|_{\mathfrak{p}}$ and $\|\cdot\|_{\mathfrak{q}}$ and (10), we obtain $\|\bar{\lambda}\|_{\mathfrak{q}}=\max _{\|\lambda\|_{\mathfrak{p}}=1} \bar{\lambda}^{T} \lambda=$ $\bar{\lambda}^{T} \frac{\bar{\kappa}}{\|\bar{\kappa}\|_{\mathfrak{p}}}$ and hence $\bar{\lambda}^{T} \bar{\kappa}=\|\bar{\kappa}\|_{\mathfrak{p}}\|\bar{\lambda}\|_{\mathfrak{q}}$. Consequently, Hölder's inequality is satisfied by $\bar{\kappa}$ and $\bar{\lambda}$ with equality and by the conditions for Hölder's inequality being satisfied with equality for $\mathfrak{p} \in(1, \infty)$, see, e.g., [Cve12, Th. 9.2], we obtain that there exists $\alpha \in \mathbb{R} \backslash\{0\}$ such that $\bar{\kappa}_{j}^{\mathfrak{p}}=\alpha \cdot \bar{\lambda}_{j}^{\mathfrak{q}} \neq 0$. Then $\bar{\kappa}_{j}>0, \kappa^{\prime}:=\bar{\kappa}-2 \bar{\kappa}_{j} e_{j} \in B_{\mathfrak{p}}\left(0,\|\bar{\kappa}\|_{\mathfrak{p}}\right)$, where $e_{j} \in \mathbb{R}^{p}$ is the $j$-th unit vector, and $\bar{\lambda}^{T} \kappa^{\prime}=\bar{\lambda}^{T} \bar{\kappa}-2 \bar{\lambda}_{j} \bar{\kappa}_{j}>\bar{\lambda}^{T} \bar{\kappa}$ in contradiction to (10).

As a consequence of both cases, we have $\bar{\lambda} \in \mathbb{R}_{\geq}^{p}$ for both $\mathfrak{p}=\infty$ and $\mathfrak{p} \in(1, \infty)$ and Condition A (i) and (iii) are satisfied. It remains to show Condition A (ii), i.e., $\bar{\lambda} \in$ $\arg \max _{\lambda \in \mathbb{R}^{p},\|\lambda\|_{\mathfrak{q}}=1} \bar{\kappa}^{T} \lambda$. Let $\lambda_{\diamond} \in \arg \max _{\lambda \in \mathbb{R}^{p},\|\lambda\|_{\mathfrak{q}}=1} \bar{\kappa}^{T} \lambda$, then, because $\|\cdot\|_{\mathfrak{q}}$ is the dual norm of $\|\cdot\|_{\mathfrak{p}}$, and by (10),

$$
1=\|\bar{\lambda}\|_{\mathfrak{q}}=\max _{\substack{\lambda \in \mathbb{R}^{p},\|\lambda\|_{\mathfrak{p}}=1}} \bar{\lambda}^{T} \lambda=\bar{\lambda}^{T} \frac{\bar{\kappa}}{\|\bar{\kappa}\|_{\mathfrak{p}}} \leq \max _{\substack{\lambda \in \mathbb{R}^{p},\|\lambda\|_{\mathfrak{q}}=1}} \frac{\bar{\kappa}^{T} \lambda}{\|\bar{\kappa}\|_{\mathfrak{p}}}=\frac{\bar{\kappa}^{T} \lambda_{\diamond}}{\|\bar{\kappa}\|_{\mathfrak{p}}} \leq \frac{\|\bar{\kappa}\|_{\mathfrak{p}}}{\|\bar{\kappa}\|_{\mathfrak{p}}}\left\|\lambda_{\diamond}\right\|_{\mathfrak{q}}=1
$$

where the second inequality follows from Hölder's inequality. Consequently, all inequalities are equalities and, because the first inequality is an equality, $\bar{\lambda} \in \arg \max _{\lambda \in \mathbb{R}^{p},\|\lambda\|_{\mathfrak{q}}=1} \bar{\kappa}^{T} \lambda$.

Lemma 19 is instrumental to establish Condition A and the result of Theorem 16 and requires the special assumption about the location of the ideal point of $\mathrm{RC}, z_{\mathrm{RC}}^{I}$, with respect to the outcome set $\mathcal{Y}(\bar{\xi})$. In particular, it is shown that $\bar{y}$ serves as a utopia point for the minimum norm problem solved on $\mathcal{Y}^{\mathrm{RC}}$ while $\bar{z} \in \mathcal{Y}_{P}^{\mathrm{RC}}$ is an optimal solution to 
this problem. Since $\bar{y}$ is a utopia point, we also have $\bar{y} \leq \bar{z}$ and we immediately obtain $\bar{\kappa} \in \mathbb{R}_{\geq}^{p}$.

Lemma 20 corresponds to a biobjective version of Lemma 18 and Lemma 19 in which the additional assumption on the ideal point is dropped. In absence of this assumption, the location of the point $\bar{y} \in \mathcal{Y}_{P}(\bar{\xi})$ with respect to $\mathcal{Y}^{\mathrm{RC}}$ is unknown and therefore this point cannot play the role of a utopia point as it does in the multiobjective case. The existence of the direction vector $\bar{\kappa}$ is thus supported by solving the minimum norm problem with the ideal point $y_{\mathrm{P}(\bar{\xi})}^{I}$ of $\mathcal{Y}(\bar{\xi})$ as a utopia point of $\mathcal{Y}^{\mathrm{RC}}$ and the fact that $p=2$.

Lemma 20. Let $\mathbb{R}^{p}=\mathbb{R}^{2}$ and let the primal norm be $\|\cdot\|=\|\cdot\|_{\mathfrak{p}}$ for $\mathfrak{p} \in[1, \infty]$ with the dual norm $\|\cdot\|_{*}=\|\cdot\|_{\mathfrak{q}}$ where $\mathfrak{q} \in[1, \infty]$ and $\frac{1}{\mathfrak{p}}+\frac{1}{\mathfrak{q}}=1$. If $\mathcal{Y}_{P}(\bar{\xi}) \cap \mathcal{Y}_{P}^{\mathrm{RC}}=\emptyset$ for a scenario $\bar{\xi} \in \mathcal{U}$, then Condition $A$ is satisfied for $\bar{\xi}$.

Proof. Let $z_{\mathrm{RC}}^{I}$ denote the ideal point of RC and let $y_{\mathrm{P}(\bar{\xi})}^{I}$ denote the ideal point of $\mathrm{P}(\bar{\xi})$. Note that, if $z_{\mathrm{RC}}^{I} \in \mathcal{Y}(\bar{\xi})+\mathbb{R}_{\geq}^{2}$, then the claim follows directly from Lemma 19 .

Hence, in the following, we only consider the case $z_{\mathrm{RC}}^{I} \notin \mathcal{Y}(\bar{\xi})+\mathbb{R}_{\geqq}^{2}$, which also implies $y_{\mathrm{P}(\bar{\xi})}^{I} \notin \mathcal{Y}(\bar{\xi})+\mathbb{R}_{\geqq}^{2}$ because otherwise $z_{\mathrm{RC}}^{I} \in\left\{y_{\mathrm{P}(\bar{\xi})}^{I}\right\}+\mathbb{R}_{\geqq}^{2} \subseteq \mathcal{Y}(\bar{\xi})+\mathbb{R}_{\geqq}^{2}$. We show that there exist $\bar{y} \in \mathcal{Y}_{P}(\bar{\xi})$ and $\bar{z} \in\left(\arg \min _{z \in \mathcal{Y}^{\mathrm{RC}}}\|z-\bar{y}\|_{\mathfrak{p}}\right) \cap \mathcal{Y}_{P}^{\mathrm{RC}}$ such that $\bar{\kappa}:=\bar{z}-\bar{y} \in \mathbb{R}_{\geq}^{2}$.

By Proposition 4, we have $\mathcal{Y}^{\mathrm{RC}} \subseteq \mathcal{Y}(\bar{\xi})+\mathbb{R}_{\geqq}^{2}$ and hence $y_{\mathrm{P}(\bar{\xi})}^{I} \leqq z_{\mathrm{RC}}^{I}$. Then $y_{\mathrm{P}(\bar{\xi})}^{I}$ is the ideal point or a utopia point for $\mathrm{RC}$ and there exists

$$
\bar{z} \in\left(\underset{z \in \mathcal{Y}^{\mathrm{RC}}}{\arg \min }\left\|z-y_{\mathrm{P}(\bar{\xi})}^{I}\right\|_{\mathfrak{p}}\right) \cap \mathcal{Y}_{P}^{\mathrm{RC}},
$$

which follows from [Ehr05, Prop. 4.21 (2.)] for $\mathfrak{p} \in(1, \infty)$, and from [Ehr05, Prop. 4.22 (2.)] for $\mathfrak{p}=\infty$ since $\mathcal{Y}^{\mathrm{RC}}$ is compact.

Because $\bar{z} \in \mathcal{Y}(\bar{\xi})+\mathbb{R}_{\geqq}^{2}, y_{\mathrm{P}(\bar{\xi})}^{I} \notin \mathcal{Y}(\bar{\xi})+\mathbb{R}_{\geqq}^{2}$, and $\mathcal{Y}(\bar{\xi})+\mathbb{R}_{\geqq}^{2}$ is convex and closed, the intersection of bd $\left(\mathcal{Y}(\bar{\xi})+\mathbb{R}_{\geqq}^{2}\right)$ with the line segment between $y_{\mathrm{P}(\bar{\xi})}^{I}$ and $\bar{z}$ is nonempty and compact and

$$
\bar{y}:=\arg \min \left\{\left\|y-y_{\mathrm{P}(\bar{\xi})}^{I}\right\|_{\mathfrak{p}} \mid y \in \operatorname{conv}\left(\left\{y_{\mathrm{P}(\bar{\xi})}^{I}, \bar{z}\right\}\right) \cap \operatorname{bd}\left(\mathcal{Y}(\bar{\xi})+\mathbb{R}_{\geqq}^{2}\right)\right\}
$$

is well-defined because the minimum is attained at exactly one point. Hence, $y_{\mathrm{P}(\bar{\xi})}^{I} \leq \bar{y} \leqq \bar{z}$. Because of the assumption $\mathcal{Y}_{P}(\bar{\xi}) \cap \mathcal{Y}_{P}^{\mathrm{RC}}=\emptyset$, we have $\bar{y} \neq \bar{z}$, and so $\bar{\kappa}:=\bar{z}-\bar{y} \in \mathbb{R}_{\geq}^{2}$. We obtain $\bar{z} \in \arg \min _{z \in \mathcal{Y}^{\mathrm{RC}}}\|z-\bar{y}\|_{\mathfrak{p}}$ because

$$
\begin{aligned}
\left\|\bar{z}-y_{\mathrm{P}(\bar{\xi})}^{I}\right\|_{\mathfrak{p}} & =\min _{z \in \mathcal{Y}^{\mathrm{RC}}}\left\|z-y_{\mathrm{P}(\bar{\xi})}^{I}\right\|_{\mathfrak{p}} \leq\left\|\bar{y}-y_{\mathrm{P}(\bar{\xi})}^{I}\right\|_{\mathfrak{p}}+\min _{z \in \mathcal{Y}^{\mathrm{RC}}}\|z-\bar{y}\|_{\mathfrak{p}} \\
& \leq\left\|\bar{y}-y_{\mathrm{P}(\bar{\xi})}^{I}\right\|_{\mathfrak{p}}+\|\bar{z}-\bar{y}\|_{\mathfrak{p}}=\left\|\bar{z}-y_{\mathrm{P}(\bar{\xi})}^{I}\right\|_{\mathfrak{p}},
\end{aligned}
$$

i.e., all inequalities have to be equalities. It remains to show that $\bar{y} \in \mathcal{Y}_{P}(\bar{\xi})$. Note that there exist $y^{1} \in \arg \min _{y \in \mathcal{Y}_{P}(\bar{\xi})}(1,0) y$ and $y^{2} \in \arg \min _{y \in \mathcal{Y}_{P}(\bar{\xi})}(0,1) y$ according to [Luc84, 
Prop. 3.1] since $\mathcal{Y}(\bar{\xi})$ is compact and, because the objective space is two-dimensional, we have

$$
\operatorname{bd}\left(\mathcal{Y}(\bar{\xi})+\mathbb{R}_{\geqq}^{2}\right)=\mathcal{Y}_{P}(\bar{\xi}) \cup\left\{y^{1}+r \cdot(0,1)^{T} \mid r \in \mathbb{R}_{>}\right\} \cup\left\{y^{2}+r \cdot(1,0)^{T} \mid r \in \mathbb{R}_{>}\right\} .
$$

For a contradiction, assume that $\bar{y} \in \mathrm{bd}\left(\mathcal{Y}(\bar{\xi})+\mathbb{R}_{\geqq}^{2}\right) \backslash \mathcal{Y}_{P}(\bar{\xi})$. By (12), we obtain $\bar{y} \in$ $\left\{y^{1}+r \cdot(0,1)^{T} \mid r \in \mathbb{R}_{>}\right\} \cup\left\{y^{2}+r \cdot(1,0)^{T} \mid r \in \mathbb{R}_{>}\right\}$and we assume without loss of generality that $\bar{y} \in\left\{y^{2}+r \cdot(1,0)^{T} \mid r \in \mathbb{R}_{>}\right\}$. Note that $\bar{y}_{2}=y_{2}^{2}=y_{\mathrm{P}(\bar{\xi}) 2}^{I}$ and by $(11)$, we have $\bar{z}_{2}=\bar{y}_{2}=y_{\mathrm{P}(\bar{\xi}) 2}^{I}$. Because we assumed $\bar{y} \notin \mathcal{Y}_{P}(\bar{\xi})$, we have $y_{1}^{2}<\bar{y}_{1}$. Therefore, $y^{2}$ is an element of the line segment between $y_{\mathrm{P}(\bar{\xi})}^{I}$ and $\bar{z}$, and we have $\left|y_{2}^{2}-y_{\mathrm{P}(\bar{\xi}) 2}^{I}\right|=0$ and $\left|\bar{y}_{1}-y_{\mathrm{P}(\bar{\xi}) 1}^{I}\right|>\left|y_{1}^{2}-y_{\mathrm{P}(\bar{\xi}) 1}^{I}\right|$ in contradiction to $\bar{y}$ being the point closest to $y_{\mathrm{P}(\bar{\xi})}^{I}$ by definition. Therefore, $\bar{y} \in \mathcal{Y}_{P}(\bar{\xi})$.

In summary, there exist $\bar{y} \in \mathcal{Y}_{P}(\bar{\xi})$ and $\bar{z} \in\left(\arg \min _{z \in \mathcal{Y}^{\mathrm{RC}}}\|z-\bar{y}\|_{\mathfrak{p}}\right) \cap \mathcal{Y}_{P}^{\mathrm{RC}}$ such that $\bar{\kappa}:=\bar{z}-\bar{y} \in \mathbb{R}_{\geq}^{2}$. The remainder of the proof is analogous to the proof of Lemma 19 .

For the Euclidean norm $\|\cdot\|=\|\cdot\|_{2}=\|\cdot\|_{*}$, the primal and dual space coincide and so do the vectors $\bar{\lambda}$ and $\bar{\kappa}$ in Condition $\mathrm{A}$. In this case, we relate Condition A to normal cones as defined in [Roc15].

Remark 21. Using the normal cone to $\mathcal{Y}^{\mathrm{RC}}$ at $\bar{z} \in \mathcal{Y}^{\mathrm{RC}}$ as defined in [Roc15], i.e., $\mathcal{N}_{\mathcal{Y}} \mathrm{RC}(\bar{z}):=\left\{v \in \mathbb{R}^{p} \mid\langle v, \bar{z}-z\rangle \leq 0 \forall z \in \mathcal{Y}^{\mathrm{RC}}\right\}$, we obtain the following. Condition $A$ is satisfied for a scenario $\bar{\xi} \in \mathcal{U}$ if $\mathcal{Y}_{P}(\bar{\xi}) \cap \mathcal{Y}_{P}^{\mathrm{RC}}=\emptyset$ and there exists $\bar{z} \in \mathcal{Y}^{\mathrm{RC}}$ such that

$$
\left(\{\bar{z}\}+\left(\mathcal{N}_{\mathcal{Y}^{\mathrm{RC}}}(\bar{z}) \cap\left(-\mathbb{R}_{\geq}^{p}\right)\right)\right) \cap \mathcal{Y}_{P}(\bar{\xi}) \neq \emptyset .
$$

A further sufficient condition for Condition A to be satisfied can be formulated via restricted normal cones as defined in [BLPW13].

\subsection{A condition for the zero robustness gap}

The main result about the robustness gap in the original papers on single-objective robust optimization, see [BTN98, Th. 2.1] and [BTN99, Prop. 2.1], is that the robustness gap is zero if the uncertainty is constraint-wise, i.e., if the uncertainty set is a Cartesian product of the uncertainty sets of the constraints. In this section, we extend that result and we provide a condition on the uncertainty set $\mathcal{U}$ that has two effects. Under the condition, for each point in the robust Pareto set $z \in \mathcal{Y}_{P}^{\mathrm{RC}}$ there is a scenario $\xi \in \mathcal{U}$ whose Pareto set $\mathcal{Y}_{P}(\xi)$ is arbitrarily close to $z$. Also, the condition is sufficient for $\vartheta=0$.

In the following the uncertainty is required to be constraint-wise and objective-wise as also studied in [KL12, EIS14]. The uncertainty set $\mathcal{U}$ in $\{\mathrm{P}(\xi)\}_{\xi \in \mathcal{U}}$ is called constraint-wise and objective-wise if $\mathcal{U}=\mathcal{U}_{1} \times \cdots \times \mathcal{U}_{p} \times \mathcal{U}_{p+1} \times \cdots \times \mathcal{U}_{p+m}$ such that $\{\mathrm{P}(\xi)\}_{\xi \in \mathcal{U}}$ can equivalently be written as 


$$
\left\{\begin{array}{rrr}
(\mathrm{P}(\xi)) & \min _{x}\left(f_{1}\left(x, \xi_{1}\right), \ldots, f_{p}\left(x, \xi_{p}\right)\right)^{T} \\
\text { s.t. } & F_{j}\left(x, \xi_{p+j}\right) & \geqq 0 \quad \forall j=1, \ldots, m \\
x & \in \mathbb{R}^{n}
\end{array}\right\} .
$$

The following assumption corresponds to the setting for the single-objective robustness gap result in [BTN98] plus the assumption of objective-wise uncertainty. Recall that, by the Convexity Assumption at the beginning of Section 3, $\mathrm{P}(\xi)$ is assumed to be a convex problem for every $\xi \in \mathcal{U}$.

\section{Assumption 1.}

(i) There exists a compact convex set $\mathfrak{X} \subseteq \mathbb{R}^{n}$ with int $(\mathfrak{X}) \neq \emptyset$ such that $\bigcup_{\xi \in \mathcal{U}} X(\xi) \subseteq \mathfrak{X}$.

(ii) The uncertainty set $\mathcal{U}$ is closed and convex and $\operatorname{int}(\mathcal{U}) \neq \emptyset$.

(iii) For every $x \in \mathfrak{X}$, the functions $f(x, \cdot): \mathcal{U} \rightarrow \mathbb{R}^{p}$ and $F(x, \cdot): \mathcal{U} \rightarrow \mathbb{R}^{m}$ are affine in $\xi$.

(iv) $\mathcal{U}$ is constraint-wise and objective-wise.

Under Assumption 1, every point in the robust Pareto set is arbitrarily close to the set of Pareto sets of the scenarios as the following result shows.

Proposition 22. Let the Continuity Assumption, the Convexity Assumption, and Assumption 1 hold.

Then for every $z^{*} \in \mathcal{Y}_{P}^{\mathrm{RC}}$ and every $\varepsilon>0$, there exists a scenario $\bar{\xi} \in \mathcal{U}$ such that

$$
\operatorname{dist}\left(\left\{z^{*}\right\}, \mathcal{Y}_{P}(\bar{\xi})\right)<\varepsilon
$$

and

$$
\inf _{\xi \in \mathcal{U}} \inf _{y \in \mathcal{Y}_{P}(\xi)}\left\|z^{*}-y\right\|=0 .
$$

Proof. Let $z^{*} \in \mathcal{Y}_{P}^{\mathrm{RC}}$ and let $\varepsilon>0$. Because of the Continuity Assumption, the Convexity Assumption, and Assumption 1 (i), [Har78, Th. 5.5] and [Geo68, Th. 1] can be applied and hence, there exist $\bar{z} \in \mathcal{Y}_{P}^{\mathrm{RC}}$ and a vector $\bar{\lambda}$ in the dual space, $\bar{\lambda} \in \mathbb{R}_{>}^{p}$ with $\|\bar{\lambda}\|_{*}=1$, such that

$$
\left\|z^{*}-\bar{z}\right\|<\frac{\varepsilon}{2} \quad \text { and } \quad \bar{z} \in \underset{z \in \mathcal{Y}^{\mathrm{RC}}}{\arg \min } \bar{\lambda}^{T} z
$$

We next show that there exists a scenario $\bar{\xi} \in \mathcal{U}$ such that $\operatorname{dist}\left(\{\bar{z}\}, \mathcal{Y}_{P}(\bar{\xi})\right)<\frac{\varepsilon}{2}$.

The map $\lambda^{T} f(x, \cdot): \mathcal{U} \rightarrow \mathbb{R}$ is affine in $\xi$ for all $x \in \mathbb{R}^{n}$ and all $\lambda \in \mathbb{R}^{p}$ as a composition of a linear and an affine function, and the map $\lambda^{T} f(\cdot, \xi): \mathbb{R}^{n} \rightarrow \mathbb{R}$ is convex in $x$ for all $\xi \in \mathcal{U}$ and all $\lambda \in \mathbb{R}_{\geqq}^{p}$ as a nonnegative sum of convex functions. Therefore, the uncertain single-objective optimization problem

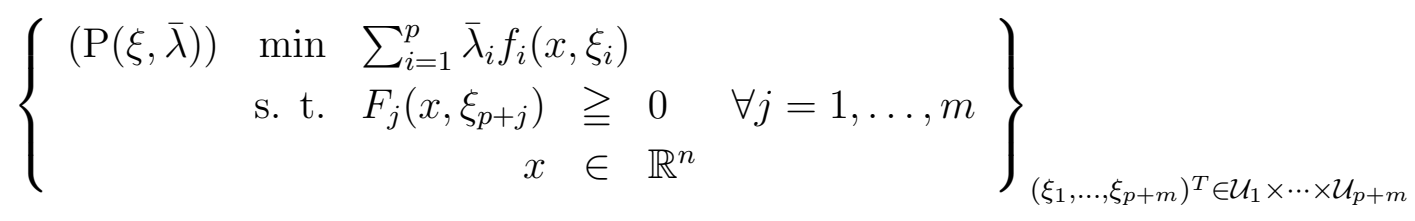


satisfies Assumption 1 (i) - (iii) as required in [BTN98, Th. 2.1], which we apply to Definition 6, and we obtain

$$
\vartheta^{s-o}:=\min _{x \in X^{\mathrm{RC}}} \sup _{\xi \in \mathcal{U}} \bar{\lambda}^{T} f(x, \xi)-\sup _{\xi \in \mathcal{U}} \min _{x \in X(\xi)} \bar{\lambda}^{T} f(x, \xi)=0 .
$$

Because the uncertainty is objective-wise, we have for all $x \in \mathbb{R}^{n}$

$$
\begin{aligned}
\sup _{\xi \in \mathcal{U}} \bar{\lambda}^{T} f(x, \xi) & =\sup _{\left(\xi_{1}, \ldots, \xi_{p}\right) \in \mathcal{U}_{1} \times \cdots \times \mathcal{U}_{p}} \sum_{i=1}^{p} \bar{\lambda}_{i} f_{i}\left(x, \xi_{i}\right) \\
=\sum_{i=1}^{p} \bar{\lambda}_{i} \sup _{\xi_{i} \in \mathcal{U}_{i}} f_{i}\left(x, \xi_{i}\right) & =\bar{\lambda}^{T} f^{\mathrm{RC}}(x)
\end{aligned}
$$

and hence,

$$
\min _{x \in X^{\mathrm{RC}}} \bar{\lambda}^{T} f^{\mathrm{RC}}(x)-\sup _{\xi \in \mathcal{U}} \min _{x \in X(\xi)} \bar{\lambda}^{T} f(x, \xi)=0,
$$

which, by the choice of $\bar{\lambda}$, is equivalent to

$$
\bar{\lambda}^{T} \bar{z}-\sup _{\xi \in \mathcal{U}} \min _{x \in X(\xi)} \bar{\lambda}^{T} f(x, \xi)=0 .
$$

Let $\alpha:=\sum_{i=1}^{p}\left\|e_{i}\right\|$, where $e_{i} \in \mathbb{R}^{p}$ is the $i$-th unit vector and let $\lambda^{\text {min }}:=\min _{i=1, \ldots, p} \bar{\lambda}_{i}$. Using (14), we show that there exists $\bar{\xi} \in \mathcal{U}$ such that $\operatorname{dist}\left(\{\bar{z}\}, \mathcal{Y}_{P}(\bar{\xi})\right)<\frac{\varepsilon}{2}$. We set $\bar{\varepsilon}:=\varepsilon \cdot \frac{\lambda^{m i n}}{2 \alpha}>0$ and by $(14)$, there exists $\bar{\xi} \in \mathcal{U}$ such that

$$
\bar{\lambda}^{T} \bar{z}-\min _{x \in X(\bar{\xi})} \bar{\lambda}^{T} f(x, \bar{\xi})<\bar{\varepsilon} .
$$

By Corollary 5 , there exists $\bar{y} \in \mathcal{Y}_{P}(\bar{\xi})$ such that $\bar{y} \leqq \bar{z}$. Moreover, because $\mathcal{Y}(\bar{\xi})$ is $\mathbb{R}_{\geq-}^{p}$ convex, we obtain for the half space $H:=\left\{y \in \mathbb{R}^{p} \mid \bar{\lambda}^{T} y \geq \min _{y \in \mathcal{Y}(\bar{\xi})} \bar{\lambda}^{T} y\right\}$ that $\bar{y} \in$ $\left(\{\bar{z}\}-\mathbb{R}_{\geqq}^{p}\right) \cap H$ and consequently, using Definition 8,

$$
\operatorname{dist}\left(\{\bar{z}\}, \mathcal{Y}_{P}(\bar{\xi})\right)=\inf _{y \in \mathcal{Y}_{P}(\bar{\xi})}\|\bar{z}-y\| \leq\|\bar{z}-\bar{y}\| \leq \max _{y \in\left(\{\bar{z}\}-\mathbb{R}_{\geqq}^{p}\right) \cap H}\|\bar{z}-y\| .
$$

For each $y \in\left(\{\bar{z}\}-\mathbb{R}_{\geqq}^{p}\right) \cap H$, by the choice of $\bar{\xi}$ in (15), we have

$$
\lambda^{\min } \sum_{i=1}^{p}\left(\bar{z}_{i}-y_{i}\right) \leq \sum_{i=1}^{p} \bar{\lambda}_{i}\left(\bar{z}_{i}-y_{i}\right)=\bar{\lambda}^{T}(\bar{z}-y) \leq \bar{\lambda}^{T} \bar{z}-\min _{y \in H} \bar{\lambda}^{T} y=\bar{\lambda}^{T} \bar{z}-\min _{y \in \mathcal{Y}(\bar{\xi})} \bar{\lambda}^{T} y<\bar{\varepsilon},
$$

since $\left(\bar{z}_{i}-y_{i}\right) \geq 0$ for all $i=1, \ldots, p$, and hence,

$$
\max _{i=1, \ldots, p}\left|\bar{z}_{i}-y_{i}\right|<\frac{\bar{\varepsilon}}{\lambda^{\min }} .
$$

As a consequence, we obtain

$$
\begin{aligned}
\operatorname{dist}\left(\{\bar{z}\}, \mathcal{Y}_{P}(\bar{\xi})\right) & \leq \max _{y \in\left(\{\bar{z}\}-\mathbb{R}_{\geqq}^{p}\right) \cap H}\|\bar{z}-y\| \\
\leq \max _{y \in\left(\{\bar{z}\}-\mathbb{R}_{\geqq}^{p}\right) \cap H} \sum_{i=1}^{p}\left|\bar{z}_{i}-y_{i}\right|\left\|e_{i}\right\| & \leq \max _{y \in\left(\{\bar{z}\}-\mathbb{R}_{\geqq}^{p}\right) \cap H} \max _{i=1, \ldots, p}\left|\bar{z}_{i}-y_{i}\right| \alpha \\
<\bar{\varepsilon} \frac{\alpha}{\lambda^{\text {min }}} & =\frac{\varepsilon}{2},
\end{aligned}
$$


where the first inequality follows from (16) and the second inequality follows from the triangle inequality of the primal norm $\|\cdot\|$. We then obtain, by the triangle inequality,

$$
\operatorname{dist}\left(\left\{z^{*}\right\}, \mathcal{Y}_{P}(\bar{\xi})\right)=\inf _{y \in \mathcal{Y}_{P}(\bar{\xi})}\left\|z^{*}-y\right\| \leq\left\|z^{*}-\bar{z}\right\|+\operatorname{dist}\left(\{\bar{z}\}, \mathcal{Y}_{P}(\bar{\xi})\right)<\frac{\varepsilon}{2}+\frac{\varepsilon}{2}=\varepsilon
$$

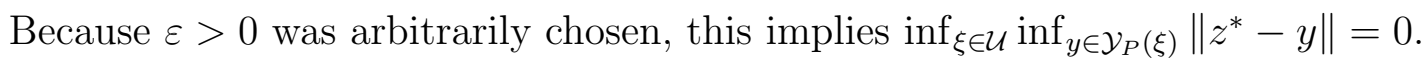

Under Assumption 1, we obtain a result for convex robust multiobjective optimization that generalizes [BTN98, Th. 2.1].

Theorem 23. Let the Continuity Assumption, the Convexity Assumption and Assumption 1 hold. Then

(a) The robust counterpart $\mathrm{RC}$ is feasible if and only if all instances of $\{\mathrm{P}(\xi)\}_{\xi \in \mathcal{U}}$ are feasible.

(b) If $f^{\mathrm{RC}}$ is given as in (4) then the robustness gap vanishes, i.e., $\vartheta=0$.

Proof. Part (a) follows directly from [BTN98, Th. 2.1] since the definition of robust feasibility in [BTN98] is the one used in this work.

Part (b) follows directly from Definition 8 and Proposition 22

$$
\vartheta=\inf _{\xi \in \mathcal{U}} \inf _{z \in \mathcal{Y}_{P}^{\mathrm{RC}}} \inf _{y \in \mathcal{Y}_{P}(\xi)}\|z-y\|=\inf _{z \in \mathcal{Y}_{P}^{\mathrm{RC}}}\left(\inf _{\xi \in \mathcal{U}} \inf _{y \in \mathcal{Y}_{P}(\xi)}\|z-y\|\right)=\inf _{z \in \mathcal{Y}_{P}^{\mathrm{RC}}} 0=0,
$$

where the second equality holds because all three infima are finite since $\|\cdot\|$ is bounded from below by 0 .

\section{Approximating the robustness gap for linear prob- lems}

In this section, we study $\{\mathrm{P}(\xi)\}_{\xi \in \mathcal{U}}$ in the linear form under two types of uncertainty, decision uncertainty and parameter uncertainty. In multiobjective linear programs (MOLPs) decision uncertainty can be contained in the vector of decision variables $x \in \mathbb{R}^{n}$ and parameter uncertainty can be contained in the problem data $(C, A, b) \in \mathbb{R}^{p \times n} \times \mathbb{R}^{m \times n} \times \mathbb{R}^{m}$. In the following, we separately discuss uncertain MOLPs that are affected by either kind of uncertainty or both.

\subsection{Decision Uncertainty}

Decision uncertainty in MOLPs is first discussed and an optimization problem for computing $\Delta^{L}$ is developed. Decision uncertainty accounts for the difference between a calculated 
solution and the way that solution is put into practice. For single-objective robust optimization problems, decision uncertainty has been studied under various names, see, e.g., [BTN02, BNT07, Das00, LP09, SdH08]. It is also known as implementation error.

We assume that the realization of a decision vector $x \in \mathbb{R}^{n}$ is an element of the set $\{x\}+\mathcal{U}$, where $\mathcal{U} \subseteq \mathbb{R}^{n}$ is a compact uncertainty set. This setting leads to the following uncertain MOLP

$$
\left\{\begin{array}{rrr}
(\operatorname{MOLP}(\xi)) & \min C(x+\xi) & \\
& \text { s. t. } A(x+\xi) & \geqq b \\
& & \in \mathbb{R}^{n}
\end{array}\right\}_{\xi \in \mathcal{U}} .
$$

The following concept addresses robust feasiblity in case of decision uncertainty in MOLPs. This definition of decision robust feasibility for $\{\operatorname{MOLP}(\xi)\}_{\xi \in \mathcal{U}}$ fits Definition 2 .

Definition 24. A point $x^{*} \in \mathbb{R}^{n}$ is called a decision robust feasible solution to $\{\operatorname{MOLP}(\xi)\}_{\xi \in \mathcal{U}}$ if all possible realizations of $x^{*}$ are feasible, i.e., if

$$
x^{*} \in X^{\mathrm{RC}}:=\left\{x \in \mathbb{R}^{n} \mid A(x+\xi) \geqq b, \forall \xi \in \mathcal{U}\right\} .
$$

The set $X^{\mathrm{RC}}$ is called the decision robust feasible set.

For a thorough study of a solution concept for decision uncertainty in multiobjective optimization, we refer the reader to [EKS17], and we use the robust counterpart defined therein. Note that in this setting the robust counterpart of $\{\operatorname{MOLP}(\xi)\}_{\xi \in \mathcal{U}}$ is a set-valued optimization problem. However, according to [EKS17, Th. 22], for the special case of decision uncertainty in MOLPs, the robust counterpart of $\{\operatorname{MOLP}(\xi)\}_{\xi \in \mathcal{U}}$ reduces to an MOLP of the form

$$
\begin{array}{ll}
\left(\text { DecRC }^{\mathrm{MOLP}}\right) & \min \\
& C x \\
\text { s. t. } & x \in X^{\mathrm{RC}},
\end{array}
$$

where the objective function is exactly the deterministic objective function of $\{\operatorname{MOLP}(\xi)\}_{\xi \in \mathcal{U}}$ and $X^{\mathrm{RC}}$ is defined as in Definition 24. In the following, DecRC ${ }^{\mathrm{MOLP}}$ is called the decision robust counterpart of $\{\operatorname{MOLP}(\xi)\}_{\xi \in \mathcal{U}}$ and a solution $x^{*} \in X^{\mathrm{RC}}$ is called a decision robust (Pareto-)efficient solution of $\{\operatorname{MOLP}(\xi)\}_{\xi \in \mathcal{U}}$ if it is an efficient solution to DecRC ${ }^{\mathrm{MOLP}}$ in the sense of Definition 1, i.e., if there is no $x \in X^{\mathrm{RC}}$ with the property $C x \leq C x^{*}$.

Note that DecRC ${ }^{\mathrm{MOLP}}$ is a semi-infinite optimization probem with the decision uncertainty in DecRC ${ }^{\text {MOLP }}$ contained only in the constraints, which are linear inequalities by Definition 24. According to [BTGN09, Th. 1.3.4], the set $X^{\mathrm{RC}}$ can be equivalently represented as a finite system of linear inequalities.

In order to construct a finite representation of the linear inequalities defining $X^{\mathrm{RC}}$, the inequalities are first reformulated. Let $\mathcal{U}^{\prime} \subseteq \mathbb{R}^{m}$ be defined as $\mathcal{U}^{\prime}:=\{b-A \xi \mid \xi \in \mathcal{U}\}$, then

$$
X^{\mathrm{RC}}=\left\{x \in \mathbb{R}^{n} \mid A x \geqq \xi, \forall \xi \in \mathcal{U}^{\prime}\right\} .
$$

Because the uncertainty set $\mathcal{U}$ is assumed to be compact, the uncertainty set $\mathcal{U}^{\prime}$ is also compact and $\max _{\xi \in \mathcal{U}^{\prime}} \xi_{i}$ exists for all $1 \leq i \leq m$. Hence, the vector $\tilde{b}:=\left(\max _{\xi \in \mathcal{U}^{\prime}} \xi_{1}, \ldots, \max _{\xi \in \mathcal{U}^{\prime}} \xi_{m}\right) \in$ 
$\mathbb{R}^{m}$ is well-defined. Then the set $X^{\mathrm{RC}}$ can equivalently be represented as

$$
X^{\mathrm{RC}}=\left\{x \in \mathbb{R}^{n} \mid A x \geqq \tilde{b}\right\},
$$

which is a finite system of linear inequalities. Therefore, DecRC ${ }^{\mathrm{MOLP}}$ with $X^{\mathrm{RC}}$ as in (18) reduces to an MOLP if $\{\operatorname{MOLP}(\xi)\}_{\xi \in \mathcal{U}}$ carries decision uncertainty.

Using the specific setting of decision uncertainty in MOLPs and (18), an additional condition for the robustness gap being zero is provided in the following result.

Proposition 25. Let $X^{\mathrm{RC}}$ in DecRC $\mathrm{MOLP}^{\mathrm{m}}$ be given by (18). If there exists an $(n-1)$ dimensional face $\mathcal{F}$ of the polyhedral set $X^{\mathrm{RC}}$ such that $\mathcal{F} \subseteq X_{E}^{\mathrm{RC}}$, then

$$
\vartheta=0
$$

and there exists a scenario $\bar{\xi} \in \mathcal{U}$ and an $(n-1)$-dimensional face $\mathcal{F}^{\prime}$ of the polyhedral set $X(\bar{\xi})$ such that $\mathcal{F}^{\prime} \subseteq X_{E}(\bar{\xi})$.

Proof. By [KL00, Cor. 5.7], $\mathcal{F} \subseteq X_{E}^{\mathrm{RC}}$ if and only if there exist $1 \leq j \leq m$ such that

$$
\mathcal{F}=\left\{x \in \mathbb{R}^{n} \mid A_{j} x=\tilde{b}_{j}, A_{i} x \geq \tilde{b}_{i} \forall 1 \leq i \leq m\right\}
$$

where $A_{i}$ is the $i$-th row of $A$ for all $1 \leq i \leq m$, and $\mu \in \mathbb{R}_{>}^{p}$ such that

$$
A_{j}=\sum_{i=1}^{p} \mu_{i} C_{i},
$$

where $C_{i}$ is the $i$-th row of $C$ for all $1 \leq i \leq p$.

Because $\mathcal{U}$ is compact, it holds $\sup _{\xi \in \mathcal{U}^{\prime}} \xi_{j}=\max _{\xi \in \mathcal{U}} \xi_{j}$ and there exists $\bar{\xi} \in \mathcal{U}$ such that $\tilde{b}_{j}=\max _{\xi \in \mathcal{U}} \xi_{j}=\bar{\xi}_{j}$. For every $x \in \mathcal{F}$ and every $1 \leq i \leq m$ we have $A_{i} x \geq \tilde{b}_{i} \geq \bar{\xi}_{i}$ and hence

$$
\mathcal{F} \subseteq \mathcal{F}^{\prime}:=\left\{x \in \mathbb{R}^{n} \mid A_{j} x=\tilde{b}_{j}, A_{i} x \geq \bar{\xi}_{i} \forall 1 \leq i \leq m\right\} \subseteq X(\bar{\xi}) .
$$

Applying the reverse direction of [KL00, Cor. 5.7] to both $\mathcal{F}^{\prime}$ and (19), we obtain $\mathcal{F}^{\prime} \subseteq$ $X_{E}(\bar{\xi})$ and hence, $\mathcal{F} \subseteq \mathcal{F}^{\prime} \subseteq X_{E}(\bar{\xi})$. Therefore, $\mathcal{F} \subseteq X_{E}^{\mathrm{RC}} \cap X_{E}(\bar{\xi})$ and, because $f^{\mathrm{RC}}(x)=$ $f(x, \bar{\xi})=C x$ for all $x \in \mathbb{R}^{n}$, applying Definition 8 , we obtain $\vartheta=0$.

We now show that the lower bound $\Delta^{L}$ on the robustness gap $\vartheta$ may be computed as the optimal value of a DC optimization problem, if the uncertainty set $\mathcal{U}$ is a convex polytope, the matrix $C \in \mathbb{R}^{p \times n}$ is positive semidefinite, and the primal norm is a block norm, i.e., the unit ball of the norm is a polyhedral set. In particular, the objective function of this optimization problem is a difference of two convex functions and the constraints are linear functions.

Note that the uncertainty set $\mathcal{U}^{\prime}=\{b-A \xi \mid \xi \in \mathcal{U}\}$ is also a convex polytope whenever $\mathcal{U}$ is a convex polytope. Hence, we assume that the uncertainty set is given as $\mathcal{U}^{\prime}=\{\xi \in$ $\left.\mathbb{R}^{m} \mid D \xi \geqq d\right\}$, where $D \in \mathbb{R}^{t \times m}$ and $d \in \mathbb{R}^{t}$. 
By Definition 10, the lower bound $\Delta^{L}$ is given as

$$
\Delta^{L}=\inf _{\xi \in \mathcal{U}^{\prime}} \min _{\substack{\lambda \in \mathbb{R}_{\geq}^{p} \\
\|\lambda\|_{*}=1}}\left(\begin{array}{cccc}
\min _{x} & \lambda^{T} C x & -\min _{x} & \lambda^{T} C x \\
\text { s. t. } & A x \geqq \tilde{b} & \text { s. t. } & A x \geqq \xi
\end{array}\right),
$$

where (18) is used in the left hand problem. Applying linear programming duality to the right hand problem, we obtain

$$
\Delta^{L}=\inf _{\xi \in \mathcal{U}^{\prime}} \min _{\substack{\lambda \in \mathbb{R}_{\geq}^{p} \\
\|\lambda\|_{*}=1}}\left(\begin{array}{ccccc}
\min _{x} & \lambda^{T} C x & -\max _{v} & v^{T} \xi \\
\text { s.t. } & A x \geqq \tilde{b} & \text { s. t. } & v^{T} A=\lambda^{T} C \\
& & & v \geqq 0
\end{array}\right) .
$$

Finally, assuming the infimum can be replaced by the minimum, the following formulation is obtained

$$
\Delta^{L}=\left(\begin{array}{rl}
\min _{x, v, \lambda, \xi} \lambda^{T} C x & -v^{T} \xi \\
\text { s. t. } \quad A x & \geqq \tilde{b} \\
D \xi & \geqq d \\
v^{T} A & =\lambda^{T} C \\
\|\lambda\|_{*} & =1 \\
\lambda, v & \geqq 0
\end{array}\right),
$$

which becomes a DC problem if $C$ is positive semidefinite and the primal norm (and hence its dual) is a block norm. Computing the block norm of a point can be formulated as linear constraints using characterizations of [WW85] see, e.g., also [CGS17] when applied to robust optimization.

For the upper bound $\Delta^{U}$, using Definition 10 we proceed in a similar way. Applying linear programming duality to the left hand problem in

$$
\Delta^{U}=\inf _{\xi \in \mathcal{U}^{\prime}} \max _{\substack{\lambda \in \mathbb{R}_{\geq}^{p} \\
\|\lambda\|_{*}=1}}\left(\begin{array}{cccc}
\min _{x} & \lambda^{T} C x & -\min _{x} & \lambda^{T} C x \\
\text { s. t. } & A x \geqq \tilde{b} & \text { s. t. } & A x \geqq \xi
\end{array}\right),
$$

we obtain

$$
\Delta^{U}=\inf _{\xi \in \mathcal{U}^{\prime}}\left(\begin{array}{cc}
\max _{x, u, \lambda} & u^{T} \tilde{b}-\lambda^{T} C x \\
\text { s.t. } & A x \\
& \geqq \xi \\
u^{T} A & =\lambda^{T} C \\
\|\lambda\|_{*} & =1 \\
\lambda, u & \geqq 0
\end{array}\right),
$$

which is a bilevel problem that has a quadratic objective function on the lower level. 


\subsection{Parameter Uncertainty}

Parameter uncertainty in MOLPs is now investigated and an optimization problem for computing $\Delta^{L}$ is given. Parameter uncertainty accounts for uncertainty in the data of optimization problems. Hence, both the linear objective and the linear constraints are considered to be uncertain and the scenarios in the uncertainty set are written as $\xi:=$ $(C, A, b) \in \mathcal{U}$, where $(C, A, b) \in \mathbb{R}^{p \times n} \times \mathbb{R}^{m \times n} \times \mathbb{R}^{m}$ and where the matrices $C$ and $A$ consist of the rows $C_{i} \in \mathbb{R}^{n}$ for $i=1, \ldots, p$ and $A_{j} \in \mathbb{R}^{n}$ for $j=1, \ldots, m$, respectively. This setting leads to the following uncertain MOLP

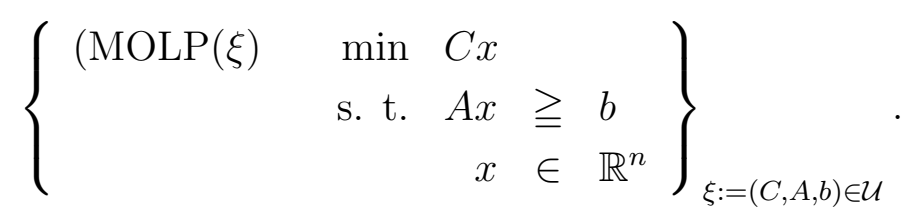

Note that MOLPs with decision uncertainty can be considered as MOLPs with parameter uncertainty as defined here where only the right-hand side $b \in \mathbb{R}^{m}$ is affected by uncertainty, see (18) in Section 4.1. Hence, this section covers not only the case of MOLPs that are affected by either uncertainty but also the case of MOLPs containing both types of uncertainty.

In the following MOLPs with parameter uncertainty are treated as a special cases of uncertain MOPs, as introduced in Section 1, and so robust feasibility for $\{\operatorname{MOLP}(\xi)\}_{\xi \in \mathcal{U}}$ follows from Definition 2.

Definition 26. A point $x^{*} \in \mathbb{R}^{n}$ is called a robust feasible solution to $\{\operatorname{MOLP}(\xi)\}_{\xi \in \mathcal{U}}$ if $x^{*}$ is feasible for all scenarios $(C, A, b) \in \mathcal{U}$, i.e., if

$$
x^{*} \in X^{\mathrm{RC}}:=\left\{x \in \mathbb{R}^{n} \mid A x \geqq b, \forall \xi=(C, A, b) \in \mathcal{U}\right\} .
$$

The robust counterpart of $\{\operatorname{MOLP}(\xi)\}_{\xi \in \mathcal{U}}$ makes use of the concepts (3) and (4) in Section 1. We obtain

$$
\begin{aligned}
\left(\mathrm{RC}^{\mathrm{MOLP}}\right) \quad \min & \left(\sup _{\xi \in \mathcal{U}} C_{1} x, \ldots, \sup _{\xi \in \mathcal{U}} C_{p} x\right)^{T} \\
\text { s. t. } & x \in X^{\mathrm{RC}}
\end{aligned}
$$

where $\sup _{\xi \in \mathcal{U}} C_{i} x=\max _{\xi \in \mathcal{U}} C_{i} x$ for all $1 \leq i \leq p$ because $\mathcal{U}$ is a compact set. Because $\mathrm{RC}^{\mathrm{MOLP}}$ is an MOP, we can directly apply the classical definition of Pareto efficiency as is also done in Definition 3 for general uncertain MOPs. By Definition 3, a solution $x^{*} \in X^{\mathrm{RC}}$ is called a robust (Pareto-)efficient solution of $\{\operatorname{MOLP}(\xi)\}_{\xi \in \mathcal{U}}$ if it is an efficient solution to $\mathrm{RC}^{\mathrm{MOLP}}$ in the sense of Definition 1, i.e., if there is no $x \in X^{\mathrm{RC}}$ with the property

$$
\left(\sup _{\xi \in \mathcal{U}} C_{1} x, \ldots, \sup _{\xi \in \mathcal{U}} C_{p} x\right)^{T} \leq\left(\sup _{\xi \in \mathcal{U}} C_{1} x^{*}, \ldots, \sup _{\xi \in \mathcal{U}} C_{p} x^{*}\right)^{T} .
$$

This approach is found often in the literature, see, e.g., [KL12, FW14, GJLVP14, Chu16]. 
The robust counterpart $\mathrm{RC}^{\mathrm{MOLP}}$ can equivalently be rewritten such that only the constraints are affected by uncertainty

$$
\begin{array}{rrrr}
\left(\mathrm{RC}^{\mathrm{MOLP}}\right) & \min & \\
\text { s. t. } & -C x+\tau & \geqq 0 & \forall(C, A, b) \in \mathcal{U} \\
& A x \geqq b & \forall(C, A, b) \in \mathcal{U}
\end{array}
$$

where $\tau \in \mathbb{R}^{p}$, which has commonly been used in single-objective reformulations, see, e.g., [BTGN09], and multiobjective reformulations, see, e.g., [FW14, GJLVP14].

In order to simplify the notation, we can further reformulate $\mathrm{RC}^{\mathrm{MOLP}}$ by defining a new uncertainty set

$$
\mathcal{U}^{\prime}:=\left\{\xi:=\left(A^{\prime}, b^{\prime}\right) \in \mathbb{R}^{(p+m) \times(n+p+1)} \mid A^{\prime}=\left(\begin{array}{cc}
-C & \mathrm{I}_{p} \\
A & 0
\end{array}\right), b^{\prime}=\left(\begin{array}{l}
0 \\
b
\end{array}\right),(C, A, b) \in \mathcal{U}\right\}
$$

where $\mathrm{I}_{p} \in \mathbb{R}^{p \times p}$ is the identity matrix. Letting $\bar{C}:=\left(\begin{array}{ll}0 & \mathrm{I}_{p}\end{array}\right) \in \mathbb{R}^{p \times(n+p)}$ and $x^{\prime}:=$ $(x, \tau)^{T} \in \mathbb{R}^{n+p}, \mathrm{RC}^{\mathrm{MOLP}}$ can be rewritten as

$$
\begin{array}{ll}
\left(\mathrm{RC}^{\mathrm{MOLP}}\right) & \min \quad \bar{C} x^{\prime} \\
& \text { s. t. } A^{\prime} x^{\prime} \geqq b^{\prime} \quad \forall\left(A^{\prime}, b^{\prime}\right) \in \mathcal{U}^{\prime} .
\end{array}
$$

The robust counterpart $\mathrm{RC}^{\mathrm{MOLP}}$ in $(20)$ is a semi-infinite problem that carries uncertainty only in the constraints. Therefore, results from single-objective robust optimization can be applied and, according to [BTGN09, Cor. 1.3.5], the constraints of $\mathrm{RC}^{\mathrm{MOLP}}$ can be represented as a finite system of linear inequalities if $\mathcal{U}$ is a polyhedral set.

Considering the inequality system $\bar{A} x^{\prime} \geqq \bar{b}$ as the corresponding finite representation of the system $A^{\prime} x \geqq b^{\prime}$ for all $\left(A^{\prime}, b^{\prime}\right) \in \mathcal{U}^{\prime}$, we obtain

$$
\begin{array}{ll}
\left(\mathrm{RC}^{\mathrm{MOLP}}\right) & \min \quad \bar{C} x^{\prime} \\
& \text { s. t. } \bar{A} x^{\prime} \geqq \bar{b} .
\end{array}
$$

Analogously to Section 4.1, we now develop an optimization problem to compute $\Delta^{L}$. If the uncertainty set $\mathcal{U}$ is a convex polytope, the matrix $\bar{C} \in \mathbb{R}^{p \times n}$ is positive semidefinite, and the primal norm is a block norm then the lower bound $\Delta^{L}$ of $\vartheta$ may be computed as the optimal objective value of a DC optimization problem. Note that the uncertainty set $\mathcal{U}^{\prime}$ is also a convex polytope whenever $\mathcal{U}$ is a convex polytope. Hence, we assume that the uncertainty set is given as

$$
\mathcal{U}^{\prime}=\left\{\left(A^{\prime}, b^{\prime}\right) \in \mathbb{R}^{(p+m) \times(n+p+1)} \mid \sum_{i=1}^{p+m} \sum_{j=1}^{n+p+1} D_{k i j}\left(A^{\prime}, b^{\prime}\right)_{i j} \geqq d_{k}, 1 \leq k \leq t\right\},
$$

where $D \in \mathbb{R}^{t \times(p+m) \times(n+p)}$ and $d \in \mathbb{R}^{t}$. 
By Definition 10, the lower bound $\Delta^{L}$ is given as

$$
\Delta^{L}=\inf _{\left(A^{\prime}, b^{\prime}\right) \in \mathcal{U}^{\prime}} \min _{\substack{\lambda \in \mathbb{R}_{\geq}^{p} \\
\|\lambda\|_{*}=1}}\left(\begin{array}{cccc}
\min _{x} & \lambda^{T} \bar{C} x & -\min _{x} & \lambda^{T} \bar{C} x \\
\text { s. t. } & \bar{A} x \geqq \bar{b} & \text { s.t. } & A^{\prime} x \geqq b^{\prime}
\end{array}\right),
$$

where (21) is used in the left hand problem. Applying linear programming duality to the right hand problem, we obtain

$$
\Delta^{L}=\inf _{\left(A^{\prime}, b^{\prime}\right) \in \mathcal{U}^{\prime}} \min _{\substack{\lambda \in \mathbb{R}_{\geq}^{p} \\
\|\lambda\|_{*}=1}}\left(\begin{array}{cccc}
\min _{x} & \lambda^{T} \bar{C} x & -\max _{v} & v^{T} b^{\prime} \\
\text { s.t. } & \bar{A} x \geqq \bar{b} & \text { s.t. } & v^{T} A^{\prime}=\lambda^{T} \bar{C} \\
& & & v \geqq 0
\end{array}\right) .
$$

Finally, assuming the infimum can be replaced by the minimum, the following formulation is obtained

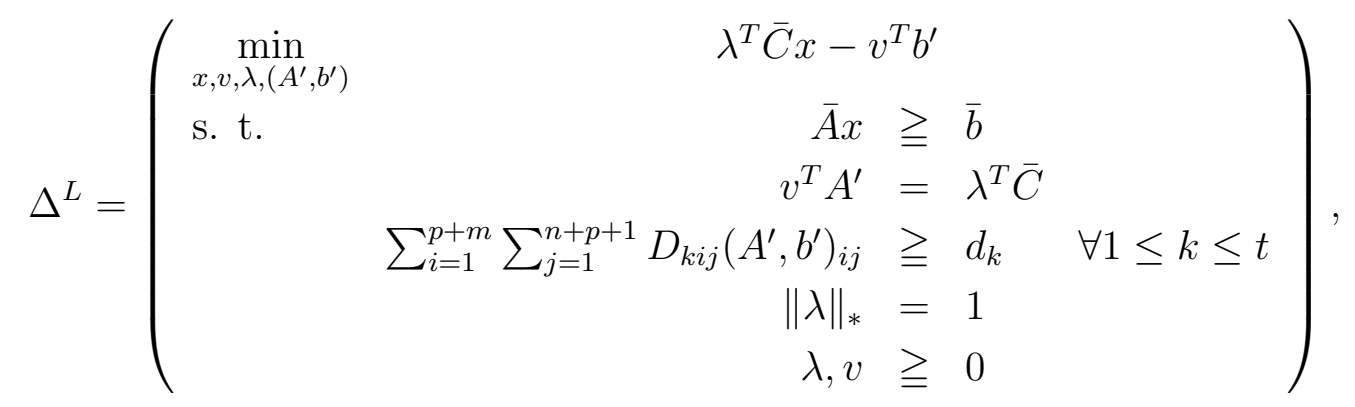

which becomes a DC problem if $C$ is positive semidefinite and if the dual norm $\|\cdot\|_{*}$ is a block norm.

For the upper bound $\Delta^{U}$, using Definition 10 we proceed in a similar way. Applying linear programming duality to the left hand problem in

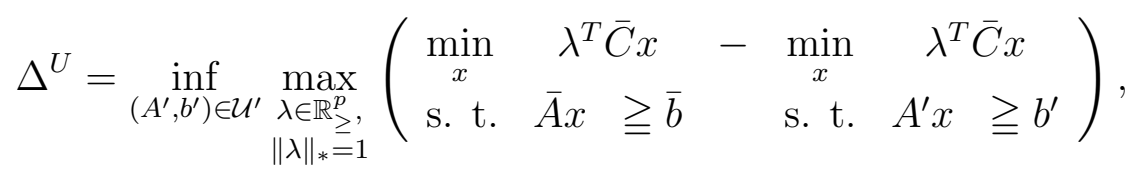

we obtain

$$
\Delta^{U}=\inf _{\left(A^{\prime}, b^{\prime}\right) \in \mathcal{U}}\left(\begin{array}{cc}
\max _{x, u, \lambda} & u^{T} \bar{b}-\lambda^{T} \bar{C} x \\
\text { s. t. } \quad & A^{\prime} x \\
u^{T} \bar{A} & \geqq b^{\prime} \\
\|\lambda\|_{*} & =1 \\
\lambda, u & \geqq 0
\end{array}\right),
$$

which is a bilevel problem that has a quadratic objective function on the lower level. 


\section{Conclusions}

A concept of a robustness gap for uncertain multiobjective optimization is introduced that is applicable to the most often studied robustness concepts in the literature. The gap is defined as the minimal distance between the robust Pareto set and the Pareto sets of the scenarios.

Because Pareto sets are typically nonconvex even for linear programs and computing a minimal distance between them is hard, lower and upper bounds for the robustness gap are derived for the case of convex MOPs, using the weighted sum scalarization. Due to the nature of this scalarization, the bounds are computed by solving a single-objective problem in both the objective space and its dual space. A sufficient condition for the upper bound to be valid is developed. This condition is satisfied by all pairs of $\mathfrak{p}$ and $\mathfrak{q}$-norms with $\mathfrak{p}, \mathfrak{q} \in[1, \infty]$ under an additional assumption, and it is always satisfied in the two-dimensional objective space. The condition and the upper bound are also related to normal cones and restricted normal cones as studied in the literature.

The proposed multiobjective robustness gap is related to the classical robustness gap concept in the single-objective case. The well-known result about the single-objective zero robustness gap is generalized for the multiobjective robustness gap, i.e., the multiobjective robustness gap is zero whenever the uncertainty is constraint-wise and objective-wise. Moreover, it is shown that for every element in the robust Pareto set there exists a scenario whose Pareto set is arbitrarily close to that specific robust Pareto point.

More results are obtained for the case of linear problems. For MOLPs with decision uncertainty, the robustness gap is zero if there exists an $(n-1)$-dimensional robust efficient face of the robust feasible set. In the case of MOLPs with decision uncertainty or parameter uncertainty, the lower bound can be computed by methods from DC programming for polyhedral uncertainty sets and block norms on the objective space.

Note that the presented concept of the multiobjective robustness gap is applicable to all robustness concepts for uncertain MOPs whose robust counterpart is an MOP. The proposed gap can also be applied to to other robustness concepts such as flimsily robust efficiency and highly robust efficiency as defined in [IS16]. Due to the nature of these two concepts, the robustness gap is always zero for flimsily robust efficiency and it is zero for highly robust efficiency whenever a highly efficient solution exists.

There are different areas of interest for further research. First, it is of interest to eliminate the additional assumption that is needed for the sufficient condition for the upper bound to hold in case of pairs of $\mathfrak{p}$ and $\mathfrak{q}$-norms with $\mathfrak{p}, \mathfrak{q} \in(1, \infty)$. Second, dependence of the bounds for the robustness gap on the type of the scalarization used in their derivation could be analyzed. Third, the robustness gap may require a different definition if an ordering cone for an uncertain MOP is not the Pareto cone $\mathbb{R}_{\geqq}^{p}$ but another suitable cone as used for robust multiobjective optimization in $\left[\mathrm{IKK}^{+} 14\right]$. For the latter, different scalarization techniques may be beneficial. Finally, a robustness gap could be proposed for those concepts of robustness for uncertain MOPs that do not define the robust counterpart as an MOP but rather as a set-valued optimization problem, see e.g., [EKS17, EIS14]. However, in case of objective-wise uncertainty, the set-valued robust counterpart can be reduced to an 
MOP, see [EIS14, Th. 5.4] and the presented concept of the multiobjective robustness gap remains applicable.

\section{References}

[Ber63] C. Berge. Topological spaces: including a triatment of mltivalued functions, vector spaces and convexity. Oliver and Boyd, 1963.

[BF17] R. Bokrantz and A. Fredriksson. Necessary and sufficient conditions for pareto efficiency in robust multiobjective optimization. European Journal of Operational Research, 262(2):682 - 692, 2017.

[BGW09] R.I. Bot, S.-M. Grad, and G. Wanka. Duality in Vector Optimization. Vector Optimization. Springer, 2009.

[BLPW13] H. H. Bauschke, D. R. Luke, H. M. Phan, and X. Wang. Restricted normal cones and the method of alternating projections: Theory. Set-Valued and Variational Analysis, 21(3):431-473, 2013.

[BNT07] D. Bertsimas, O. Nohadani, and K. M. Teo. Robust optimization in electromagnetic scattering problems. Journal of Applied Physics, 101(7):074507, 2007.

[BS99] H.P. Benson and E. Sun. New closedness results for efficient sets in multiple objective mathematical programming. Journal of Mathematical Analysis and Applications, 238(1):277 - 296, 1999.

[BTGN09] A. Ben-Tal, L. El Ghaoui, and A. Nemirovski. Robust Optimization. Princeton University Press, Princeton and Oxford, 2009.

[BTN98] A. Ben-Tal and A. Nemirovski. Robust convex optimization. Mathematics of Operations Research, 23(4):769-805, 1998.

[BTN99] A. Ben-Tal and A. Nemirovski. Robust solutions of uncertain linear programs. Operations Research Letters, 25:1-13, 1999.

[BTN02] A. Ben-Tal and A. Nemirovski. Robust optimization - methodology and applications. Mathematical Programming, 92(3):453-480, 2002.

[CGS17] E. Carrizosa, M. Goerigk, and A. Schöbel. A biobjective approach to recovery robustness based on location planning. European Journal of Operational Research, 261:421-435, 2017.

[Chu16] T. D. Chuong. Optimality and duality for robust multiobjective optimization problems. Nonlinear Analysis: Theory, Methods \&6 Applications, 134:127-143, 2016. 
[Cve12] Z. Cvetkovski. Inequalities: Theorems, Techniques and Selected Problems. Springer Berlin Heidelberg, 2012.

[Das00] Indraneel Das. Robustness opimization for constrained nonlinear programming problems. Engineering Optimization+ A35, 32(5):585-618, 2000.

[DKW12] E.K. Doolittle, H.L. M. Kerivin, and M. M. Wiecek. A robust multiobjective optimization problem with application to internet routing. Technical Report R2012-11-DKW, Clemson University, 2012.

[Ehr05] M. Ehrgott. Multicriteria Optimization. Springer Berlin Heidelberg, 2005.

[EIS14] M. Ehrgott, J. Ide, and A. Schöbel. Minmax robustness for multi-objective optimization problems. European Journal of Operational Research, 239:17-31, 2014.

[EKS17] G. Eichfelder, C. Krüger, and A. Schöbel. Decision uncertainty in multiobjective optimization. Journal of Global Optimization, 69(2):485-510, 2017.

[FW14] J. Fliege and R. Werner. Robust multiobjective optimization \& applications in portfolio optimization. European Journal of Operational Research, 234(2):422 $-433,2014$.

[Geo68] A. M. Geoffrion. Proper efficiency and the theory of vector maximization. Journal of Mathematical Analysis and Applications, 22(3):618 - 630, 1968.

[GJLVP14] M. A. Goberna, V. Jeyakumar, G. Li, and J. Vicente-Pérez. Robust solutions of multiobjective linear semi-infinite programs under constraint data uncertainty. SIAM Journal on Optimization, 24(3):1402-1419, 2014.

[Har78] R. Hartley. On cone-efficiency, cone-convexity and cone-compactness. SIAM Journal on Applied Mathematics, 34(2):211-222, 1978.

$\left[\mathrm{IKK}^{+} 14\right] \quad J$. Ide, E. Köbis, D. Kuroiwa, A. Schöbel, and C. Tammer. The relationship between multi-objective robustness concepts and set valued optimization. Fixed Point Theory and Applications, 2014(83), 2014.

[IS16] J. Ide and A. Schöbel. Robustness for uncertain multi-objective optimization: A survey and analysis of different concepts. OR Spectrum, 38(1):235-271, 2016.

[KL00] N. T. B. Kim and D. T. Luc. Normal cones to a polyhedral convex set and generating efficient faces in linear multiobjective programming. Acta Mathematica Vietnamica, 25(1), 2000.

[KL12] D. Kuroiwa and G. M. Lee. On robust multiobjective optimization. Vietnam Journal of Mathematics, 40(2\&3):305-317, 2012. 
[LP09] A.S. Lewis and C.H.J. Pang. Lipschitz behavior of the robust regularization. SIAM Journal on Control and Optimization, 48(5):3080-3105, 2009.

[Luc84] D. T. Luc. On the domination property in vector optimization. Journal of Optimization Theory and Applications, 43(2):327-330, 1984.

[Luc89] D. T. Luc. Theory of vector optimization, volume 319 of Lecture Notes in Economics and Mathematical Systems. Springer-Verlag, Berlin, 1989.

[Lue69] D.G. Luenberger. Optimization by Vector Space Methods. Professional Series. Wiley, 1969.

[Roc15] R. T. Rockafellar. Convex analysis. Princeton Landmarks in Mathematics and Physics. Princeton university press, 2015.

[SdH08] E. Stinstra and D. den Hertog. Robust optimization using computer experiments. European Journal of Operational Research, 191(3):816 - 837, 2008.

[WD16] M. M. Wiecek and G. M. Dranichak. Robust Multiobjective Optimization for Decision Making Under Uncertainty and Conflict, chapter 4, pages 84-114. INFORMS, 2016.

[WW85] J. E. Ward and R. E. Wendell. Using block norms for location modeling. Operations Research, 33(5):1074-1090, 1985. 


\section{Peat and Pots: An Application of Robust Multiobjective Optimization to a Mixing Problem in Agriculture}

C. Krüger, F. Castellani, J. Geldermann, A. Schöbel 
C. Peat and Pots: An Application of Robust Multiobjective Optimization to a Mixing Problem in Agriculture 


\title{
Peat and Pots: An application of robust multiobjective optimization to a mixing problem in agriculture
}

\author{
Corinna Krüger $^{* 1}$, Francesco Castellani ${ }^{2}$, Jutta Geldermann², and Anita Schöbel ${ }^{1}$ \\ ${ }^{1}$ Institute for Numerical and Applied Mathematics, University of Goettingen, \\ Lotzestr. 16-18, 37083 Göttingen, Germany \\ ${ }^{2}$ Institute for Economics, University of Goettingen, \\ Platz der Göttinger Sieben 3, 37073 Göttingen, Germany
}

\begin{abstract}
In horticultural settings, mixing problems arise when choosing a suitable growth substrate for potted plants. The aim of practitioners is to choose a pot and a growth substrate mixture such that environmental emissions and costs are simultaneously minimized. The impact of the plant's quality on the selling price should also be considered. The decision problem outlined here is affected by two types of uncertainty. First, the problem parameters are uncertain due to the nature of the input data. For example variations in the agronomic characteristics of the growth media and in the weather conditions lead to imprecision. Second, the composition of the growth media is generally not implemented exactly due to variability in the mixing process itself. If these uncertainties are ignored, the problem can be addressed using deterministic multiobjective optimization. If these uncertainties are to be considered, the problem can be addressed by means of robust multiobjective optimization. In this paper, we apply both a deterministic and a robust approach to the problem, with and without uncertainty. We present a case study of an Italian plant nursery and we compare deterministic and robust solutions. Our results show that the robust solutions are preferable to the deterministic ones, and that the robust approach is indeed worth considering for horticultural mixing problems and might also be used effectively in other settings where similar mixing decisions arise.
\end{abstract}

\section{Introduction}

Over the years, Operations Research has been used extensively to optimize environmentally friendly production planning in agricultural supply chain management Crespo et al. (2010); Bohle et al. (2010); Doole (2012). Multiobjective optimization is particularly helpful, since objectives other than profitability must also be taken into account (for a survey on multiobjective optimization in agriculture, see Hayashi (2000)). A specific challenge in handling renewable resources is the fluctuation in their quantity, quality, and material properties arising from the multiple-usage of by-products. Here, typical decision problems can be formulated as mixing problems, which have been quite thoroughly investigated Ashayeri et al. (1994); Shih and Frey (1995). In this work, however, we consider a specific mixing problem that is

${ }^{*}$ corresponding author; e-mail: ckrueger@math.uni-goettingen.de 
currently a challenge in the horticulture sector: replacing peat substrate and plastic pots with more environmentally-friendly alternatives.

Peat is a non-renewable resource widely used in horticulture due to its agronomic characteristics, which guarantee the quality of plants to be sold in the market. Although peat can indeed be replaced by compost - an agricultural by-product - the compost's variable agronomic properties determine different quality levels of a potted plant. Therefore, only a certain percentage of peat can be substituted in a blending process. A second way to improve the sustainability of horticulture would be to replace planter pots made of conventional plastic with innovative, biodegradable pots.

In mathematical terms, this problem thus comprises a binary decision between two types of pots and a decision about the share of compost used to partially replace the peat. Two objective functions are considered, one for the economic consequences and one for the ecological consequences:

- The economic consequences are the additional costs due to the processing steps associated with compost handling. The selling price of agricultural and horticultural products depends on their quality, a multi-faceted assessment often based on sampling, scoring, and quality ratings.

- The ecological consequence is the greenhouse gas reduction achieved by replacing peat (in part) with compost and plastic pots with biodegradable pots. This reduction can be determined by calculating the global warming potential (GWP) in $\mathrm{kg} \mathrm{CO}_{2}$ equivalents $\operatorname{IPCC}(2007)$.

The problem is characterized by two types of uncertainty that are typical in renewable resource processing: parameter uncertainty and decision uncertainty. The former is clearly caused by fluctuations in quality, quantity and material properties, but inaccuracies in experimental data and measurements also contribute Lowe and Preckel (2004); Geldermann et al. (2016). The latter occurs whenever solutions are not implemented exactly as calculated, but only within a range of the computed values. For example, the targeted share of compost is generally not achieved exactly, due to imprecisions in the mixing process.

In these situations, robust optimization provides a suitable framework for decision support, since it seeks solutions that are feasible for all scenarios and that perform best with respect to the worst case. Classic robustness concepts are minmax robustness Ben-Tal et al. (2009) and regret robustness Kouvelis and Yu (1997). Other concepts such as Bertsimas and Sim (2004); Schöbel (2014) are reviewed in Goerigk and Schöbel (2016). Robust optimization has been applied to various problems in agriculture. For example, Bohle et al. (2010) applied it to the scheduling of wine grape harvesting in Brazil; Doole (2012) applied it to compare deterministic and robust approaches to evaluating perennial pasture species in Australia by using uncertain parameters within intervals; Munhoz and Morabito (2014) applied it to orange juice production; and Hombach et al. (2017) applied a robust and multi-objective optimization model with integrated risk attitude to a biodiesel supply chain in Germany.

In this paper, we consider a minmax robustness approach for both parameter and decision uncertainty in multiobjective optimization (see Ehrgott et al. (2014); Kuroiwa and Lee (2012) for parameter uncertainty and see Eichfelder et al. (2017) for decision uncertainty).

Robust minmax multi-objective optimization results in rather complicated (sometimes setvalued) optimization problems (for recent surveys, see Ide and Schöbel (2016); Wiecek and Dranichak (2016)). Here, we use robust multiobjective optimization to determine solutions 
to our uncertain problem; the theoretical background for this work can be found in Krüger (2018). We also describe the managerial implications in a real-world multiobjective problem from the agricultural industry.

The paper is structured as follows. Section 2 describes the problem, which is initially analyzed and mathematically formulated without taking uncertainty into account. Section 3 illustrates a case study with its input data (environmental emissions and costs). In Section 4 and Section 5, the model is described in detail and a deterministic solution approach for the problem without uncertainty and a robust solution approach for the uncertain problem are displayed. In Section 6, we compare the deterministic and robust results and draw some conclusions about current horticulture management practices and promising avenues for further research.

\section{Problem description}

A major environmental problem in European horticulture is the greenhouse gas (GHG) emissions caused by potted plant cultivation using peat and plastics Lazzerini et al. (2014).

Peat accumulates under anaerobic conditions over thousands of years from partially decayed sphagnum, grasses, and other plants. It is a very flexible material that can be adapted for most plants, since it is generally low in nutrients, $\mathrm{pH}$, and bulk density. Moreover, it exhibits favorable cation exchange capacity and air-filled porosity characteristics Robbins and Evans (2001). Taken together, these parameters define the quality level of peat as a growth medium in horticulture. However, peatlands (or bogs) are important carbon sinks, and peat extraction results in significant emissions of carbon dioxide, methane, and nitrous oxide Waddington et al. (2009). Consequently, peatland protection aims to maintain both biodiversity and such ecosystem services as climate protection and nutrient retention. To reduce the environmental impacts of the sector, alternative materials (e.g., compost, bark, wood, rice hulls, animal manure) made of renewable resources or by-products can be substituted for peat.

In horticulture settings, peat could be best replaced by compost. Beyond a certain replacement percentage, however, the agronomic quality of the potted plant becomes unacceptable Papafotiou et al. (2005). Agricultural supply chains generate a great variety and quantity of waste and by-products that could be used as compost, including the by-products from the olive oil industry Raviv (2014).

In horticulture, plastic pots are widely used because of their low cost, durability, and versatility. Since they cannot be recycled easily due to soil and vegetable matter contamination, agrochemical residues, and additives, they are generally landfilled after one usage Schettini et al. (2013). In the United States, an estimated 750 thousand tons of plastic pots are disposed of annually Nambuthiri et al. (2015); in Italy, 91 thousand tons of plastic pots, sheets, and twines used in agriculture are disposed of annually Scarascia-Mugnozza et al. (2011). Here, biodegradable pots, which can be embedded in the soil with the plant or disposed of in composting facilities, represent a viable alternative to plastic pots.

From a responsible management perspective, it makes sent to consider both peat substitution and the replacement of plastic pots with biodegradable ones. The resulting reduction in GHG emissions can be determined by a Life Cycle Assessment (LCA), which looks at environmental impacts from all stages of a product's life, including raw material extraction, materials processing, manufacturing, distribution, use, repair, maintenance, and disposal or 
recycling Geldermann et al. (1999); Brandenburg et al. (2014).

Along with the goal of minimizing GHG emissions, one would also like to minimize the additional costs associated with substituting for peat and plastic pots. The additional costs comprise only those costs which are relevant for the decision, and that vary from the initial situation (i.e., the only use of peat and plastic containers to grow plants). This leads to a biobjective optimization problem, which is complicated by the two types of uncertainty mentioned in Section 1. Parameter uncertainty arises from lack of exact knowledge of cost and emission parameters. Here, one must work with intervals. Decision uncertainty arises from variations in the implementation of the calculated solution. For instance, the calculated share of compost cannot be mixed exactly using manual labor.

\section{Mathematical formulation of the problem}

In mathematical terms, we have a binary decision about whether or not to replace the plastic pot with a biodegradable pot, along with a decision about how much compost to mix with the peat. We let one potted plant be the functional unit of the problem and assume it is representative of all plants of the same species cultivated in a given nursery. For each potted plant, we must decide on

- the share of compost $\gamma \in[0,1]$ used to replace peat,

- which type of pot to use. This decision is modeled by introducing the pot variable $\beta \in\{0,1\}$, where $\beta=\left\{\begin{array}{ll}1 & \text { if a biodegradable pot chosen } \\ 0 & \text { if a plastic pot chosen }\end{array}\right.$.

Using the two decision variables $\gamma$ and $\beta$, the optimization problem for a specific plant species can be written as the following biobjective minimization problem with two objective functions and one constraint:

$$
\begin{aligned}
& \min \quad \operatorname{adC}(\gamma, \beta) \\
& \min G W P(\gamma, \beta) \\
& \text { s.t. } Q(\gamma, \beta) \geq 3 \\
& \gamma \in[0,1] \\
& \beta \in\{0,1\} \text {. }
\end{aligned}
$$

The first objective function represents the additional costs $(a d C)$ caused by the substitution, which are to be minimized. The second objective function represents the global warming potential $(G W P)$, which is also to be minimized. In the constraint, plants that fail to meet the aesthetic requirements for sale are excluded.

\section{Input data for the case study: one potted plant}

The decision problem is elucidated here by means of a plant nursery in Pistoia, Italy. Input data is collected for a potted plant of the species Photinia $x$ fraseri, which is cultivated for 1 year in a $5 \mathrm{dm}^{3}$ pot. This serves as our reference unit for the LCA calculations. Photinias are very popular ornamental shrubs, grown for their decorative fruit and foliage, especially in Italy 
Pardossi et al. (2009); Chilosi et al. (2017). The two growth media considered are a generic Baltic sphagnum peat and compost made of olive mill waste; the two planter pots considered are non-biodegradable high-density polyethylene and biodegradable polylactid acid.

The environmental emissions, costs, and life cycles of these materials are treated as follows. We use GWP, one of the most relevant indicators in LCA Guinée et al. (2002). It is expressed as carbon dioxide equivalent emissions $\left(\mathrm{kg} \mathrm{CO}_{2}\right.$-eq) and has a time horizon of 100 years. It is widely acknowledged, however, that LCA contains many sources of uncertainty, including data inaccuracy and gaps, unrepresentative data, model uncertainty, uncertainty due to choices, spatial and temporal variability, variability between sources and objects, epistemological uncertainty, measurement errors, and estimation uncertainty Björklund (2002). Therefore, we use minimum and maximum values for GWP. The prices for peat, compost, plastic pot, and biodegradable pot are also given as intervals (see Table 1).

The GWP for peat and the additional costs associated with substitutions pertain to the life cycle stages from peatland extraction to transportation to the plant nursery. GWP emissions from peat handling are negligible and can be neglected. The GWP emissions resulting from peat extraction vary from study to study Couwenberg (2011). We take as our lower value the $550 \mathrm{~kg} \mathrm{CO}$-eq per ton of material reported in Brinkmann et al. (2004) and as our upper value the $1197 \mathrm{~kg} \mathrm{CO}_{2}$-eq per ton of material reported in Kranert et al. (2007). The costs of peat are those of commercial peat (Gramoflor-Containersubstrat ${ }^{\circledR}$ ) produced in Germany.

The compost used in this study is produced from by-products of an olive-oil chain Altieri et al. (2011): de-stoned olive mill waste, wastewater, olive leaves, and twigs. These are mixed in a feed-mixing lorry, together with waste wool and straw. The resulting mixture is packaged in net sacks and stored outdoors for three months to permit static-pile composting. The compost is then allowed to mature to a final product - a process involving periodic manual turning. It can then be transported to the plant nursery and used to prepare the growth substrate. These additional steps lead to additional costs for logistics and production. The life cycle stages from the production of the by-product, the processing, and the use as growth medium for the substrate of plants are taken into account.

Plastic pots made of high-density polyethylene are commonly used in horticulture in Italy Lazzerini et al. (2016). The typical upper and lower GWP values for this material are reported by Yates and Barlow (2013). The minimum and maximum costs of plastic pots are taken from three commercial products on the Italian market.

The biodegradable pot used in this study is made of polylactic acid (PLA), which is produced from the hydrolysis of polysaccharides as starches or lignocellulosic materials Gross and Kalra (2002). The LCA of PLA includes the life cycle stages of of corn production and transport to corn wet mill, destrose and lactic acid production, lactide production, polylactide production, and injection molding. LCA studies by Hottle et al. (2013); Yates and Barlow (2013) yield the lower and upper GPW values for PLA. It is assumed that PLA pots are 30\% more expensive than plastic pots, as reported by Bastioli (2001); Riggi et al. (2011).

The fluctuations in costs and emissions during the processing of raw materials (e.g., electricity consumption) lead to parameter uncertainty. Minimum, maximum, and average values for GWP and costs are shown in Table 1. 
Table 1: (Minimum $\mid$ maximum|average) values of the problem parameters costs $(\underline{c}|\bar{c}| \widehat{c})$ and emissions $(\underline{e m}|\overline{e m}| \widehat{e m})$.

\begin{tabular}{|c|c|c|c|c|c|c|}
\hline Material & $\begin{array}{l}\text { GWP }(\mathrm{em}) \\
\left(\mathrm{kg} \mathrm{CO}_{2} \text {-eq }\right) \\
\min (\underline{\mathrm{em}})\end{array}$ & $\max (\overline{e m})$ & $\operatorname{avg}(\widehat{e m})$ & $\begin{array}{l}\text { Costs }(c) \\
(\text { Euro) } \\
\min (\underline{c})\end{array}$ & $\max (\bar{c})$ & $\operatorname{avg}(\widehat{c})$ \\
\hline peat & 0.498 & 1.083 & 0.79 & 0.16 & 0.24 & 0.20 \\
\hline compost & 0.205 & 0.342 & 0.27 & 0.03 & 0.05 & 0.03 \\
\hline plastic pot & 0.384 & 0.576 & 0.48 & 0.86 & 1.30 & 1.08 \\
\hline biodegradable pot & 0.217 & 0.403 & 0.31 & 0.98 & 1.82 & 1.40 \\
\hline
\end{tabular}

\section{The deterministic problem}

The problem analyzed in this paper has two objective functions, one economic (the additional costs of the substitutions) and one ecological (the global warming potential). Since the two objectives have incommensurable measurement units, the problem is modeled as a biobjective problem. In this section, we describe the two objectives in detail, before an analytical description of the deterministic efficient solutions is stated that is originally developed in Krüger (2018).

\subsection{Analysis of additional costs}

The first objective, the additional costs, has two parts: the purchase costs of the input materials (growth media and pot) and the selling price of the potted plant. All other costs can be neglected, since they are independent of the decision problem being considered here.

The following cost function costs: $[0,1] \times\{0,1\} \rightarrow \mathbb{R}$ is obtained as a part of the additional costs function,

$$
\begin{aligned}
\operatorname{costs}(\gamma, \beta) & =c^{\text {peat }} \cdot(1-\gamma)+c^{\text {comp }} \cdot \gamma+c^{\text {plas }} \cdot(1-\beta)+c^{\text {bio }} \cdot \beta \\
& =c^{\text {peat }}+c^{\text {plas }}+\gamma \cdot\left(c^{\text {comp }}-c^{\text {peat }}\right)+\beta \cdot\left(c^{\text {bio }}-c^{\text {plas }}\right),
\end{aligned}
$$

where the parameters $c^{\text {peat }}, c^{\text {comp }}, c^{\text {plas }}, c^{\text {bio }}$ denote the costs of one functional unit of peat, one functional unit of compost, a plastic pot, and a biodegradable pot, respectively.

The selling price of a potted plant depends on its quality, and each potted plant is assigned a specific quality level between 1 and 5. The lower the quality level, the lower the selling price of the potted plant. To determine the quality level of a potted plant, a qualitative agronomic and aesthetic evaluation by experts is used. Replacing peat with compost changes the agronomic quality of the plant, see Papafotiou et al. (2005). For the Photinia used in our case study, it is assumed that the quality function $Q$ assigns a quality level to each decision, that is,

$$
Q:[0,1] \times\{0,1\} \rightarrow\{1, \ldots, 5\} .
$$

The quality function $Q$ is piecewise constant for each $\beta \in\{0,1\}$, where the bounds of the constant parts of the quality function are

$$
0=t_{0}^{\beta}<t_{1}^{\beta}<t_{2}^{\beta}<\cdots<t_{8}^{\beta}=1 .
$$




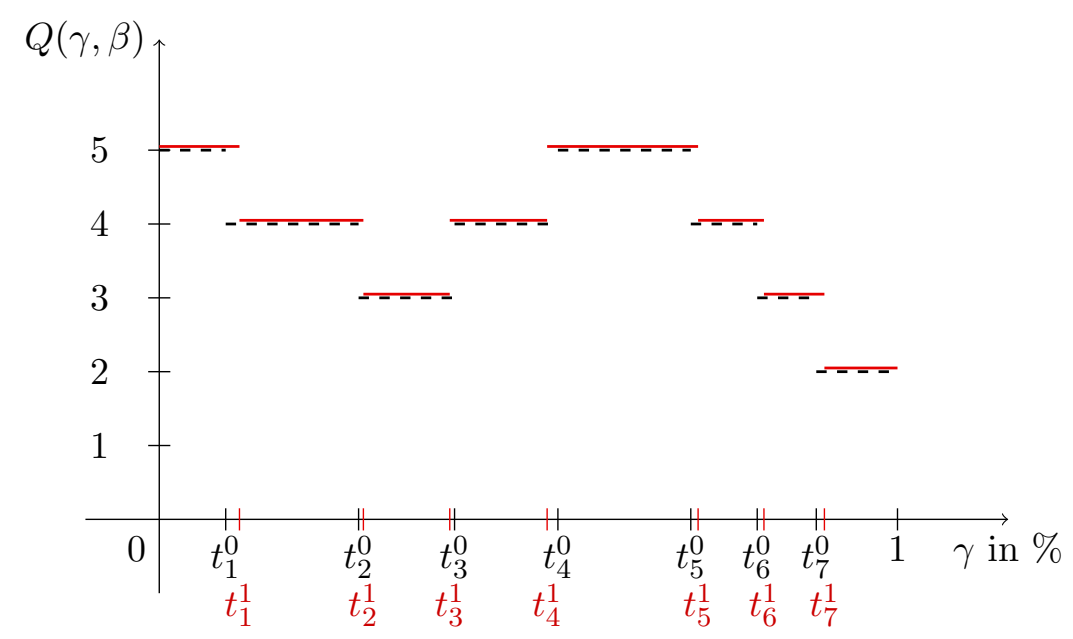

Figure 1: Quality function for the species Photinia $x$ Fraseri. Dashed black lines indicate a plastic pot $(\beta=0)$ and straight lines indicate a biodegradable pot $(\beta=1)$.

The specific quality function for a fixed pot $\beta \in\{0,1\}$ for the species Photinia with respect to one potted plant is

$$
Q(\gamma, \beta):= \begin{cases}5, & \text { if } \gamma \in\left[t_{0}^{\beta}, t_{1}^{\beta}\right] \cup\left[t_{4}^{\beta}, t_{5}^{\beta}\right] \\ 4, & \text { if } \gamma \in\left(t_{1}^{\beta}, t_{2}^{\beta}\right] \cup\left[t_{3}^{\beta}, t_{4}^{\beta}\right) \cup\left(t_{5}^{\beta}, t_{6}^{\beta}\right] \\ 3, & \text { if } \gamma \in\left(t_{2}^{\beta}, t_{3}^{\beta}\right) \cup\left(t_{6}^{\beta}, t_{7}^{\beta}\right] \\ \leq 2, & \text { if } \gamma \in\left(t_{7}^{\beta}, 1\right] .\end{cases}
$$

Figure 1 shows the dependence of the quality on the share of compost for Photinia.

Dashed and straight lines in Figure 1 demonstrate how the quality function $Q$ depends on the choice of planter pots. It is assumed that plants raised in biodegradable pots (straight lines) are of better or equal quality than plants raised in plastic pots (dashed lines).

Following the qualitative evaluation, each quality level can be associated to the selling price. It is assumed that a given number of quality levels can be attained, but that only plants with high enough levels are suitable for selling. For our case study, this minimum quality level is 3 . The relationship between the quality and the price (in [€]) of a potted plant depends on the species and is defined by the function price, which assigns a price to the quality levels.

The first objective $a d C$ is then obtained by combining the selling price and the costs

$$
\operatorname{adC}(\gamma, \beta)=\operatorname{costs}(\gamma, \beta)-\operatorname{price}(Q(\gamma, \beta)) .
$$

\subsection{Analysis of global warming potential}

The second objective function GWP considers the decision-relevant emissions of a potted plant. For each feasible share of compost $\gamma$, the GWP is the linear combination of the corresponding values for $100 \%$ peat and $100 \%$ compost:

$$
\begin{aligned}
G W P(\gamma, \beta) & =e m^{\text {peat }} \cdot(1-\gamma)+e m^{\text {comp }} \cdot \gamma+e m^{\text {plas }} \cdot(1-\beta)+e m^{\text {bio }} \cdot \beta \\
& =e m^{\text {peat }}+e m^{\text {plas }}+\gamma \cdot\left(e m^{\text {comp }}-e m^{\text {peat }}\right)+\beta \cdot\left(e m^{\text {bio }}-e m^{\text {plas }}\right),
\end{aligned}
$$


where $\mathrm{em}^{\text {peat }}, \mathrm{em}^{\mathrm{comp}}, \mathrm{em}^{\text {plas }}, \mathrm{em}^{\text {bio }}$ denote the GWP of one functional unit of peat or compost and the GWP of a plastic or biodegradable pot.

\subsection{Deterministic efficient solutions to the problem}

Since our problem has two objectives, we need a concept of optimality for multiobjective problems. We subsequently use the classical optimality concept for this case, which is the well-known Pareto efficiency, see Ehrgott (2005); Miettinen (2012). In the concept of Pareto efficiency, a point $y$ dominates a point $z$ if $y$ is less than or equal to $z$ in every component, and strictly less than $z$ in at least one component. A solution $x^{*}$ is called efficient, if there is no other feasible solution $x$ whose outcome in the objective space dominates the outcome of $x^{*}$. Hence, an efficient solution is one for which there exists no other solution that is better or equal in terms of additional costs and GWP and strictly better in at least one of them.

We now describe an approach for finding the efficient solutions. The first step is to split the problem $\mathcal{P}$ into two parts, that is, to solve the problem separately for $\beta=0$ and for $\beta=1$. The efficient solutions can then be determined by a pairwise comparison of the efficient solutions for both parts. In the remainder of this section, the pot variable $\beta \in\{0,1\}$ is considered as fixed, and the efficient solutions are determined for each $\beta$. The set of feasible solutions with respect to the fixed pot variable $\beta$ is denoted as $\mathfrak{F}(\beta)$ and for the case study we have $\mathfrak{F}(\beta)=\left[0, t_{7}^{\beta}\right]$. The quality level of the rightmost point of $\mathfrak{F}(\beta)$ is given as $\nu_{\min }=3$ according to $(1)$.

To find efficient solutions, we consider each quality level separately. This fixes the selling price. Since the additional costs and GWP are both minimized for a given quality level if we choose as much compost as possible (cf. Table 1), we need only consider the rightmost points (Figure 2). However, we must still determine whether they are efficient or not.

Formally, the set of efficient solutions to the deterministic problem for the fixed pot variable $\beta$ is given as

$$
\mathcal{D}(\beta):=\bigcup_{j=\nu_{\min }}^{5}\{\max \{\gamma \in \mathfrak{F}(\beta) \mid Q(\gamma, \beta) \geq j\}\} .
$$

This result confirms the intuitive approach of taking the maximum share of compost in a plant nursery: for each quality level, all higher and equal quality levels are considered, and the maximum share of compost thereof is chosen. Therefore, the set of deterministic efficient solutions is easily computable.

In this section, we have described the cost function for purchasing growth media and pot, the quality function for identifying suitable quality levels for selling, the additional costs function, the environmental function, and the deterministic approach to solving the problem. Since this approach is not suitable for the decision problem when it includes uncertainty, however, we present an appropriate approach in the following section. 


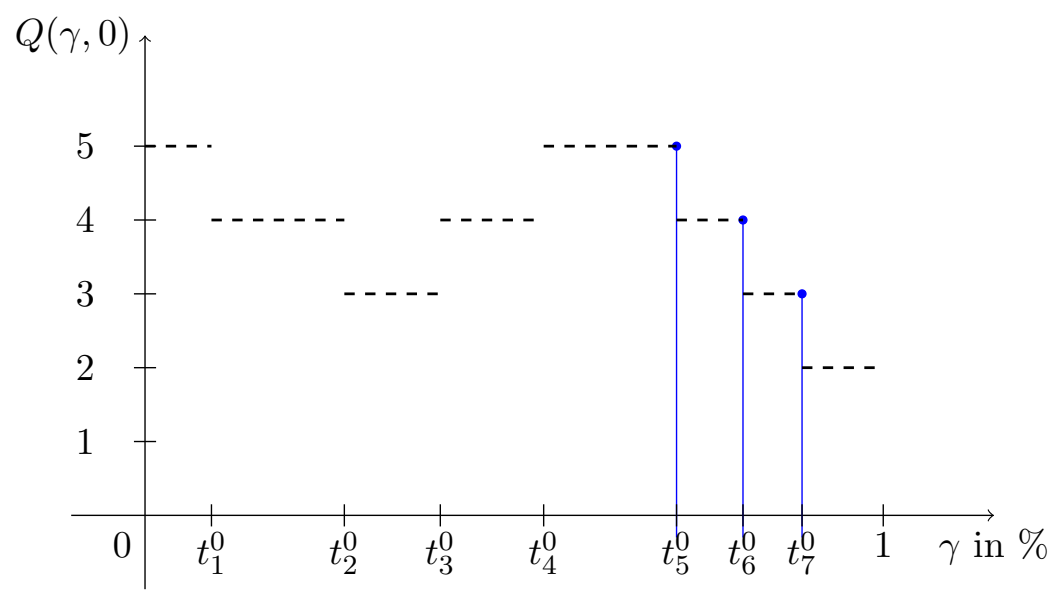

Figure 2: Illustration of Equation (4). The dots mark the quality levels of the efficient solutions for a plastic pot $t_{5}^{0}, t_{6}^{0}, t_{5}^{0}$.

\section{Uncertainty in the problem}

In our case study, costs and emissions are expressed as ranges or intervals, rather than as exact numbers. This creates parameter uncertainty. And our solutions - at least those concerning the share of compost in the growth medium - cannot be put into practice exactly, but are burdened with deviations. This creates decision uncertainty. In the literature, these two types of uncertainty are also referred to as data uncertainty and implementation error Ben-Tal et al. (2009); Ben-Tal and Nemirovski (2002); Bertsimas et al. (2007).

There are various ways to address these two types of uncertainty in biobjective problems such as $\mathcal{P}$. Robust optimization is especially suitable for problems in which the uncertainty set (i.e., the set of all scenarios that may occur) is known, and where knowledge about a probability distribution on the uncertainty set is not given Bertsimas et al. (2011).

\subsection{Parameter uncertainty}

Various robustness concepts for parameter uncertainty in bi-objective or multiobjective problems have been introduced in the literature. In each case, it is assumed that there is a so-called uncertainty set containing all possible values of the data. Each element of the uncertainty set is called a scenario. In the decision problem $\mathcal{P}$, each parameter $c^{\text {peat }}, c^{\text {comp }}, c^{\text {plas }}, c^{\text {bio }}$ and $\mathrm{em}^{\text {peat }}, \mathrm{em}^{\mathrm{comp}}, \mathrm{em}^{\text {plas }}, \mathrm{em}^{\text {bio }}$ falls within an interval, and the intervals are independent of each other. For example, $c^{\text {peat }} \in\left[\underline{c}^{\text {peat }}, \bar{c}^{\text {peat }}\right]$, see Table 1 . The worst case is given by

$$
\bar{c}^{\text {peat }}, \bar{c}^{\text {comp }}, \bar{c}^{\text {plas }}, \ldots, \overline{e m}^{\text {plas }}, \overline{e m}^{\text {bio }} \text {. }
$$

Here, all parameters are at the upper bounds of their intervals. This is a special situation in robust optimization in which we can find a robust solution by considering these worst-case values. That is, we replace $c^{\text {peat }}$ by $\bar{c}^{\text {peat }}, \ldots, c^{b i o}$ by $\bar{c}^{\text {bio }}$ in the first objective function $a d C$, and we replace $e m^{\text {peat }}$ by $\overline{e m}^{\text {peat }}, \ldots, \mathrm{em}^{\text {bio }}$ by $\overline{\mathrm{em}}^{\text {bio }}$ in the second objective function $G W P$. The parameter uncertainty is then included in the resulting problem's objective functions. 


\subsection{Decision uncertainty}

Since, for our problem, it is reasonable to assume that a worker uses exactly the chosen type of pot, the only decision uncertainty is the share of compost in the substrate. In horticulture, growth substrate is prepared for many potted plants at one time, and the mixing is done roughly in big heaps using skid-steer loaders. The maximum and minimum deviation in compost share $\gamma$ that can be expected in a single pot can be estimated by the plant nursery based on experience (e.g., $10 \%$ lower or $10 \%$ higher than intended).

For our problem, we assume that both a minimum deviation $z^{\text {min }} \leq 0$ and a maximum deviation $0 \leq z^{\max }$ are given. The actual values of $\gamma$ are expected to fall within $\left[\gamma+z^{\min }, \gamma+\right.$ $\left.z^{\max }\right]$. However, this is not correct for values $\gamma<-z^{\min }$ or $\gamma>1-z^{\max }$, since a share below $0 \%$ or above $100 \%$ is not permitted. The uncertainty when implementing a decision hence depends on the values of $\gamma$. It is defined as

$$
\begin{aligned}
& z^{\min }(\gamma):= \begin{cases}\max \left\{z^{\min },-\gamma\right\} & \text { for } \gamma \in(0,1) \\
0 & \text { for } \gamma \in\{0,1\}\end{cases} \\
& z^{\max }(\gamma):= \begin{cases}\min \left\{z^{\max }, 1-\gamma\right\} & \text { for } \gamma \in(0,1) \\
0 & \text { for } \gamma \in\{0,1\}\end{cases}
\end{aligned}
$$

to yield the deviation set $Z(\gamma)$ of $\gamma \in[0,1]$ as

$$
Z(\gamma):=\left[z^{\min }(\gamma), z^{\max }(\gamma)\right]
$$

The realized values for every $\gamma \in(0,1)$ are finally obtained in

$$
\{\gamma\}+Z(\gamma)=\left[\max \left\{\gamma+z^{\min }, 0\right\}, \min \left\{\gamma+z^{\max }, 1\right\}\right] .
$$

The cases in which no mixing is needed $(\gamma=0$ and $\gamma=1)$ can be realized exactly.

In the deterministic case, every solution $(\gamma, \beta)$ is realized exactly. In in case of decision uncertainty, however, there are many possible outcomes of the solution $(\gamma, \beta)$, one for each realization $\gamma+z$ with $z \in Z(\gamma)$. The plant nursery would like to avoid realizations in intervals that lead to low quality. That is, it would like all possible realizations of a share $\gamma$ to lead to sellable plants. Therefore, we only consider solutions $\gamma$, for which the plant can be sold in every realization. The set of solutions $\gamma$ with this property is called the robust feasible set, and it is denoted as $\mathcal{X}^{\mathrm{RC}}$. This concept of robust feasibility was introduced by Eichfelder et al. (2017); Ben-Tal et al. (2009).

The next question is this: which of the solutions are good choices? One might choose the solutions from Section 4.3 for the deterministic case. However, these might not be the best choice if $\gamma$ is not implemented as planned, since one might obtain a substrate mixture that drops into a lower quality level. Thus, the aim is to search for solutions that are robust against these deviations. For every solution $\gamma$, the set of its realizations must be considered. In other words, when assessing outcomes, one must compare sets instead of points. To accomplish the complex task of comparing two possible solutions, we use the definition of robust efficiency provided by (Eichfelder et al., 2017, Eq.(3)). Here, two solutions are compared by comparing all their possible realizations with each other in the following way. A solution $\gamma^{*}$ dominates a solution $\gamma$ if, for every scenario $z \in Z(\gamma)$, there exists a (perhaps different) scenario $z^{*} \in Z\left(\gamma^{*}\right)$ such that the outcome of $\gamma+z$ is dominated by the outcome of $\gamma^{*}+z^{*}$. The set of the robust efficient solutions is denoted by $\mathcal{E}$, and the set of robust efficient solutions for a fixed pot $\beta$ is $\mathcal{E}(\beta)$. 


\subsection{Robust solutions to the problem}

To determine the robust efficient solutions to the problem with decision uncertainty, the approach of Krüger (2018) is followed using their analytical description of the solutions. The problem is split into two parts and solved separately for $\beta=0$ (plastic pot) and for $\beta=1$ (biodegradable pot). The set of robust efficient solutions can then be derived by a pairwise comparison of the robust efficient sets for the two values of $\beta$. As shown in Krüger (2018), the number of robust solutions for each fixed pot variable $\beta \in\{0,1\}$ is small. Hence, only a small number of pairwise comparisons is necessary.

In the remainder of this section, we therefore assume that the pot variable $\beta \in\{0,1\}$ is fixed and determine the robust solutions for each $\beta$. The robust feasible set for the fixed variable $\beta$ is denoted as $\mathcal{X}_{\beta}^{\mathrm{RC}}$. To illustrate $\mathcal{X}_{\beta}^{\mathrm{RC}}$, we consider the quality function in Figure 1. The dotted line at $t_{7}^{0}$ indicates that, in a plastic pot $(\beta=0)$, every share of compost greater than $t_{7}^{0}$ yields a plant not suitable for sale. When dealing with decision uncertainty, if a plant nursery assumes that the realized values of $\gamma$ differ from their computed values (e.g., by at most $\pm 10 \%$,), then a safety margin is required between the robust feasible solutions and those constant intervals of $Q$ where the quality drops below 3 . In our case study, the safety margin corresponds to the maximum deviation from the computed value. From the point of view of the plant nursery, the safety margin is the maximum hypothetical deviation for which the quality level of a potted plant does not deviate from the expected level. For the quality function in Figure 1, this can easily be achieved by subtracting $10 \%$ from the maximum feasible solution $t_{7}^{\beta}$, that is, by taking $t_{7}^{\beta}-0.1$ as the maximum solution yielding a plant that can always be sold.

The following method based on Krüger (2018) can be used to determine the robust efficient set $\mathcal{E}(\beta)$ :

(i) take all interval bounds of the quality function and subtract the maximum deviation $z^{\max }$

(ii) check whether these points are robust feasible and keep only the robust feasible points, neglecting the rest. The resulting set is then called $S(\beta)$;

(iii) choose the biggest element of the set $S(\beta)$ and determine the minimum quality that the realizations of this element can have as $j_{\text {max }}^{\beta}$;

(iv) for each quality level $j$ from $j_{\max }^{\beta}$ until the maximum quality (i.e., 5 ), proceed as follows: for every element of the set $S(\beta)$, determine the minimum quality of its realizations. If this quality is lower than $j$, delete that element from the set. Then, choose the biggest element of the remaining set and add it to the set $\mathcal{W}(\beta)$.

This procedure yields the set $\mathcal{W}(\beta)$, in which every element is robust. One must now check whether there are further robust solutions. To determine the whole set of robust solutions, only two cases must be distinguished:

(a) if all elements of the set $\mathcal{W}(\beta)$ are larger than the minimum deviation $\left|z^{\text {min }}\right|$, then the set of robust solutions is exactly $\mathcal{W}(\beta)$;

(b) if the smallest element of $\mathcal{W}(\beta)$ is smaller than $\left|z^{\text {min }}\right|$, then the set of robust solutions is not only $\mathcal{W}(\beta)$, but also includes a whole interval of robust solutions between 0 and the maximum element of the set $\mathcal{W}(\beta)$ that is smaller than $\left|z^{\text {min }}\right|$. 
Since there can never be less than $0 \%$ compost, the realization sets of the solutions near zero are nested within each other. This makes their outcomes hard to distinguish, thus leading to the whole interval of robust solutions. This is why there is an interval of robust solutions if any element of $\mathcal{W}(\beta)$ is larger than the minimal deviation $\left|z^{\text {min }}\right|$.

As described in this section, the robust solutions can be determined directly by evaluating the set $\mathcal{W}(\beta)$, which itself can be calculated with low computational effort from the finite set of interval bounds of $Q$. The suggested steps for computing the set $\mathcal{W}(\beta)$ are similar to those for the deterministic problem. The main differences are that a safety margin is considered in the robust feasible set and that the finite candidate set is evaluated with respect to the minimal quality of its element's realizations.

\section{Case study for the species Photinia}

The model is applied to the real-world case study using the solution techniques for the deterministic and uncertain cases. The deterministic and robust solutions are determined and compared from the practical perspective of a plant nursery.

\subsection{Deterministic efficient solutions}

We now present a detailed study by combining the results of Section 4 with the data in Table 1 and Table 2, which is illustrated in Figure 1.

Table 2: Boundaries of the constant parts of the quality function $Q$ in [\%].

\begin{tabular}{c|c|c|c|c|c|c|c|c|c} 
& $t_{0}^{\beta}$ & $t_{1}^{\beta}$ & $t_{2}^{\beta}$ & $t_{3}^{\beta}$ & $t_{4}^{\beta}$ & $t_{5}^{\beta}$ & $t_{6}^{\beta}$ & $t_{7}^{\beta}$ & $t_{8}^{\beta}$ \\
\hline$\beta=0$ & 0 & 9 & 27 & 40 & 54 & 72 & 81 & 89 & 100 \\
\hline$\beta=1$ & 0 & 10.9 & 27.7 & 39.4 & 52.5 & 73 & 81.9 & 90.1 & 100 \\
\hline
\end{tabular}

The price function of a Photinia is displayed in Table 3 . Here, a potted plant grown in peat can always be sold for $6,50 €$ (quality level 5, without substitution). When using compost, additional selling costs (due to the lower prices) are assumed between $2,17 €$ (quality level 4) and $4,34 €$ (quality level 3 ).

Table 3: Price function of a potted Photinia x Fraseri

\begin{tabular}{c|c}
\hline Quality $Q(\cdot)$ & Price $\operatorname{price}(Q(\cdot))$ \\
\hline 5 & $6.50 €$ \\
\hline 4 & $4.33 €$ \\
\hline 3 & $2.16 €$ \\
\hline
\end{tabular}

Applying Equation (4), the following sets of deterministic efficient solutions are obtained for the pot variable values $\beta \in\{0,1\}$, 


$$
\mathcal{D}(0)=\left\{t_{5}^{0}, t_{6}^{0}, t_{7}^{0}\right\} \quad \text { and } \quad \mathcal{D}(1)=\left\{t_{5}^{1}, t_{6}^{1}, t_{7}^{1}\right\}
$$

The outcomes of the efficient solution sets $\mathcal{D}(0)$ and $\mathcal{D}(1)$ are displayed in Figure 3.

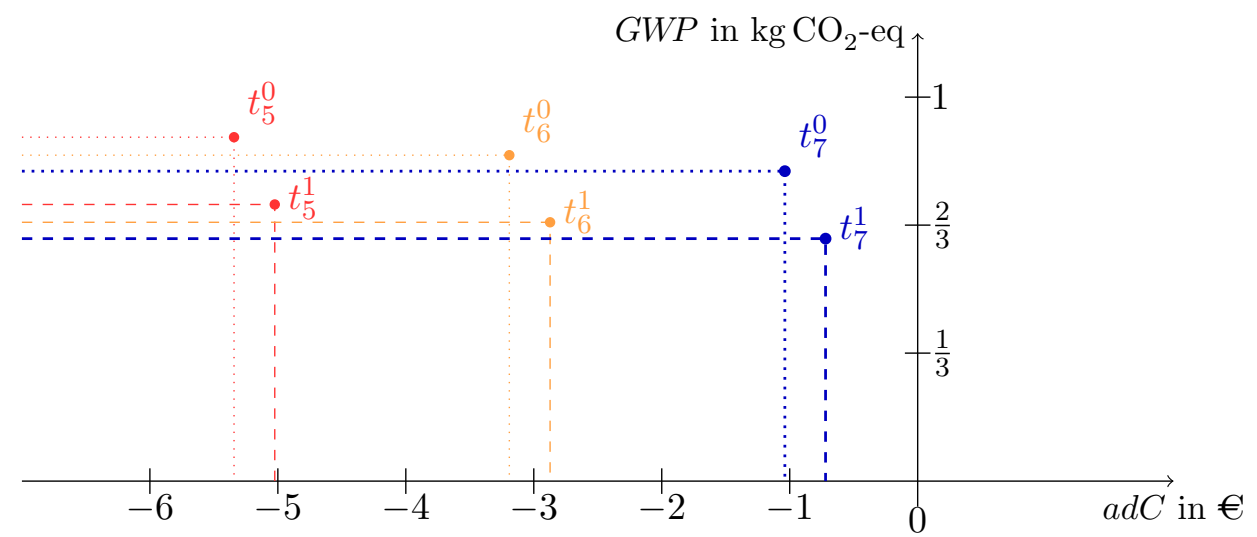

Figure 3: Deterministic efficient solutions for both types of planter pots: $t_{5}^{0}, t_{6}^{0}, t_{7}^{0}$ are the efficient solutions with plastic pots, whereas $t_{5}^{1}, t_{6}^{1}, t_{7}^{1}$ are the efficient solutions with biodegradable pots. The lines represent the Pareto order with which the solutions are compared. Dotted lines correspond to plastic pots $\mathcal{D}(0)$, and dashed lines correspond to biodegradable pots, i.e., $\mathcal{D}(1)$.

As shown in Figure 3, the solution $t_{6}^{0} \in \mathcal{D}(0)$ is dominated by $t_{5}^{1} \in \mathcal{D}(1)$ and the solution $t_{7}^{0} \in \mathcal{D}(0)$ is worse than each of the solutions $t_{5}^{1}, t_{6}^{1} \in \mathcal{D}(1)$ in both objectives, adC and GWP. The set of (deterministic) efficient solutions to the problem is hence given as

$$
\mathcal{D}=\left\{\left(t_{5}^{0}, 0\right),\left(t_{5}^{1}, 1\right),\left(t_{6}^{1}, 1\right),\left(t_{7}^{1}, 1\right)\right\} .
$$

These solutions are illustrated in Figure 4. One sees immediately that there are three solutions with a biodegradable pot and only one with a plastic pot. Hence, the choice of a bio-pot goes well with replacing peat by compost, even though a biodegradable pot is more expensive. Although the most profitable solution $\left(t_{5}^{0}, 0\right)$ involves a plastic pot, there are also three efficient solutions involving a biodegradable pot. Moreover, compared to $\left(t_{5}^{0}, 0\right)$, choosing $\left(t_{5}^{1}, 1\right)$ yields only slightly more $a d C$ while improving $G W P$ significantly. The solution $\left(t_{5}^{1}, 1\right)$ is the best solution for a plant nursery when both objectives are equally weighted. If the economic objective is weighted significantly higher, then $\left(t_{5}^{0}, 0\right)$ should be chosen. Conversely, a high preference for the ecological objective leads to either $\left(t_{6}^{1}, 1\right)$ or $\left(t_{7}^{1}, 1\right)$.

\subsection{Robust solutions}

In this section, we take uncertainties into account. In our problem, the data for costs and emissions is considered uncertain, and the exact share of compost in the growth media is unknown. To account for uncertainty in the cost $(c)$ and emissions $(\mathrm{em})$ parameters, we assume that their values lie in the intervals given in Table 1. To account for uncertainty in the realized share of compost $\gamma$ in the growing media, we assume that it varies from its intended quantity by $\pm 10 \%$ - an estimate based on plant nursery experience. Therefore, $z^{\min }=-0.1$ and $z^{\max }=0.1$. 


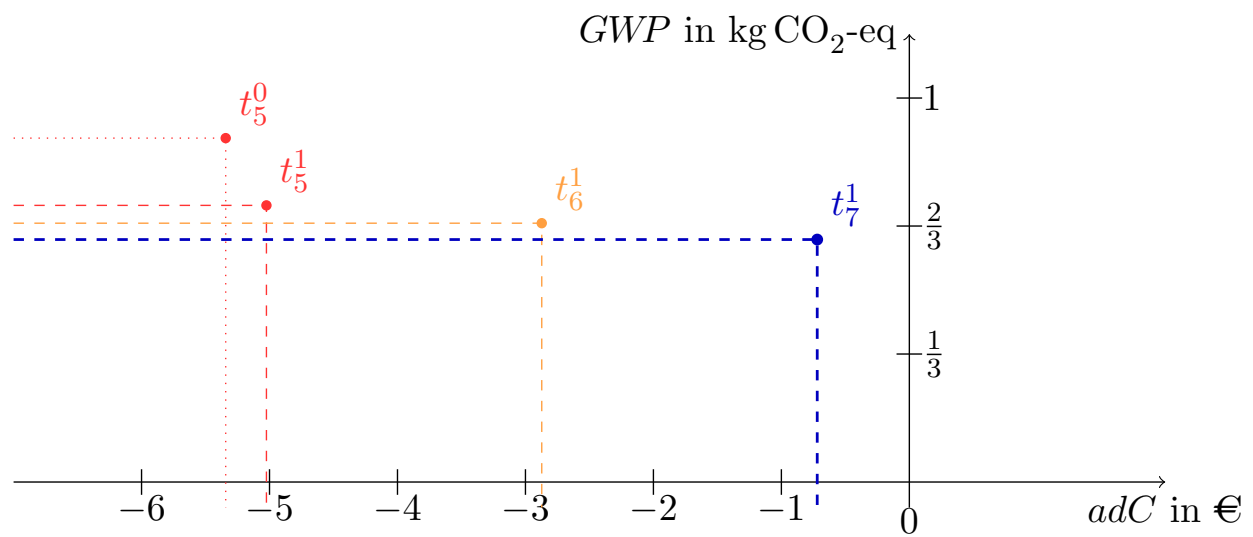

Figure 4: Deterministic efficient solutions

Using the framework from Section 5, we next determine the robust solutions to our problem for the species Photinia.

We follow the procedure from Section 5.2 to determine the set $\mathcal{W}(\beta)$ for each choice of the pot variable $\beta \in\{0,1\}$. Toward this end, we first shift the set of boundaries of the constant intervals of the quality function by $z^{\max }$ (see Table 2). This yields

$$
\left\{\left(t_{1}^{\beta}-z^{\max }\right),\left(t_{2}^{\beta}-z^{\max }\right), \ldots,\left(t_{7}^{\beta}-z^{\max }\right),\left(1-z^{\max }\right)\right\} \cup\{0,1\}
$$

This set is then reduced to the elements that are robust feasible for the problem with uncertainty, and the result is

$$
\begin{aligned}
S(0) & =\left\{0,\left(t_{2}^{0}-z^{\max }\right), \ldots,\left(t_{7}^{0}-z^{\max }\right)\right\} \\
S(1) & =\left\{0,\left(t_{1}^{1}-z^{\max }\right),\left(t_{2}^{1}-z^{\max }\right), \ldots,\left(t_{7}^{1}-z^{\max }\right)\right\} .
\end{aligned}
$$

The maximum element of each of these sets is

$$
\begin{aligned}
& t_{7}^{0}-z^{\max }=0.79 \\
& t_{7}^{1}-z^{\max }=0.80,
\end{aligned}
$$

the minimum quality that both solutions can attain is given by

$$
j_{\max }^{0}=\min _{z \in[-0.1,0.1]} Q\left(t_{7}^{0}-z^{\max }+z, 0\right)=3=\min _{z \in[-0.1,0.1]} Q\left(t_{7}^{1}-z^{\max }+z, 1\right)=j_{\text {max }}^{1},
$$

where the set $[-0.1,0.1]$ reflects the fact that every solution is assumed to vary from its intended value by $\pm 10 \%$. Note that the solution technique does not depend on $z^{\max }$ (chosen here to be 0.1$)$, and it can be adapted to any choice of $z^{\max }$.

Calculating the minimum quality $\min _{z \in[-0.1,0.1]} Q(s+z, \beta)$ for each $s \in S(\beta)$ and $\beta \in$ $\{0,1\}$, and determining the largest element of $S(\beta)$ for each quality level $\left\{j_{\max }^{\beta}, \ldots, 5\right\}=$ $\{3,4,5\}$, we obtain

$$
\begin{aligned}
& \mathcal{W}(0)=\left\{0,\left(t_{6}^{0}-z^{\max }\right),\left(t_{7}^{0}-z^{\max }\right)\right\} \\
& \mathcal{W}(1)=\left\{\left(t_{5}^{1}-z^{\max }\right),\left(t_{6}^{1}-z^{\max }\right),\left(t_{7}^{1}-z^{\max }\right)\right\}
\end{aligned}
$$


For both cases, a plastic pot $(\beta=0)$ and a biodegradable pot $(\beta=1)$, the first case $(a)$ in Section 5.3 is applied, and the set of robust solutions is determined as

$$
\begin{aligned}
& \mathcal{E}(0)=\mathcal{W}(0)=\{0,0.71,0.79\} \\
& \mathcal{E}(1)=\mathcal{W}(1)=\{0.63,0.72,0.80\}
\end{aligned}
$$

Figure 5 shows the outcomes of the robust solutions for each choice of pot. The outcome of a solution is a set, rather than a point, since each solution is considered to be realized within a range, rather than exactly. In accordance with the concept of dominance in robust multiobjective optimization, the outcomes of solutions may be compared by subtracting the cone that is nonnegative orthant from each point in the outcome set. A solution $\left(\gamma^{\prime}, \beta^{\prime}\right)$ is then dominated by a solution $(\bar{\gamma}, \bar{\beta})$ if and only if the outcome set of $(\bar{\gamma}, \bar{\beta})$ is contained within the outcome set of $\left(\gamma^{\prime}, \beta^{\prime}\right)$ minus the nonnegative orthant, see Eichfelder et al. (2017); Ehrgott et al. (2014). As shown in Figure 5, the solution $\left(t_{6}^{0}-z^{\max }\right) \in \mathcal{E}(0)$ is dominated

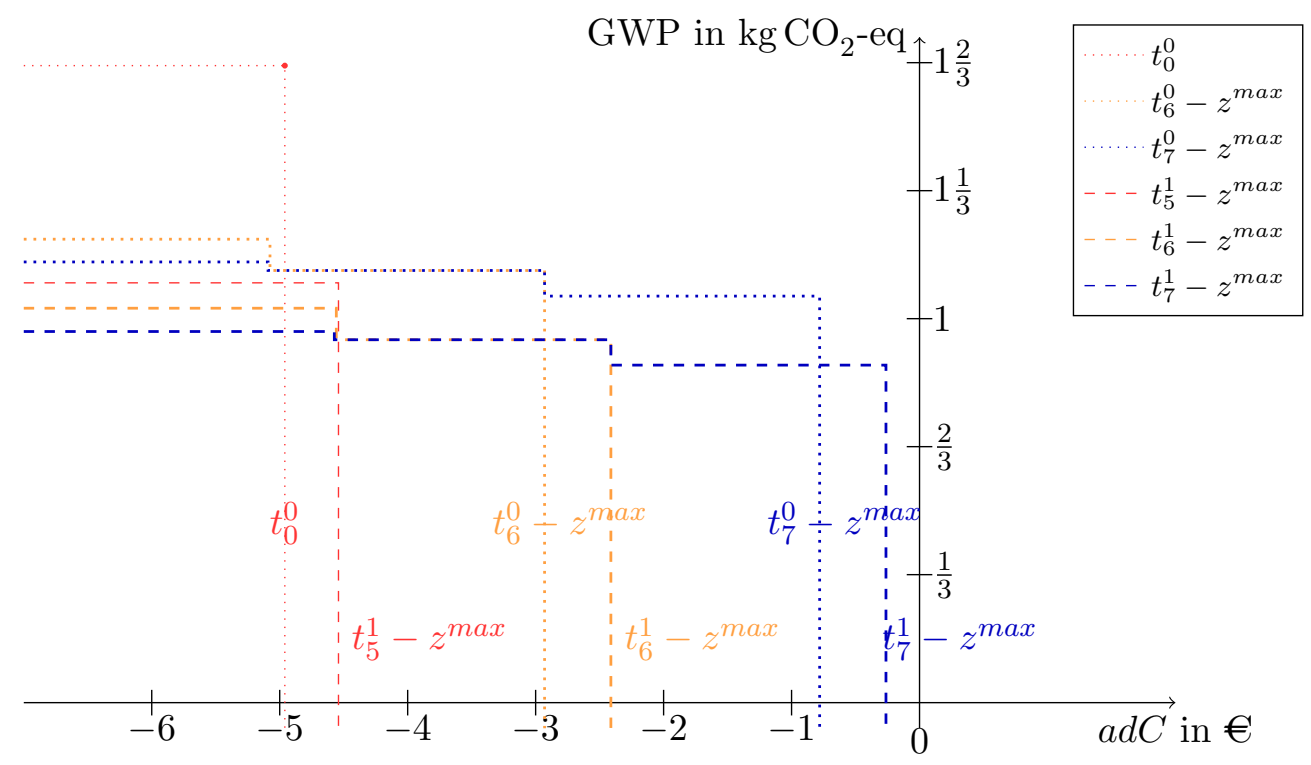

Figure 5: Outcome sets of the robust solutions for each type of planter pot. Dotted lines correspond to plastic pots $(\mathcal{E}(0))$ and dashed lines correspond to bio pots $(\mathcal{E}(1))$.

by $\left(t_{5}^{1}-z^{\max }\right) \in \mathcal{E}(1)$ and the solution $\left(t_{7}^{0}-z^{\max }\right) \in \mathcal{E}(0)$ is dominated by the solutions $\left(t_{5}^{1}-z^{\max }\right),\left(t_{6}^{1}-z^{\max }\right) \in \mathcal{E}(1)$, a result that can also be verified by direct calculation. The remaining solutions in Figure 5 do not dominate each other and are hence robust. The set of robust solutions to our problem is given as

$$
\begin{aligned}
\mathcal{E} & =\left\{(0,0),\left(t_{5}^{1}-z^{\max }, 1\right),\left(t_{6}^{1}-z^{\max }, 1\right),\left(t_{7}^{1}-z^{\max }, 1\right)\right\} \\
& =\{(0,0),(0.63,1),(0.72,1),(0.80,1)\}
\end{aligned}
$$

and it is illustrated in Figure 6.

The only robust solution involving a plastic pot is the traditional one using $100 \%$ peat $\left(t_{0}^{0}, 0\right)$. In contrast, there are three robust solutions involving a biodegradable pot, denoted by the dashed lines in Figure 6. Replacing peat with compost works best if combined with 
a biodegradable pot, that is, replacing both traditional choices of growth media and pot is better than replacing only one of them. The solution $\left(t_{5}^{1}, 1\right)$ is only slightly worse with respect to the additional costs $a d C$, but it is significantly better with respect to the environmental objective. Hence, the solution $\left(t_{5}^{1}, 1\right)$ is a good alternative to $\left(t_{0}^{0}, 0\right)$.

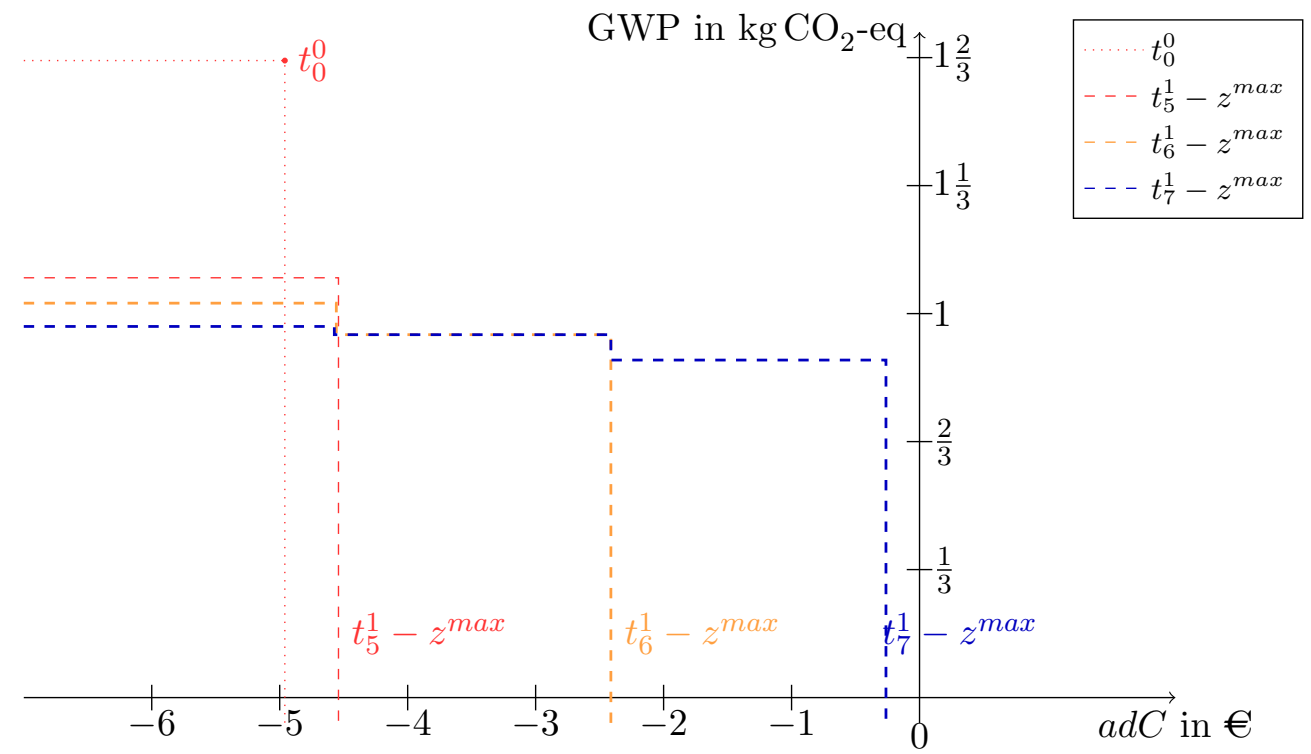

Figure 6: Outcome sets of the robust solutions. The traditional solution $t_{0}^{0}$ of $100 \%$ peat is the only robust solution involving a plastic pot and there are three robust solutions $t_{5}^{1}-$ $z^{\max }, t_{6}^{1}-z^{\max }, t_{7}^{1}-z^{\max }$ involving a biodegradable pot.

\subsection{Comparison of the deterministic and robust solutions}

We now compare the outcomes of the deterministic efficient solutions to the outcomes of the robust solutions.

Taking into account that realizations of the deterministic efficient solutions $t_{6}^{1}, t_{7}^{1} \in \mathcal{D}$ may deviate from their intended values by $\pm 10 \%$, we see, by Table 2 , that there are possible realizations of $t_{6}^{1}$ and $t_{7}^{1}$ with a quality level that is not suitable for selling. Because the choice of $t_{6}^{1}$ or $t_{7}^{1}$ might lead to plants that are not sellable, these options are not robust and are excluded from the set of robust feasible solutions.

The outcome sets of the remaining deterministic efficient solutions $t_{5}^{0}, t_{5}^{1} \in \mathcal{D}$ are illustrated by the gray lines in Figure 7 . The solution $t_{5}^{0}$ is dominated by the solutions $t_{5}^{1}-z^{\max }$ and $t_{6}^{1}-z^{\max }$, since the outcome sets of the latter are contained in the outcome set of the former minus the nonnegative orthant. Hence, the solution $t_{5}^{0}$ is not robust. The outcome of solution $t_{5}^{1}$ is partly contained in the outcome set of the robust solution $t_{7}^{1}-z^{\max }$ and the rest of this outcome set is close to the outcome set of $t_{6}^{1}-z^{\max }$. It can be verified that the solution $t_{5}^{1}$ is dominated by the solution $t_{7}^{1}-z^{\max }$ and is therefore also not robust.

One may conclude that the robust solutions in $\mathcal{E}$ are preferable to the deterministic efficient solutions in $\mathcal{D}$. Including uncertainty in the problem leads to solutions that are robust against deviations in parameters and decision variables. An important difference between the deterministic and robust solutions is the safety margin between the robust solutions and the 


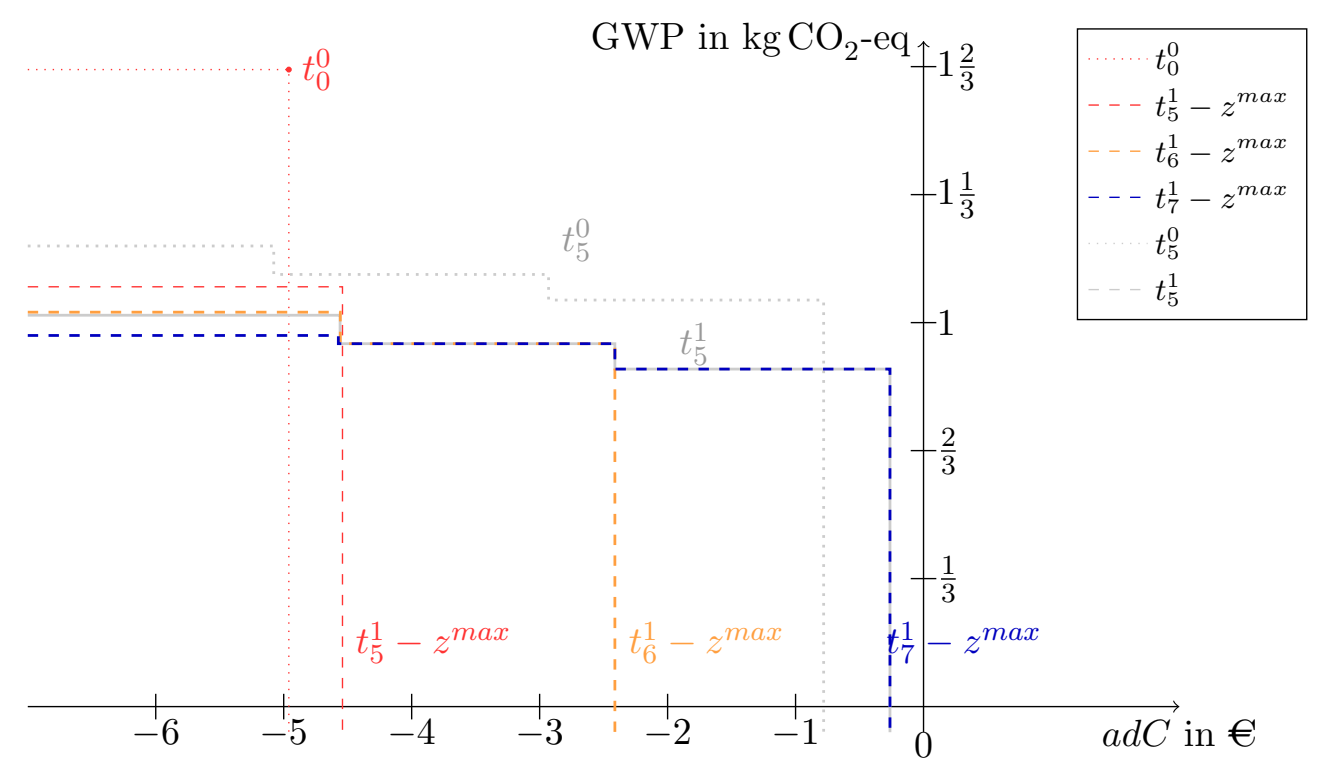

Figure 7: Comparison of the outcomes of the deterministic and robust solutions. The gray lines illustrate the outcomes of the two robust feasible deterministic efficient solution $t_{5}^{0}$ and $t_{5}^{1}$ evaluated with up to $\pm 10 \%$ more or less compost than expected. The lines reflect that the whole outcome sets of the solutions are compared by the order of the Pareto cone. The solutions for the plastic $(\beta=0)$ and biodegradable pots $(\beta=1)$ are displayed with dotted and dashed lines, respectively.

right-hand interval bounds of the quality function. A safety margin to the left-hand interval bounds is not necessary. Happily, the complete set of robust solutions can be computed with low computational effort by following the steps in Section 5 .

Another important observation is that the robust solutions can be grouped into two classes: those that retain the traditional solution, and those that replace both pots and growth media by their environmentally-friendly alternatives while retaining a safety margin to the right hand interval bound.

\section{Summary and outlook}

Peat mining alters the ecosystem and releases significant amounts of greenhouse gases into the atmosphere. Horticulture uses enormous quantities of peat as a growth medium, and its partial replacement with compost therefore greatly improves resource efficiency.

Our analysis of the horticultural production of potted plants has highlighted various aspects of uncertainty. Carbon-based growth media decompose over time, thus contributing greenhouse gases to the atmosphere. Modeling these contributions is complicated due to the varying of fractions of organic content and differences in the properties of peat and compost. Although oxidation rates could be obtained via laboratory analysis of the specific growth media components, they are often taken from the literature. Generally speaking, the quality of emissions modeling depends on the input data. In this paper, we therefore take into account measurement errors, fluctuations in conditions for preparing growth substrate, and imprecise 
horticultural data by using value intervals rather than exact values. This paper also addresses decision uncertainty in optimizing the share of input materials for horticultural growth substrate. Using a case study, we model an optimization problem with parameter and decision uncertainty and solve the model. After first finding deterministic solutions, we then determine robust solutions, while accounting for parameter and decision uncertainty.

A novel, robust multiobjective optimization approach has been applied to determine the robust solutions in presence of uncertainty. Moreover, this paper deals with quality levels in horticulture-a difficult task with uncertain inputs. Our results show that the robust solutions are preferable to the deterministic ones whenever solutions cannot be implemented exactly as planned, but only within a range of the targeted values. In our case study, the imprecise mixing practices currently used in horticultural settings bring uncertainty regarding the exact share of compost in the growth media. Thus, this share can only be estimated.

As a simplified rule of thumb derived from the results of this paper, only the rightmost point of each feasible interval of the quality function should be considered (subtracting the maximum deviation from the solutions in the pots), and for each quality level, the rightmost point of this and all higher quality levels is chosen. If all of these solutions are larger than the maximum deviation, any of them is a robust solution. However, solutions near zero are less influenced by deviations, since realizations below zero percent can not happen. In particular, the robust approach leads to solutions in which the number of plants of marketable quality is maximized. The final outcome of our study, which was not predictable before the modeling, highlights that using plastic pots in combination with compost is not an efficient solution. Instead, robust solutions involving compost also necessarily involve a biodegradable pot. A rule of thumb for robust planning is therefore to combine composted growth media with biodegradable pots.

This paper raises the question whether a scale-up of the results for a single potted plant might yield a drastic reduction in environmental impacts in the horticulture sector. What might happen if the whole Pistoia district, with its 5000 nurseries, were to modify their potting practices in favor of the more sustainable alternatives illustrated here? What would be the consequences on the carbon sink effect of the peatlands?

Future research into horticulture with uncertainty could include combining different pot capacities, varying resources (both growth medium and pot), investigating different safety margins, and solving the problem for various plant species while simultaneously taking into account that the supply of compost is ultimately limited. Including these parameters would help promote a strategic managerial perspective in horticulture, which is currently managed on the basis of tradition. And what would be the impact if the food industry (which can be affected by uncertainty in the exact recipe of ingredients) were to switch all products at the same time to more sustainable alternatives (e.g., biodegradable packaging) by modifying current supply chains? This study implies that the used approach might be applicable to other fields having a broader class of uncertain biobjective optimization problems.

As final remark, decision robust efficiency is demonstrated in this paper to be a suitable approach whenever the implemented solutions deviate from the computed solutions. Addressing further applications of decision uncertainty in multiobjective optimization is of interest in the horticultural sector and in other areas where mixing problems are relevant. 


\section{Acknowledgements}

This work was supported by DFG RTG 1703 "Resource Efficiency in Interorganizational Networks".

\section{References}

R. Altieri, A. Esposito, and T. Nair. Novel static composting method for bioremediation of olive mill waste. International Biodeterioration \& Biodegradation, 65(6):786-789, 2011.

J. Ashayeri, Van Eijs, A. G. M., and P. Nederstigt. Blending modelling in a process manufacturing: A case study. European Journal of Operational Research, 72(3):460-468, 1994.

C. Bastioli. Global status of the production of biobased packaging materials. Starch - Stärke, $53(8): 351,2001$.

A. Ben-Tal and A. Nemirovski. Robust optimization - methodology and applications. Mathematical Programming, 92(3):453-480, 2002.

A. Ben-Tal, L. El Ghaoui, and A. Nemirovski. Robust Optimization. Princeton University Press, Princeton and Oxford, 2009.

D. Bertsimas and M. Sim. The price of robustness. Operations Research, 52(1):35-53, 2004.

D. Bertsimas, O. Nohadani, and K. M. Teo. Robust optimization in electromagnetic scattering problems. Journal of Applied Physics, 101(7):074507, 2007.

D. Bertsimas, D. B. Brown, and C. Caramanis. Theory and applications of robust optimization. SIAM Review, 53(3):464-501, 2011.

A. Björklund. Survey of approaches to improve reliability in lca. The International Journal of Life Cycle Assessment, 7(2):64-72, 2002.

C. Bohle, S. Maturana, and J. Vera. A robust optimization approach to wine grape harvesting scheduling. European Journal of Operational Research, 200(1):245-252, 2010.

M. Brandenburg, K. Govindan, J. Sarkis, and S. Seuring. Quantitative models for sustainable supply chain management: Developments and directions. European Journal of Operational Research, 233(2):299-312, 2014.

A. F. Brinkmann, E. van Zundert, and R. J. Saft. Herziening levenscyclusanalyse voor GFTafval: herberekening LCA bij het MER LAP (Revised life cycle analysis for VGF-waste: new LCA calculation of the EIA National Waste Management Policy Plan). Grontmij/ IVAM, De Bilt, Amsterdam, The Netherlands, 2004.

G. Chilosi, A. Esposito, F. Castellani, V. Stanzione, M. Aleandri, A. Tomassini, D. dell'Unto, A. Vannini, and R. Altieri. Characterization and use of olive mill waste compost as peat surrogate in substrate for cultivation of photinia potted plants: assessment of growth performance and in vitro suppressiveness. Waste and Biomass Valorization (doi:10.1007/s12649017-9855-7), 2017. 
J. Couwenberg. Greenhouse gas emissions from managed peat soils: is the ipcc reporting guidance realistic. Mires and Peat, 8(2):1-10, 2011.

O. Crespo, J. E. Bergez, and F. Garcia. Multiobjective optimization subject to uncertainty: Application to irrigation strategy management. Computers and Electronics in Agriculture, $74(1): 145-154,2010$.

Graeme J. Doole. Evaluation of an agricultural innovation in the presence of severe parametric uncertainty: An application of robust counterpart optimisation. Computers and Electronics in Agriculture, 84:16-25, 2012.

M. Ehrgott. Multicriteria Optimization. Springer, Berlin, Heidelberg, 2005.

M. Ehrgott, J. Ide, and A. Schöbel. Minmax robustness for multi-objective optimization problems. European Journal of Operational Research, 239:17-31, 2014.

G. Eichfelder, C. Krüger, and A. Schöbel. Decision uncertainty in multiobjective optimization. Journal of Global Optimization, 69(2):485-510, 2017.

J. Geldermann, R. Gabriel, and O. Rentz. Ecological assessment of the environmental impacts of the kerosene burning in jet turbines and its improvement assessment. Environmental science and pollution research international, 6(2):115-121, 1999.

J. Geldermann, L. M. Kolbe, A. Schöbel, and M. Schumann. Ressourceneffizienz in unternehmensnetzwerken - methoden zur betrieblichen und überbetrieblichen planung für die nutzung erneuerbarer rohstoffe. In Heinz Ahn, Marcel Clermont, and Rainer Souren, editors, Nachhaltiges Entscheiden. Beiträge zum multiperspektivischen Performancemanagement von Wertschöpfungsprozessen, pages 491-508. Springer Fachmedien Wiesbaden, Wiesbaden, 2016.

M. Goerigk and A. Schöbel. Algorithm engineering in robust optimization. In L. Kliemann and P. Sanders, editors, Algorithm Engineering: Selected Results and Surveys, volume 9220 of LNCS State of the Art, pages 245-279. 2016. URL http://arxiv.org/abs/1505.04901.

R. A. Gross and B. Kalra. Biodegradable polymers for the environment. Science, 297(5582): 803-807, 2002.

J.B. Guinée, M. Gorrée, R. Heijungs, G. Huppes, R. Kleijn, A. de Koning, L. van Oers, A. Wegener Sleeswijk, S. Suh, H.A. Udo de Haes, H. de Bruijn, R. van Duin, and M.A.J. Huijbregts. Handbook on life cycle assessment: Operational guide to the ISO standards. Eco-efficiency in industry and science, v. 7. Kluwer Academic Publishers, Dordrecht, The Netherlands, 2002.

K. Hayashi. Multicriteria analysis for agricultural resource management: A critical survey and future perspectives. European Journal of Operational Research, 122(2):486-500, 2000.

L. E. Hombach, C. Büsing, and G. Walther. Robust and sustainable supply chains under market uncertainties and different risk attitudes - a case study of the german biodiesel market. European Journal of Operational Research (in press), 2017.

T. A. Hottle, M. M. Bilec, and A. E. Landis. Sustainability assessments of bio-based polymers. Polymer Degradation and Stability, 98(9):1898-1907, 2013. 
J. Ide and A. Schöbel. Robustness for uncertain multi-objective optimization: A survey and analysis of different concepts. OR Spectrum, 38(1):235-271, 2016.

IPCC. Intergovernmental Panel on Climate Change. Climate Change 200\%: Synthesis Report. Contribution of Working Groups I, II and III to the Fourth Assessment Report of the Intergovernmental Panel on Climate Change. Edited by Pachauri, R. K. and Reisinger, A. Cambridge, UK, and New York, NY: Cambridge University Press., 2007.

P. Kouvelis and G. Yu. Robust Discrete Optimization and Its Applications. Nonconvex Optimization and its Applications. Kluwer Academic Publishers, Dordrecht, 1997.

M. Kranert, R. Gottschall, C. Bruns, G. Hafner, O. Schiere, C. Seibel, M. Dohmann, K. Fricke, M. Fricke, A. Karwatzki, E. Kraft, J. Lippross, K. Opphard, and T. Pretz. Grünabfälle besser kompostieren oder energetisch verwerten? - Vergleich unter den Aspekten der CO2Bilanz und der Torfsubstitution. EdDE-Dokumentation Nr. 11. Entsorgergemeinschaft der Deutschen Entsorgungswirtschaft e.V., Köln, Germany, 2007.

C. Krüger. Peat and pots: Analysis of robust solutions for a biobjective problem in agriculture. Technical Report 2018-5, Preprint-Reihe, Institut für Numerische und Angewandte Mathematik, Georg-August Universität Göttingen, 2018.

D. Kuroiwa and G. M. Lee. On robust multiobjective optimization. Vietnam Journal of Mathematics, 40(2\&3):305-317, 2012.

G. Lazzerini, S. Lucchetti, and F. P. Nicese. Analysis of greenhouse gas emissions from ornamental plant production: A nursery level approach. Urban Forestry $\&$ Urban Greening, 13(3):517-525, 2014.

G. Lazzerini, S. Lucchetti, and F. P. Nicese. Green house gases (ghg) emissions from the ornamental plant nursery industry: A life cycle assessment (lca) approach in a nursery district in central italy. Journal of Cleaner Production, 112:4022-4030, 2016.

T. J. Lowe and P. V. Preckel. Decision technologies for agribusiness problems: A brief review of selected literature and a call for research. Manufacturing $\&$ Service Operations Management, 6(3):201-208, 2004.

K. Miettinen. Nonlinear multiobjective optimization, volume 12. Springer, 2012.

J. R. Munhoz and R. Morabito. Optimization approaches to support decision making in the production planning of a citrus company: A brazilian case study. Computers and Electronics in Agriculture, 107:45-57, 2014.

S. Nambuthiri, A. Fulcher, A. K. Koeser, R. Geneve, and G. Niu. Moving toward sustainability with alternative containers for greenhouse and nursery crop production: A review and research update. HortTechnology, 25(1):8-16, 2015.

M. Papafotiou, G. Kargas, and I. Lytra. Olive-mill waste compost as a growth medium component for foliage potted plants. HortScience, 40(6):1746-1750, 2005.

A. Pardossi, L. Incrocci, G. Incrocci, F. Tognoni, and P. Marzialetti. What limits and how to improve water use efficiency in outdoor container cultivation of ornamental nursery stocks. Acta Horticulturae, (843):73-80, 2009. 
M. Raviv. Composts in growing media: feedstocks, composting methods and potential applications. Acta Horticulturae, (1018):513-523, 2014.

E. Riggi, G. Santagata, and M. Malinconico. Bio-based and biodegradable plastics for use in crop production. Recent Patents on Food, Nutrition \& Agriculturee, 3(1):49-63, 2011.

J. A. Robbins and M. R. Evans. Growing media for container production in a greenhouse or nursery. Cooperative Extension Service, University of Arkansas, US Department of Agriculture and county governments cooperating, 2001.

G. Scarascia-Mugnozza, C. Sica, and G. Russo. Plastic materials in european agriculture: actual use and perspectives. Journal of Agricultural Engineering, 42(3):15-28, 2011.

E. Schettini, G. Santagata, M. Malinconico, B. Immirzi, G. Scarascia-Mugnozza, and G. Vox. Recycled wastes of tomato and hemp fibres for biodegradable pots: Physico-chemical characterization and field performance. Resources, Conservation and Recycling, 70:9-19, 2013.

A. Schöbel. Generalized light robustness and the trade-off between robustness and nominal quality. MMOR, 80(2):161-191, 2014.

J.-S. Shih and H. C. Frey. Coal blending optimization under uncertainty. European Journal of Operational Research, 83(3):452-465, 1995.

J. M. Waddington, J. Plach, J. P. Cagampan, M. Lucchese, and M. Strack. Reducing the carbon footprint of canadian peat extraction and restoration. AMBIO: A Journal of the Human Environment, 38(4):194-200, 2009.

M. M. Wiecek and G. M. Dranichak. Robust Multiobjective Optimization for Decision Making Under Uncertainty and Conflict, chapter 4, pages 84-114. INFORMS, 2016.

M. R. Yates and C. Y. Barlow. Life cycle assessments of biodegradable, commercial biopolymers - a critical review. Resources, Conservation and Recycling, 78:54-66, 2013. 
D. Peat and pots: Analysis of Robust Solutions for a Biobjective Problem in Agriculture

C. Krüger 
D. Peat and pots: Analysis of Robust Solutions for a Biobjective Problem in Agriculture 


\title{
Peat and pots: Analysis of robust solutions for a biobjective problem in agriculture
}

\author{
Corinna Krüger \\ Institute for Numerical and Applied Mathematics, University of Goettingen, \\ Lotzestr. 16-18, 37083 Göttingen, Germany
}

\begin{abstract}
Robust multiobjective optimization is used to solve an uncertain biobjective mixing problem arising in the commercial cultivation of potted plants. The so-called peat-andpots problem is to search a mixture of two types of growth substrate and a planter pot such that two objectives, one economic and one ecological, are minimized. The economic objective function is the sum of a linear function and a piecewise constant function while the ecological objective function is linear. In practice, the problem is affected by two types of uncertainty, parameter and decision uncertainty. Parameter uncertainty means that the problem data varies within a fixed set, e.g., the emissions of the growth substrate are known to fall in a certain interval. Decision uncertainty means that solutions can not be implemented exactly as targeted, e.g., the mixing of growth substrate is performed roughly which leads to different mixtures in different pots. The peat-and-pots problem is considered first as a deterministic problem and the deterministic efficient solutions are determined. Second, the two types of uncertainty are taken into account and the problem is addressed by means of robust multiobjective optimization. Analytical descriptions of the set of solutions to the deterministic and to the uncertain problem are given. The deterministic and robust solution approaches are then extended to further cases of biobjective problems with one objective function being the sum of a linear and a piecewise constant function and the second objective function being linear.
\end{abstract}

\section{Introduction}

Robust multiobjective optimization is a research area that has recently been developed to address uncertain multiobjective optimization problems, that is, problems with two or more conflicting objective functions that are affected by uncertainty. Two types of uncertainty are generally distinguished. When the problem parameters are not known exactly we speak of parameter uncertainty. Parameter uncertainty can result from by fluctuations of costs or imprecisions of experimental data. The second type of uncertainty, decision uncertainty, occurs whenever solutions are not put into practice exactly as planned but within a range of the intended value.

Robustness is particularly useful as an approach to address uncertainty if the uncertainty set (i.e., the set of scenarios) is known and if there is no information on the distribution of the scenarios. Various concepts of robustness for multiobjective optimization have been proposed in the literature, see $[17,27]$ for recent surveys, and many multiobjective robustness concepts are based on single-objective robustness concepts. One of the most studied robustness concepts is minmax robustness. Robust optimization searches solutions that are feasible 
for all scenarios in the uncertainty set and robust optimal solutions are those that perform best with respect to worst case scenarios. In multiobjective optimization, there are several ideas of a worst-case scenario and various multiobjective minmax robustness concepts have been proposed and researched in the literature, e.g., see [19, 9] for minmax robustness for parameter uncertainty, and see [10] for minmax robustness for decision uncertainty.

Robust multiobjective optimization has many applications and it is of particular interest for agricultural decision problems. A review of approaches to uncertain problems in agriculture is given in [6] and robust optimization has been applied to various optimization problems in agriculture, see, e.g., $[4,8]$. While these studies have taken into account a single objective function, multiobjective optimization is more appropriate for many agricultural decision problems and a survey on multiobjective optimization in agriculture is given in [15]. Two frequently combined types of objective functions are economic objective functions such as costs or profit, and environmental objective functions such as the global warming potential or greenhouse gas emissions, see e.g., [14].

The uncerain multiobjective problem from agriculture considered in this paper is a biobjective mixing problem that is called the peat-and-pots problem and it is given as follows: A plant nursery aims to simultaneously minimize the costs and the environmental impacts of potted plant cultivation. To this end, the nursery selects the growth substrate and the planter pots required for the cultivation of potted plants. The growth substrate can be chosen as any mixture of peat and compost, and traditionally, $100 \%$ peat is used. Planter pots can be chosen as plastic pots, which are used traditionally, or biodegradable pots.

In the economic objective, the additional costs, the costs of the input materials and the selling price of the plant are considered, and the impact of the plant's quality on its selling price is taken into account. The corresponding quality function that maps the choice of growth substrate and pot to the quality level of the plant is piecewise constant and upper semicontinuous. The peat-and-pots problem is affected by two types of uncertainty, parameter uncertainty and decision uncertainty. Parameter uncertainty is present, since the parameters for the costs and emissions are not known in advance but they are known to fall in given intervals and decision uncertainty affects the mixing of the growth substrate since the different ingredients are mixed roughly.

In this work, robust multiobjective optimization is used to determine solutions to the uncertain bi-objective peat-and-pots problem, which are subsequently called decision robust efficient solutions. This multiobjective robustness concept is introduced and studied in [10] and decision robust efficient solutions are defined as solutions to the robust counterpart that is a deterministic problem associated with this robustness concept. The major difficulty of determining decision robust efficient solutions therefore lies in solving the robust counterpart that is a set-valued optimization problem here. In the literature, applications of minmax robust multiobjective optimization to problems with one monetary and one environmental objective function are so far restricted to robustness concepts where the robust counterpart is considered a deterministic multiobjective problem, see, e.g., [16, 21]. To the best of our knowledge, this is the first application of a multiobjective robustness concept with a setvalued robust counterpart to such a problem. The presented results may serve as a guidance for managers of the horticulture sector before the beginning of horticultural seasonal activities, and the findings can be extended to further applications of uncertain mixing problems. Also note that this research is motivated by and applied to a case study in [18], where it is found that the robust solutions are preferable over the deterministic ones.

The paper is structured as follows: In Section 2, the peat-and-pots problem is motivated 
from a practical viewpoint and the problem is introduced in its deterministic form and its two objective functions are described in detail. In Section 3, an analytical description of the deterministic efficient solutions to the peat-and-pots problem is given. In Section 4, parameter uncertainty and decision uncertainty in the peat-and-pots problem are taken into account and an analytical description of the decision robust efficient solutions to the uncertain peat-andpots problem is presented. In Section 5, the presented results are extended to related decision problems that involve one objective function that is the sum of a piecewise constant and a linear function and one linear objective function. In Section 6, a summary and an outlook for further research is given.

\section{Problem description}

The following decision problem is motivated by a case study on potted plant production for the species Photinia $x$ Fraseri that is explained in detail in [18]. Note that the description of the problem, its two objective functions and the uncertainty therein corresponds in large parts to the description in the former publication, since the present work is meant to provide solution approaches for the case study in the former publication.

A major environmental problem in European horticulture is the greenhouse gas (GHG) emissions caused by potted plant cultivation using peat and plastics [20]. Peat is a very flexible material that can be adapted for most plants, see [24]. However, peatlands (or bogs) are important carbon sinks, and peat mining and extraction result in significant emissions of the GHGs carbon dioxide, methane, and nitrous oxide [26]. Peatland protection is also considered vital for maintaining biodiversity. To reduce the environmental impacts of potted plant production, alternative growth media made of renewable resources or by-products can be used to replace peat such as compost, bark, wood, rice hulls or animal manures.

In horticulture settings, peat could be best replaced by compost. Beyond a certain replacement percentage, however, the agronomic quality of the potted plant becomes unacceptable [22]. Agricultural supply chains generate a great variety and quantity of waste and byproducts that could be used as compost, including the by-products from the olive oil industry [23]. The latter is also considered in the horticultural case study in [18] that motivates this investigation

In horticulture, plastic pots are widely used because of their low cost, durability, and versatility. Since they cannot be recycled easily due to soil and vegetable matter contamination, agrochemical residues, and additives, they are generally landfilled after one usage [25]. Here, biodegradable pots, which can be embedded in the soil with the plant or disposed of in composting facilities, represent a viable alternative to plastic pots.

From a responsible management perspective, it makes sent to consider both peat substitution and the replacement of plastic pots with biodegradable ones. The resulting reduction in GHG emissions can be determined by a Life Cycle Assessment (LCA), which looks at environmental impacts from all stages of a product's life, including raw material extraction, materials processing, manufacturing, distribution, use, repair, maintenance, and disposal or recycling, see, e.g., $[12,7]$

In addition to the minimization of GHG-emissions, the minimization of the additional costs caused by the substitution of peat and plastic pots has to be taken into account. The additional costs are considered here as the difference between the costs of the decision relevant input materials and the selling price of the potted plant. This leads to a bi-objective 
optimization problem, which is subsequently called the peat-and-pots problem.

\section{Modelling the Peat-and-Pots Problem}

The aim of the peat-and-pots problem is to chose a share of compost to substitute peat and to chose either plastic or biodegradable pots for plant cultivation such that the additional costs $(a d C)$ and the global warming potential $(G W P)$ are minimized. The peat-and-pots problem is hence modelled as a bi-objective problem. We let one potted plant be the functional unit of the problem and assume it is representative of all plants of the same species cultivated in a given nursery. For each potted plant, we must decide on

- the share of compost $\gamma \in[0,1]$ to use for replacing peat,

- which of the two possible types of pots to use. This decision is modeled by introducing the pot variable $\beta \in\{0,1\}$, where $\beta=\left\{\begin{array}{ll}1 & \text { if bio-pot chosen } \\ 0 & \text { if plastic pot chosen }\end{array}\right.$.

The peat-and-pots problem for each species is a bi-objective minimization problem with two constraints that is given as follows:

$$
\begin{array}{ll}
\min & \left(\begin{array}{c}
a d C(\gamma, \beta) \\
G W P(\gamma, \beta)
\end{array}\right) \\
\text { s.t. } & Q(\gamma, \beta) \geq \\
\gamma & \geq v^{c o m p} \\
\gamma & \in[0,1] \\
\beta & \in\{0,1\} .
\end{array}
$$

In the first constraint, plants that fail to meet the aesthetic requirements for sale are excluded. The function $Q$ denotes the quality of a potted plant and a minimum quality of $\alpha \in \mathbb{N}$ is mandatory for the market. In the second constraint, we require that the quantity of compost $\gamma$ does not exceed the available quantity of compost per pot $v^{\text {comp }}$.

The feasible set of $\mathcal{P}$ is given as

$$
\mathfrak{F}:=\left\{(\gamma, \beta) \in[0,1] \times\{0,1\} \mid 0 \leq \gamma \leq \min \left\{1, v^{\operatorname{comp}}\right\}, Q(\gamma, \beta) \geq \alpha\right\} .
$$

The first objective function represents the additional costs $(a d C)$, which are to be minimized. The second objective function represents the global warming potential $(G W P)$, which is also to be minimized. We first describe both objectives in detail before we solve the deterministic biobjective peat-and-pots problem.

\subsection{The economic objective: additional costs}

The first objective, the additional costs, has two parts: the purchase costs of the input materials (growth media and pot) and the selling price of the potted plant. All other costs can be neglected, since they are independent of the decision problem being considered here.

As a part of the additional costs function, we hence obtain the function costs: $[0,1] \times$ $\{0,1\} \rightarrow \mathbb{R}$

$$
\begin{aligned}
\operatorname{costs}(\gamma, \beta) & =c^{\text {peat }} \cdot(1-\gamma)+c^{\text {comp }} \cdot \gamma+c^{\text {plas }} \cdot(1-\beta)+c^{\text {bio }} \cdot \beta \\
& =c^{\text {peat }}+c^{\text {plas }}+\gamma \cdot\left(c^{\text {comp }}-c^{\text {peat }}\right)+\beta \cdot\left(c^{\text {bio }}-c^{\text {plas }}\right),
\end{aligned}
$$


where the parameters $c^{\text {peat }}, c^{c o m p}, c^{\text {plas }}, c^{\text {bio }}$ denote the costs of one functional unit of peat, one functional unit of compost, a plastic pot, and a biodegradable pot, respectively. Because this study aims to model and solve the peat-and-pots problem such that it meets the specific requirements of the case study in [18], we subsequently describe these requirements in specific assumptions. This case study yields the observation $c^{\text {peat }}>c^{\text {comp }}$, which is reflected by the following assumption.

Assumption 1. The cost of compost is strictly smaller than the cost of peat, i.e.,

$$
c^{\text {peat }}>c^{c o m p} .
$$

As a consequence, we get that the cost function costs is strictly decreasing in $\gamma$ for every $\beta \in\{0,1\}$.

The selling price of a potted plant depends on its quality, and each potted plant is assigned one out of a fixed number of specific quality levels. The lower the quality level, the lower is the selling price of the potted plant. Replacing peat with compost changes the agronomic quality of the plant, see [22]. We assume that the quality function $Q$ assigns one out of $\nu$ quality levels to each decision, i.e.,

$$
Q:[0,1] \times\{0,1\} \rightarrow\{1, \ldots, \nu\},
$$

where $\nu$ is the maximum possible quality level. The quality function $Q$ is piecewise constant for each $\beta \in\{0,1\}$, where the bounds of the constant parts of the quality function are

$$
0=t_{0}^{\beta}<t_{1}^{\beta}<t_{2}^{\beta}<\cdots<t_{\omega}^{\beta}=1 .
$$

For every $1 \leq i \leq \omega$, where $\omega$ is the number of constant intervals, we have $q_{i} \in\{0, \ldots, \nu\}$ such that $Q(\gamma, \beta)=q_{i}$ for all $\gamma \in\left(t_{i-1}^{\beta}, t_{i}^{\beta}\right)$. The quality function $Q$ is then given as

$$
Q(\gamma, \beta)= \begin{cases}q_{i}, & \text { if } \gamma \in\left(t_{i-1}^{\beta}, t_{i}^{\beta}\right), \text { for } i=1, \ldots, \omega \\ \max \left\{q_{i}, q_{i+1}\right\} & \text { if } \gamma=t_{i}^{\beta} \text { for } i=1, \ldots, \omega-1 .\end{cases}
$$

The solutions $t_{0}^{\beta}=0$ and $t_{\omega}^{\beta}=1$ are boundary points for only one interval and their quality is defined as $Q\left(t_{0}^{\beta}, \beta\right)=q_{1}$ and $Q\left(t_{\omega}^{\beta}, \beta\right)=q_{\omega}$, repectively. Hence, by definition, the quality function $Q$ is not continuous but upper semicontinuous for every given pot variable $\beta \in\{0,1\}$. Figure 1 illustrates the dependence of the quality on the share of compost for the species Photinia in the case study given in [18], where $\nu=5$ quality levels are distinguished.

Dashed and straight lines in Figure 1 demonstrate how the quality function $Q$ depends on the choice of planter pots. It is assumed that plants raised in biodegradable pots (straight lines) are of better or equal quality than plants raised in plastic pots (dashed lines).

Each quality level can be associated to a selling price. We assume that a given number of quality levels can be attained, but that only levels high enough are suitable for selling. The minimum quality level for sale is denoted as $\alpha \in\{0, \ldots, \nu\}$. We furthermore assume that plants that are raised on the traditional growth media ( $100 \%$ peat, i.e., $\gamma=0$ ) can be sold in general, and hence, we have $\alpha \leq Q(0, \beta)$ for every $\beta$. The relationship between the quality and the price (in $[€]$ ) of a potted plant depends on the species and is defined by the function price,

$$
\text { price: }\{1, \ldots, \nu\} \rightarrow \mathbb{R}, \quad q \mapsto \operatorname{price}(q) .
$$




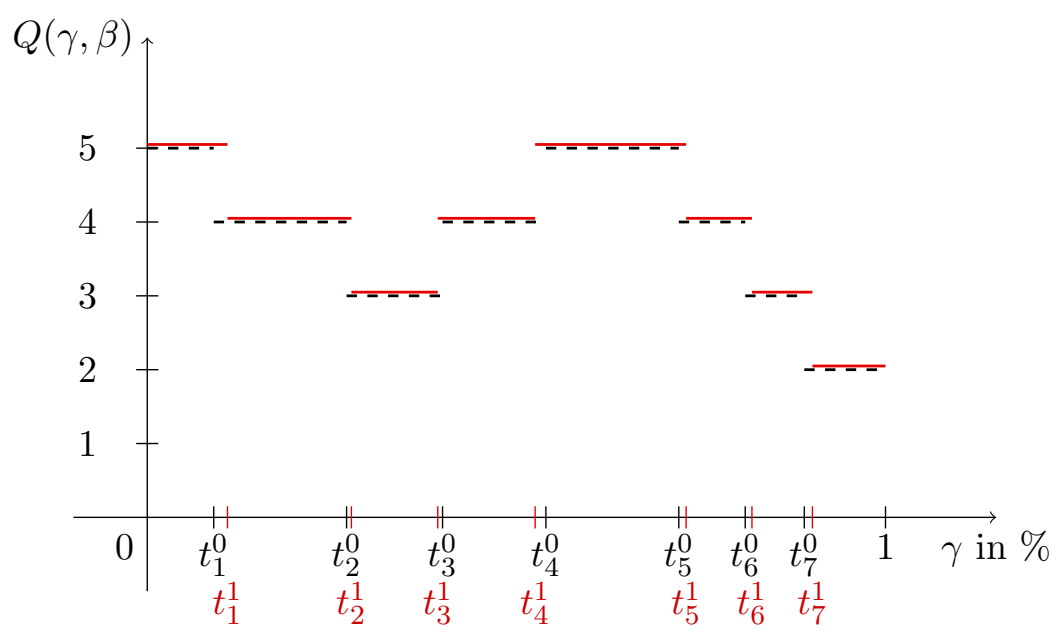

Figure 1: Quality function for the species Photinia $x$ Fraseri. Dashed black lines indicate a plastic pot $(\beta=0)$ and straight lines indicate a biodegradable pot $(\beta=1)$.

The first objective $a d C$ is obtained by combining the selling price and the costs

$$
\operatorname{adC}(\gamma, \beta):=\operatorname{costs}(\gamma, \beta)-\operatorname{price}(Q(\gamma, \beta)) .
$$

For general quality functions, we can without loss of generality assume that the price of a salable product increases with its quality, since the quality function can always be chosen accordingly. We hence have for every $\left(\gamma_{i}, \beta_{i}\right),\left(\gamma_{j}, \beta_{j}\right) \in \mathfrak{F}$ such that $Q\left(\gamma_{i}, \beta_{i}\right)<Q\left(\gamma_{j}, \beta_{j}\right)$,

$$
\operatorname{price}\left(Q\left(\gamma_{i}, \beta_{i}\right)\right)<\operatorname{price}\left(Q\left(\gamma_{j}, \beta_{j}\right)\right) \text {. }
$$

This implies that the first objective $a d C$ is strictly decreasing on each of the intervals $\left(t_{i-1}^{\beta}, t_{i}^{\beta}\right]$ for $i=1, \ldots, \omega$, as shown in Lemma 1 .

Lemma 1. (i) Let $\left(\gamma_{i}, \beta\right),\left(\gamma_{j}, \beta\right) \in \mathfrak{F}$ be such that $\gamma_{i}<\gamma_{j}$ and $Q\left(\gamma_{i}, \beta\right) \leq Q\left(\gamma_{j}, \beta\right)$, then we have

$$
\operatorname{adC}\left(\gamma_{i}, \beta\right)>\operatorname{adC}\left(\gamma_{j}, \beta\right) .
$$

(ii) For every $\beta \in\{0,1\}$, the objective function, adC is monotonically strictly decreasing on each of the intervals $\left(t_{i-1}^{\beta}, t_{i}^{\beta}\right]$ for $i=1, \ldots, \omega$.

Proof. We first prove (i). Using (2) and (3), we obtain

$$
\begin{aligned}
& a d C\left(\gamma_{i}, \beta\right)-a d C\left(\gamma_{j}, \beta\right)=\underbrace{\left(\gamma_{j}-\gamma_{i}\right)}_{>0} \cdot \underbrace{\left(c^{\text {peat }}-c^{\text {comp }}\right)}_{>0}+\underbrace{(\beta-\beta)}_{=0} \cdot\left(c^{\text {plas }}-c^{\text {bio }}\right) \\
& +\underbrace{\operatorname{price}\left(Q\left(\gamma_{j}, \beta\right)\right)-\operatorname{price}\left(Q\left(\gamma_{i}, \beta\right)\right)}_{\geq 0} \\
& >0,
\end{aligned}
$$

where $0<c^{\text {peat }}-c^{\text {comp }}$ by Assumption 1. Claim (ii) follows from (i) because the quality function $Q$ is constant on $\left(t_{i-1}^{\beta}, t_{i}^{\beta}\right)$ and upper semicontinuous on $\left(t_{i-1}^{\beta}, t_{i}^{\beta}\right]$ by definition. 
Based on the data for the species Photinia in [18], we observe that the differences between the costs of the input materials are small when compared to the differences of the selling prices between different quality levels. Hence, for the remainder of this study, we pose the following assumption.

Assumption 2. For all $\left(\gamma_{i}, \beta_{i}\right),\left(\gamma_{j}, \beta_{j}\right) \in \mathfrak{F}$ such that $Q\left(\gamma_{i}, \beta_{i}\right)<Q\left(\gamma_{j}, \beta_{j}\right)$, it holds

$$
\left|\operatorname{costs}\left(\gamma_{j}, \beta_{j}\right)-\operatorname{costs}\left(\gamma_{i}, \beta_{i}\right)\right|<\operatorname{price}\left(Q\left(\gamma_{j}, \beta_{j}\right)\right)-\operatorname{price}\left(Q\left(\gamma_{i}, \beta_{i}\right)\right) .
$$

This directly implies that a loss of quality causes an increase of the additional costs, as stated in the next lemma.

Lemma 2. Let $\left(\gamma_{i}, \beta_{i}\right),\left(\gamma_{j}, \beta_{j}\right) \in \mathfrak{F}$ be such that $Q\left(\gamma_{i}, \beta_{i}\right)<Q\left(\gamma_{j}, \beta_{j}\right)$, then we have

$$
\operatorname{adC}\left(\gamma_{i}, \beta_{i}\right)>\operatorname{adC}\left(\gamma_{j}, \beta_{j}\right) .
$$

\subsection{The environmental objective: global warming potential}

The second objective function GWP considers the decision-relevant emissions of a potted plant. For each feasible share of compost $\gamma$, the GWP is the linear combination of the corresponding values for $100 \%$ peat and $100 \%$ compost:

$$
\begin{aligned}
G W P(\gamma, \beta) & =e m^{\text {peat }} \cdot(1-\gamma)+e m^{c o m p} \cdot \gamma+e m^{\text {plas }} \cdot(1-\beta)+e m^{\text {bio }} \cdot \beta \\
& =e m^{\text {peat }}+e m^{\text {plas }}+\gamma \cdot\left(e m^{\text {comp }}-e m^{\text {peat }}\right)+\beta \cdot\left(e m^{\text {bio }}-e m^{\text {plas }}\right),
\end{aligned}
$$

where $e m^{\text {peat }}, e m^{c o m p}, e m^{\text {plas }}, \mathrm{em}^{\text {bio }}$ denote the GWP of one functional unit of peat or compost and the GWP of a plastic or biodegradable pot. By means of the data provided by the case study in [18],we observe that the GWP of compost and biodegradable pots is smaller than the GWP of peat and plastic pots (with respect to the functional unit that is one potted plant) and we pose this as the following assumption.

Assumption 3. The GWP of compost is strictly smaller than the GWP of peat, and the GWP of a biodegradable pot is strictly smaller than the GWP of a plastic pot, i.e.,

$$
e m^{\text {comp }}<e m^{\text {peat }} \text { and } e m^{\text {bio }}<e m^{\text {plas }} .
$$

We directly conclude that $G W P$ is strongly decreasing in $(\gamma, \beta)$ as shown in Lemma 3.

Lemma 3. Let $\left(\gamma_{i}, \beta_{i}\right),\left(\gamma_{j}, \beta_{j}\right) \in \mathfrak{F}$ be such that $\gamma_{i} \leq \gamma_{j}, \beta_{i} \leq \beta_{j}$ and $\left(\gamma_{i}, \beta_{i}\right) \neq\left(\gamma_{j}, \beta_{j}\right)$. Then we have

$$
G W P\left(\gamma_{j}, \beta_{j}\right)<G W P\left(\gamma_{i}, \beta_{i}\right) .
$$

Proof. By Assumption 3, we have

$$
\begin{aligned}
G W P(\gamma, \beta) & =e m^{\text {peat }} \cdot(1-\gamma)+e m^{\text {comp }} \cdot \gamma+e m^{\text {plas }} \cdot(1-\beta)+e m^{\text {bio }} \cdot \beta \\
& =\underbrace{e m^{\text {peat }}+e m^{\text {plas }}}_{\text {const. }}+\gamma \cdot \underbrace{\left(e m^{\text {comp }}-e m^{\text {peat }}\right)}_{<0}+\beta \cdot \underbrace{\left(e m^{\text {bio }}-e m^{\text {plas }}\right)}_{<0} .
\end{aligned}
$$

The claim follows immediately. 


\section{Deterministic efficient solutions to the peat-and-pots prob- lem}

We use the well-known classical optimality concept (Pareto-)efficiency to define optimal solutions to the bi-objective problem $\mathcal{P}$. For all $y, z \in \mathbb{R}^{2}$ the Pareto order relations are given by $y \leqq z \Leftrightarrow y_{1} \leq z_{1}$ and $y_{2} \leq z_{2} ; y \leq z \Leftrightarrow y \leqq z$ and $y \neq z$; and $y<z \Leftrightarrow y_{1}<z_{1}$ and $y_{2}<z_{2}$.

Definition 4. A solution $x^{*} \in \mathfrak{F}$ is called [weakly/./strictly] (Pareto-)efficient for $\mathcal{P}$ if there exists no solution $x \in \mathfrak{F} \backslash\left\{x^{*}\right\}$ such that

$$
(\operatorname{adC}(x), G W P(x))^{T}[</ \leq / \leqq]\left(\operatorname{adC}\left(x^{*}\right), G W P\left(x^{*}\right)\right)^{T} .
$$

The peat-and-pots problem is a mixed integer problem and the basic idea for solving it is to solve it separately for $\beta=0$ and for $\beta=1$. The efficient solutions can then be determined by a pairwise comparison of the efficient solutions for the two values of the pot variable $\beta \in\{0,1\}$. As the results of this section show, the number of efficient solutions for each $\beta \in\{0,1\}$ is small, and hence, the comparison of the efficient solutions for different pot variables can be performed with low computational effort. We next determine analytical descriptions of the [weakly/ / strictly] efficient solutions to the deterministic peat-and-pots problem for fixed $\beta$.

As a first result we show that the weakly efficient solutions coincide with the strictly efficient solutions of $\mathcal{P}$.

Proposition 5. Let $\left(\gamma_{i}, \beta\right),\left(\gamma_{j}, \beta\right) \in \mathfrak{F}$ with $\gamma_{i} \neq \gamma_{j}$ be two different solutions. Then

$$
\begin{aligned}
& \left(\begin{array}{c}
a d C\left(\gamma_{i}, \beta\right) \\
G W P\left(\gamma_{i}, \beta\right)
\end{array}\right)<\left(\begin{array}{c}
a d C\left(\gamma_{j}, \beta\right) \\
G W P\left(\gamma_{j}, \beta\right)
\end{array}\right) \\
\Leftrightarrow \quad & \left(\begin{array}{c}
a d C\left(\gamma_{i}, \beta\right) \\
G W P\left(\gamma_{i}, \beta\right)
\end{array}\right) \leqq\left(\begin{array}{c}
a d C\left(\gamma_{j}, \beta\right) \\
G W P\left(\gamma_{j}, \beta\right)
\end{array}\right) .
\end{aligned}
$$

Proof. It is sufficient to show that the second inequality implies the first. Let the second inequality be valid. Applying Lemma 3 and the assumption $\gamma_{i} \neq \gamma_{j}$, we obtain $\gamma_{j}<\gamma_{i}$. We also have $Q\left(\gamma_{j}, \beta\right) \leq Q\left(\gamma_{i}, \beta\right)$, because otherwise $a d C\left(\gamma_{j}, \beta\right)<a d C\left(\gamma_{i}, \beta\right)$ by Lemma 2 . The strict inequality then follows from Lemma 1.

The set of feasible solutions with respect to the fixed pot variable $\beta$ is denoted as

$$
\mathfrak{F}(\beta):=\{\gamma \mid(\gamma, \beta) \in \mathfrak{F}\} .
$$

The set $\mathfrak{F}(\beta)$ is a compact set because the quality function $Q$ is upper semicontinuous. Hence, the maximum element of $\mathfrak{F}(\beta)$ exists and we denote it as $t_{\text {max }}^{\beta}:=\max _{\gamma \in \mathfrak{F}(\beta)} \gamma$. The quality level of $t_{\text {max }}^{\beta}$ is denoted as $\nu_{\min }:=Q\left(t_{\max }^{\beta}, \beta\right)$.

In the next theorem, an analytical description of the set of efficient solutions to the deterministic problem for the fixed pot variable $\beta$ is given.

Theorem 6. For each fixed pot variable $\beta \in\{0,1\}$, the set

$$
\mathcal{D}(\beta):=\bigcup_{j=\nu_{\min }}^{\nu}\{\max \{\gamma \in \mathfrak{F}(\beta) \mid Q(\gamma, \beta) \geq j\}\},
$$

is the set of efficient solutions. 
Proof. We first show that the set $\mathcal{D}(\beta)$ is well-defined. Because of the upper semicontinuity of the quality function $Q$, the set $\{\gamma \in \mathfrak{F}(\beta) \mid Q(\gamma, \beta) \geq j\}$ is closed for every $j=\nu_{\min }, \ldots, \nu$, and hence, it is compact as a subset of a compact interval. Therefore, the maximum of the set exists whenever it is not empty and $\mathcal{D}(\beta)$ is well-defined.

We next show that the set of efficient solutions is a subset of $\mathcal{D}(\beta)$ by showing that every solution in $\mathfrak{F}(\beta) \backslash \mathcal{D}(\beta)$ is not efficient. For every $\gamma^{\prime} \in \mathfrak{F}(\beta) \backslash \mathcal{D}(\beta)$ with $j^{\prime}:=Q\left(\gamma^{\prime}, \beta\right)$, we define

$$
\gamma^{\dagger}:=\left\{\begin{array}{ll}
\max \left\{t \in \mathfrak{F}(\beta) \mid Q(t, \beta) \geq j^{\prime}\right\} & \text { if } \nu_{\min } \leq j^{\prime} \\
t_{\max }^{\beta} & \text { if } \nu_{\min }>j^{\prime}
\end{array} .\right.
$$

We have $\gamma^{\dagger} \in \mathcal{D}(\beta)$ by definition and $\gamma^{\dagger}$ satisfies $\gamma^{\prime} \leq \gamma^{\dagger}$ and $Q\left(\gamma^{\dagger}, \beta\right) \geq j^{\prime}$. Because $\gamma^{\prime} \leq \gamma^{\dagger}$ and $\gamma^{\prime} \notin \mathcal{D}(\beta)$, we obtain $\gamma^{\prime}<\gamma^{\dagger}$, and, according to Lemma $1, \gamma^{\prime}$ is dominated by $\gamma^{\dagger}$, and hence, $\gamma^{\prime}$ is not efficient.

We finally show that every element of $\mathcal{D}(\beta)$ is efficient. For every $\bar{\gamma} \in \mathcal{D}(\beta)$, there exists $j \in\left\{\nu_{\min }, \ldots, \nu\right\}$ such that

$$
\bar{\gamma}=\max \{\gamma \in \mathfrak{F}(\beta) \mid Q(\gamma, \beta) \geq j\}
$$

by the definition of $\mathcal{D}(\beta)$. For a contradiction, we assume that there exists $\gamma^{\circ} \in \mathfrak{F}(\beta) \backslash \bar{\gamma}$ that dominates $\bar{\gamma}$. By Lemma 3, we directly obtain $\bar{\gamma}<\gamma^{\circ}$ and hence, $Q\left(\gamma^{\circ}, \beta\right)<j=Q(\bar{\gamma}, \beta)$ by definition of $\bar{\gamma}$. According to Lemma 2 we then have $a d C(\bar{\gamma}, \beta)<a d C\left(\gamma^{\circ}, \beta\right)$ in contradiction to the assumption that $\gamma^{\circ}$ dominates $\bar{\gamma}$.

By Theorem 6 , there exists at most one efficient solution for each quality $i=\nu_{\min }, \ldots, \nu$. Because the set $\mathcal{D}(\beta)$ is finite and nonempty for every $\beta \in\{0,1\}$, we directly obtain the existence of efficient solutions to the deterministic peat-and-pots problem.

Corollary 7. There always exists a (deterministic) efficient solution to $(\mathcal{P})$.

Theorem 6 confirms the intuitive approach of taking the maximum share of compost in a plant nursery: for every quality level, we consider all intervals of the quality function that have a higher or equal quality level and we choose the maximum share of compost therein. Therefore, the set of deterministic efficient solutions is easily computable by comparing the boundary points of the quality function's constant intervals.

\section{Uncertainty in the peat-and-pots problem}

Most decision problems involving natural resources are affected by uncertainty. In practice, the peat-and-pots problem is affected by parameter uncertainty and decision uncertainty. Parameter uncertainty means that a part of the problem data is uncertain. In the case study that motivates this work, the costs and emissions are known to fall in intervals, rather than being known as exact numbers. Decision uncertainty concerns the variations in the implementation of the calculated solution. In the case study, the targeted share of compost can not be implemented exactly, because the mixing of peat and compost is performed only roughly.

In the literature, parameter uncertainty is also known as data uncertainty and decision uncertainty is often referred to as the implementation error, see, e.g., $[2,1,3]$. 


\subsection{Parameter uncertainty in the peat-and-pots problem}

Various robustness concepts for parameter uncertainty in bi-objective or multiobjective problems have been introduced in the literature. In each case, it is assumed that there is a so-called uncertainty set containing all possible values of the data. Each element of the uncertainty set is called a scenario. In the case study, each parameter $c^{\text {peat }}, c^{\text {comp }}, c^{\text {plas }}, c^{\text {bio }}$ and $e m^{\text {peat }}, e m^{\text {comp }}, e m^{\text {plas }}, e m^{\text {bio }}$ falls within an interval, for example $c^{\text {peat }} \in\left[\underline{c}^{\text {peat }}, \bar{c}^{\text {peat }}\right]$, and the intervals are independent of each other, see [18]. We hence consider the uncertainty set

$$
\mathcal{U}=\left\{(c, e m) \mid c^{\text {peat }} \in\left[\underline{c}^{\text {peat }}, \bar{c}^{\text {peat }}\right], \cdots, e m^{b i o} \in\left[\underline{\mathrm{em}}^{b i o}, \overline{\mathrm{em}}^{\text {bio }}\right]\right\} .
$$

The first objective function $a d C$ is affected only by the cost parameters $(c)$ while the second objective function $(G W P)$ is affected only by the emission parameters. This is a special situation in uncertain multiobjective optimization that is called objective-wise uncertainty. In this case, two frequently studied concepts of minmax robustness for multiobjective problems coincide. These two are point-based minmax robust efficiency, see, e.g., [19, 11, 13], and setbased minmax robust efficiency, see, e.g., [9, 5]. The peat-and-pots problem has the property that there exists the worst-case parameter scenario $(\bar{c}, \overline{e m}) \in \mathcal{U}$, such that for every feasible solution $(\gamma, \beta)$ and for all parameter values $(c, e m) \in \mathcal{U}$ it holds

$$
a d C(\gamma, \beta, c) \leq a d C(\gamma, \beta, \bar{c}) \text { and } G W P(\gamma, \beta, e m) \leq G W P(\gamma, \beta, \overline{e m}) .
$$

As a consequence of this property and due to the nature of robust optimization to take into account the worst-case scenarios, it can be shown that a solution $\left(\gamma^{*}, \beta^{*}\right)$ is point-based or set-based minmax robust efficient, if and only if it is an efficient solution to the biobjective peat-and-pots problem for the scenario $(\bar{c}, \overline{e m}) \in \mathcal{U}$. The robust efficient solutions can therefore be determined by solving the peat-and-pots problem for the worst-case scenario, which is a deterministic bi-objective problem. The peat-and-pots problem can then be treated as unaffected by parameter uncertainty, since the uncertainty is included in the problem's objective functions.

\subsection{Decision uncertainty in the peat-and-pots problem}

Since, for our problem, it is reasonable to assume that a worker uses exactly the chosen type of pot, the only decision uncertainty is the share of compost in the substrate. In horticulture, growth substrate is prepared for many potted plants at one time, and the mixing is done roughly in big heaps using skid-steer loaders. The maximum and minimum deviation in compost share $\gamma$ that can be expected in a single pot can be estimated by the plant nursery based on experience (e.g., $10 \%$ lower or $10 \%$ higher than intended).

For our problem, we assume that both a minimum deviation $z^{\text {min }} \leq 0$ and a maximum deviation $0 \leq z^{\max }$ are given. The actual values of $\gamma$ are expected to fall within $\left[\gamma+z^{\min }, \gamma+\right.$ $\left.z^{\max }\right]$. However, this is not correct for values $\gamma<-z^{\min }$ or $\gamma>1-z^{\max }$, since a share below $0 \%$ or above $100 \%$ is not permitted. The uncertainty when implementing a decision hence depends on the values of $\gamma$. It is defined as

$$
\begin{aligned}
& z^{\min }(\gamma):= \begin{cases}\max \left\{z^{\min },-\gamma\right\} & \text { for } \gamma \in(0,1) \\
0 & \text { for } \gamma \in\{0,1\}\end{cases} \\
& z^{\max }(\gamma):= \begin{cases}\min \left\{z^{\max }, 1-\gamma\right\} & \text { for } \gamma \in(0,1) \\
0 & \text { for } \gamma \in\{0,1\}\end{cases}
\end{aligned}
$$


to yield the deviation set $Z(\gamma)$ of $\gamma \in[0,1]$ as

$$
Z(\gamma):=\left[z^{\min }(\gamma), z^{\max }(\gamma)\right]
$$

The realized values for every $\gamma \in(0,1)$ are finally obtained in

$$
\{\gamma\}+Z(\gamma)=\left[\max \left\{\gamma+z^{\min }, 0\right\}, \min \left\{\gamma+z^{\max }, 1\right\}\right] .
$$

The cases in which no mixing is needed $(\gamma=0$ and $\gamma=1)$ can be realized exactly.

In the deterministic case, every solution $(\gamma, \beta)$ is realized exactly. In in case of decision uncertainty, however, there are many possible outcomes of the solution $(\gamma, \beta)$, one for each realization $\gamma+z$ with $z \in Z(\gamma)$. The plant nursery would like to avoid realizations in intervals that lead to low quality. That is, we only consider solutions $\gamma$, for which the plant can be sold in every realization. This is required in the next definition.

Definition 8. A feasible solution $(\gamma, \beta) \in \mathfrak{F}$ is called decision robust feasible if $Q(\gamma+z, \beta) \geq \alpha$ for all $z \in Z(\gamma)$.

The set of all decision robust feasible solutions is subsequently denoted as

$$
\mathcal{X}^{\mathrm{RC}}=\left\{(\gamma, \beta) \in[0,1] \times\{0,1\} \mid \gamma \leq v^{c o m p}, Q(\gamma+z, \beta) \geq \alpha \forall z \in Z(\gamma)\right\} .
$$

For every solution $\gamma$, we consider the set of its realizations and hence, in the objective space, the outcome of its realizations is a set rather than a point. As an optimality concept, we use the definition of decision robust efficiency by [10, Def.3] in an equivalent reformulation (given in [10, Eq.(3)]).

Definition 9. An element $\left(\gamma^{*}, \beta^{*}\right) \in \mathcal{X}^{\mathrm{RC}}$ is called a decision robust [weakly/./strictly] efficient solution if there is no $(\gamma, \beta) \in \mathcal{X}^{\mathrm{RC}} \backslash\left\{\left(\gamma^{*}, \beta^{*}\right)\right\}$ with the property

$$
\forall z \in Z(\gamma) \exists z^{\prime} \in Z\left(\gamma^{*}\right):\left(\begin{array}{c}
a d C(\gamma+z, \beta) \\
G W P(\gamma+z, \beta)
\end{array}\right)[</ \leq / \leqq]\left(\begin{array}{c}
a d C\left(\gamma^{*}+z^{\prime}, \beta^{*}\right) \\
G W P\left(\gamma^{*}+z^{\prime}, \beta^{*}\right)
\end{array}\right) .
$$

Note that, in contrast to the deterministic case, in the robust case the weakly and the strictly robust efficient solutions to the peat-and-pots problem do not coincide. In the remainder, the sets of decision robust [weakly/. / strictly] efficient solutions are denoted as $[w \mathcal{E} / \mathcal{E} / s \mathcal{E}]$ and the decision robust [weakly/. / strictly] efficient solutions for the fixed pot variable $\beta \in\{0,1\}$ are denoted as $[w \mathcal{E}(\beta) / \mathcal{E}(\beta) / s \mathcal{E}(\beta)]$, respectively. Note that we have by Definition 9

$$
s \mathcal{E} \subseteq \mathcal{E} \subseteq w \mathcal{E} \text { and } \quad s \mathcal{E}(\beta) \subseteq \mathcal{E}(\beta) \subseteq w \mathcal{E}(\beta)
$$

The sets $w \mathcal{E}$ and $s \mathcal{E}$ are also used to determine the set $\mathcal{E}$ in the next section.

\subsection{Decision robust efficient solutions to the peat-and-pots problem}

In order to determine the decision robust [weakly/ • / strictly] efficient solutions to the peatand-pots problem with decision uncertainty we again split the problem into two and solve it for $\beta=0$ (plastic pot) and for $\beta=1$ (biodegradable pot). The set of decision robust [weakly $/ \cdot$ /strictly] efficient solutions can then be derived by a pairwise comparison of the robust [weakly $/ \cdot /$ strictly] efficient sets of the two values of $\beta$. 
In the remainder of this section, we therefore assume that the pot variable $\beta \in\{0,1\}$ is fixed and we determine the decision robust [weakly $/ \cdot /$ strictly] efficient solutions with respect to each $\beta$. Applying Definition 8, we define the decision robust feasible set for the fixed pot variable $\beta \in\{0,1\}$ as

$$
\mathcal{X}_{\beta}^{\mathrm{RC}}:=\left\{\gamma \mid(\gamma, \beta) \in \mathcal{X}^{\mathrm{RC}}\right\} .
$$

A specific property of the solutions of the peat-and-pots problem with decision uncertainty is presented in Proposition 10: For all $\gamma \in \mathcal{X}_{\beta}^{\mathrm{RC}}$, with $\gamma>\left|z^{m i n}\right|$, there is no need to distinguish between decision robust weakly and strictly efficiency.

Proposition 10. Let $\beta \in\{0,1\}$ be fixed and let $\bar{\gamma} \in \mathcal{X}_{\beta}^{\mathrm{RC}}$ be a solution with $\left|z^{\text {min }}\right|<\bar{\gamma}$. Then the solution $\bar{\gamma}$ is decision robust weakly efficient for the peat and pots problem with decision uncertainty if and only if it is decision robust efficient and if and only if it is decision robust strictly efficient.

Proof. By Definition 8, every decision robust strictly efficient solution is also decision robust efficient and decision robust weakly efficient.

In order to show the reverse direction, we assume that $\bar{\gamma} \in \mathcal{X}_{\beta}^{\mathrm{RC}}$ with $\left|z^{\text {min }}\right|<\bar{\gamma}$ is not decision robust strictly efficient and we show that it is not decision robust weakly efficient. Because $\bar{\gamma}$ is not decision robust strictly efficient, there exists $\gamma^{\dagger} \in \mathcal{X}_{\beta}^{\mathrm{RC}} \backslash\{\bar{\gamma}\}$ such that for every $z^{\dagger} \in Z\left(\gamma^{\dagger}\right)$ there exists $\bar{z} \in Z(\bar{\gamma})$ such that

$$
a d C\left(\gamma^{\dagger}+z^{\dagger}, \beta\right) \leq a d C(\bar{\gamma}+\bar{z}, \beta) \quad \text { and } \quad G W P\left(\gamma^{\dagger}+z^{\dagger}, \beta\right) \leq G W P(\bar{\gamma}+\bar{z}, \beta) .
$$

By assumption, we have $0 \leq-z^{\text {min }}<\bar{\gamma}$ and, using (6), we obtain

$$
z^{\min }(\bar{\gamma})=z^{\min }
$$

We distinguish the two cases $\gamma^{\dagger} \in[0, \bar{\gamma}) \cap \mathcal{X}_{\beta}^{\mathrm{RC}}$ and $\gamma^{\dagger} \in(\bar{\gamma}, 1] \cap \mathcal{X}_{\beta}^{\mathrm{RC}}$.

In the first case, $\gamma^{\dagger} \in[0, \bar{\gamma}) \cap \mathcal{X}_{\beta}^{\mathrm{RC}}$, we use (8) to obtain $\gamma^{\dagger}+z^{\text {min }}\left(\gamma^{\dagger}\right)<\bar{\gamma}+z^{\text {min }} \leq \bar{\gamma}+\bar{z}$ for all $\bar{z} \in Z(\bar{\gamma})$, and, by Lemma 3 , it holds

$$
G W P(\bar{\gamma}+\bar{z}, \beta)<G W P\left(\gamma^{\dagger}+z^{\min }\left(\gamma^{\dagger}\right), \beta\right)
$$

for all $\bar{z} \in Z(\bar{\gamma})$ in contradiction to (10).

In the second case, $\gamma^{\dagger} \in(\bar{\gamma}, 1]$, we consider an arbitrary element $z^{\dagger} \in Z\left(\gamma^{\dagger}\right)$. By (10), there exists $\bar{z} \in Z(\bar{\gamma})$ such that $a d C\left(\gamma^{\dagger}+z^{\dagger}, \beta\right) \leq a d C(\bar{\gamma}+\bar{z}, \beta)$ and $G W P\left(\gamma^{\dagger}+z^{\dagger}, \beta\right) \leq$ $G W P(\bar{\gamma}+\bar{z}, \beta)$. We distinguish the two cases $\gamma^{\dagger}+z^{\dagger} \neq \bar{\gamma}+\bar{z}$ and $\gamma^{\dagger}+z^{\dagger}=\bar{\gamma}+\bar{z}$.

In Case 2.1, $\gamma^{\dagger}+z^{\dagger} \neq \bar{\gamma}+\bar{z}$, using Proposition 5, we directly obtain $\operatorname{adC}\left(\gamma^{\dagger}+z^{\dagger}, \beta\right)<$ $a d C(\bar{\gamma}+\bar{z}, \beta)$ and $G W P\left(\gamma^{\dagger}+z^{\dagger}, \beta\right)<G W P(\bar{\gamma}+\bar{z}, \beta)$.

In Case 2.2, $\gamma^{\dagger}+z^{\dagger}=\bar{\gamma}+\bar{z}$, we have $0 \leq-z^{\text {min }}<\bar{\gamma}<\gamma^{\dagger}$ by assumption and, by (8), we obtain

$$
0<\bar{\gamma}+z^{m i n}<\gamma^{\dagger}+z^{m i n} \leq \gamma^{\dagger}+z^{\dagger}=\bar{\gamma}+\bar{z} .
$$

Moreover, there exists $i \in\{1, \ldots, \omega\}$ such that $\gamma^{\dagger}+z^{\dagger} \in\left(t_{i-1}^{\beta}, t_{i}^{\beta}\right]$ and hence, we have

$$
\gamma^{\dagger}+z^{\dagger} \in\left(\bar{\gamma}+z^{m i n}, \bar{\gamma}+\bar{z}\right] \cap\left(t_{i-1}^{\beta}, t_{i}^{\beta}\right] .
$$

Therefore, there exists $z^{\prime} \in\left[z^{\text {min }}, \bar{z}\right] \subseteq Z(\bar{\gamma})$ such that $\bar{\gamma}+z^{\prime} \in\left(\bar{\gamma}+z^{\text {min }}, \bar{\gamma}+\bar{z}\right) \cap\left(t_{i-1}^{\beta}, t_{i}^{\beta}\right]$. We then have $\bar{\gamma}+z^{\prime}<\gamma^{\dagger}+z^{\dagger}$ and $Q\left(\bar{\gamma}+z^{\prime}, \beta\right) \leq Q\left(\gamma^{\dagger}+z^{\dagger}, \beta\right)$ by definition of $Q$. Applying 
Lemma 1 , we obtain $a d C\left(\gamma^{\dagger}+z^{\dagger}, \beta\right)<a d C\left(\bar{\gamma}+z^{\prime}, \beta\right)$ and $G W P\left(\gamma^{\dagger}+z^{\dagger}, \beta\right)<G W P\left(\bar{\gamma}+z^{\prime}, \beta\right)$. This then implies that $\bar{\gamma}$ is not decision robust weakly efficient according to Definition 8 in contradiction to our assumption.

We next present an analytical description of the set of decision robust [weakly/· / strictly] efficient solutions. To this end, a candidate set is constructed. We first define the set of shifted interval bounds

$$
R(\beta):=\left\{t_{i}^{\beta}-z^{\max } \mid 1 \leq i \leq \omega\right\} \cup\left\{0, \min \left\{v^{\text {comp }}, 1\right\}\right\}
$$

and we restrict this set to its decision robust feasible elements and we define

$$
\begin{aligned}
S(\beta) & :=R(\beta) \cap \mathcal{X}_{\beta}^{\mathrm{RC}} \\
& =\left\{s \in R(\beta) \mid 0 \leq s \leq v^{\text {comp }}, \alpha \leq \min _{z \in Z(s)} Q(s+z, \beta)\right\},
\end{aligned}
$$

where the second equality follows from (9). We also use the notation

$$
\delta_{\max }^{\beta}:=\max _{s \in S(\beta)} s \text { and } j_{\max }^{\beta}:=\min _{z \in Z\left(\delta_{\max }^{\beta}\right)} Q\left(\delta_{\max }^{\beta}+z, \beta\right) .
$$

In order to determine the set of decision robust [weakly/./strictly] solutions, we then define the candidate set

$$
\mathcal{W}(\beta):=\bigcup_{j=j_{\max }^{\beta}}^{\nu}\left\{\max \left\{s \in S(\beta) \mid j \leq \min _{z \in Z(s)} Q(s+z, \beta)\right\}\right\} .
$$

Note that $\mathcal{W}(\beta) \subseteq S(\beta)$ by definition and hence, $\mathcal{W}(\beta)$ is a finite set that can easily be calculated by comparing the elements of the finite set $S(\beta)$ with respect to the minimum quality in their realization sets.

The choice of $\mathcal{W}(\beta)$ as the candidate set is next motivated by showing that each solution is dominated by one solution of $\mathcal{W}(\beta)$ with respect to the minimum quality of the realization set.

Lemma 11. For every $\gamma \in \mathcal{X}_{\beta}^{\mathrm{RC}}$, there exists $\delta \in \mathcal{W}(\beta)$ such that $\gamma \leq \delta$ and

$$
\min _{z \in Z(\gamma)} Q(\gamma+z, \beta) \leq \min _{z \in Z(\delta)} Q(\delta+z, \beta) .
$$

Proof. It is sufficient to show the claim for $\gamma \in \mathcal{X}_{\beta}^{\mathrm{RC}}$ with $0 \neq \gamma$, since $0 \in S(\beta)$. For every $\gamma \in \mathcal{X}_{\beta}^{\mathrm{RC}} \backslash\{0\}$, there exists $i \in\{1, \ldots, \omega\}$ such that $\gamma+z^{\max } \in\left(t_{i-1}^{\beta}, t_{i}^{\beta}\right]$, and we define

$$
d:=\left\{\begin{array}{ll}
\min \left\{t_{i}^{\beta}-z^{\max }, v^{\operatorname{comp}}\right\} & \text { if } \gamma<1-z^{\max } \\
\min \left\{1, v^{\operatorname{comp}}\right\}, & \text { if } \gamma \geq 1-z^{\max }
\end{array} .\right.
$$

We then have $0<\gamma \leq d$, and by (8), we have $\{d\}+Z(d) \subseteq(\{\gamma\}+Z(\gamma)) \cup\left(t_{i-1}^{\beta}, t_{i}^{\beta}\right]$, and we hence obtain

$$
\alpha \leq \min _{z \in Z(\gamma)} Q(\gamma+z, \beta) \leq \min _{z \in Z(d)} Q(d+z, \beta) .
$$


This implies $d \in \mathcal{X}_{\beta}^{\mathrm{RC}}$ and hence, we have $d \in S(\beta)$. Denoting $j:=\min _{z \in Z(\gamma)} Q(\gamma+z, \beta)$, we directly obtain

$$
\gamma \leq d \leq \max \left\{s \in S(\beta) \mid j \leq \min _{z \in Z(s)} Q(s+z, \beta)\right\} \leq \delta_{\max }^{\beta}
$$

We then define

$$
\delta:= \begin{cases}\max \left\{s \in S(\beta) \mid j \leq \min _{z \in Z(s)} Q(s+z, \beta)\right\}, & \text { if } j_{\text {max }}^{\beta}<j \\ \delta_{\max }^{\beta}, & \text { if } j \leq j_{\text {max }}^{\beta}\end{cases}
$$

and we obtain $\delta \in \mathcal{W}(\beta)$, and $\gamma \leq d \leq \delta$.

The next result relates the set $\mathcal{W}(\beta)$ to the sets of decision robust weakly and strictly efficient solutions. The sets of decision robust weakly and strictly efficient solutions bound the set of decision robust efficient solutions, i.e., $s \mathcal{E}(\beta) \subseteq \mathcal{E}(\beta) \subseteq w \mathcal{E}(\beta)$, and, as shown next, these also bound the candidate set, i.e., $s \mathcal{E}(\beta) \subseteq \mathcal{W}(\beta) \subseteq w \mathcal{E}(\beta)$.

Proposition 12. The following are true.

a) Every element of the set $\mathcal{W}(\beta)$ is decision robust weakly efficient, i.e., $\mathcal{W}(\beta) \subseteq w \mathcal{E}(\beta)$.

b) Every strictly robust efficient solution is an element of $\mathcal{W}(\beta)$, i.e., $s \mathcal{E}(\beta) \subseteq \mathcal{W}(\beta)$.

Proof. We first show that all elements of $\mathcal{W}(\beta)$ are decision robust weakly efficient. We consider $\bar{\gamma} \in \mathcal{W}(\beta)$ and we show that for every decision robust feasible solution $\gamma^{\dagger} \in \mathcal{X}_{\beta}^{\mathrm{RC}} \backslash\{\bar{\gamma}\}$ there exists $z^{\dagger} \in Z\left(\gamma^{\dagger}\right)$ such that for all $z \in Z(\bar{\gamma})$ it holds $a d C(\bar{\gamma}+z, \beta) \leq a d C\left(\gamma^{\dagger}+z^{\dagger}, \beta\right)$ or $G W P\left(\bar{\gamma}+z^{\dagger}, \beta\right) \leq G W P\left(\gamma^{\dagger}+z, \beta\right)$.

We consider an arbitrary $\gamma^{\dagger} \in \mathcal{X}_{\beta}^{\mathrm{RC}} \backslash\{\bar{\gamma}\}$ and we distinguish the two cases $\gamma^{\dagger} \in[0, \bar{\gamma}) \cap \mathcal{X}_{\beta}^{\mathrm{RC}}$ and $\gamma^{\dagger} \in(\bar{\gamma}, 1] \cap \mathcal{X}_{\beta}^{\mathrm{RC}}$.

In the first case, $\gamma^{\dagger} \in[0, \bar{\gamma}) \cap \mathcal{X}_{\beta}^{\mathrm{RC}}$, we have $0 \leq \gamma^{\dagger}+z^{\text {min }}\left(\gamma^{\dagger}\right) \leq \bar{\gamma}+z$ for all $z \in Z(\bar{\gamma})$ by (8). According to Lemma 3, we then obtain $G W P(\bar{\gamma}+z, \beta) \leq G W P\left(\gamma^{\dagger}+z^{\min }\left(\gamma^{\dagger}\right), \beta\right)$ for all $z \in Z(\bar{\gamma})$.

In the second case, $\gamma^{\dagger} \in(\bar{\gamma}, 1] \cap \mathcal{X}_{\beta}^{\mathrm{RC}}$, we have $\bar{\gamma}<\gamma^{\dagger}$ and we consider

$$
z^{\dagger} \in \underset{z \in Z\left(\gamma^{\dagger}\right)}{\arg \min } Q\left(\gamma^{\dagger}+z, \beta\right)
$$

By Lemma 11, there exists $\delta^{\dagger} \in \mathcal{W}(\beta)$ such that $\gamma^{\dagger} \leq \delta^{\dagger}$ and

$$
Q\left(\gamma^{\dagger}+z^{\dagger}, \beta\right) \leq \min _{z \in Z\left(\delta^{\dagger}\right)} Q\left(\delta^{\dagger}+z, \beta\right)<\min _{z \in Z(\bar{\gamma})} Q(\bar{\gamma}+z, \beta)
$$

where the last inequality follows from the definition of the set $\mathcal{W}(\beta)$. According to Lemma 2 , we then have $a d C(\bar{\gamma}+z, \beta)<a d C\left(\gamma^{\dagger}+z^{\dagger}, \beta\right)$ for all $z \in Z(\bar{\gamma})$.

It remains to show that every solution in $\mathcal{X}_{\beta}^{\mathrm{RC}} \backslash \mathcal{W}(\beta)$ is not decision robust strictly efficient in the sense of Definition 8. For every $\gamma^{\prime} \in \mathcal{X}_{\beta}^{\mathrm{RC}} \backslash \mathcal{W}(\beta)$ we consider $j:=\min _{z \in Z\left(\gamma^{\prime}\right)} Q\left(\gamma^{\prime}+z, \beta\right)$ and by Lemma 11, the solution

$$
\left.\delta:=\max \left\{s \in \mathcal{W}(\beta) \mid j \leq \min _{z \in Z(s)} Q(s+z, \beta)\right\}\right\},
$$


satisfies $\gamma^{\prime}<\delta$.

We show that for every $\tilde{z} \in Z(\delta)$, there exists $z^{\prime} \in Z\left(\gamma^{\prime}\right)$ with the property $\operatorname{ad} C(\delta+\tilde{z}, \beta) \leq$ $a d C\left(\gamma^{\prime}+z^{\prime}, \beta\right)$ and $G W P(\delta+\tilde{z}, \beta) \leq G W P\left(\gamma^{\prime}+z^{\prime}, \beta\right)$.

By (8), we have $\{\delta\}+Z(\delta) \subseteq\left[\gamma^{\prime}+z^{\min }\left(\gamma^{\prime}\right), \delta+z^{\max }(\delta)\right]$ and for every element $\tilde{z} \in Z(\delta)$ we distinguish the two cases $\delta+\tilde{z} \in\left\{\gamma^{\prime}\right\}+Z\left(\gamma^{\prime}\right)$ and $\delta+\tilde{z} \in\left(\gamma^{\prime}+z^{\max }\left(\gamma^{\prime}\right), \delta+z^{\max }(\delta)\right]$.

In the first case, $\delta+\tilde{z} \in\left\{\gamma^{\prime}\right\}+Z\left(\gamma^{\prime}\right)$, there exists $z^{\prime} \in Z\left(\gamma^{\prime}\right)$ such that $\delta+\tilde{z}=\gamma^{\prime}+z^{\prime}$. Consequently, we have $\operatorname{adC}(\delta+\tilde{z}, \beta) \leq a d C\left(\gamma^{\prime}+z^{\prime}, \beta\right)$ and $G W P(\delta+\tilde{z}, \beta) \leq G W P\left(\gamma^{\prime}+z^{\prime}, \beta\right)$.

In the second case, $\delta+\tilde{z} \in\left(\gamma^{\prime}+z^{\max }\left(\gamma^{\prime}\right), \delta+z^{\max }(\delta)\right]$, we consider $z^{\prime} \in \arg \min \left\{Q\left(\gamma^{\prime}+z, \beta\right) \mid\right.$ $\left.z \in Z\left(\gamma^{\prime}\right)\right\}$. By the choice of $\delta$, we have $Q\left(\gamma^{\prime}+z^{\prime}, \beta\right) \leq Q(\delta+\tilde{z}, \beta)$ and, applying Lemma 1, we obtain $\operatorname{adC}(\delta+\tilde{z}, \beta)<a d C\left(\gamma^{\prime}+z^{\prime}, \beta\right)$ and $G W P(\delta+\tilde{z}, \beta)<G W P\left(\gamma^{\prime}+z^{\prime}, \beta\right)$.

The set of decision robust efficient solutions can be determined through the set $\mathcal{W}(\beta)$ as the next two main results show. If the sets of decision robust weakly and strictly efficient solutions coincide, cf. Proposition 10, then $\mathcal{W}(\beta)$ is the set of robust efficient solutions, see Theorem 13. However, if these sets do not coincide, they can be determined by Theorem 14 .

In Theorem 13, the set of decision robust [weakly/ • / strictly] efficient solutions are determined for the case that all candidates in $\mathcal{W}(\beta)$ are bigger than $\left|z^{m i n}\right|$. The results of Proposition 10 and Proposition 12 are combined to prove that $\mathcal{W}(\beta)$ is the set of decision robust efficient solutions in this case.

Theorem 13. If $\mathcal{W}(\beta)$ satisfies $\mathcal{W}(\beta) \cap\left[0,\left|z^{\text {min }}\right|\right]=\emptyset$, then the set

$$
\mathcal{E}(\beta)=s \mathcal{E}(\beta)=w \mathcal{E}(\beta)=\mathcal{W}(\beta)
$$

is the set of decision robust [weakly/·/strictly] efficient solutions.

Proof. We first show that every solution $\gamma^{\prime} \in\left[0,\left|z^{m i n}\right|\right]$ is not decision robust weakly efficient. By assumption, we have $0 \notin \mathcal{W}(\beta)$ and, for $j^{0}:=Q(0, \beta)$, there exists $d \in \mathcal{W}(\beta)$ such that $0<d$ and $j^{0} \leq Q(d+z, \beta)$ for all $z \in Z(d)$ according to Lemma 11. By (8), we then have $\gamma^{\prime}+z^{\min }\left(\gamma^{\prime}\right)=0<d+z$ and

$$
Q\left(\gamma^{\prime}+z^{\min }\left(\gamma^{\prime}\right), \beta\right)=j^{0} \leq Q(d+z, \beta)
$$

for all $z \in Z(d)$. Applying Lemma 1, we obtain that for every $z \in Z(d)$, there is $z^{\min }\left(\gamma^{\prime}\right) \in$ $Z\left(\gamma^{\prime}\right)$ such that $a d C(d+z, \beta)<a d C\left(\gamma^{\prime}+z^{\min }\left(\gamma^{\prime}\right), \beta\right)$ and $G W P(d+z, \beta)<G W P\left(\gamma^{\prime}+\right.$ $\left.z^{\min }\left(\gamma^{\prime}\right), \beta\right)$ and hence, the solution $\gamma^{\prime}$ is not decision robust weakly efficient. As a consequence, the set of decision robust weakly efficient efficient solutions is a subset of $\left(-z^{\min }, 1\right] \cap$ $\mathcal{X}_{\beta}^{\mathrm{RC}}$ and, by Proposition 10, the decision robust weakly efficient solutions and the decision robust strictly efficient solutions coincide. The claim then follows directly from Proposition 12 .

The factor that determines whether weakly and strictly decision robust efficiency coincide is a solution's closeness to 0 , i.e., the two coincide if a solution $\gamma$ satisfies $\gamma>\left|z^{\text {min }}\right|$ by Proposition 10. Note that, since less than $0 \%$ compost can never occur, the realization sets of the solutions smaller than $\left|z^{\text {min }}\right|$ are nested within in each other which makes their outcomes hard to distinguish, leading to weakly decision robust efficient solutions. This is shown in Theorem 14 .

Those elements of $\mathcal{W}(\beta)$ that are larger than $\left|z^{\text {min }}\right|$ are decision robust strictly efficient which can be shown by using Proposition 10, similarly to the proof of Theorem 13. However, since Proposition 10 only refers to solutions $\gamma>\left|z^{\text {min }}\right|$, the case of solutions smaller than $\left|z^{\text {min }}\right|$ has to be investigated seperately and different arguments have to be used. 
Theorem 14. If $\mathcal{W}(\beta)$ satisfies $\mathcal{W}(\beta) \cap\left[0,\left|z^{\text {min }}\right|\right] \neq \emptyset$, the following are true.

a) The set of decision robust [weakly/·] efficient solutions is given as

$$
\mathcal{E}(\beta)=w \mathcal{E}(\beta)=\left[0, \gamma_{\mathcal{W}}^{\beta}\right] \cup \mathcal{W}(\beta),
$$

where $\gamma_{\mathcal{W}}^{\beta}:=\max \left\{\gamma \mid \gamma \in \mathcal{W}(\beta) \cap\left[0,\left|z^{\min }\right|\right]\right\}$.

b) The set of decision robust strictly efficient solutions is given as

$$
s \mathcal{E}(\beta)=\mathcal{W}(\beta) \backslash\left(0,\left|z^{m i n}\right|\right] .
$$

Note that the solution 0 is a special case, since it can always be put into practice exactly as planned and, to prepare the proof of Theorem 14, we investigate the solution 0 seperately in the next lemma.

Lemma 15. If we have $0 \in \mathcal{W}(\beta)$ then 0 is decision robust strictly efficient.

Proof. Because $0 \in \mathcal{W}(\beta)$ by assumption, we have for $j:=Q(0, \beta)$

$$
0=\max \left\{\gamma \in \mathcal{X}_{\beta}^{\mathrm{RC}} \mid j \leq \min _{z \in Z(\gamma)} Q(\gamma+z, \beta)\right\}
$$

and hence, for every $\gamma^{\prime} \in \mathcal{X}_{\beta}^{\mathrm{RC}} \backslash\{0\}$, there exists $z^{\prime} \in Z\left(\gamma^{\prime}\right)$ such that $Q\left(\gamma^{\prime}+z^{\prime}, \beta\right)<j=$ $Q(0, \beta)=Q(0+z, \beta)$ for all $z \in Z(0)=\{0\}$. By Lemma 2 , we hence obtain $\operatorname{adC}(0+z, \beta)<$ $\operatorname{adC}\left(\gamma^{\prime}+z^{\prime}, \beta\right)$ for all $z \in Z(0)$, and 0 is decision robust strictly efficient.

The following lemma is also used in the proof of Theorem 14. The aim is to determine the decision robust efficient solutions within the set $\left[0,\left|z^{m i n}\right|\right]$ and the next lemma shows that the solutions in $\left(\gamma_{\mathcal{W}}^{\beta},\left|z^{\min }\right|\right]$ can be neglected.

Lemma 16. Let $\mathcal{W}(\beta) \cap\left[0,\left|z^{\min }\right|\right] \neq \emptyset$, and let $\gamma_{\mathcal{W}}^{\beta}:=\max \left\{\gamma \mid \gamma \in \mathcal{W}(\beta) \cap\left[0,\left|z^{\text {min }}\right|\right]\right\}$. If $\gamma_{\mathcal{W}}^{\beta}<\left|z^{m i n}\right|$, then the elements of the $\operatorname{set}\left(\gamma_{\mathcal{W}}^{\beta},\left|z^{m i n}\right|\right]$ are not decision robust weakly efficient.

Proof. By Lemma 11, for every $\gamma^{\prime} \in\left(\gamma_{\mathcal{W}}^{\beta},\left|z^{m i n}\right|\right]$, there exists $\delta \in \mathcal{W}(\beta)$ such that $\gamma^{\prime} \leq \delta$ and

$$
j:=\min _{z \in Z\left(\gamma^{\prime}\right)} Q\left(\gamma^{\prime}+z, \beta\right) \leq \min _{z \in Z(\delta)} Q(\delta+z, \beta)
$$

and by the assumption $\gamma^{\prime} \notin \mathcal{W}(\beta)$, we even have $0<\gamma^{\prime}<\delta$. We consider an arbitrary $\tilde{z} \in Z(\delta)$, and we show that there exists $z^{\prime} \in Z\left(\gamma^{\prime}\right)$ such that $\gamma^{\prime}+z^{\prime}<\delta+\tilde{z}$ and $Q\left(\gamma^{\prime}+z^{\prime}, \beta\right) \leq Q(\delta+\tilde{z}, \beta)$. Applying Lemma 1, we then obtain that the solution $\gamma^{\prime}$ is not decision robust weakly efficient.

Because $-z^{\min }<\delta$ by assumption, we have $0<\delta+\tilde{z}$ and there exists $i \in\{1, \ldots, \omega\}$ such that $\delta+\tilde{z} \in\left(t_{i-1}^{\beta}, t_{i}^{\beta}\right]$. We distinguish the two cases $\gamma^{\prime}+z^{\max } \leq t_{i-1}^{\beta}$ and $\gamma^{\prime}+z^{\text {max }}>t_{i-1}^{\beta}$.

In the first case, $\gamma^{\prime}+z^{\max } \leq t_{i-1}^{\beta}$, we consider any $z^{\prime} \in Z\left(\gamma^{\prime}\right)$ with $Q\left(\gamma^{\prime}+z^{\prime}, \beta\right)=j$ and we directly obtain $\gamma^{\prime}+z^{\prime} \leq \gamma^{\prime}+z^{\max } \leq t_{i-1}^{\beta}<\delta+\tilde{z}$ and $Q\left(\gamma^{\prime}+z^{\prime}, \beta\right)=j \leq Q(\delta+\tilde{z}, \beta)$.

In the second case, $t_{i-1}^{\beta}<\gamma^{\prime}+z^{\text {max }}$, we have $\left(t_{i-1}^{\beta}, \gamma^{\prime}+z^{\text {max }}\right) \subseteq\left[0, \gamma^{\prime}+z^{\text {max }}\right) \subseteq\left\{\gamma^{\prime}\right\}+Z\left(\gamma^{\prime}\right)$ and hence, there exists $z^{\prime} \in Z\left(\gamma^{\prime}\right)$ such that $t_{i-1}^{\beta}<\gamma^{\prime}+z^{\prime}<\delta+\tilde{z}$. Because of $\gamma^{\prime}+z^{\prime} \in$ $\left(t_{i-1}^{\beta}, \delta+\tilde{z}\right) \subseteq\left(t_{i-1}^{\beta}, t_{i}^{\beta}\right]$, we have $Q\left(\gamma^{\prime}+z^{\prime}, \beta\right)=Q(\delta+\tilde{z}, \beta)$ by the definition of the piecewise constant quality function $Q(\cdot, \beta)$. 
In the following lemma, it is shown that the remaining solutions in $\left[0,\left|z^{\text {min }}\right|\right]$, i.e., those that remain unconsidered in Lemma 16, are decision robust efficient.

Lemma 17. Let $\mathcal{W}(\beta) \cap\left[0,\left|z^{\text {min }}\right|\right] \neq \emptyset$, and let $\gamma_{\mathcal{W}}^{\beta}:=\max \left\{\gamma \mid \gamma \in \mathcal{W}(\beta) \cap\left[0, z^{\text {min }}\right]\right\}$. If $\gamma_{\mathcal{W}}^{\beta}<-z^{\text {min }}$, then the elements of the set $\left[0, \gamma_{\mathcal{W}}^{\beta}\right]$ are decision robust efficient.

Proof. We consider $\bar{\gamma} \in\left[0, \gamma_{\mathcal{W}}^{\beta}\right]$ and we show that $\bar{\gamma}$ is decision robust efficient. The solution $\bar{\gamma}$ satisfies $\{\bar{\gamma}\}+Z(\bar{\gamma}) \subseteq\left\{\gamma_{\mathcal{W}}^{\beta}\right\}+Z\left(\gamma_{\mathcal{W}}^{\beta}\right)$ by $(8)$, and we obtain $\bar{\gamma} \in \mathcal{X}_{\beta}^{\mathrm{RC}}$, since $\gamma_{\mathcal{W}}^{\beta} \in \mathcal{W}(\beta) \subseteq \mathcal{X}_{\beta}^{\mathrm{RC}}$. To show that $\bar{\gamma}$ is decision robust efficient, we consider any other $\gamma^{\dagger} \in \mathcal{X}_{\beta}^{\mathrm{RC}} \backslash\{\bar{\gamma}\}$ and we distinguish the two cases $\gamma^{\dagger} \in\left[0, \gamma_{\mathcal{W}}^{\beta}\right] \cap \mathcal{X}_{\beta}^{\mathrm{RC}}$ and $\gamma^{\dagger} \in\left(\gamma_{\mathcal{W}}^{\beta}, 1\right] \cap \mathcal{X}_{\beta}^{\mathrm{RC}}$.

In the first case, $\gamma^{\dagger} \in\left[0, \gamma_{\mathcal{W}}^{\beta}\right] \cap \mathcal{X}_{\beta}^{\mathrm{RC}}$, we have $\gamma^{\dagger} \leq \gamma_{\mathcal{W}}^{\beta} \leq-z^{\text {min }}$ and by (8) we have $\gamma^{\dagger}+z^{\min }\left(\gamma^{\dagger}\right)=0<\bar{\gamma}+z$ for all $z \in Z(\bar{\gamma}) \backslash\left\{z^{\min }(\bar{\gamma})\right\}$. Because the function GWP is monotonically decreasing, there is exactly one $\bar{z} \in Z(\bar{\gamma})$, i.e., $\tilde{z}:=z^{\min }(\bar{\gamma})$, such that $G W P\left(\gamma^{\dagger}+z^{\min }\left(\gamma^{\dagger}\right), \beta\right) \leq G W P(\bar{\gamma}+\tilde{z}, \beta)$ and then $\gamma^{\dagger}+z^{\min }\left(\gamma^{\dagger}\right)=0=\bar{\gamma}+\tilde{z}$.

In the second case, $\gamma^{\dagger} \in\left(\gamma_{\mathcal{W}}^{\beta}, 1\right] \cap \mathcal{X}_{\beta}^{\mathrm{RC}}$, we use again $\{\bar{\gamma}\}+Z(\bar{\gamma}) \subseteq\left\{\gamma_{\mathcal{W}}^{\beta}\right\}+Z\left(\gamma_{\mathcal{W}}^{\beta}\right)$ to obtain for every $\bar{z} \in Z(\bar{\gamma})$

$$
Q(\bar{\gamma}+\bar{z}, \beta) \geq \min _{z \in Z(\bar{\gamma})} Q(\bar{\gamma}+z, \beta) \geq \min _{z \in Z\left(\gamma_{\mathcal{W}}^{\beta}\right)} Q\left(\gamma_{\mathcal{W}}^{\beta}+z, \beta\right)>\min _{z \in Z\left(\gamma^{\dagger}\right)} Q\left(\gamma^{\dagger}+z, \beta\right),
$$

where the last inequality follows from $\gamma_{\mathcal{W}}^{\beta} \in \mathcal{W}(\beta)$ and Lemma 11. By Lemma 2 we obtain for $z^{\dagger} \in \arg \min _{z \in Z\left(\gamma^{\dagger}\right)} Q\left(\gamma^{\dagger}+z, \beta\right)$ that $a d C(\bar{\gamma}+z, \beta)<a d C\left(\gamma^{\dagger}+z^{\dagger}, \beta\right)$ for all $z \in Z(\bar{\gamma})$.

The results of the previous Lemma 15, Lemma 16 and Lemma 17 are next used to prove Theorem 14, i.e., to determine the set of decision robust [weakly/·/strictly] efficient solutions for the case that there is a candidate smaller than $\left|z^{\text {min }}\right|$.

Proof of Theorem 14. We first show a). We consider $\gamma \in \mathcal{X}_{\beta}^{\mathrm{RC}}$ and we distinguish the three cases $\gamma \in\left[0, \gamma_{\mathcal{W}}^{\beta}\right], \gamma \in\left(\gamma_{\mathcal{W}}^{\beta},\left|z^{\text {min }}\right|\right]$ and $\gamma \in\left(\left|z^{m i n}\right|, 1\right]$.

In the first case, $\gamma \in\left[0, \gamma_{\mathcal{W}}^{\beta}\right], \bar{\gamma}$ is decision robust efficient according to Lemma 17 . In the second case, $\gamma \in \mathcal{X}_{\beta}^{\mathrm{RC}} \cap\left(\gamma_{\mathcal{W}}^{\beta},\left|z^{m i n}\right|\right], \gamma$ is not decision robust weakly efficient according to Lemma 16 and hence, it is not decision robust efficient. In the third case, $\bar{\gamma} \in \mathcal{X}_{\beta}^{\mathrm{RC}} \cap\left(\left|z^{m i n}\right|, 1\right]$, Proposition 10 applies and every $\bar{\gamma} \in \mathcal{X}_{\beta}^{\mathrm{RC}} \cap\left(\left|z^{m i n}\right|, 1\right]$ is decision robust weakly efficient if and only if it is decision robust strictly efficient. By Proposition 12, we then obtain that $\bar{\gamma} \in \mathcal{X}_{\beta}^{\mathrm{RC}} \cap\left(\left|z^{m i n}\right|, 1\right]$ is decision robust [weakly/.] efficient if and only if $\bar{\gamma} \in \mathcal{W}(\beta)$.

We next show b). By a), every element of $\mathcal{W}(\beta) \backslash\left[0,\left|z^{\text {min }}\right|\right]$ is decision robust weakly efficient and it hence is decision robust strictly efficient according to Proposition 10. Moreover, by Proposition 12 and Lemma 15, the solution $0 \in \mathcal{X}_{\beta}^{\mathrm{RC}}$ is decision robust strictly efficient if and only if $0 \in \mathcal{W}(\beta)$.

It remains to show that any $\gamma^{\prime} \in\left(0,\left|z^{\text {min }}\right|\right]$ is not decision robust strictly efficient. Because $\gamma^{\prime} \leq-z^{\text {min }}$, we have $\gamma^{\prime}+z^{\min }\left(\gamma^{\prime}\right)=0$ by $(8)$, and hence, we have $\operatorname{adC}(0+z, \beta) \leq \operatorname{adC}\left(\gamma^{\prime}+\right.$ $\left.z^{\min }\left(\gamma^{\prime}\right), \beta\right)$ and $G W P(0+z, \beta) \leq G W P\left(\gamma^{\prime}+z^{\min }\left(\gamma^{\prime}\right), \beta\right)$ for all $z \in Z(0)=\{0\}$ and $\gamma^{\prime}$ is not decision robust strictly efficient by Definition 9 .

The results of this section can directly be used to determine the decision robust [weakly/. /strictly] efficient solutions to the peat-and-pots problem in two steps. In a first step, the candidate set $\mathcal{W}(\beta)$ can be calculated for each $\beta \in\{0,1\}$ from the small finite set that is 
the set of interval bounds of the quality function $Q$ minus the maximum perturbation $z^{\max }$. By Theorem 13 and Theorem 14, the candidates only have to be compared with respect to their size and their realization's minimum quality to determine the decision robust [weakly/. /strictly] efficient solutions. In a second step, the resulting sets can then be compared pairwise with respect to the two objective functions.

\section{Generalization of the results}

The presented solution approaches for the deterministic and the uncertain peat-and-pots problem can be generalized to a wider class of problems. There are various products in the market whose selling price depends on the quality of the products. Discrete quality levels are a natural approach to relate a product's quality and its price because they are easy to understand for customers. An option to decrease the environmental impacts of production processes is to replace the input matrials with eco-friendly alternatives. Therefore, a generalization of the solution approaches to the peat-and-pots problem is of interest for further applications that involve biobjective mixing problems where one objective function depends on the quality level of the final product and the costs of the input materials and where the second objective function is a measure for the environmental impact of the production process.

In order to generalize our results, we neglect the three specific properties inherent to the peat-and-pots problem that were introduced as three assumptions in Section 2. It can be assumed without loss of generality that the alternative input materials have a better GWP than the traditional materials, since this is part of the motivation for the problem, and hence, Assumption 3 can be neglected.

\subsection{Solutions in the absence of Assumption 1}

Products that are considered eco-friendly are often more expensive than the alternatives. Correspondingly, the case that the traditional input material of a production process is cheaper than its eco-friendly substitute has to be considered, which implies the opposite of Assumption 1. In this case, the first objective function is strictly increasing on every constant interval of the quality function, while the second objective function is strictly decreasing. The resulting sets of deterministic and decision robust efficient solutions are then finite unions of intervals rather than finite unions of points. For the deterministic solutions, this is due to a well-known classical result and a suitable result for decision robust solutions is proven in [10].

Algorithm 1 yields the set of [weakly/ / strictly] efficient solutions to the deterministic problem. The sets of [weakly $/ \cdot /$ strictly] efficient solutions coincide, because the two objective functions $a d C$ and GWP are injective if Assumption 1 does not hold while Assumption 2 holds. It is well-known in deterministic multiobjective optimization that an interval is efficient if the first objective function is strictly increasing and the second objective function is strictly decreasing. This also applies to the intervals of the quality function and such a constant interval is hence efficient if and only if one of its interior points is efficient. Algorithm 1 works as follows. Every quality level is considered, starting from the highest level and finishing with the level of the rightmost feasible solution. Because a higher quality level results in a higher selling price, all solutions in intervals with the highest quality level are efficient and they are first selected by the algorithm. Lower quality levels can only result in worse additional costs than the previously considered quality levels, but solutions larger than the previously selected solutions perform better in the second objective GWP. Therefore, for each quality level all 
intervals of this quality that lie on the right hand side of the intervals selected in the previous step of the algorithm are selected. The algorithm terminates after the quality level of the rightmost feasible solution $t_{\max }^{\beta}$ has been considered.

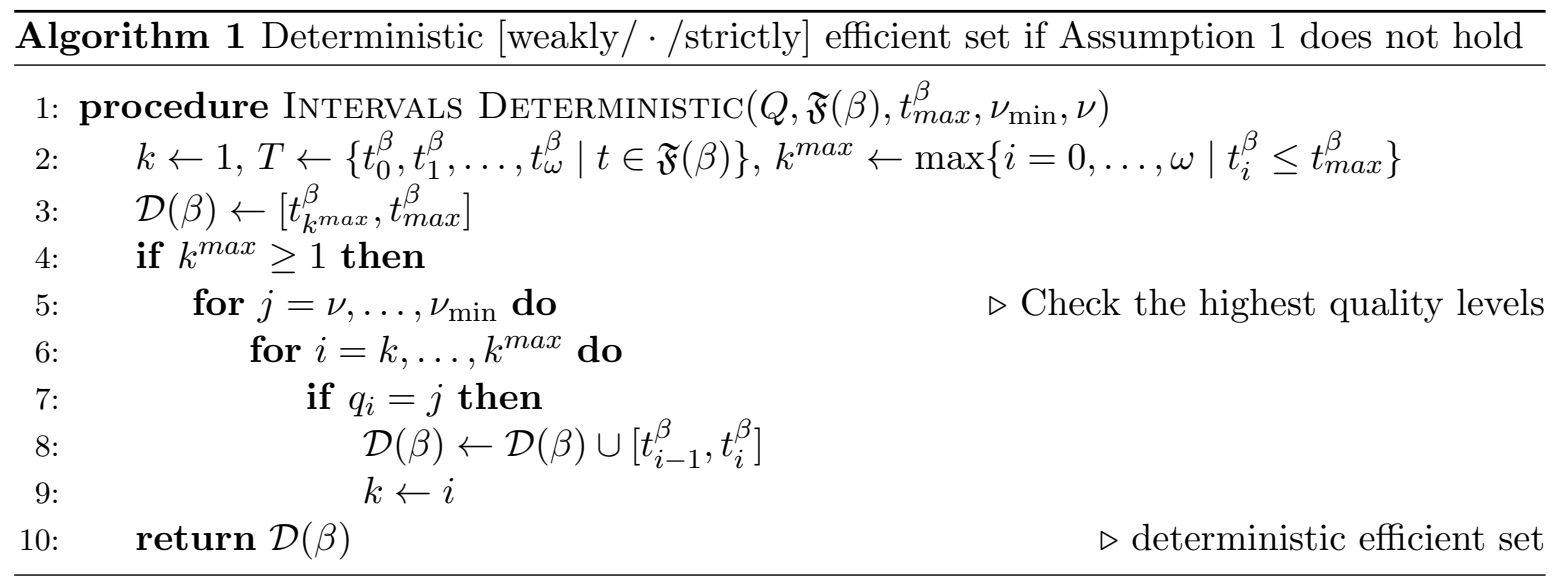

Algorithm 2 yields the set of decision robust [weakly/.] efficient solutions to the uncertain problem. The sets of decision robust [weakly/.] efficient solutions coincide because both objective functions are injective and hence, if a solution's outcome is strictly smaller than another solution's outcome with respect to one objective function, it is also strictly smaller with respect to the other objective function. The set of decision robust strictly efficient solutions generally does not coincide with this set.

In order to determine the set of decision robust [weakly/.] efficient solutions, a result on decision robustness for problems with at least one increasing and one decreasing objective function is used, see [10, Th. 27, Cor. 28], and it is adjusted to fit our problem. There are two differences between our problem and the problem addressed in the latter publication. First, in contrast to this work, the perturbation set $Z(\gamma)$ is considered as constant for all $\gamma \in \mathcal{X}_{\beta}^{\mathrm{RC}}$ and therefore, the cases where the set $Z(\gamma)$ differs from $\left[z^{\text {min }}, z^{\text {max }}\right]$, i.e., $\gamma \in$ $\left[0,\left|z^{\min }\right|\right] \cup\left[1-z^{\max }, 1\right]$, have to be considered as special cases. These special cases are covered in lines 6,18 and 20 of Algorithm 2 and it is noted that the case $\gamma \in\left(0,\left|z^{\text {min }}\right|\right)$ is covered by the general part of the algorithm in lines 8 to 17 . Second, the objective functions in that publication are assumed to be monotonic on the whole set of feasible solutions, while the objective functions in our problem are monotonic only on the constant intervals of the quality function. Due to these differences, the adjusted version of the result is that all solutions of a specific interval (see line 16 in Algorithm 2) are decision robust efficient if and only if the rightmost point of that interval is decision robust efficient. These specific intervals are related to the intervals of the quality function but a safety margin is added to obtain robust solutions and this safety margin corresponds to the maximum perturbation $\left|z^{\max }\right|$ on the rightmost point of each interval and the minimum perturbation $\left|z^{\text {min }}\right|$ at the leftmost point. Moreover, if two intervals of this kind overlap, the interval that is the union of the two is also entirely decision robust efficient, which is taken into account in line 12 of the algorithm. In summary, Algorithm 2 consists of a general case and three special cases of solutions that have different perturbation sets than the other solutions. 


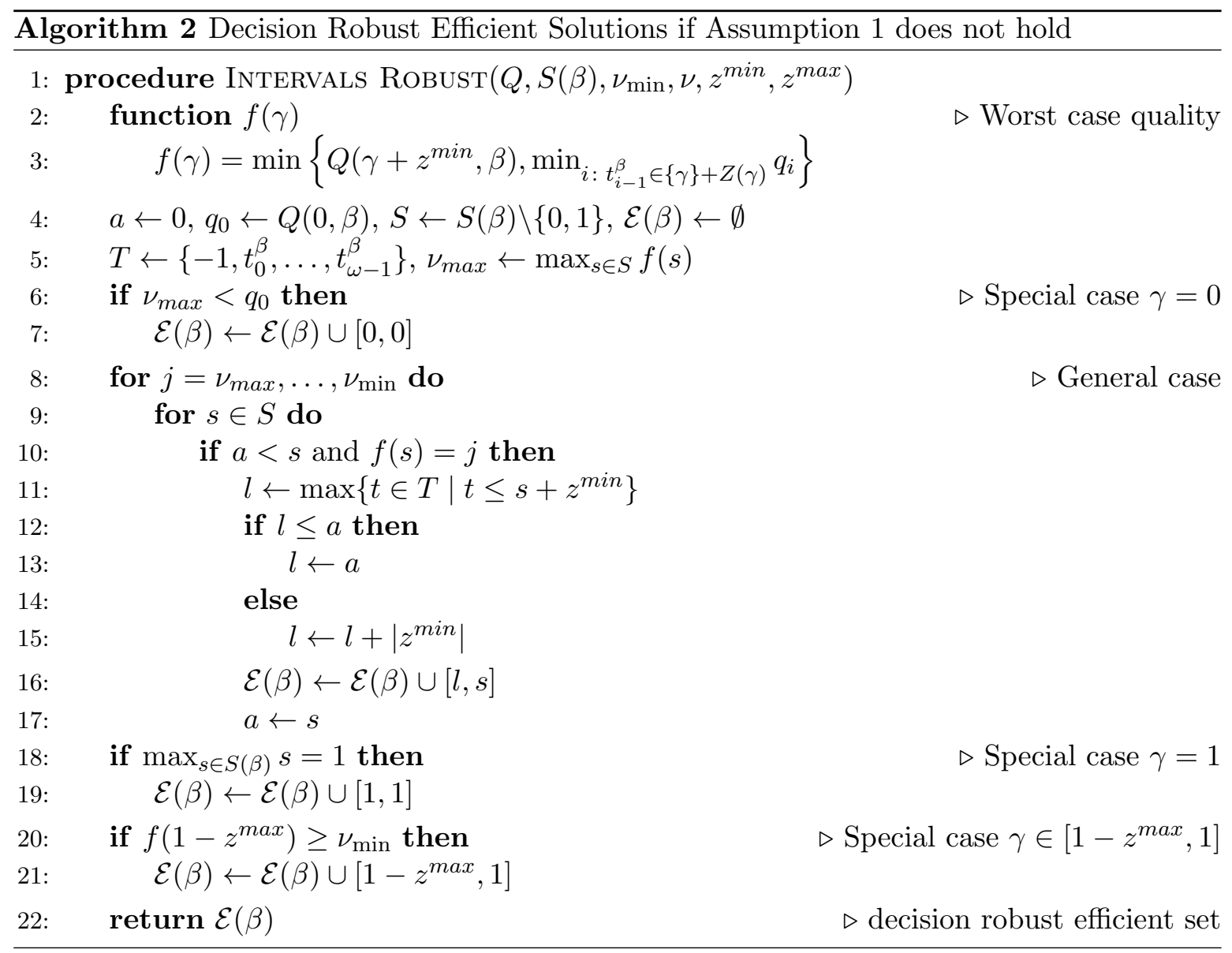




\subsection{Solutions in the absence of Assumption 2}

In the peat-and-pots problem, the first objective function is more affected by the selling price of the final product than the costs of the input matrials. Saving costs by chosing cheaper input matrials is then only sensible if the quality level can be maintained or raised. However, for other products the input materials are expensive enough to justify a reduction of costs at the expense of quality. In this case, Assumption 2 does not hold. As a consequence, solutions have to be compared directly by their additional costs rather than by their quality level. Furthermore, solutions can still be compared by their size since the objective function GWP is monotonically strictly decreasing.

Algorithm 3 yields the set of $[\cdot /$ strictly] efficient solutions to the deterministic problem. The sets of [-/strictly] efficient solutions coincide, because the second objective function GWP is strictly decreasing. The set of weakly efficient solutions generally does not coincide with this set. Because Assumption 1 still holds, the function $a d C$ is strictly decreasing on each of the constant intervals of the quality function and hence, only the right hand points of the intervals can be efficient solutions. The set of $[\cdot /$ strictly $]$ efficient solutions therefore is a finite union of points. Algorithm 3 works as follows. The rightmost feasible solution $t_{\max }^{\beta}$ is an efficient solution because it is the unique solution where GWP attains its minimum over the feasible set. This solution is selected at the start of the algorithm. Because only interval bounds can be efficient solutions, these are compared with respect to their outcome in $a d C$ and the maximum solution is chosen among those with a lower $a d C$-value than the solution selected in the previous step, see line 5 of the algorithm. Proceding in this way, every newly selected solution has a strictly better $a d C$-value and a stricly worse $G W P$-value than the previously selected solution and the resulting set is the set of efficient solutions.

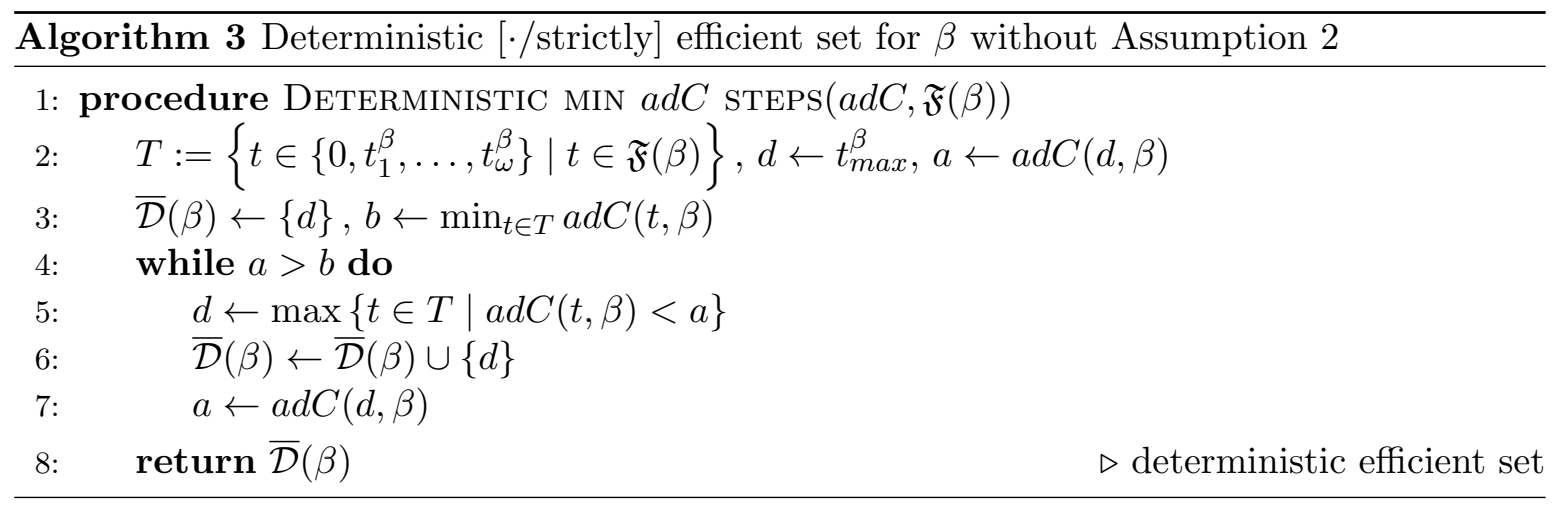

Algorithm 4 yields the set $\mathcal{V}(\beta)$ that can be used to determine the set of decision robust $[\cdot /$ strictly $]$ efficient solutions to the uncertain problem, by replacing the set $\mathcal{W}(\beta)$ in the results of Section 4.3. The sets of decision robust [-/strictly] efficient solutions coincide for solutions $\gamma>\left|z^{m i n}\right|$ because the objective function GWP is monotonically strictly decreasing. The set of decision robust weakly efficient solutions generally does not coincide with this set.

The main difference between the set $\mathcal{V}(\beta)$ and the previously used set $\mathcal{W}(\beta)$ is that a finite set of solutions is chosen with respect to their $a d C$-value rather than with respect to their quality level. The rightmost decision robust feasible solution $\delta_{\max }^{\beta}$ is always decision robust efficient because it is the best solution in terms of the maximum GWP-values of its realizations. Starting with this solution, Algorithm 4 proceeds similar to Algorithm 3 for the deterministic problem by selecting the rightmost candidate from a finite set that has a better 
$G W P$-value than the previously selected solution. However, there are two main differences between the two algorithms. First, the finite set of solutions $S(\beta)$ that is considered in Algorithm 4 corresponds to the interval bounds that are shifted by the safety margin $z^{\max }$ rather than the interval bounds themselves, see line 4 of the algorithm. Second, each candidate solution is evaluated and compared with respect to the supremum of $a d C$-values over the set of its realizations. The function that maps a solution to this supremum is defined in line 2 and solutions are selected according to their value with respect to this function in line 6 . The algorithm terminates when the minimum of that function over all decision robust feasible solutions has been attained.

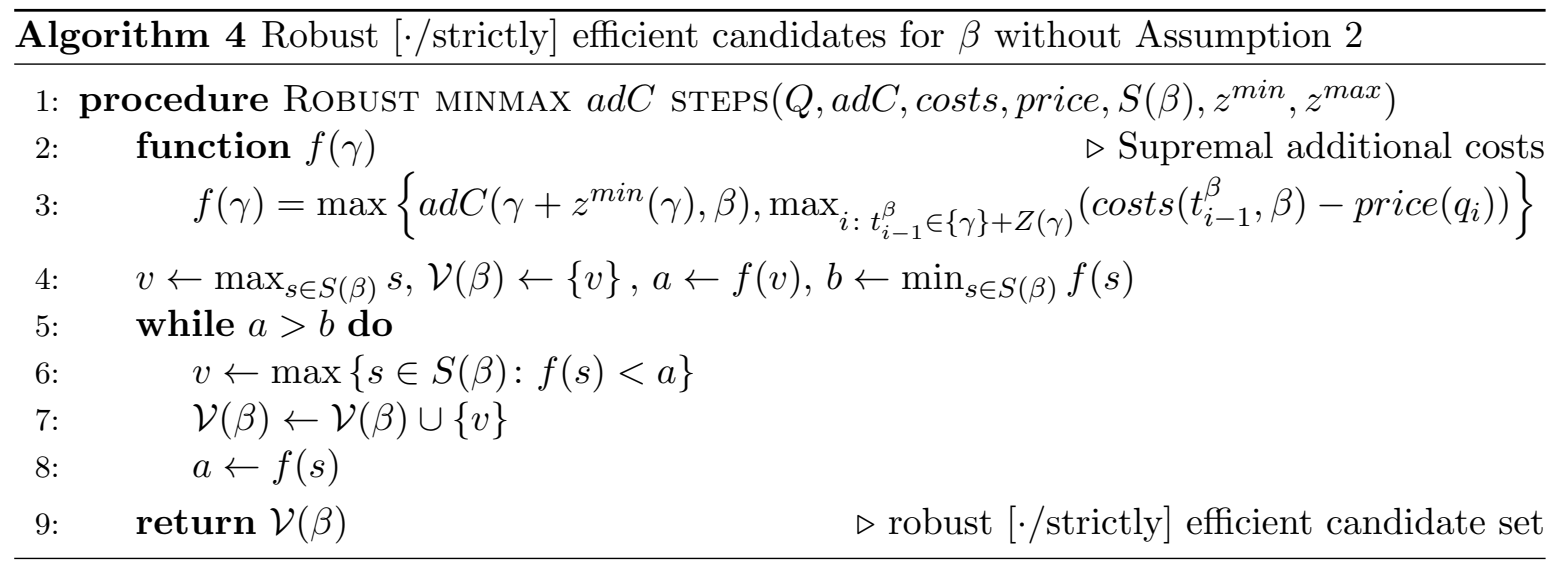

In order to determine the set of decision robust [-/strictly] efficient solutions, two cases are distinguished, as done in Section 4: the case that the sets of decision robust $[\cdot /$ strictly] efficient solutions coincide, and the case that the sets of decision robust [-/strictly] efficient solutions differ. For both cases, the sets of decision robust $[\cdot /$ strictly] efficient solutions are obtained by replacing the set $\mathcal{W}(\beta)$ by the set $\mathcal{V}(\beta)$ in Theorem 13 and in Theorem 14 .

\section{Summary and outlook}

The peat-and-pots problem is a bi-objective optimization problem with one linear objective function and one objective function that is the sum or a piecewise constant function and a linear function. The problem is modeled as a deterministic and as an uncertain problem. Solution approaches for both types of the problem are presented.

The classical solution concept for the deterministic problem is Pareto efficiency and an analytical description of the Pareto efficient solutions for each type of planter pot is given.

The uncertain peat-and-pots problem is addressed by means of robust multiobjective optimization. Two types of uncertainty, parameter and decision uncertainty, are taken into account and the uncertainty is addressed by the solution concepts robust efficiency and decision robust efficiency. For each type of planter pot, analytical descriptions of the decision robust efficient solutions are given or an algorithm to compute the set of solutions is developed.

The results of this work provide the theoretical background for applications of the considered problem such as the commercial cultivation of potted plants. The suggested solution approaches for both the deterministic and uncertain problem are applied to a case study on an Italian plant nursery in [18]. This work is particularly motivated by this practical problem and therefore, the peat-and-pots problem is solved in Section 3 and Section 4.3 for the specific 
case that is required by this application. According to the case study, the robust efficient solutions a preferable over the deterministic efficient solutions.

Because the deterministic efficient and the decision robust efficient solutions of the peatand-pots problem are both investigated in this work, the two sets of solutions can be compared. Under the assumptions that define the practical application in [18], each of the two solution sets can be computed from a finite union of points. The main difference between the deterministic and the robust solutions for each fixed pot $\beta$ is that the deterministic efficient solutions lie at the right hand sides of the constant intervals of the quality function, while there exists a safety margin between the robust solutions and the right hand sides of the intervals. However, a safety margin to the left hand interval bounds is not necessary. Moreover, as shown in this work, if any solution between 0 and the minimal perturbation $\left|z^{\text {min }}\right|$ is decision robust efficient then the whole interval between this point and zero is decision robust efficient. This is due to the structure of the perturbation sets $Z(\gamma)$, which are smaller for solutions lower than $\left|z^{\min }\right|$. The realization sets of the solutions near 0 are hence nested within each other and if one of them is decision robust efficient, the others are so as well. The latter property of the decision robust efficient solutions to the peat and pots problem therefore reflects the difference between decision uncertainty with constant perturbation sets as considered in [10] and perturbation sets that depend on the solution as considered here.

In Section 5, the results for the deterministic efficient and the decision robust efficient solutions from the previous sections are extended to the peat-and-pots problem in the absence of the three assumptions that reflect the setting of the practical application. Accordingly, the solutions to the deterministic and the uncertain problems can be computed by using the presented algorithms. Comparing the solution sets for the peat-and-pots problems with and without the assumptions, it is noted that the solution sets or candidate sets are finite unions of points if and only if the costs of the decision relevant traditional input materials are higher than the costs of the eco-friendly alternatives. Conversely, if the eco-friendly alternatives are more expensive than the traditional input materials, the solution sets and candidate sets are finite unions of compact intervals.

Further research includes applications of the presented results to different fields of industry. The results of this work could be extended to account for specific problem characteristics of further applications. For instance, a consumer's willingness to pay a higher price for ecofriendy products could be considered in the problem by adding a bonus to the monetary objective if the environmental impact of the solution is below a certain threshold. Also, the peat-and-pots problem could be modelled for two or more different species that require the same type of compost while the supply of compost is limited. Another option to extend the model is to consider additional objective functions, e.g., a second environmental objective function to simulateously assess different environmental aspects.

In summary, this work provides deterministic and robust solution approaches for the uncertain bi-objective optimization problem that is the peat-and-pots problem. Additionally, a generalized form of the problem is investigated and algorithms for the computation of the deterministic and the decision robust efficient solutions for these problems are given. Still, there are several options of further reasearch on extended versions of the problem that are relevant for various fields of application. 


\section{Acknowledgements}

This work was supported by DFG RTG 1703 "Resource Efficiency in Interorganizational Networks". The author would like to thank Anita Schöbel for her advice on the decision problem and the results presented here. The author would also like to thank Francesco Castellani and Jutta Geldermann for discussions on the case study that motivated this research.

\section{References}

[1] A. Ben-Tal and A. Nemirovski. Robust optimization - methodology and applications. Mathematical Programming, 92(3):453-480, 2002.

[2] A. Ben-Tal, L. El Ghaoui, and A. Nemirovski. Robust Optimization. Princeton University Press, Princeton and Oxford, 2009.

[3] D. Bertsimas, O. Nohadani, and K. M. Teo. Robust optimization in electromagnetic scattering problems. Journal of Applied Physics, 101(7):074507, 2007.

[4] C. Bohle, S. Maturana, and J. Vera. A robust optimization approach to wine grape harvesting scheduling. European Journal of Operational Research, 200(1):245-252, 2010.

[5] R. Bokrantz and A. Fredriksson. Necessary and sufficient conditions for pareto efficiency in robust multiobjective optimization. European Journal of Operational Research, 262 (2):682-692, 2017.

[6] V. Borodin, J. Bourtembourg, F. Hnaien, and N. Labadie. Handling uncertainty in agricultural supply chain management: A state of the art. European Journal of Operational Research, 254(2):348-359, 2016.

[7] M. Brandenburg, K. Govindan, J. Sarkis, and S. Seuring. Quantitative models for sustainable supply chain management: Developments and directions. European Journal of Operational Research, 233(2):299-312, 2014.

[8] G. J. Doole. Evaluation of an agricultural innovation in the presence of severe parametric uncertainty: An application of robust counterpart optimisation. Computers and Electronics in Agriculture, 84:16-25, 2012.

[9] M. Ehrgott, J. Ide, and A. Schöbel. Minmax robustness for multi-objective optimization problems. European Journal of Operational Research, 239:17-31, 2014. doi: 10.1016/j. ejor.2014.03.013.

[10] G. Eichfelder, C. Krüger, and A. Schöbel. Decision uncertainty in multiobjective optimization. Journal of Global Optimization, 69(2):485-510, 2017.

[11] J. Fliege and R. Werner. Robust multiobjective optimization \& applications in portfolio optimization. European Journal of Operational Research, 234(2):422 - 433, 2014.

[12] J. Geldermann, C. Jahn, T. Spengler, and O. Rentz. Proposal for an integrated approach for the assessment of cross-media aspects relevant for the determination of 'best available techniques' bat in the european union. The International Journal of Life Cycle Assessment, 4(2):94-106, 1999. 
[13] M. A. Goberna, V. Jeyakumar, G. Li, and J. Vicente-Pérez. Robust solutions of multiobjective linear semi-infinite programs under constraint data uncertainty. SIAM Journal on Optimization, 24(3):1402-1419, 2014.

[14] Jeroen C.J. Groot, Gerard J.M. Oomen, and Walter A.H. Rossing. Multi-objective optimization and design of farming systems. Agricultural Systems, pages 63-77, 2012.

[15] K. Hayashi. Multicriteria analysis for agricultural resource management: A critical survey and future perspectives. European Journal of Operational Research, 122(2):486-500, 2000 .

[16] L. E. Hombach, C. Büsing, and G. Walther. Robust and sustainable supply chains under market uncertainties and different risk attitudes - a case study of the german biodiesel market. European Journal of Operational Research, 2017.

[17] J. Ide and A. Schöbel. Robustness for uncertain multi-objective optimization: A survey and analysis of different concepts. OR Spectrum, 38(1):235-271, 2016.

[18] C. Krüger, F. Castellani, J. Geldermann, and A. Schöbel. Peat and pots: An application of robust multiobjective optimization to a mixing problem in agriculture. Technical Report 2018-4, Preprint-Reihe, Institut für Numerische und Angewandte Mathematik, Georg-August Universität Göttingen, 2018.

[19] D. Kuroiwa and G. M. Lee. On robust multiobjective optimization. Vietnam Journal of Mathematics, 40(2\&3):305-317, 2012.

[20] G. Lazzerini, S. Lucchetti, and F. P. Nicese. Analysis of greenhouse gas emissions from ornamental plant production: A nursery level approach. Urban Forestry \& Urban Greening, 13(3):517-525, 2014.

[21] D. E. Majewski, M. Wirtz, M. Lampe, and A. Bardow. Robust multi-objective optimization for sustainable design of distributed energy supply systems. Computers $\&$ Chemical Engineering, 102:26 - 39, 2017.

[22] M. Papafotiou, G. Kargas, and I. Lytra. Olive-mill waste compost as a growth medium component for foliage potted plants. HortScience, 40(6):1746-1750, 2005.

[23] M. Raviv. Composts in growing media: feedstocks, composting methods and potential applications. Acta Horticulturae, (1018):513-523, 2014.

[24] J. A. Robbins and M. R. Evans. Growing media for container production in a greenhouse or nursery. Cooperative Extension Service, University of Arkansas, US Department of Agriculture and county governments cooperating, 2001.

[25] E. Schettini, G. Santagata, M. Malinconico, B. Immirzi, G. Scarascia-Mugnozza, and G. Vox. Recycled wastes of tomato and hemp fibres for biodegradable pots: Physicochemical characterization and field performance. Resources, Conservation and Recycling, 70:9-19, 2013.

[26] J. M. Waddington, J. Plach, J. P. Cagampan, M. Lucchese, and M. Strack. Reducing the carbon footprint of canadian peat extraction and restoration. AMBIO: A Journal of the Human Environment, 38(4):194-200, 2009. 
D. Peat and pots: Analysis of Robust Solutions for a Biobjective Problem in Agriculture

[27] M. M. Wiecek and G. M. Dranichak. Robust Multiobjective Optimization for Decision Making Under Uncertainty and Conflict, chapter 4, pages 84-114. INFORMS, 2016. 


\section{Thank you all!}

First, I would like to thank my advisor Anita Schöbel for all her valuable advice and support during the last years and for all the answers I got when asking spontaneous questions through her open office door!

I would like to thank Gabriele Eichfelder for the inspiration during our cooperation and for her willingness to provide help when needed. I would also like to thank her for reading this thesis and for being in my thesis committee.

Next, I would like to thank my second advisor Russell Luke for helpful questions answers and comments during the time of my doctoral studies.

I would like to thank Margaret Wiecek for being a very motivating coauthor and for trying to improve my scientific English writing skills. I would also like to thank her for making me feel welcome during my research stay at Clemson University.

I thank my tandem partner and coauthor Francesco Castellani for all the communications about plant nurseries and global warming potential and for showing me new sorts of espresso. I would like to thank Jutta Geldermann for the interdisciplinary cooperation and the insights it provided into further areas of research. Moreover, I thank her and Carsten Mai for being my secondary advisors in the research training group "Resource Efficiency in Interorganizational Networks".

The financial support of the research training group is much appreciated, such as the opportunity to spend a research stay at Clemson University, South Carolina. I am also grateful for the friendly help in all organizational matters of the RTG by Kristin Golombek and Meike Schmehl.

The comprehensive help in all organizational or electronic matters the institute of numerical and applied mathematics by Antje Scholz, Petra Trapp, Jochen Schulz and Christoph Rügge is also highly valued, and so is the competent help of Carmen Barann concerning all questions about formal requirements of the Ph.D. programme.

A big thanks goes to the former and current colleagues of my working group: Alex, Anja, Emelie, Jonas I, Jonas H, Julius, Marc, Marco Be, Marco Bo, Marie, Mirko, Morten, Lisa, Philine, Sebastian, Sönke, Stephan and Thorsten! Thank you for a cheerful working atmosphere and breaks with coffee, cake, card games or chess and thank you for offering me a hand when needed! Special thanks goes to Philine for proofreading this thesis and to her, Alex and Emelie for supporting the writing of this thesis with lots of delicious homemade cookies!

Last but not least, I would like to thank my family and friends. There are so many ways in which you have been there for me, tirelessly offering help, support and whatever you think is needed. You are amazing! 



\section{Curriculum Vitae}

\section{Corinna Krüger}

\section{Persönliche Daten}

$\begin{array}{ll}\text { Wohnort } & \text { Göttingen } \\ \text { Geburtsort } & \text { Gifhorn } \\ \text { Staatsangehörigkeit } & \text { deutsch } \\ \text { E-Mail } & \text { ckrueger@math.uni-goettingen.de }\end{array}$

Akademische Ausbildung

15. April 2017

\section{Wissenschaftliche Mitarbeiterin}

bis 08. Oktober 2017 Universität Göttingen

15. April 2014

bis 14. April 2017

Seit April 2014

März 2014

Mai 2012

Juni 2007

\section{Wissenschaftliche Mitarbeiterin}

DFG Graduiertenkolleg 1703

"Ressourceneffizienz in Unternehmensnetzwerken"

Universität Göttingen

\section{Promotionsstudentin}

Institut für Numerische und Angewandte Mathematik Universität Göttingen

\section{M.Sc. Mathematik}

Universität Göttingen

B.Sc. Mathematik

Universität Göttingen

\section{Abitur}

Gymnasium Hankensbüttel 